

\section{MESENCHYMAL STEM CELLS AS \\ TROPHIC MEDIATORS IN CARTILAGE \\ REGENERATION}

Ling Wu 


\section{Committee}

Promotors: Prof. dr. M. Karperien University of Twente

Prof. dr. C.A. van Blitterswijk University of Twente

Chairman: Prof. dr. G. van der Steenhoven University of Twente

Members: $\quad$ Prof.. dr. F.P.J.G. Lafeber, UMC Utrecht

Dr. L.B. Creemers, UMC Utrecht

Prof. dr. R. Lories UZ Leuven (Belgium)

Prof. dr. N. J. J. Verdonschot University of Twnete

Dr. J. Hendriks CellCoTec, B.V.

Prof. dr. L.W.M.M. Terstappen University of Twente

Mesenchymal stem cells as trophic mediators in cartilage regeneration

Ling Wu

Ph.D. Thesis, with references; with summary in English and in Dutch

University of Twente, Enschede, the Netherlands, September 2012.

Copyright (C) 2012 by L. Wu. All rights reserved.

ISNB: 978-90-365-3397-3

The art cover was inspired by traditional Chinese ink painting of orchids and bamboos. One thing in common about art and science is the simplicity. Scientific research is sophisticated; however, scientists have the responsibility to show it in a simple way.

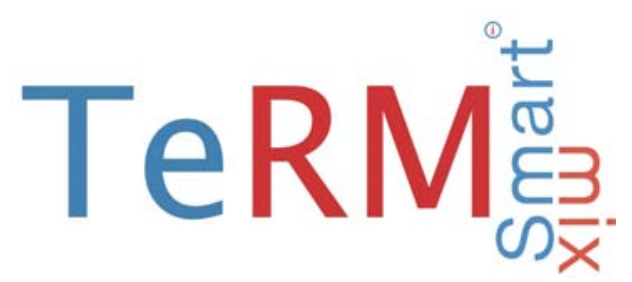

This research is financially supported by the TeRM Smart Mix Program of the Netherlands Ministry of Economic Affairs and the Netherlands Ministry of Education, Culture and Science. 


\title{
MESENCHYMAL STEM CELLS AS \\ TROPHIC MEDIATORS IN CARTILAGE \\ REGENERATION
}

\author{
DISSERTATION \\ to obtain \\ the degree of doctor at the University of Twente, \\ on the authority of the rector magnificus, \\ Prof. Dr. H. Brinksma, \\ on account of the decision of the graduation committee, \\ to be publicly defended \\ on Wednesday, 12th September 2012 at 14:45 \\ by
}

Ling Wu

born on the 16th April 1982

in Sichuan, China 
This dissertation has been approved by:

Promotors: Prof. dr. M. Karperien and Prof. dr. C. A. van Blitterswijk 
To Xiaoqiong and Yuxin 以此论文献给我的家人 

Chapter 1 General introduction and aims $\ldots \ldots \ldots \ldots \ldots \ldots$

Chapter 2 Regeneration of articular cartilage by adipose tissue derived mesenchymal stem cells: perspectives from stem cell biology and molecular medicine $\ldots \ldots \ldots \ldots \ldots \ldots \ldots \ldots \ldots \ldots \ldots \ldots \ldots$

Chapter 3 Trophic effects of mesenchymal stem cells increase chondrocyte proliferation and matrix formation $\ldots \ldots \ldots \ldots \ldots \ldots \ldots$

Chapter 4 Trophic effects of mesenchymal stem cells in chondrocyte cocultures are independent of culture conditions and cell sources ...........................

Chapter 5 Fibroblast Growth Factor -1 is a mesenchymal stem cell secreted factor stimulating chondrocyte proliferation in coculture

Chapter 6 Better cartilage formation in chondrocyte co-cultures with the stromal vascular fraction of adipose tissue than with adipose stem

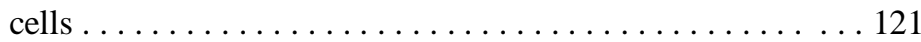

Chapter $7 \quad$ General Discussion .................... 155

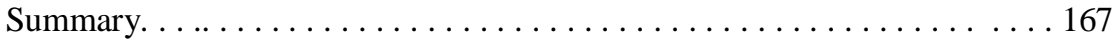

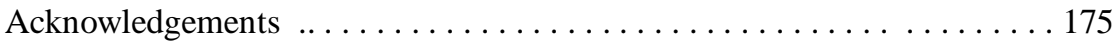

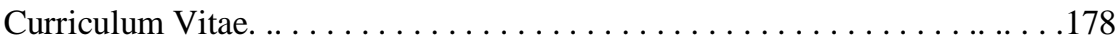

Publication list. ........................... 179 



\section{Chapter 1}

\section{General introduction and aims}

This chapter is an overall introduction to the entire thesis, with aims and outlines for each chapter. 


\section{General introduction and aims}

Autologous Chondrocyte Implantation (ACI) is currently considered as the golden standard for treatment of large-size cartilage defects. ACI requires, however, at least two operative procedures which are separated by several weeks due to the obligatory cell expansion to obtain sufficient cells for implantation. Replacement of chondrocytes by alternative cell sources can potentially reduce the two step procedure to a single step procedure by omitting the cell expansion phase. Hendriks et al., cocultured bovine primary chondrocytes with human expanded chondrocytes, human dermal fibroblasts, mouse embryonic stem cells, mouse-3T3 feeder cells, or human mesenchymal stem cells in cell pellets [1]. Their data indicated that cartilage matrix deposition could be supported by co-culturing chondrocytes with a variety of cell types. In their experimental setup, the co-culture pellets contained approximately $20 \%$ of chondrocytes, but the amount of GAG in co-culture pellets was similar to pure chondrocytes pellets. This synergistic effect of cartilage formation in co-cultures of chondrocytes with other cell types was defined as chondro-induction [2]. The finding of chondro-induction potentially leads to the development of a new cell-based therapy for cartilage regeneration: one step surgery of ACI, in which the necessity for in vitro chondrocyte expansion in laboratory is circumvented.

In this single step procedure, it was proposed that chondrocytes can be isolated, mixed with mesenchymal stem cells, which are also isolated during the same surgical procedure. This mixture of chondrocytes and MSCs is then loaded on a porous scaffold with mechanical properties matching with native cartilage tissue. The procedure is finalized by implantation of the construct into the cartilage defect. Preliminary data shows that the amount of cartilage produced in this procedure equals or is even higher than that formed by a pure chondrocyte population or a pure mesenchymal stem cell population [3-6]. However, developing this surgical procedure from laboratory to clinic needs to meet a few requirements. First of all, fast and efficient isolation of primary chondrocytes and MSCs is essential for performing the surgery in one procedure. Secondly, animal studies are needed to address the safety issues upon scaffolds and cells, as well as efficacy of the single step surgery. In addition, it is as yet unclear what the best source of clinically accessible mesenchymal stem cells is. Upon these questions, 
the mechanism of cross-talk between the chondrocytes and MSCs in co-cultures or coimplantation requires more laboratory studies from the perspective of fundamental science. Elucidating this mechanism helps the application of one step surgery in clinics.

\section{Aims and Outlines of this Thesis}

The aim of this thesis is to uncover the mechanism of cellular interactions between MSCs and chondrocytes in co-culture pellets. There are several questions which are addressed in this thesis: 1) why is extracelluar matrix deposition increased in co-culture pellets of primary chondrocytes and human Mesenchymal Stem Cells; 2) which factors are involved in these interactions; and 3) how can we utilize our knowledge about these interactions to improve clinical practice.

Chapter 2 reviews most up-to-date studies applying adipose derived stem cells (ASCs) in cartilage regeneration research.

Chapter 3 describes the finding of trophic effects in co-culture pellets of MSCs and chondrocytes. We show that increased cartilage formation in pellet cocultures is mainly due to a trophic role of the MSCs in stimulating chondrocyte proliferation and matrix deposition by chondrocytes rather than MSCs actively undergoing chondrogenic differentiation. We provide evidence that this trophic effect is mainly caused by MSC secreted factors.

Chapter 4 expands the findings in chapter 3 to multiple sources of MSCs. Our results demonstrate that trophic effects of MSCs could be a general mechanism by which MSCs from different origins orchestrate tissue function repair.

Chapter 5 focuses on the molecular mechanism of MSCs' trophic effects aiming at the identification of secreted factors that are responsible for the stimulation of chondrocyte proliferation. Our data indicate that MSCs stimulate chondrocyte proliferation in co-culture pellets by secretion of FGF-1 which is strongly increased in co-cultures.

Chapter 6 shows the influence of in vitro expansion on the trophic effects of adipose stem cells (ASCs). Our data indicate that the unexpanded stromal vascular fraction (SVF) of adipose tissue is a better cell source for cartilage regeneration that cultured ASCs. 
In chapter 7, the general discussion, the main conclusions of this thesis are discussed and placed in a broader perspective. In addition, implications for improvement of current cell based cartilage repair technology are discussed.

\section{References}

1. Hendriks JAA, Miclea RL, Schotel R, et al. Primary chondrocytes enhance cartilage tissue formation upon co-culture with a range of cell types. Soft Matter 2010;6:5080-5088.

2. Acharya C, Adesida A, Zajac P, et al. Enhanced chondrocyte proliferation and mesenchymal stromal cells chondrogenesis in coculture pellets mediate improved cartilage formation. J Cell Physiol 2012;227:88-97.

3. Mo XT, Guo SC, Xie HQ, et al. Variations in the ratios of co-cultured mesenchymal stem cells and chondrocytes regulate the expression of cartilaginous and osseous phenotype in alginate constructs. Bone 2009; 45:42-51.

4. Tsuchiya K, Chen G, Ushida T, et al. The effect of coculture of chondrocytes with mesenchymal stem cells on their cartilaginous phenotype in vitro. Materials Science \& Engineering CBiomimetic and Supramolecular Systems 2004;24:6.

5. Hildner F, Concaro S, Peterbauer A, et al. Human adipose-derived stem cells contribute to chondrogenesis in coculture with human articular chondrocytes. Tissue Eng Part A 2009; 15:3961-3969.

6. Vadala G, Studer RK, Sowa G, et al. Coculture of bone marrow mesenchymal stem cells and nucleus pulposus cells modulate gene expression profile without cell fusion. Spine (Phila Pa 1976) 2008;33:870-876. 


\section{Chapter 2}

\section{Regeneration of articular cartilage by adipose tissue derived mesenchymal stem cells: perspectives from stem cell biology and molecular medicine*}

Ling $\mathrm{Wu}^{1}$, Xiaoxiao $\mathrm{Cai}^{2}$, Yunfeng Lin $^{2}$, Marcel Karperien ${ }^{1}$

1. Department of Developmental BioEngineering, MIRA-Institute for Biomedical Technology and Technical Medicine, University of Twente, Enschede, $7522 \mathrm{NB}$, the Netherlands.

2. State Key Laboratory of Oral Diseases, West China College of Stomatology, Sichuan University, Chengdu 610041, P. R. China;

* Manuscript in preparation. 


\begin{abstract}
Adipose-derived stem cells (ASCs) have been discovered for more than a decade. Due to the large numbers of cells that can be harvested with relatively little donor morbidity, they are considered to be an attractive alternative to bone marrow derived mesenchymal stem cells. Consequently, isolation and differentiation of ASCs draw great attention in the research of tissue engineering and regenerative medicine. Cartilage defects cause big therapeutic problems because of their low self-repair capacity. Application of ASCs in cartilage regeneration gives hope to treat cartilage defects with autologous stem cells. In recent years, a lot of studies have been performed to test the possibility of using ASCs to re-construct damaged cartilage tissue. In this paper, we have reviewed the most up-to-date articles utilizing ASCs for cartilage regeneration in basic and translational research. Our topic covers differentiation of adipose tissue derived mesenchymal stem cells into chondrocytes, increased cartilage formation by co-culture of ASCs with chondrocytes and enhancing chondrogenic differentiation of ASCs by gene manipulation.
\end{abstract}




\section{Introduction}

Cartilage defects due to trauma, tumor ablation or age-related abrasion, lead to constant pain and functional limitations of joints and cause serious medical and social problems. It is believed that even small lesions can severely affect the structure and function of articular cartilage and may predispose to the development of osteoarthritis [1]. The reason for this is quite obvious: no vascularization is present in articular cartilage tissues. Therefore, normal events in tissue repair like inflammation and fibrin clot formation do not happen in cartilage defects. Only chondrocyte and synoviocytes which reside in the local environment can fill up the defects by slow proliferation and matrix deposition [2-3]. In cartilage defects deep into the subchondral bone, bone marrow cells as well as blood cells can migrate to the articular surface by bleeding to fill the gaps with rapid proliferation and matrix synthesis [4]. However, the newly synthesized matrix is usually fibrous. And fibrous cartilage is inferior to hyaline cartilage in mechanical properties [5]. Troubled by the poor self-regeneration of cartilage tissue, clinicians and basic scientists have been working for years on new techniques to find the perfect treatment for cartilage defects.

The most popular treatments for cartilage defects nowadays, are micro-drilling and autologous chondrocytes implantation (ACI). In the micro-drilling technique also known as microfracturing, tiny fractures are induced into the subchondral bone plate by drilling small holes which allow blood and bone marrow to seep out in the defect. This creates a blood clot with incorporated pluripotent mesenchymal stem cells (MSCs). These MSCs eventually heal the defect with scar tissue consisting of a mixture of fibrous tissue, fibrocartilage and hyaline-like cartilage [6]. Regarding the clinical outcome, improvements in joint function and pain relief have been reported in $75 \%$ of young patients, with even higher success rates in young athletes [7]. However, the quality of the newly formed cartilage is generally out of control, since it may depend on various factors including the gender and age of the patients, the size and location of the defects, the surgical protocols used, and the post-surgery rehabilitation [8]. In addition, the mechanical properties of scar tissue are inferior compared to native cartilage which may predispose the defected joint to early onset osteoarthritis in the medium to long run. Another treatment called ACI was first introduced by Brittberg et al. in 1994 [9]. The 
rational behind ACI is to fill the cartilage defects with autologous chondrocytes which are expanded in vitro. The classical procedure includes arthroscopic excision of biopsies from low-weight bearing areas of healthy cartilage, isolation and expansion of chondrocytes in the laboratory, and implantation of chondrocyte suspension into the defects which is then covered by a periosteal flap sutured to the surrounding healthy tissues.

Nowadays, new technique called matrix-induced autologous chondrocyte implantiation (MACI) is becoming more popular. Instead of injection into defects as cell suspention, chondrocytes were seeded on a bilayer of porcine-derived type I/type III collagen, after in vitro expansion. The MACI membrane is then secured directly to the defect by fibrin glue without a cover [10]. Clinical studies with a follow-up period of 210 years indicated that $90 \%$ of treated patients developed well-integrated tissue in the defect sites [11]. Despite the success of ACI in clinical practice, there are some drawbacks of this therapeutic method that limit its broader application. One major issue is that the success rate of the procedure severely drops with age limiting the application of ACI to patients under the age of 50 years. Other drawbacks include expensive surgical procedures, donor site morbidity, and dedifferentiation of chondrocytes during in vitro expansion. In vitro expansion is required since relatively large quantities of healthy chondrocytes from the patient are required to fill up the defect site. Replacement of chondrocytes with other cell sources like stem cells gives hope to tackle this problem.

\section{Differentiation of adipose tissue derived mesenchymal stem cells into chondrocytes}

Adipose tissue, like bone marrow, is derived from the embryonic mesenchyme and contains a stroma that can be easily isolated. It was first reported in 2001 , that a group of multipotent cells can be isolated from the stromal vascular fraction (SVF) of collagenase digested human adipose tissue [12]. These cells called adipose tissuederived stromal cells or adipose stem cells (ASCs) can differentiate into adipocytes, osteoblasts, chondrocytes and myocytes under specific culture conditions in vitro [13]. From that point on, many documents have emerged to describe the chondrogenic potential of ASCs isolated from diverse animal models including mouse [14], rat [15], rabbit[16], dog [17] and pig[18]. 


\section{Chondrogenic potential of ASCs}

When cultured in medium containing proper growth factors (TGF $\beta-1$, TGF $\beta-2$, TGF $\beta-3$, BMP-2, BMP-6, or BMP-7), ASCs differentiate into chondrocytes in vitro [14, 19-20]. With a few days pre-conditioning in chondrogenic medium, ASCs could form cartilage tissue in vivo [21]. Unlike bone marrow stromal cells (BMSCs), ASCs can be isolated in large quantities with minimal morbidity and discomfort clinically [22]. In view of these practical advantages, ASCs are an alternative for chondrocytes or BMSCs in cell based cartilage regeneration strategies.

Regarding the application of ASCs in cartilage repair, infra-patellar fat pad (IFP) could be a more attractive clinical source of ASCs. IFP can give rise to cells that fulfill all the criteria of MSCs, including most importantly significant chondrogenic potential [23-25]. It was even reported that ASCs derived from osteoarthritic (OA) IFP showed higher chondrogenic capacity than that of bone marrow MSCs and subcutaneous fat-derived ASCs [26-27]. Moreover, it was reported that chondrogenic potential of IFP derived ASCs was better preserved during in vitro expansion process compared to OA-cartilage derived chondrocytes which rapidly lose their phenotype [28].

\section{Micro-environment needed for cartilage matrix deposition of ASCs}

The differentiation medium required to induce chondrogenic differentiation of ASCs usually contains a cocktail of growth factors. Transforming growth factor- $\beta$ (TGF- $\beta$ ) is considered as the most important component. There are three TGF- $\beta$ isoforms: TGF- $\beta 1,-\beta 2$ and $-\beta 3$. Their distinct roles in embryonic development have been studied intensively in mouse and human [29-31]. However, their differential functions on extracellular matrix (ECM) formation were just discovered recently. Studies showed that TGF- $\beta 3$ and TGF- $\beta 2$ led to significantly higher collagen type II expression and glycosaminoglycans deposition of BMSC than TGF- $\beta 1$ [32]. Cals et al reported that no significant differences in total collagen and glycosaminoglycans (GAGs) formation could be observed among BMSCs cultured in medium containing the three TGF- $\beta$ isoforms respectively [33]. However cells induced by TGF- $\beta 3$ had significantly higher mineralization level than cells cultured in TGF- $\beta 1$ containing medium. Although we did not find any study in which the differences of TGF- $\beta$ isoforms on chondrogenic 


\section{Chapter 2}

differentiation of ASCs were tested, these data suggest that differences between isoforms of TGF- $\beta$ may affect ASCs differentiation and ECM deposition as well.

BMP-6 is another important growth factor commonly used in the differentiation medium. It was reported that BMP-6 when combined with TGF- $\beta$ significantly increased chondrogenesis of ASCs by up-regulating the expression of aggrecan and collagen II with minimal side-effects such as increased collagen type $X$ expression or other characteristics of a hypertrophic phenotype [34]. The mechanism of the synergistic effects of BMP-6 and TGF- $\beta$ is that BMP- 6 could induce the expression of TGF- $\beta$ receptor 1 which is usually not expressed by ASCs [35].

BMP-2 was used as a stimulator for osteogenic differentiation of ASCs [36]. However, BMP-2 was also applied to promote the chondrogenic differentiation of MSCs [37-38]. The cross talk between TGF and BMP signaling suggests an important role of BMP-2 in cartilage matrix deposition [39-40]. Notably, BMP-2 induced chondrogenic differentiation of MSCs would eventually lead to hypertrophy and endochondral-ossification [41-42].

BMP-4 is traditionally considered as a trigger of adipogenic differentiation of embryonic stem cells [43]. A recent article presented BMP-4 as a promising growth factor for ASCs' in vitro expansion since a low dose of BMP-4 increased their viability and maintained their multipotency [44]. Addition of BMP-4 in the differentiation medium significantly enhanced the chondrogenic phenotype of ASCs compared to TGF$\beta 1$ alone [45].

The role of BMP-7 in ASCs differentiation is not as clearly defined as other BMPs. On one hand, BMP-7 has been shown to be an important regulator of brown fat adipogenesis and energy expenditure [46]; on the other hand, it is also commonly used in bone tissue engineering to promote healing of critical size bone defects [47-49]. To make it even more complex, there are reports claiming that BMP-7 could initiate a more chondrogenic phenotype in ASCs than BMP-2 [19]. It looks like BMP-7 is involved in all the three mesenchymal lineages and might play multiple roles in the differentiation of ASCs.

In many studies, serum free medium was used for chondrogenic differentiation. It was reported that serum free medium maintained the expression of Sox 9 in 
chondrocytes during in vitro expansion and sustained their phenotype, while serum caused the de-differentiation of chondrocytes [50]. Another report claimed that fetal bovine serum (FBS) in the differentiation medium inhibited the production of glycosaminoglycans (GAGs) and type II collagens in synovial cells [51]. However, the negative effects of serum on chondrogenic differentiation of ASCs appears to be weak, since differentiation of ASCs towards chondrocytes was observe with the presence of serum $[14,52]$.

Conventionally, chondrocytes or MSCs must be placed in a three dimensional culture environment such as a micro-mass or a pellet culture before they start depositing cartilage matrix [53]. One misconception is that 3D (3 dimensional) culture is required for chondrogenic differentiation of ASCs. Actually, chondrogenic differentiation of ASCs involves two biological events: commitment into chondrogenic lineage and deposition of cartilage matrix. There is ample evidence showing that 3D culture environment is not essential for chondrogenic commitment of ASCs. In vitro induction of ASCs in 2D (2 dimensional) was sufficient to make these cells express chondrogenic genes and form cartilage tissue in nude mice [21,54].

\section{Molecular cascades in ASCs during chondrogenic differentiation}

We previously identified a group of Osteo-adipo progenitors (OAPs) in stromal vascular fraction (SVF) from adipose tissue [55]. This group of cells possess bidirectional differentiation potential which are derived from the Scal-1 negative cell population. They simultaneously express adipogenic and osteogenic genes (RUNX2 and PPAR- $\gamma$ ). Interestingly, PPAR- $\gamma$ moved from cytoplasm to the nucleus when OAPs differentiated into adipocytes, while RUNX2 stayed in the cytoplasm. In contrast, RUNX2 moved from cytoplasm to the nucleus when OAPs differentiated into osteoblast, while PPAR- $\gamma$ remained in the cytoplasm [55]. This paper together with other studies [56-58] demonstrated an interesting reciprocal relationship between osteogenesis and adipogenesis: osteogenic induction enhanced expression of osteogenic genes and inhibited expression of adipogenic genes, while adipogenic induction enhanced expression of adipogenic genes and inhibited expression of osteogenic genes.

When ASCs lost their potential to adipogenic lineage, they seem to be able to differentiate into both chondrocytes and osteoblasts. From a developmental point of 


\section{Chapter 2}

view, osteoblasts and chondrocytes share the same progenitor [59]. During endochondral ossification, mesenchymal progenitors first differentiate into an intermediate bipotential progenitor cell that can give rise to both the chondrocytes which give rise to primary growth plate and the osteoblasts in the bone collar. After a period of proliferation, growth plate chondrocytes become hypertrophic, die and are replaced by osteoblasts depositing bone on the cartilaginous matrix [60]. Osteochondral progenitors are not only observed during development, but are also found in vitro. A number of bipotential cell lines have been described to differentiate into both the osteogenic and chondrogenic lineages simultaneously [61-62]. Reciprocal relationship between osteogenesis and chondrogenesis was also found in osteochondral progenitors. Hypertrophic differentiation of chondrocytes is tightly controlled by the balance of Sox9 and Runx2: Sox9 preserves the chondrogenic phenotype, while Runx2 accelerates hypertrophic differentiation. RunX2 also acts as the master transcription regulator of osteoblastic differentiation [63-64].

Once ASCs are committed to the chondrogenic lineage, molecular events become clear and simple. Cells stably express Sox9, and then Sox9 triggers the expressions of cartilage matrix proteins, including collagen type II (COL II), collagen type IX (COLIX), aggrecan (ACAN), and cartilage oligomeric matrix protein (COMP) [21]. Then a group of cytokines is secreted by mature chondroyctes to maintain the expression of Sox 9 and other chondrogenic marker genes such as COL II and ACAN [65]. The molecular events regulating the step-wise differentiation from tri-potential ASCs into bi-potential osteochondral progenitors and then into committed chondrocytes are summarized in figure 1.

\section{Increased cartilage formation by Co-culture of ASCs with chondrocytes}

Cartilage is a unique tissue in which only one cell population resides. Cellular interactions between chondrocytes and other cell types are rare occasions that can only occur at the superficial zone of cartilage and at the interphase between cartilage and the subchondral bone. When co-culture was first introduced into the cartilage field as a research tool [66], it was mainly used to study the pathophysiology of rheumatoidarthritis and osteoarthritis by investigating the cross-talk between chondrocytes on one hand and synoviocytes on the other [67], or between chondrocytes and osteoblasts [68]. 
Only recently, it has become clear that co-culture has great potential in cartilage regeneration [69].

\section{Synergistic effects in co-culture of ASCs and chondrocytes}

To reduce the cell number need for ACI, chondrocytes may be partially replaced by other more easily obtained cell types. Tsuchiya et. al, first reported that coculture of BMSCs and articular chondrocytes enhanced matrix production [70]. The synergistic effects of co-culture were confirmed by other researchers in similar coculture models [71-72]. Meanwhile, increased cartilage matrix formation was also reported in co-culture of chondrocytes with ASCs [73].

To explain the mechanism of increased cartilage formation in co-cultures, two hypotheses have been proposed: 1) increased cartilage formation is due to chondrogenic differentiation of MSCs triggered by signals from chondrocytes; 2) increased cartilage matrix is a result of enhanced activity of chondrocytes stimulated by MSCs. Two hypotheses are illustrated in figure 2.

\section{Chondrocytes promote differentiation of ASCs}

It was suggested that beneficial effects of co-culturing chondrocytes with MSCs are largely due to the differentiation of MSCs into chondrocytes. Soluble factors released from chondrocytes have been shown to support chondrogenesis in an indirect co-culture model of human embryonic stem cells (hESCs) and primary chondrocytes by significantly enhancing the expression of proteoglycans, collagen I and II [74]. Conditioned medium of chondrocytes could induce osteo-chondrogenic differentiation of BMSCs [75]. It was also reported that co-culture of BMSCs and chondrocytes in a 3D environment induced chondrogenic gene expression in BMSCs [76]. In a trans-well co-culture system, chondrogenic differentiation of BMSCs is increased by chondrocytes [77]. More specifically, several studies revealed that ASC could respond to soluble factors released by nuclear pulposus cells by up-regulating cartilage-specific gene expression such as of COL II and aggrecan [78-80]. A conflicting study reported that direct cell-cell contact was required for the differentiation of BMScs when co-cultured with nucleus pulposus cells [81-82]. Nevertheless, many studies so far indicate secreted soluble factors may be responsible for the differentiation of BMSCs in co-culture with MSCs. 


\section{Chapter 2}

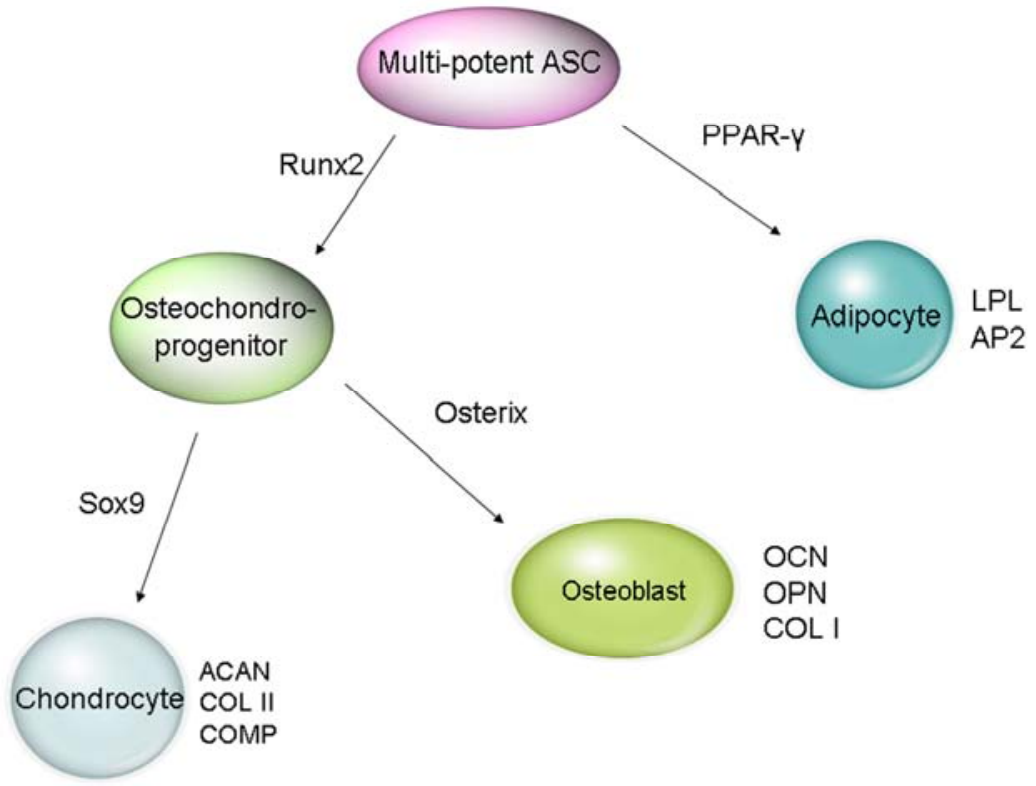

Figure 1; Schematic representation of molecular events during chondrogenic differentiation of ASCs. LPL, lipoprotein lipase; AP2, adipocyte fatty acid-binding protein 2; OCN, osteocalcin; OPN, osteopotin; COL1, collagen I, ACAN, aggrecan; COL2, collagen II, GAG, glycosaminoglycans.

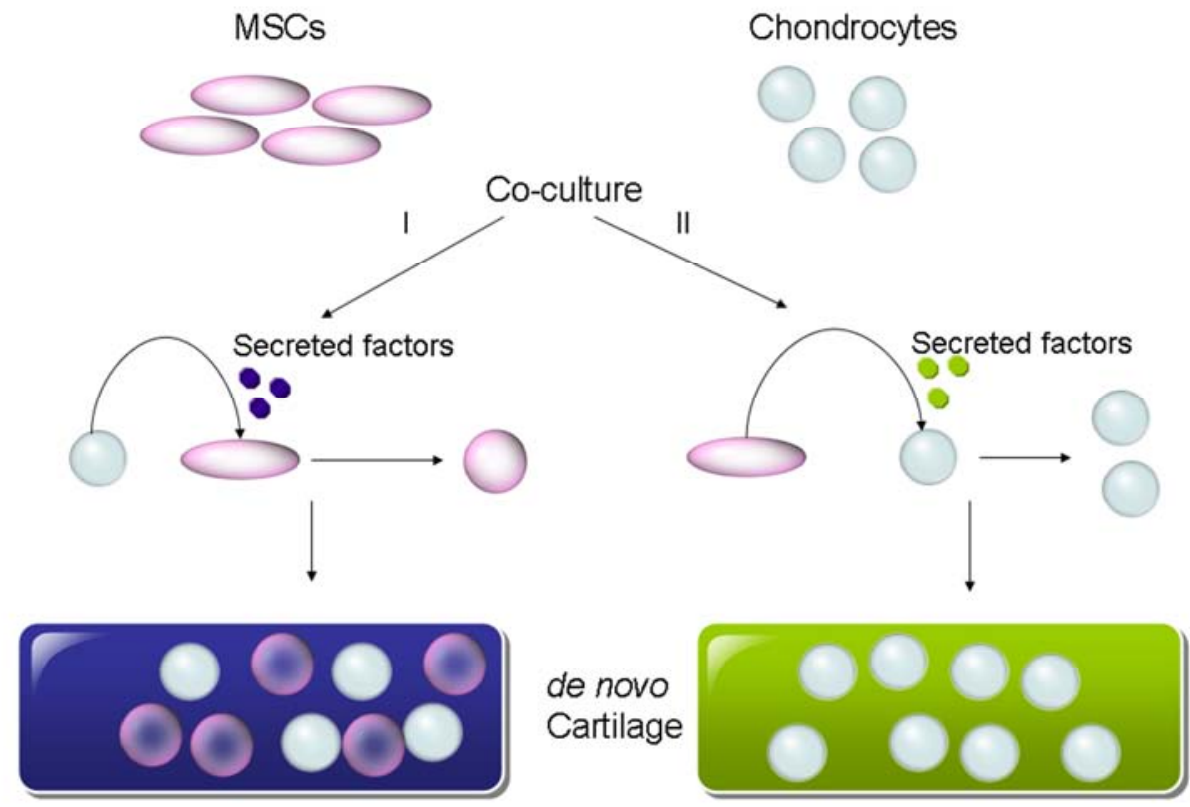

Figure 2: Two hypotheses have been proposed to explain the mechanism of increased cartilage formation in co-cultures of MSCs and chondrocytes 


\section{Trophic effects of MSCs}

In a recently published work, we tracked the two cell populations by using a xenogenic co-culture model of human MSCs and bovine chondrocytes [83]. Their contributions to cartilage matrix formation were therefore separately studied. Our data showed a significant decrease of MSCs in co-culture pellets, resulting in an almost homogeneous cartilage tissue. Thus the beneficial effect of co-culture is largely due to increased chondrocyte proliferation and matrix formation. Chondrogenic differentiation MSCs was shown to be a minor contribution to cartilage formation. Furthermore, these observations are not specific to certain species (combination) or donors. It's the first time a trophic role of MSCs has been demonstrated in stimulating chondrocyte proliferation and matrix production.

Arnold Caplan first proposed MSCs as a trophic mediator for tissue repair [84]. Term TROPHIC traditionally refers to the non-neurotransmitters bioactive molecules produced by nerve terminals in neurology [85]. When first being introduced, trophic effect referred to the effects that MSCs secrete factors that stimulate releasing of functional bioactive factors from surrounding cells [84]. Its definition then expanded to the MSC produced factors that promote cell viability, proliferation, and matrix production in the surrounding environment. The picture has been changed about the roles MSCs played in tissue repair since the introduction of trophic effects into MSCs research. Based on the first pioneer studies, people tend to believe that MSCs repair damaged tissues by differentiating into specific cell types and replacing lost cells [86]. But now, more and more researchers considered the trophic roles of the MSC as more important feature of MSCs in tissue repair [87]. Examples include MSCs improved gain of coordinated functions into brain stroked rats without differentiating into any neuronal related cell type [88] and MSCs stimulated cardiomyocyte proliferation [89] and vascular regeneration [90].

As illustrated by recent co-culture studies [83, 91], the trophic effects of MSCs in cartilage regeneration can be dissected into several layers: 1) MSCs promoted extracellular matrix formation of chondrocytes; 2) MSCs increase proliferation of chondrocytes; 3) MSCs died overtime in the co-culture with chondrocytes. Furthermore, our follow-up study demonstrated that the trophic effects MSCs in co-culture pellets 
stimulating cartilage formation are independent of the culture conditions or MSCs origins [92]. Co-culture pellets grow in medium stimulating chondrogenic differentiation gave similar results as pellets cultured in proliferation medium. The origins of the MSCs are also proved to be unimportant for their trophic effects since coculturing chondrocytes with MSCs isolated from bone marrow, adipose tissue and synovial membrane all showed similar results. This implies that it's a very general observation that the MSCs play as trophic mediators in co-cultures with chondrocytes.

\section{Enhanced chondrogenic differentiation of ASCs by gene manipulation}

Besides co-culture ASCs with chondrocytes, over-expression of regulatory genes in ASCs is another strategy to enhance chondrogenic differentiation [93]. Our previous studies have shown that ASCs are good cell source for genetic modification $[36,94-95]$. Genes related to muscle-skeleton development have been introduced into ASCs to improve the differentiation of ASCs [96-97]. On the list of genes involved in cartilage development, there are generally two groups of genes which are potentially useful for genetic manipulation to boostcartilage regeneration [98]. These are genes encoding anabolic growth factors, such as TGF- $\beta$, BMPs and Insulin-like Growth Factor (IGF), and transcription factors like Sox-5, -6 and -9 that control chondrogenesis.

\section{Growth factors: TGF- $\beta$}

TGF- $\beta 1$ has been regarded as the most powerful chondrogenic growth factor, which induces significant chondrogenic phenotype of ASCs both in vitro and in vivo [14, 21]. Guo T et al., reported that a plasmid DNA encoding TGF- $\beta 1$ could be entrapped into a chitosan-gelatin based biomaterial to enhance extracellular matrix deposition of chondrocytes which were incorporated in the same materials [99]. In a similar study, Guo X et el., used a sightly different stratigy in which plasmid TGF- $\beta 1$ was tranfected into BMSCs, then transfected cells were applied to repair full-thickness articular cartilage defects in a rabbit model [100]. There are no reports on expressing TGF- $\beta 1$ or TGF- $\beta 3$ in ASCs. In contrast, TGF- $\beta 2$ transduced ASCs have been used. In these studies PLGA/alginate compound materials have been used to potentiate the differentiation of the genetically manipulated ASCs [101-102]. It's also been demonstrated that TGF- $\beta 2$ transfected ASCs could repair articular cartilage defects in 
rabbits [103].

\section{Growth factors: BMPs and others}

Exogenic expression of BMPs in ASCs normally leads to osteogenic differentiation. For example, BMP-2 transfected ASCs developed an osteoblastic phenotype and after loading in an alginate gel were used to repair critical size cranial defects in rat models [36]. BMP-7 was also transduced into ASCs to promote bone formation both in vitro and in vivo [104]. However, there are some BMPs found to induce cartilage matrix formation when over-expressed in pluoripotent stem cells or dedifferentiated chondrocytes. These BMPs might be useful to boost cartilage formation when overexpressed in ASCs. Kuroda et al., reported that BMP-4 transduced muscle derived stem cells (MDSCs) acquired chondrocyte-like characteristics in vitro and formed better cartilage in knee repair models in rats [105]. The repairing results could even be better if BMP-4 was co-tranduced with sFit-1 [106]. Lin et al., demonstrated that BMP-4 could induce re-differentiation of chondrocytes which lost their typical phenotype [107]. The only BMP that has been ectopically expressed in ASCs is BMP-6, due to the special effects of BMP-6 that induces the expression of TGF- $\beta$ receptor 1 on ASCs [35]. Diekman et al., reported a model of alginate beads to culture ASCs transfected with a pcDNA3-BMP-6 construct and confirmed the induction of chondrogenic differentiation of ASCs [108].

Other growth factors that were considered for over-expression in ASCs for cartilage tissue engineering purposes are IFG-1, fibroblast growth factors (FGF) and epidermal growth factors (EGF). Results from a previous study suggest that dynamic compression combined with IGF-1 over-expression could benefit cartilage tissue formation of ASCs seeded in chitosan/gelatin scaffolds [109]. Although FGF and EGF are believed to benefit the proliferation of ASCs while keeping their chondrogenic potential [110-111], no transgenic studies have ever been conducted in ASCs with these two groups of factors so far.

\section{Transcription factors: Sox 9 and its family members}

Sox 9 is considered as the "master regulator" of chondrogenic differentiation [112], since it directly controls the synthesis of collagen type II and other ECM matrix in cartilage tissue [113-114]. A few researchers used adenovirus to deliver exogenic Sox 


\section{Chapter 2}

9 gene in chondrocytes and disc cells to increase the deposition of cartilage specific ECM [115]. With respect to tissue engineering, Sox 9 was over-expressed in BMSCs by adenoviral transduction [116-117]. Infected BMSCs express higher level of Collagen II than cells without transduction. Recently researchers started expressing exogenous Sox 9 in ASCs in an attempt to boost cartilage matrix formation. Yang et al. infected ASCs with a retrovirus expressing Sox 9 [118]. In this study, they found that collagen II and proteoglycan production was increased in Sox 9 engineered ASCs. Furthermore, coculture of Sox-9 transduced ASCs and nuclear pulposus cells in alginate beads resulted in an increase of collagen II and GAGs production. A new trend in these studies is to cotransfect ASCs with SOX Trio (Sox 5, 6 and 9 genes), since Sox 5 and 6 are believed to cooperate with Sox 9 in cartilage development [119-120]. Studies showed that transfection of SOX Trio initiated the differentiation of ASCs into chondrocyte-like cells both in vitro and in vivo [121]. It was even reported that SOX Trio retroviraltransduced ASCs seeded in fibrin gel promoted the healing of osteochondral defects and prevented the progression of experimental osteoarthritis in a rat model [122]. Besides plasmid transfection and viral transduction, the delivery method could also be seeding ASCs on PLGA hydrogel incorporated with the pcDNA vector expressing SOX Trio. This method has been successfully used to treat osteochondral defects on the patellar groove of a rabbit model [123].

\section{Conclusion}

Many efforts have been made to improve cartilage regeneration during the last few decades. Advances have been achieved to efficiently differentiate ASCs into chondrocyte-like cells. These findings can be potentially translated into stem cell-based therapies for treating large size cartilage defects. Achievements in this field have shown a wide range of prospects and promise to support cartilage regeneration in the future. 


\section{References}

1. Alford JW, Cole BJ. Cartilage restoration, part 1: basic science, historical perspective, patient evaluation, and treatment options. Am J Sports Med 2005;33:295-306.

2. Vincent T, Hermansson M, Bolton M, et al. Basic FGF mediates an immediate response of articular cartilage to mechanical injury. Proc Natl Acad Sci U S A 2002;99:8259-8264.

3. Mankin HJ. The response of articular cartilage to mechanical injury. J Bone Joint Surg Am 1982;64:460-466.

4. Furukawa T, Eyre DR, Koide S, et al. Biochemical studies on repair cartilage resurfacing experimental defects in the rabbit knee. J Bone Joint Surg Am 1980;62:79-89.

5. Nehrer S, Spector M, Minas T. Histologic analysis of tissue after failed cartilage repair procedures. Clin Orthop Relat Res 1999:149-162.

6. Gilbert JE. Current treatment options for the restoration of articular cartilage. Am J Knee Surg 1998;11:42-46.

7. Sledge SL. Microfracture techniques in the treatment of osteochondral injuries. Clin Sports Med 2001;20:365-377.

8. Alford JW, Cole BJ. Cartilage restoration, part 2: techniques, outcomes, and future directions. Am J Sports Med 2005;33:443-460.

9. Brittberg M, Lindahl A, Nilsson A, et al. Treatment of deep cartilage defects in the knee with autologous chondrocyte transplantation. N Engl J Med 1994;331:889-895.

10. Bartlett W, Skinner JA, Gooding CR, et al. Autologous chondrocyte implantation versus matrixinduced autologous chondrocyte implantation for osteochondral defects of the knee: a prospective, randomised study. J Bone Joint Surg Br 2005;87:640-645.

11. Peterson L, Minas T, Brittberg M, et al. Treatment of osteochondritis dissecans of the knee with autologous chondrocyte transplantation: results at two to ten years. J Bone Joint Surg Am 2003;85-A Suppl 2:17-24.

12. Zuk PA, Zhu M, Mizuno H, et al. Multilineage cells from human adipose tissue: implications for cell-based therapies. Tissue Eng 2001;7:211-228.

13. Zuk PA, Zhu M, Ashjian P, et al. Human adipose tissue is a source of multipotent stem cells. Mol Biol Cell 2002;13:4279-4295.

14. Lin Y, Tian W, Chen X, et al. Expression of exogenous or endogenous green fluorescent protein in adipose tissue-derived stromal cells during chondrogenic differentiation. Mol Cell Biochem 2005;277:181-190.

15. Lopez MJ, Spencer ND. In vitro adult rat adipose tissue-derived stromal cell isolation and differentiation. Methods Mol Biol 2011;702:37-46.

16. Han Y, Wei Y, Wang S, et al. Enhanced chondrogenesis of adipose-derived stem cells by the controlled release of transforming growth factor-beta1 from hybrid microspheres. Gerontology 2009;55:592-599.

17. Reich CM, Raabe O, Wenisch S, et al. Isolation, culture and chondrogenic differentiation of canine adipose tissue- and bone marrow-derived mesenchymal stem cells-a comparative study. Vet Res Commun 2012.

18. Wang KH, Kao AP, Wangchen $\mathrm{H}$, et al. Optimizing proliferation and characterization of multipotent stem cells from porcine adipose tissue. Biotechnol Appl Biochem 2008;51:159-166.

19. Knippenberg M, Helder MN, Zandieh Doulabi B, et al. Osteogenesis versus chondrogenesis by BMP-2 and BMP-7 in adipose stem cells. Biochem Biophys Res Commun 2006;342:902-908.

20. Mehlhorn AT, Niemeyer P, Kaschte K, et al. Differential effects of BMP-2 and TGF-beta1 on chondrogenic differentiation of adipose derived stem cells. Cell Prolif 2007;40:809-823.

21. Lin Y, Luo E, Chen X, et al. Molecular and cellular characterization during chondrogenic differentiation of adipose tissue-derived stromal cells in vitro and cartilage formation in vivo. $\mathbf{J}$ Cell Mol Med 2005;9:929-939.

22. Parker AM, Katz AJ. Adipose-derived stem cells for the regeneration of damaged tissues. Expert Opin Biol Ther 2006;6:567-578.

23. Dragoo JL, Samimi B, Zhu M, et al. Tissue-engineered cartilage and bone using stem cells from human infrapatellar fat pads. J Bone Joint Surg Br 2003;85:740-747.

24. Khan WS, Tew SR, Adesida AB, et al. Human infrapatellar fat pad-derived stem cells express the pericyte marker 3G5 and show enhanced chondrogenesis after expansion in fibroblast growth factor-2. Arthritis Res Ther 2008;10:R74. 


\section{Chapter 2}

25. Buckley CT, Vinardell T, Thorpe SD, et al. Functional properties of cartilaginous tissues engineered from infrapatellar fat pad-derived mesenchymal stem cells. J Biomech 2010;43:920926.

26. Mochizuki T, Muneta T, Sakaguchi Y, et al. Higher chondrogenic potential of fibrous synoviumand adipose synovium-derived cells compared with subcutaneous fat-derived cells: distinguishing properties of mesenchymal stem cells in humans. Arthritis Rheum 2006;54:843-853.

27. Sakaguchi Y, Sekiya I, Yagishita K, et al. Comparison of human stem cells derived from various mesenchymal tissues: superiority of synovium as a cell source. Arthritis Rheum 2005;52:25212529.

28. English A, Jones EA, Corscadden D, et al. A comparative assessment of cartilage and joint fat pad as a potential source of cells for autologous therapy development in knee osteoarthritis. Rheumatology (Oxford) 2007;46:1676-1683.

29. Gatherer D, Ten Dijke P, Baird DT, et al. Expression of TGF-beta isoforms during first trimester human embryogenesis. Development 1990;110:445-460.

30. Millan FA, Denhez F, Kondaiah P, et al. Embryonic gene expression patterns of TGF beta 1, beta 2 and beta 3 suggest different developmental functions in vivo. Development 1991;111:131-143.

31. Schmid P, Cox D, Bilbe G, et al. Differential expression of TGF beta 1, beta 2 and beta 3 genes during mouse embryogenesis. Development 1991;111:117-130.

32. Barry F, Boynton RE, Liu B, et al. Chondrogenic differentiation of mesenchymal stem cells from bone marrow: differentiation-dependent gene expression of matrix components. Exp Cell Res 2001;268:189-200.

33. Cals FL, Hellingman CA, Koevoet W, et al. Effects of transforming growth factor-beta subtypes on in vitro cartilage production and mineralization of human bone marrow stromal-derived mesenchymal stem cells. J Tissue Eng Regen Med 2012;6:68-76.

34. Estes BT, Wu AW, Guilak F. Potent induction of chondrocytic differentiation of human adiposederived adult stem cells by bone morphogenetic protein 6. Arthritis Rheum 2006;54:1222-1232.

35. Hennig T, Lorenz H, Thiel A, et al. Reduced chondrogenic potential of adipose tissue derived stromal cells correlates with an altered TGFbeta receptor and BMP profile and is overcome by BMP-6. J Cell Physiol 2007;211:682-691.

36. Lin Y, Tang W, Wu L, et al. Bone regeneration by BMP-2 enhanced adipose stem cells loading on alginate gel. Histochem Cell Biol 2008;129:203-210.

37. Noth U, Rackwitz L, Heymer A, et al. Chondrogenic differentiation of human mesenchymal stem cells in collagen type I hydrogels. J Biomed Mater Res A 2007;83:626-635.

38. Kurth T, Hedbom E, Shintani N, et al. Chondrogenic potential of human synovial mesenchymal stem cells in alginate. Osteoarthritis Cartilage 2007;15:1178-1189.

39. Keller B, Yang T, Chen Y, et al. Interaction of TGFbeta and BMP signaling pathways during chondrogenesis. PLoS One 2011;6:e16421.

40. Luyten FP, Yu YM, Yanagishita M, et al. Natural bovine osteogenin and recombinant human bone morphogenetic protein-2B are equipotent in the maintenance of proteoglycans in bovine articular cartilage explant cultures. J Biol Chem 1992;267:3691-3695.

41. Carlberg AL, Pucci B, Rallapalli R, et al. Efficient chondrogenic differentiation of mesenchymal cells in micromass culture by retroviral gene transfer of BMP-2. Differentiation 2001;67:128-138.

42. Steinert AF, Proffen B, Kunz M, et al. Hypertrophy is induced during the in vitro chondrogenic differentiation of human mesenchymal stem cells by bone morphogenetic protein-2 and bone morphogenetic protein-4 gene transfer. Arthritis Res Ther 2009;11:R148.

43. Taha MF, Valojerdi MR, Mowla SJ. Effect of bone morphogenetic protein-4 (BMP-4) on adipocyte differentiation from mouse embryonic stem cells. Anat Histol Embryol 2006;35:271-278.

44. Vicente Lopez MA, Vazquez Garcia MN, Entrena A, et al. Low doses of bone morphogenetic protein 4 increase the survival of human adipose-derived stem cells maintaining their stemness and multipotency. Stem Cells Dev 2011;20:1011-1019.

45. Kim BS, Kang KS, Kang SK. Soluble factors from ASCs effectively direct control of chondrogenic fate. Cell Prolif 2010;43:249-261.

46. Tseng YH, Kokkotou E, Schulz TJ, et al. New role of bone morphogenetic protein 7 in brown adipogenesis and energy expenditure. Nature 2008;454:1000-1004.

47. Koh JT, Zhao Z, Wang Z, et al. Combinatorial gene therapy with BMP2/7 enhances cranial bone regeneration. J Dent Res 2008;87:845-849.

48. Yang M, Ma QJ, Dang GT, et al. In vitro and in vivo induction of bone formation based on ex vivo gene therapy using rat adipose-derived adult stem cells expressing BMP-7. Cytotherapy 
2005; 7:273-281.

49. Zhu L, Chuanchang D, Wei L, et al. Enhanced healing of goat femur-defect using BMP7 genemodified BMSCs and load-bearing tissue-engineered bone. J Orthop Res 2010;28:412-418.

50. Malpeli M, Randazzo N, Cancedda R, et al. Serum-free growth medium sustains commitment of human articular chondrocyte through maintenance of Sox9 expression. Tissue Eng 2004;10:145155 .

51. Bilgen B, Orsini E, Aaron RK, et al. FBS suppresses TGF-beta1-induced chondrogenesis in synoviocyte pellet cultures while dexamethasone and dynamic stimuli are beneficial. J Tissue Eng Regen Med 2007;1:436-442.

52. Ogawa R, Mizuno H, Hyakusoku H, et al. Chondrogenic and osteogenic differentiation of adiposederived stem cells isolated from GFP transgenic mice. J Nihon Med Sch 2004;71:240-241.

53. Koch RJ, Gorti GK. Tissue engineering with chondrocytes. Facial Plast Surg 2002;18:59-68.

54. Merceron C, Portron S, Masson M, et al. The effect of two and three dimensional cell culture on the chondrogenic potential of human adipose-derived mesenchymal stem cells after subcutaneous transplantation with an injectable hydrogel. Cell Transplant 2011.

55. Lin YF, Jing W, Wu L, et al. Identification of osteo-adipo progenitor cells in fat tissue. Cell Prolif 2008;41:803-812.

56. Backesjo CM, Li Y, Lindgren U, et al. Activation of Sirt1 decreases adipocyte formation during osteoblast differentiation of mesenchymal stem cells. J Bone Miner Res 2006;21:993-1002.

57. Enomoto H, Furuichi T, Zanma A, et al. Runx2 deficiency in chondrocytes causes adipogenic changes in vitro. J Cell Sci 2004;117:417-425.

58. Heim M, Frank O, Kampmann G, et al. The phytoestrogen genistein enhances osteogenesis and represses adipogenic differentiation of human primary bone marrow stromal cells. Endocrinology 2004; $145: 848-859$.

59. Zou L, Zou X, Li H, et al. Molecular mechanism of osteochondroprogenitor fate determination during bone formation. Adv Exp Med Biol 2006;585:431-441.

60. Mackie EJ, Ahmed YA, Tatarczuch L, et al. Endochondral ossification: how cartilage is converted into bone in the developing skeleton. Int J Biochem Cell Biol 2008;40:46-62.

61. Tominaga H, Maeda S, Miyoshi H, et al. Expression of osterix inhibits bone morphogenetic protein-induced chondrogenic differentiation of mesenchymal progenitor cells. J Bone Miner Metab 2009;27:36-45.

62. Grigoriadis AE, Heersche JN, Aubin JE. Continuously growing bipotential and monopotential myogenic, adipogenic, and chondrogenic subclones isolated from the multipotential RCJ 3.1 clonal cell line. Dev Biol 1990;142:313-318.

63. Dy P, Wang W, Bhattaram P, et al. Sox9 directs hypertrophic maturation and blocks osteoblast differentiation of growth plate chondrocytes. Dev Cell 2012;22:597-609.

64. Ding M, Lu Y, Abbassi S, et al. Targeting Runx2 expression in hypertrophic chondrocytes impairs endochondral ossification during early skeletal development. J Cell Physiol 2011.

65. Polacek M, Bruun JA, Elvenes J, et al. The secretory profiles of cultured human articular chondrocytes and mesenchymal stem cells: implications for autologous cell transplantation strategies. Cell Transplant 2011;20:1381-1393.

66. Goldring SR, Dayer JM, Krane SM. Rheumatoid synovial cell hormone responses modulated by cell-cell interactions. Inflammation 1984;8:107-121.

67. Lubke C, Ringe J, Krenn V, et al. Growth characterization of neo porcine cartilage pellets and their use in an interactive culture model. Osteoarthritis Cartilage 2005;13:478-487.

68. Sanchez C, Deberg MA, Piccardi N, et al. Subchondral bone osteoblasts induce phenotypic changes in human osteoarthritic chondrocytes. Osteoarthritis Cartilage 2005; 13:988-997.

69. Hendriks J, Riesle J, van Blitterswijk CA. Co-culture in cartilage tissue engineering. J Tissue Eng Regen Med 2007;1:170-178.

70. Tsuchiya K, Chen G, Ushida T, et al. The effect of coculture of chondrocytes with mesenchymal stem cells on their cartilaginous phenotype in vitro. Materials Science \& Engineering CBiomimetic and Supramolecular Systems 2004;24:6.

71. Hendriks JAA, Miclea RL, Schotel R, et al. Primary chondrocytes enhance cartilage tissue formation upon co-culture with a range of cell types. Soft Matter 2010;6:5080-5088.

72. Mo XT, Guo SC, Xie HQ, et al. Variations in the ratios of co-cultured mesenchymal stem cells and chondrocytes regulate the expression of cartilaginous and osseous phenotype in alginate constructs. Bone 2009; 45:42-51.

73. Hildner F, Concaro S, Peterbauer A, et al. Human adipose-derived stem cells contribute to 


\section{Chapter 2}

chondrogenesis in coculture with human articular chondrocytes. Tissue Eng Part A 2009;15:39613969.

74. Vats A, Bielby RC, Tolley N, et al. Chondrogenic differentiation of human embryonic stem cells: the effect of the micro-environment. Tissue Eng 2006;12:1687-1697.

75. Hwang NS, Varghese S, Puleo C, et al. Morphogenetic signals from chondrocytes promote chondrogenic and osteogenic differentiation of mesenchymal stem cells. J Cell Physiol 2007;212:281-284.

76. Vadala G, Studer RK, Sowa G, et al. Coculture of bone marrow mesenchymal stem cells and nucleus pulposus cells modulate gene expression profile without cell fusion. Spine (Phila Pa 1976) 2008;33:870-876.

77. Chen WH, Lai MT, Wu AT, et al. In vitro stage-specific chondrogenesis of mesenchymal stem cells committed to chondrocytes. Arthritis Rheum 2009;60:450-459.

78. Lu ZF, Zandieh Doulabi B, Wuisman PI, et al. Differentiation of adipose stem cells by nucleus pulposus cells: configuration effect. Biochem Biophys Res Commun 2007;359:991-996.

79. Li X, Lee JP, Balian G, et al. Modulation of chondrocytic properties of fat-derived mesenchymal cells in co-cultures with nucleus pulposus. Connect Tissue Res 2005;46:75-82.

80. Lu ZF, Doulabi BZ, Wuisman PI, et al. Influence of collagen type II and nucleus pulposus cells on aggregation and differentiation of adipose tissue-derived stem cells. J Cell Mol Med 2008;12:2812-2822.

81. Yamamoto Y, Mochida J, Sakai D, et al. Upregulation of the viability of nucleus pulposus cells by bone marrow-derived stromal cells: significance of direct cell-to-cell contact in coculture system. Spine (Phila Pa 1976) 2004;29:1508-1514.

82. Richardson SM, Walker RV, Parker S, et al. Intervertebral disc cell-mediated mesenchymal stem cell differentiation. Stem Cells 2006;24:707-716.

83. Wu L, Leijten JC, Georgi N, et al. Trophic effects of mesenchymal stem cells increase chondrocyte proliferation and matrix formation. Tissue Eng Part A 2011;17:1425-1436.

84. Caplan AI, Dennis JE. Mesenchymal stem cells as trophic mediators. J Cell Biochem 2006;98:1076-1084.

85. Singer M. Trophic functions of the neuron. VI. Other trophic systems. Neurotrophic control of limb regeneration in the newt. Ann N Y Acad Sci 1974;228:308-322.

86. Bruder SP, Fink DJ, Caplan AI. Mesenchymal stem cells in bone development, bone repair, and skeletal regeneration therapy. J Cell Biochem 1994;56:283-294.

87. Kassis I, Vaknin-Dembinsky A, Karussis D. Bone marrow mesenchymal stem cells: agents of immunomodulation and neuroprotection. Curr Stem Cell Res Ther 2011;6:63-68.

88. Li Y, Chen J, Zhang CL, et al. Gliosis and brain remodeling after treatment of stroke in rats with marrow stromal cells. Glia 2005;49:407-417.

89. Sassoli C, Pini A, Mazzanti B, et al. Mesenchymal stromal cells affect cardiomyocyte growth through juxtacrine Notch-1/Jagged1 signaling and paracrine mechanisms: Clues for cardiac regeneration. J Mol Cell Cardiol 2011.

90. Tang YL, Zhao Q, Qin X, et al. Paracrine action enhances the effects of autologous mesenchymal stem cell transplantation on vascular regeneration in rat model of myocardial infarction. Ann Thorac Surg 2005;80:229-236; discussion 236-227.

91. Acharya C, Adesida A, Zajac P, et al. Enhanced chondrocyte proliferation and mesenchymal stromal cells chondrogenesis in coculture pellets mediate improved cartilage formation. J Cell Physiol 2012;227:88-97.

92. Wu L, Prins HJ, Helder M, et al. Trophic effects of mesenchymal stem cells in chondrocyte cocultures are independent of culture conditions and cell sources. Tissue Eng Part A 2012.

93. Gafni Y, Turgeman G, Liebergal M, et al. Stem cells as vehicles for orthopedic gene therapy. Gene Ther 2004;11:417-426.

94. Wu L, Wu Y, Lin Y, et al. Osteogenic differentiation of adipose derived stem cells promoted by overexpression of osterix. Mol Cell Biochem 2007;301:83-92.

95. Wu L, Zhu F, Wu Y, et al. Dentin sialophosphoprotein-promoted mineralization and expression of odontogenic genes in adipose-derived stromal cells. Cells Tissues Organs 2008;187:103-112.

96. Peng LH, Fung KP, Leung PC, et al. Genetically manipulated adult stem cells for wound healing. Drug Discov Today 2011;16:957-966.

97. Gimble JM, Grayson W, Guilak F, et al. Adipose tissue as a stem cell source for musculoskeletal regeneration. Front Biosci (Schol Ed) 2011;3:69-81.

98. Trippel SB, Ghivizzani SC, Nixon AJ. Gene-based approaches for the repair of articular cartilage. 
Gene Ther 2004;11:351-359.

99. Guo T, Zhao J, Chang J, et al. Porous chitosan-gelatin scaffold containing plasmid DNA encoding transforming growth factor-beta1 for chondrocytes proliferation. Biomaterials 2006;27:1095-1103.

100. Guo X, Zheng Q, Yang S, et al. Repair of full-thickness articular cartilage defects by cultured mesenchymal stem cells transfected with the transforming growth factor betal gene. Biomed Mater 2006;1:206-215.

101. Jin X, Sun Y, Zhang K, et al. Ectopic neocartilage formation from predifferentiated human adipose derived stem cells induced by adenoviral-mediated transfer of hTGF beta2. Biomaterials 2007;28:2994-3003.

102. Jin XB, Sun YS, Zhang K, et al. Tissue engineered cartilage from hTGF beta2 transduced human adipose derived stem cells seeded in PLGA/alginate compound in vitro and in vivo. J Biomed Mater Res A 2008;86:1077-1087.

103. Yang YR, Tian H. [Repair of articular cartilage defects by autologous adipose derived mesenchymal stem cell: experiment with rabbits]. Zhonghua Yi Xue Za Zhi 2008;88:2214-2218.

104. Kang Y, Liao WM, Yuan ZH, et al. In vitro and in vivo induction of bone formation based on adeno-associated virus-mediated BMP-7 gene therapy using human adipose-derived mesenchymal stem cells. Acta Pharmacol Sin 2007;28:839-849.

105. Kuroda R, Usas A, Kubo S, et al. Cartilage repair using bone morphogenetic protein 4 and musclederived stem cells. Arthritis Rheum 2006;54:433-442.

106. Matsumoto T, Cooper GM, Gharaibeh B, et al. Cartilage repair in a rat model of osteoarthritis through intraarticular transplantation of muscle-derived stem cells expressing bone morphogenetic protein 4 and soluble Flt-1. Arthritis Rheum 2009;60:1390-1405.

107. Lin L, Zhou C, Wei X, et al. Articular cartilage repair using dedifferentiated articular chondrocytes and bone morphogenetic protein 4 in a rabbit model of articular cartilage defects. Arthritis Rheum 2008;58:1067-1075.

108. Diekman BO, Estes BT, Guilak F. The effects of BMP6 overexpression on adipose stem cell chondrogenesis: Interactions with dexamethasone and exogenous growth factors. J Biomed Mater Res A 2010;93:994-1003.

109. Li J, Zhao Q, Wang E, et al. Dynamic compression of rabbit adipose-derived stem cells transfected with insulin-like growth factor 1 in chitosan/gelatin scaffolds induces chondrogenesis and matrix biosynthesis. J Cell Physiol 2012;227:2003-2012.

110. Lee SY, Lim J, Khang G, et al. Enhanced ex vivo expansion of human adipose tissue-derived mesenchymal stromal cells by fibroblast growth factor-2 and dexamethasone. Tissue Eng Part A 2009; 15:2491-2499.

111. Kilroy GE, Foster SJ, Wu X, et al. Cytokine profile of human adipose-derived stem cells: expression of angiogenic, hematopoietic, and pro-inflammatory factors. $\mathbf{J}$ Cell Physiol 2007;212:702-709.

112. de Crombrugghe B, Lefebvre V, Behringer RR, et al. Transcriptional mechanisms of chondrocyte differentiation. Matrix Biol 2000;19:389-394.

113. Lefebvre V, Huang W, Harley VR, et al. SOX9 is a potent activator of the chondrocyte-specific enhancer of the pro alpha1(II) collagen gene. Mol Cell Biol 1997;17:2336-2346.

114. Zhao Q, Eberspaecher H, Lefebvre V, et al. Parallel expression of Sox 9 and Col2a1 in cells undergoing chondrogenesis. Dev Dyn 1997;209:377-386.

115. Paul R, Haydon RC, Cheng H, et al. Potential use of Sox9 gene therapy for intervertebral degenerative disc disease. Spine (Phila Pa 1976) 2003;28:755-763.

116. Tsuchiya H, Kitoh H, Sugiura F, et al. Chondrogenesis enhanced by overexpression of sox 9 gene in mouse bone marrow-derived mesenchymal stem cells. Biochem Biophys Res Commun 2003;301:338-343.

117. Richardson SM, Curran JM, Chen R, et al. The differentiation of bone marrow mesenchymal stem cells into chondrocyte-like cells on poly-L-lactic acid (PLLA) scaffolds. Biomaterials 2006;27:4069-4078.

118. Yang Z, Huang CY, Candiotti KA, et al. Sox-9 facilitates differentiation of adipose tissue-derived stem cells into a chondrocyte-like phenotype in vitro. J Orthop Res 2011;29:1291-1297.

119. Han Y, Lefebvre V. L-Sox5 and Sox6 drive expression of the aggrecan gene in cartilage by securing binding of Sox9 to a far-upstream enhancer. Mol Cell Biol 2008;28:4999-5013.

120. Dy P, Smits P, Silvester A, et al. Synovial joint morphogenesis requires the chondrogenic action of Sox5 and Sox6 in growth plate and articular cartilage. Dev Biol 2010;341:346-359.

121. Yang HN, Park JS, Woo DG, et al. Chondrogenesis of mesenchymal stem cells and dedifferentiated 


\section{Chapter 2}

chondrocytes by transfection with SOX Trio genes. Biomaterials 2011;32:7695-7704.

122. Lee JM, Im GI. SOX trio-co-transduced adipose stem cells in fibrin gel to enhance cartilage repair and delay the progression of osteoarthritis in the rat. Biomaterials 2012;33:2016-2024.

123. Im GI, Kim HJ, Lee JH. Chondrogenesis of adipose stem cells in a porous PLGA scaffold impregnated with plasmid DNA containing SOX trio (SOX-5,-6 and -9) genes. Biomaterials 2011;32:4385-4392. 


\section{Chapter 3}

\section{Trophic effects of mesenchymal stem cells increase chondrocyte proliferation and matrix formation*}

Ling Wu, Jeroen Leijten, Nicole Georgi, Janine N. Post Clemens A. van Blitterswijk, Marcel Karperien

Department of Tissue Regeneration, MIRA-Institute for Biomedical Technology and Technical Medicine, University of Twente, Enschede, 7522NB, the Netherlands.

*Adapted from Wu L., et al., Tissue Eng Part A 17: 1425-36 


\begin{abstract}
Previous studies showed that co-culture of primary chondrocytes with various sources of multipotent cells results in a higher relative amount of cartilage matrix formation than cultures containing only chondrocytes. The aim of this study is to investigate the mechanism underlying this observation. We used co-culture pellet models of human mesenchymal stem cells (hMSCs) and human primary chondrocytes (hPCs) or bovine primary chondrocytes (bPCs) and studied the fate and the contribution to cartilage formation of the individual cell populations during co-culture. Enhanced cartilage matrix deposition was confirmed by histology and quantification of total glycosaminoglycan (GAG) deposition. Species specific quantitative PCR (qPCR) demonstrated that cartilage matrix gene expression was mainly from bovine origin when bPCs were used. Short tandem repeat (STR) analysis and species specific qPCR analysis of genomic DNA demonstrated the near complete loss of MSCs in co-culture pellets after 4 weeks of culture. In co-culture pellets of immortalized MSCs (iMSCs) and bPCs, chondrocyte proliferation was increased, which was partly mimicked using conditioned medium, and simultaneously preferential apoptosis of iMSCs was induced. Taken together, our data clearly demonstrate that in pellet co-cultures of MSCs and primary chondrocytes, the former cells disappear over time. Increased cartilage formation in these co-cultures is mainly due to a trophic role of the MSCs in stimulating chondrocyte proliferation and matrix deposition by chondrocytes rather than MSCs actively undergoing chondrogenic differentiation.
\end{abstract}




\section{Introduction}

Articular cartilage repair is a challenge due to the inability of cartilage to repair itself after damage. Autologous chondrocyte implantation (ACI) has become the golden standard treatment for large-size cartilage defects [1-2]. However, ACI creates donorsite injury and is dependent on two-dimensional expansion of isolated chondrocytes resulting in chondrocyte dedifferentiation [3].

To reduce the number of chondrocytes needed in ACI, a partial substitution of chondrocytes with pluripotent stem cells is a promising strategy. It has been reported that co-culture of bone marrow mesenchymal stem cells (MSCs) and articular chondrocytes enhanced matrix deposition [4-6] even in absence of the chondrogenic factors Transforming Growth Factor- $\beta$ (TGF- $\beta$ ) and dexamethasone (dex) [7]. Increased cartilage matrix formation was also found in co-culture of chondrocytes with other cell types, such as adipose-tissue derived stem cells, human embryonic stem cells and meniscus cells [8-11].

MSCs are promising for tissue repair because of their multi-lineage differentiation capacity [12]. Because of their importance in the development of articular cartilage, MSCs are a potential source for co-culture with chondrocytes. It is hypothesized that MSCs repair damaged tissue by differentiating into tissue specific cells replacing lost cells [13]. However, evidence suggests that differentiation into tissue specific cells cannot fully explain the benefits of transplanted MSCs in remodeling and recovery of damaged or lost tissue [14] [15-16]. These studies point to a central role of MSCs in tissue repair as trophic mediators, secreting factors promoting tissue specific cells to restore the damaged or lost tissue [17-18].

Two explanations have been proposed to explain increased cartilage formation in co-cultures of MSCs and articular chondrocytes. First, it has been suggested that increased cartilage formation in co-cultures is due to chondrogenic differentiation of MSCs stimulated by factors secreted by chondrocytes. Indeed, chondrocyte conditioned medium can induce chondrogenic differentiation of MSCs directly and in transwell cultures [19] [20]. However, it is unclear whether such an effect also occurs in cocultures in which the cells are in direct cell-cell contact. Second, studies have hypothesized that the increased cartilage matrix formation is due to stimulation of the 


\section{Chapter 3}

chondrocytes by MSCs [6]. Scientific evidence for this hypothesis is rather limited due to the inability to distinguish between the contributions of the individual cell populations to cartilage formation.

In this study we have addressed these issues by setting up pellet co-culture models of human MSCs (hMSCs) and either human (hPCs) or bovine primary chondrocytes (bPCs). Using a xenogenic system allowed us to determine the contribution of each cell population to the increased cartilage formation by using species specific gene expression analysis, whereas xenogenic specific effects were excluded in the human co-culture system. We examined chondrogenic gene expression, cell apoptosis and cell proliferation in human and bovine cell populations. Our data clearly demonstrates that the increased cartilage deposition in co-cultures is mainly due to a trophic role of the MSCs in stimulating chondrocyte proliferation and matrix deposition rather than MSCs actively undergoing chondrogenic differentiation.

\section{Materials and Methods}

\section{Cell culture and expansion}

Bovine primary chondrocytes (bPCs) were isolated from full-thickness cartilage knee biopsies of female calves of approximately 6 months old. Cartilage was separated and digested as previously described [21]. Human primary chondrocytes (hPCs) were obtained from full thickness cartilage dissected from knee biopsies of a patient undergoing total knee replacement as published previously [11]. Mesenchymal stem cells were isolated from aspirates as described previously [22]. The use of bone marrow aspirates and human knee biopsies was approved by a local Medical Ethical Committee. Donor information of human primary cells is listed in Supplementary Table S1. We define the "primary" cells (bPCs, hPCs and hMSCs) in this manuscript as cells with low passage number without immortalization. iMSCs were kindly provided by Dr. O. Myklebost (Oslo University Hospital, Norway). Culture condition and characteristics of iMSCs are described in Supplementary figure S1.

To form high density micro mass cell pellets, 200,000 cells per well were seeded in a round bottom 96 wells plate in chondrocyte proliferation medium (DMEM supplemented with $10 \%$ FBS, $1 \times$ non-essential amino acids, $0.2 \mathrm{mM}$ ascorbic acid 2phosphate (AsAP), $0.4 \mathrm{mM}$ proline, $100 \mathrm{U}$ penicillin $/ \mathrm{ml}$ and $100 \mu \mathrm{g} / \mathrm{ml}$ streptomycin) or 
chondrogenic differentiation medium (see Supplementary figure S1) and centrifuged for $3 \mathrm{~min}$ at 2000rpm. Medium was refreshed twice a week. For co-cultures, iMSCs or hMSCs and bPCs or hPCs were mixed at ratios of $80 / 20 \%$ and $50 / 50 \%$.

All reagents used for were purchased from Invitrogen (Paisley, UK) unless otherwise stated. Common chemicals were purchased from Sigma-Aldrich (Germany).

\section{Histology}

Cell pellets were fixed with $10 \%$ formalin for 15 minutes and embedded in Paraffin using routine procedures. Sections of $5 \mu \mathrm{m}$ were cut and stained for sulfated glycosaminoglycans (GAG) with alcian blue combined with counterstaining of nuclear fast red to visualize nuclei, or stained with toluidine blue alone.

\section{Quantitative GAG and DNA assay}

Cell pellets $(\mathrm{N}=6)$ were washed with $\mathrm{PBS}$ and frozen overnight at $-80{ }^{\circ} \mathrm{C}$. Subsequently, they were digested and measured for GAG quantification as previously reported [11]. Relative cell number was determined by quantification of total DNA using a CyQuant DNA Kit, according to the manufacturer's instructions.

\section{DNA isolation, RNA isolation and quantitative PCR}

Total DNA was isolated from pellet cultures with the QIAamp DNA Mini Kit (Qiagen) according to the manufacturer's protocol. Total RNA was isolated from pellet culture with the RNeasy Mini Kit (Qiagen). One microgram of total RNA was reversetranscribed into cDNA using the iScript cDNA Synthesis kit (Bio-Rad). The primers for qPCR are listed in supplementary table S2 and S3. Detailed description for qPCR can be found in the Supplementary Methods.

\section{Cell tracking with organic fluorescent dyes}

The organic fluorescent dyes, CM-DiI and CFSE were used for cell tracking in co-cultures. Cells were labeled according to the manufacturer's protocols.

\section{EdU and TUNEL staining}

For labeling of newly synthesized DNA, EdU (5-ethynyl-2'-deoxyuridine) was added to the culture media at a concentration of $10 \mu \mathrm{M}, 24$ hours before harvesting the samples. Cell pellets were then washed with PBS and fixed with $10 \%$ formalin for 15 min. Samples were embedded in cryomatrix, and cut into $10 \mu \mathrm{M}$ sections with a cryotome (Shandon). Sections were permeabilized and stained for EdU with Click-iT ${ }^{\circledR}$ 
EdU Imaging Kit. Cryosections were also stained for DNA fragments with DeadEnd Fluorometric TUNEL System (Promega). Nuclei were counterstained with Hoechst 33342.

\section{Image acquisition and analysis}

All fluorescent images were taken with a BD pathway 435 confocal microscope (BD Biosciences), unless otherwise stated. Using montage capture, images of high resolutions were obtained covering the entire section of a pellet. Separate images were captured using BP536/40 (Alexa 488), BP593/40 (DiI) and LP435 (Hoechst 33342) and pseudocolored green, red and blue respectively. ImageJ software [23] was used for cell counting. Briefly, we manually set a threshold to avoid artifacts. The number of green cells, red cells, green + red cells and total cells were counted by running plug-ins written in macro language of ImageJ (available on request). Values represent the mean $+/$ - standard error of at least 3 biological replicates.

\section{Preparation of conditioned medium}

For conditioned medium, DMEM was incubated with iMSCs of $90 \%$ confluence for $48 \mathrm{~h}$, passed through a $0.22 \mathrm{~mm}$ filter, and stored at $-20{ }^{\circ} \mathrm{C}$. Upon usage, conditioned medium was thawed, transferred to Amicon Ultra-15 Centrifugal Filter Unites (Millipore, Billerica, MA) with a cut off of 3000D Nominal Molecular Weight Limit, and centrifuged at $4000 \times \mathrm{g}$ for 40 minutes. The concentrated solute (still named conditioned medium) was supplemented with all chemicals needed for chondrocyte proliferation medium (see cell culture and expansion).

\section{Short Tandem Repeats (STR) analysis}

Genomic DNA samples were extracted from pellets with the QIAamp DNA Mini Kit (Qiagen). The sixteen loci of the kit PowerPlex 16 System (Promega) were amplified, typed, sequenced and analyzed by ServiceXS B.V. (Leiden, the Netherlands). Specific alleles for the donor of hMSCs and the donor of hPCs were found in six loci (D7S820, D5S818, D13S317, D16S539, CSF1PO and Penta_D). These alleles were used to define the origin of cells in allogeneic co-culture of hMSCs and hPCs. The amount of DNA present for each donor was calculated from the areas of the electropherogram for each locus of hMSCs' or hPCs' specific alleles and the ratio of hMSCs and hPCs was determined. 


\section{Statistical analysis}

For the experiments using primary human cells (hMSCs and hPCs), three donors were tested, which showed similar results. Each experiment was performed at least in triplicate. So, only data from one representative donor is shown. Experiments using iMSCs and bPCs were performed at least in triplicate with similar data. A representative experiment is shown. Differences between different ratios of co-cultures of MSCs and primary chondrocytes were examined for statistical significance with oneway analysis of variance (ANOVA) followed by Tukey HSD Tests. Comparisons between iMSCs and bPCs in the same conditions were made using the Student's test. $P$ values of $<0.05$ were considered significant.

\section{Results}

\section{Co-culturing hMSCs with hPCs enhanced cartilage matrix formation}

In order to study the contribution of MSC and chondrocytes on cartilage matrix formation we co-cultured human MSCs (hMSC) with human primary chondrocytes (hPCs) from different donors. After 4 weeks co-culture in chondrogenic differentiation medium, histology (Figure 1A) and GAG assay (Figure 1B) indicated that co-culture of hMSCs and hPCs increased cartilage formation. To determine the ratio of MSC and PC after prolonged co-culture we isolated genomic DNA, and STR loci with different repeat sizes in the different donors were analyzed. The results of locus D7S820 (Figure 1C) as well as analysis of other 5 STR loci (Table S4), indicated that the proportion of hMSCs decreased significantly.

In order to elucidate the mechanisms behind the apparent loss of MSC in our co-culture system, we used xenogenic co-cultures of hMSCs and bPCs to enable identification of the role of each of the cell types in co-culture in pellet cultures. An advantage of these xenogenic co-cultures is that this system is more stable than coculture systems that depend on donor hPCs isolated after total knee replacement surgery.

\section{Xenogenic co-culture of hMSC and bPCs show enhanced chondroinduction}

To allow long term cell tracking in co-cultures, we setup a pellet co-culture model of hMSCs and bPCs. Cells were mixed in different ratios and pellet culture was performed in chondrocyte proliferation medium lacking TGF- $\beta$ and dexamethason. After 4 weeks, histology and GAG quantification were performed to evaluate cartilage 


\section{Chapter 3}
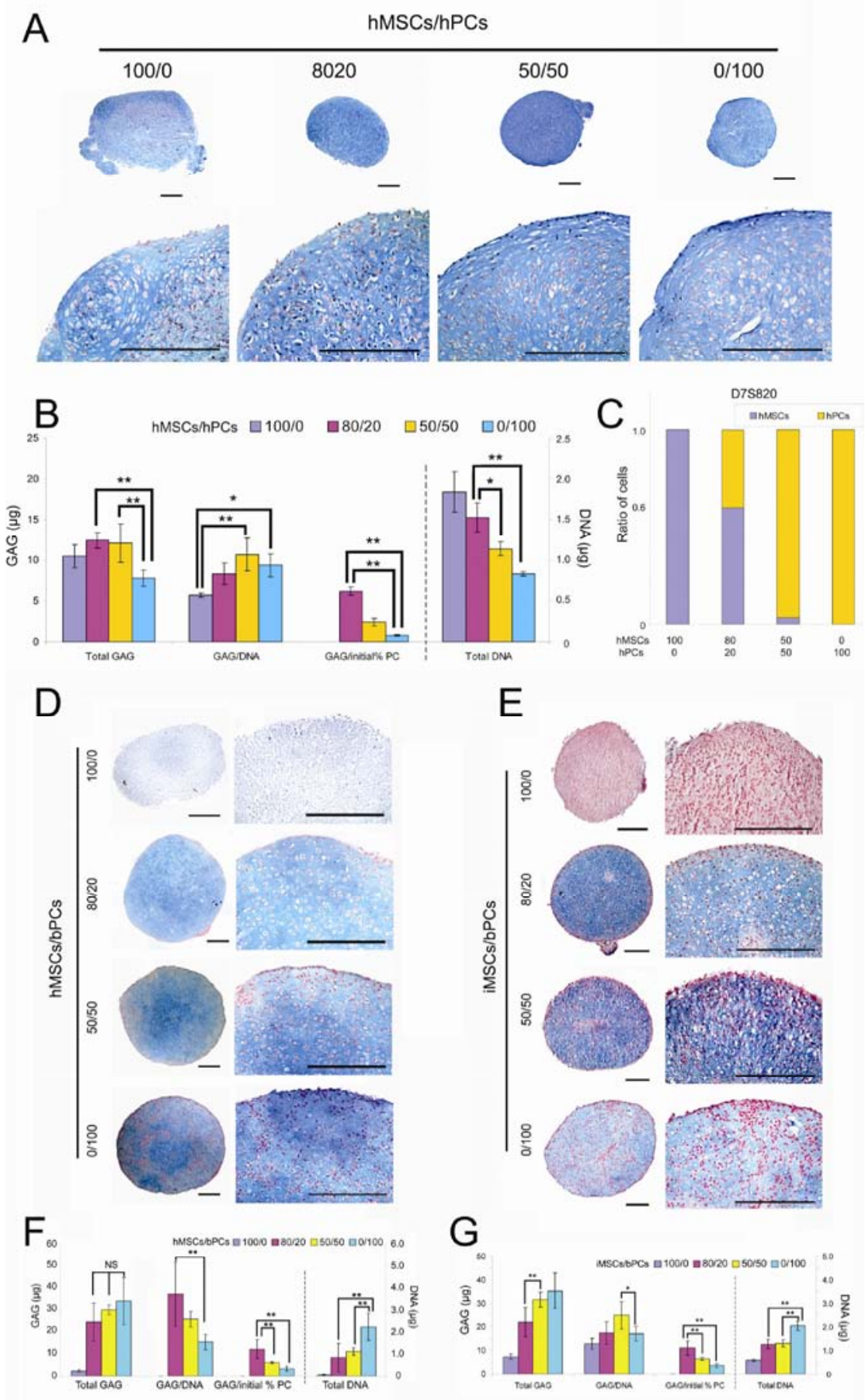
Figure 1 hMSCs / hPCs co-cultures enhance cartilage matrix formation and show decrease of MSCs after 4 weeks of culture; and co-culture of mesenchymal stem cells and chondrocytes increases cartilage matrix formation. (A) Alcian blue staining indicates the presence of GAG. Pellets were cultured in chondrogenic differentiation medium (as described in supplementary materials) for 4 weeks before examination. Scale bar $=200 \mu \mathrm{m}$. (B) Biochemical assay shows an increase in GAG in co-culture pellets. Amount of GAG and DNA of pellets $(\mathrm{N}=6)$ were measured 4 weeks after culture in chondrogenic differentiation medium. Ratios of hMSCs and bPCs are indicated by different colors of bars. Scale on the left is for "Total GAG", "GAG/DNA" and "GAG/initial 10\%PC", while scale on the right is for "Total DNA". Asterisk represents $P<0.05$. Double asterisk represents $P<0.01$. NS=Not Significant. Error bar reflects Standard Deviation (S. D.). (C) Analysis of STR locus D7S820 reflects ratios of hMSCs and hPCs after 4 weeks coculture. Initial ratio of hMSCs and hPCs are indicated at the bottom of the bar chart. (D) Alcian blue staining shows the presence of GAG in pellets cultured in chondrocytes proliferation medium. Ratios of hMSCs and bPCs are indicated on the left of the images. The left panel shows overviews of pellets, while the right panel shows magnified pictures. Scale bar=200 $\mu \mathrm{m}$. (E) Alcian blue staining of pellets cultured in chondrocytes proliferation medium. Ratio of iMSCs and bPCs is indicated on the left of the images. The left panel shows overviews of pellets, while the right panel shows magnified pictures. Scale bar $=200 \mu \mathrm{m}$. (F) Biochemical assay shows an increase in GAG in co-culture pellets. Amount of GAG and DNA of pellets $(\mathrm{N}=6)$ was measured 4 weeks after culture in chondrocyte proliferation medium. Ratios of hMSCs and bPCs are indicated by different colors of bars. Scale on the left is for "Total GAG", "GAG/DNA" and "GAG/initial 10\%PC", while scale on the right is for "Total DNA". Asterisk represents $P<0.05$. Double asterisk represents $P<0.01$. NS=Not Significant. Error bar reflects Standard Deviation (S. D.). (G) Biochemical assay of pellets $(\mathrm{N}=6)$ at 4 weeks after culture in chondrocyte proliferation medium. Ratios of iMSCs and bPCs are indicated by different colors of bars. Scale on the left is for "Total GAG", "GAG/DNA" and "GAG/initial 10\%PC", while scale on the right is for "Total DNA".Asterisk represents $P<0.05$. Double asterisk represents $P<0.01$. NS=Not Significant. Error bar reflects Standard Deviation (S. D.).

formation. Alcian blue staining indicated the presence of GAG in all experimental groups except in the 100\% hMSCs pellets (Fig. 1D left panel) in concordance with the absence of chondrogenic factors in the medium. In the positively stained areas at higher magnification (Fig. 1D right panel), cells showed a typical chondrocyte morphology and embedding in lacunae. Similar data were obtained by toluidine blue staining (supplementary figure S2). GAG quantification showed a trend of decreased total GAG with increasing seeding percentage of hMSCs (Fig. 1F). However, when total GAG content was normalized to DNA or to the initial seeding percentage of bPCs, co-culture pellets showed significantly higher GAG content. Similar data were obtained when 
different MSC donors were used (data not shown).

To avoid the effects of donor variation of primary cultured MSCs [24], we replaced hMSCs with a telomerase immortalized hMSC cell line (iMSCs). This cell line resembled primary cultured MSCs in their ability to differentiate into the adipogenic, osteogenic and chondrogenic lineages (supplementary figure S1), but had relatively low capacity of chondrogenic differentiation As shown in Figure 1E and G, co-culture of bPCs with iMSCs for 4 weeks increased cartilage formation after correction for DNA content or initial seeding percentage of bPCs as compared to hMSCs. Despite the relatively low chondrogenic potential of iMSCs, increased cartilage matrix formation was observed in co-culture of bPCs and iMSCs. This demonstrated that iMSCs show comparable behavior to hMSCs in co-cultures with regard to enhanced cartilage formation, indicating that it is not the chondrogenic capacity of the MSCs that is responsible for enhanced chondroinduction.

\section{Chondrocytes are located at the periphery of the cell pellet}

We used organic fluorescent dyes to label individual cell populations in pellet co-cultures for short term cell tracking. Pellets were formed after 1 day of culture (Figure 2A). Rather than forming a homogenous pellet, both cell populations tended to segregate. This process continued in the following days and the dynamic cell pellets became more and more stable. After 4 days of co-culture, pellets were organized in a layer-like structure in which iMSCs resided predominantly in the core of the pellet and the bPCs, mixed with a sub-fraction of iMSCs, were predominantly found at the periphery. These observations are in agreement with the "differential adhesion hypothesis" which stipulates that mixed heterotypic cells rearrange to adopt a combination-specific anatomy [25]. From day 5 onwards, fluorescent dye transfer between labeled and non-labeled cells in the pellets became apparent as reported previously [26]. This made it impossible to perform long-term cell tracking in coculture pellets using CM-DiI and/or CFSE labeling of cell populations.

\section{Enhanced cartilage matrix formation originates from bPCs.}

After 1 day and 4 weeks of culture we isolated genomic DNA from the cell pellets and performed species specific qPCR for genomic GAPDH. As shown in figure $2 \mathrm{~B}$, after 1 day the ratio of genomic human and bovine DNA was in line with the 
seeding percentage of both cell populations. The percentage of human DNA was slightly higher which is most likely explained by faster aggregation of the iMSCs in pellets. Remarkably, after 4 weeks of culture, the co-culture pellets contained predominantly DNA of bovine origin indicative for an overgrowth of bovine cells or a loss of human cells during the 4 week cell culture period. DNA analysis of co-culture pellets at 1, 2 and 3 weeks of culture demonstrated a steep drop in human DNA between 1 and 2 weeks with a further gradual decline at weeks 3 and 4 (data not shown).

An even more striking difference was found in mRNA isolated at 4 weeks. GAPDH mRNA in the co-culture pellets was from bovine origin (Figure 2C). Even in cell pellets with an initial seeding of $80 \%$ iMSCs, hardly any human mRNA was detected. Similar data were found in co-culture pellets of hMSCs and bovine chondrocytes after 4 weeks of culture also demonstrating the near absence of human DNA in the cell pellets, which is fully in line with the data obtained in co-culture pellets of iMSCs and bPCs (Figure 2D) and hMSC and hPC (Figure 1C).

We next performed species specific qPCR to study the origin of the mRNA expression of chondrogenic genes in co-culture pellets (Figure 2E-G). At week 4, only expression of chondrogenic genes from bovine origin were detected in co-culture pellets. This data suggested that the cartilaginous matrix in co-culture pellets is from bovine origin. This observation, combined with the observation that in allogeneic co-cultures the percentage of MSCs decreased during prolonged culturing, suggests that the enhanced contribution of chondrocytes in the matrix formation may be due to PCs proliferation or MSC cell death.

\section{iMSCs co-cultured with bPCs die via apoptosis}

To determine whether MSC undergo apoptosis during prolonged cell culture we performed a fluorescent TUNEL assay. At week 1 and 2, high numbers of TUNEL positive cells were found in all cell pellets containing iMSCs, but not in pure bPCs cell pellets (Figure $2 \mathrm{H}$ and I). TUNEL positive cells were predominantly present in the periphery of the pellets which is mostly composed of bPCs mixed with iMSCs (Figure 2A). Fewer TUNEL positive cells were found in the core of the pellet. Cell death in iMSC containing pellets started to increase significantly from day 5 onwards. From this time point cell tracking results by fluorescent labeling of cell populations became 

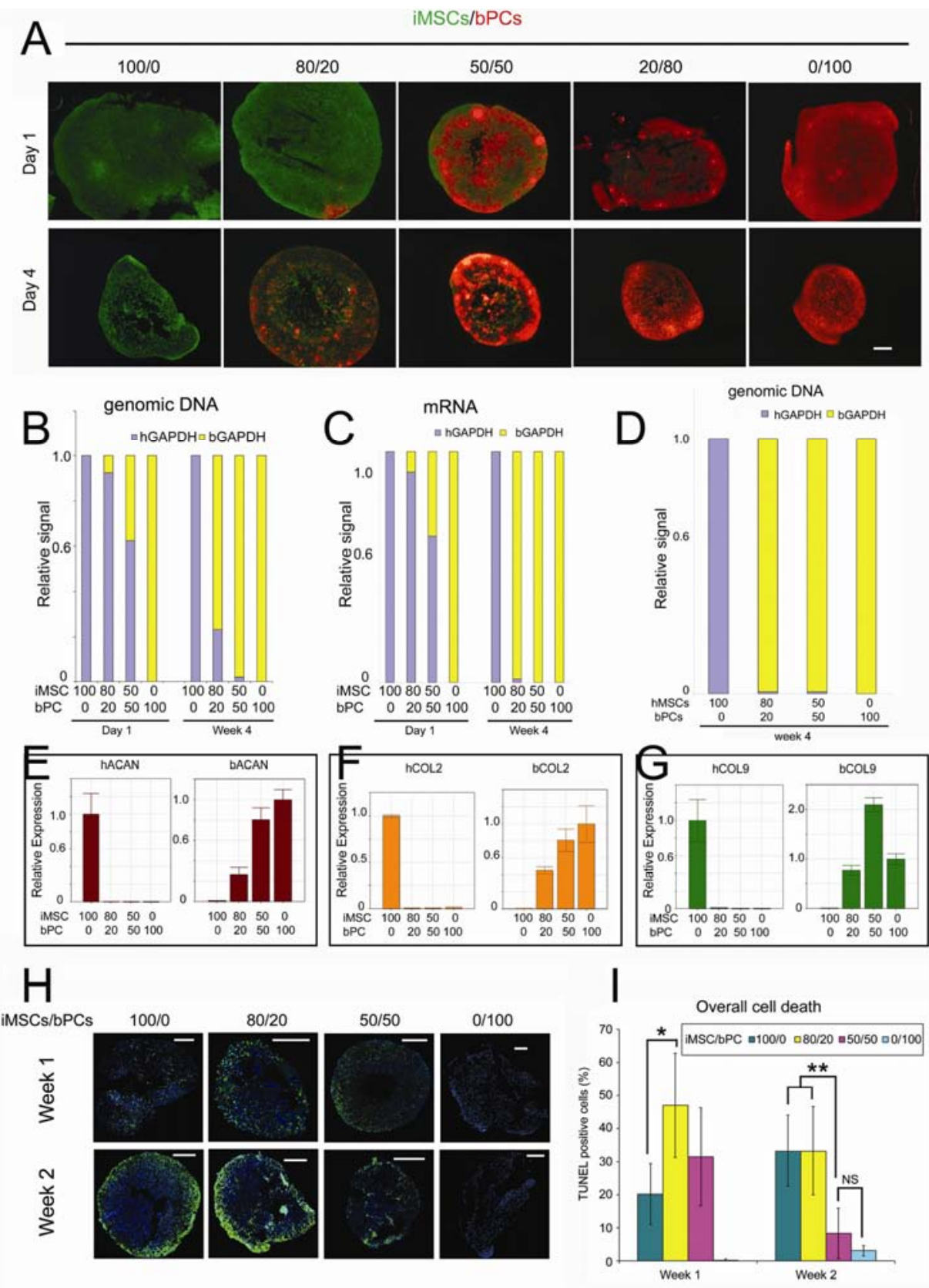
Figure 2 Cartilage matrix is from bovine origin; and preferential cell deaths of MSCs by apoptosis. (A) Cell assembly of iMSCs and bPCs in co-culture pellets. iMSCs and bPCs were labeled with CFSE O (green) and CM-DiI (red) respectively, mixed at different ratios and then cultured in chondrocyte proliferation medium. At day 1 and day 4, pellets were harvested for cryosection. Images were made directly on the sections without any treatment. Scale bar=200 $\mu$ m. (B) Species specific qPCR of GAPDH in co-cultures of iMSCs and bPCs at genomic DNA level. Genomic DNA was extracted from pellets $(\mathrm{N}=3)$ at day 1 and week 4. (C) Species specific qPCR of GAPDH in co-cultures of iMSCs and bPCs at mRNA level. RNA was extracted from pellets at day 1 and week 4. (D) Species specific qPCR of GAPDH in co-cultures of hMSCs and bPCs at genomic DNA level. Genomic DNA was extracted from pellets $(\mathrm{N}=3)$ at week 4. (E-G) Expression levels of ACAN (E), COL2 (F) and COL9 (G) were examined by species specific qPCR. RNA samples were extracted from pellets $(\mathrm{N}=3)$ cultured in chondrocyte proliferation medium for 4 weeks. Relative expression levels were obtained by normalization of human or bovine specific signals to cross species-specific GAPDH and $\beta$-actin signals. For human specific genes, values are relative amounts to 100/0 iMSC/bPC group. For bovine specific genes, values are relative amounts to 0/100 iMSC/bPC group. Error bar reflects Standard Deviation (S. D.). (H) TUNEL staining of pellets. Cell pellets were cultured in chondrocyte proliferation medium for 1 week or 2 weeks before harvesting for cryosection. TUNEL positive cells were visualized with fluorescent labeling (green). Nuclei were counterstained with Hoechst 33342 (blue). Scale bar=200 $\mu$ m. (I) Quantification of TUNEL positive cells. Ratios of iMSCs and bPCs are indicated by bar colors. Data from 3 pellets were analyzed for statistic significance. Asterisk represents $P<0.05$. Double asterisk represents $P<0.01$. NS=Not Significant. Error bar reflects Standard Deviation (S. D.).

unreliable due to non-specific dye transfer. Since the TUNEL positive cells are predominantly found in iMSCs containing cell pellets and human DNA over time disappears from the cell pellets we concluded that cell death by apoptosis at least partially explains the disappearance of human DNA from co-culture cell pellets.

\section{iMSCs stimulate chondrocyte proliferation in pellet co-cultures}

We then examined cell proliferation in co-culture pellets using EdU incorporation. We focused on time points up to 3 days, in which organic fluorescent dyes are highly reliable for cell tracking [27]. bPCs were labeled with CM-DiI (red) to distinguish them from iMSCs. At day 1, EdU positive cells were evenly distributed over the pellet. At day 2 and day 3, EdU positive cells were predominantly found at the periphery of the pellets where red labeled bPCs resided (Fig. 3A). We determined the percentage of EdU positive iMSCs or bPCs in co-cultures. Generally, co-culture increased the proliferation of both iMSCs and bPCs (Figure 3B and 3C). Interestingly, 
Chapter 3

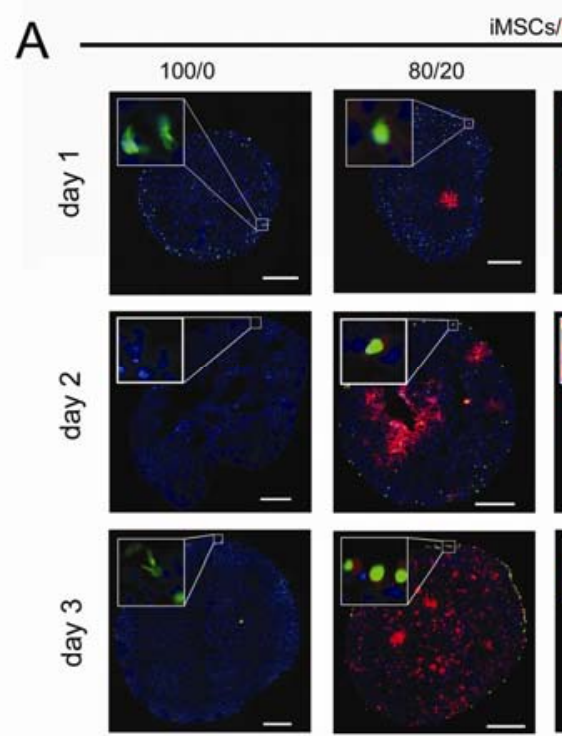

B Proliferation rate of iMSCs
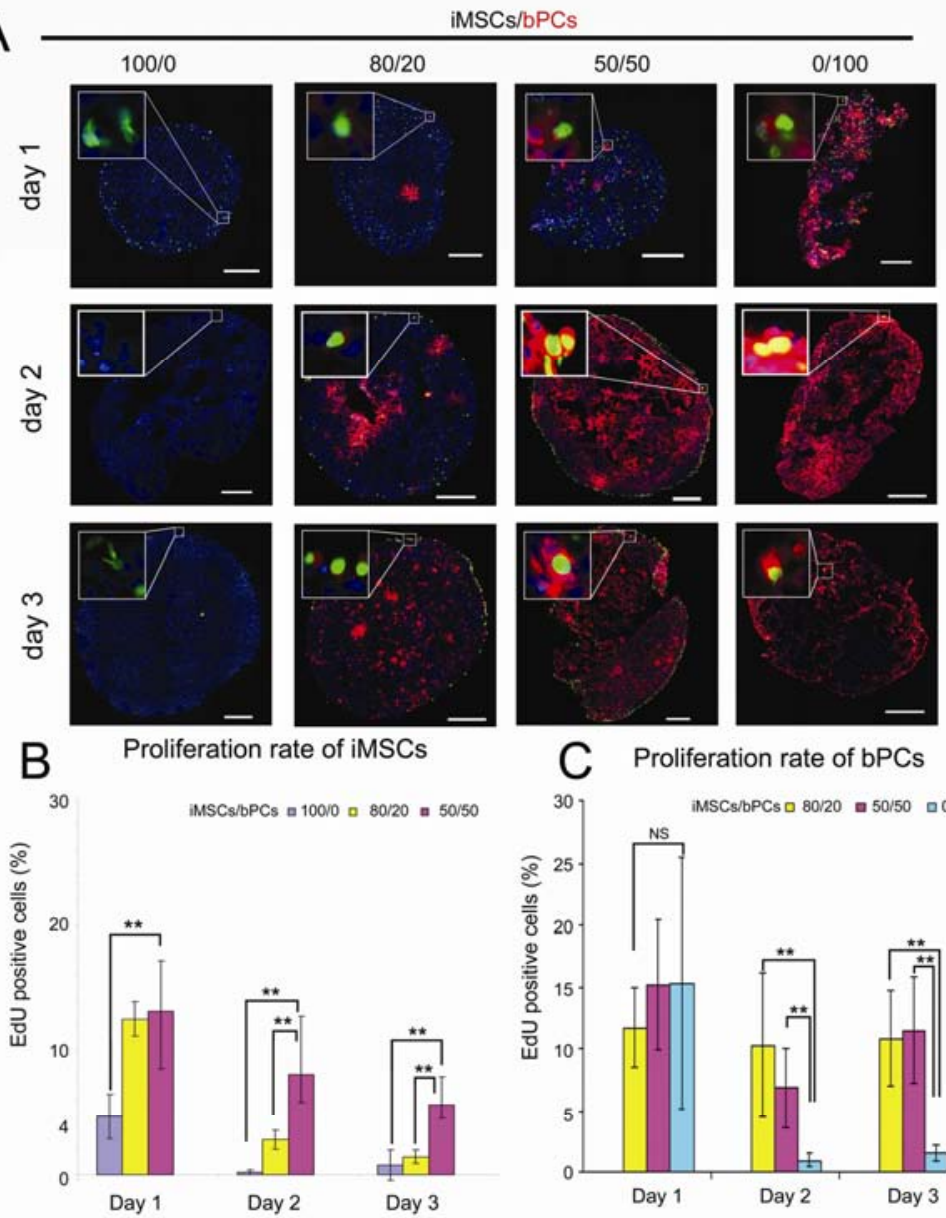

Droliferation Medium
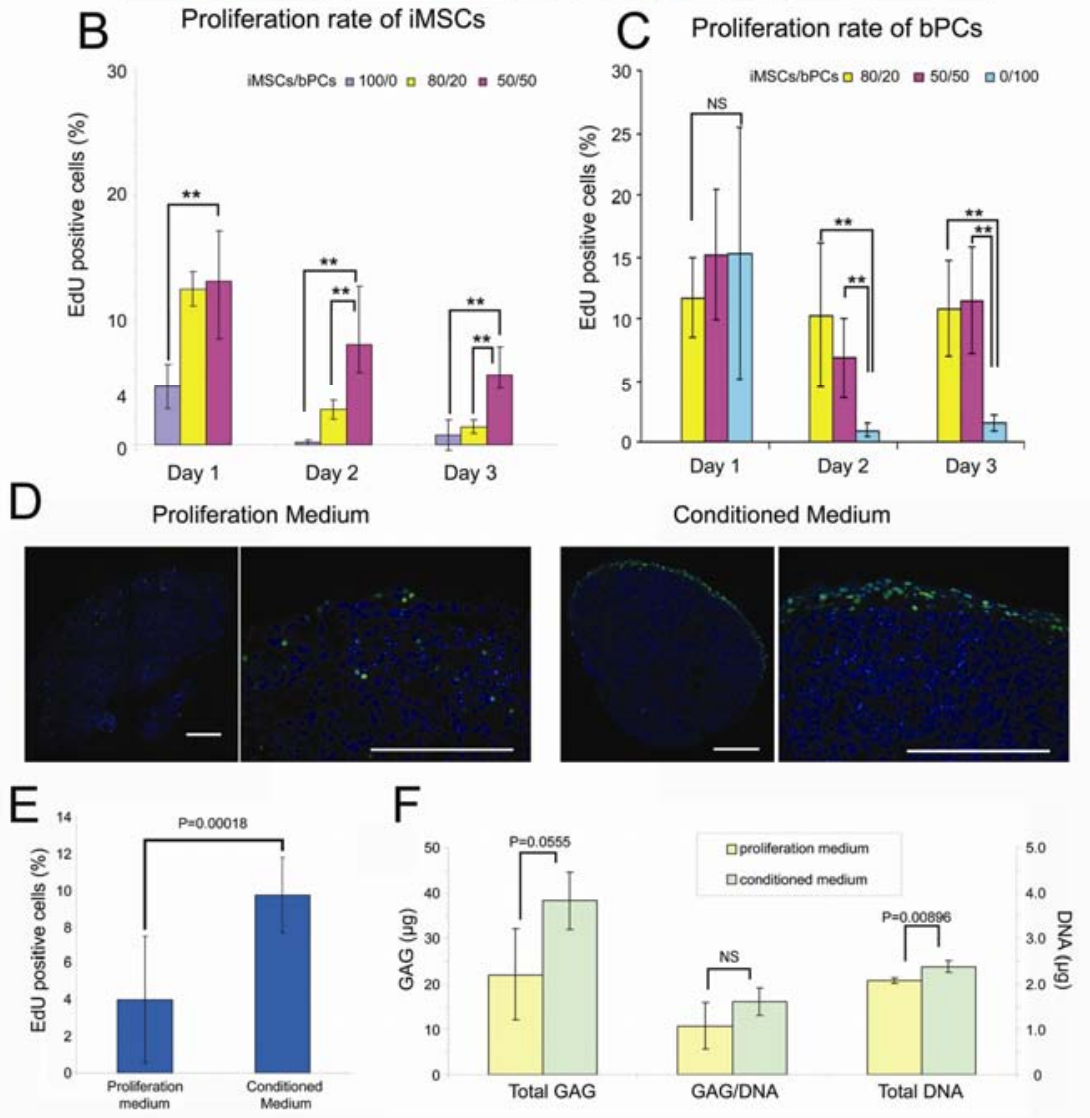
Figure 3 MSCs stimulate chondrocyte proliferation in co-culture pellets; and conditioned medium of iMSCs enhances proliferation of chondrocytes. (A) EdU staining of pellets at day 1, day 2 and day 3. bPCs were labeled with CM-DiI (red). EdU incorporation into newly synthesized DNA was visualized by Alexa 488 (green). Nuclei were counterstained with Hoechst 33342 (blue). Scale bar=200 $\mu$ m. (B) Quantification of EdU positive iMSCs in all conditions. The initial ratios of MSCs and bPCs are indicated by bar colors. Asterisk represents $P<0.05$. Data from 3 pellets were analyzed for statistic significance. Double asterisk represents $P<0.01$. NS=Not Significant. Error bar reflects Standard Deviation (S. D.). (C) Quantification of EdU positive bPCs in all conditions. The initial ratios of MSCs and bPCs are indicated by bar colors. Data from 3 pellets were analyzed for statistic significance. Asterisk represents $P<0.05$. Double asterisk represents $P<0.01$. NS=Not Significant. Error bar reflects Standard Deviation (S. D.). (D) EdU staining of bPCs pellets at day 2 after culturing in chondrocyte proliferation medium or conditioned medium of iMSCs. EdU incorporation into newly synthesized DNA was visualized by Alexa 488 (green). Nuclei were counterstained

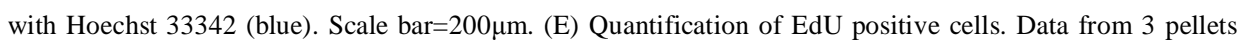
were analyzed for statistic significance. $P$-value indicated in the bar chart is calculated by student's $t$-test. (F) GAG and DNA assay were performed at week 1 after culturing in chondrocyte proliferation medium or conditioned medium of iMSCs. The left scale is for "Total GAG" and "GAG/DNA", while the right scale is for "Total DNA". P-values indicate on the graph were calculated with the Student's $t$-test. NS=Not Significant. Error bar reflects Standard Deviation (S. D.).

the percentage of EdU positive bPCs was higher than that of iMSCs in co-cultures of $80 \%$ iMSCs and 20\% bPCs starting from day 2 onwards (Figure 3B and C).

Similar data were obtained in dye swap experiments in which iMSCs instead of bPCs were labeled with CM-DiI demonstrating that enhanced proliferation of bPCs in co-culture pellets was not an artifact of cell labeling (Supplementary figure S3). These data show that the change in ratio between MSC and PC during prolonged co-culturing is in addition to apoptosis also due to increased proliferation of chondrocytes in pellet cultures.

\section{iMSC conditioned medium increases bPCs proliferation and matrix formation}

To examine the effects of secreted factors, we compared proliferation and matrix formation of bPCs when cultured in proliferation medium or in 50 100 times concentrated iMSC conditioned medium. The concentrate was dissolved in chondrocyte proliferation medium. Pellets of bPCs cultured for 1 week in iMSCs conditioned proliferation medium showed higher EdU incorporation than cells cultured in nonconditioned proliferation medium (Figure 3D and E). Like in co-culture pellets EdU positive cells were predominantly found in the periphery of the pellet. Higher EdU 


\section{Chapter 3}

incorporation was associated with increased DNA content. Additionally, total GAG content showed an increase, but GAG corrected for DNA was not significantly different between the two conditions (Figure 3F).

\section{Discussion}

It has been shown that conditioned medium of chondrocytes induced osteochondrogenic differentiation of MSCs [19] and co-culture of MSCs and chondrocytes in 3-dimensional environments induced chondrogenic gene expression in MSCs [28]. Based on these studies, it was suggested that the beneficial effects of co-culturing chondrocytes and MSCs in cartilage matrix formation are largely due to the differentiation of MSCs into chondrocytes. In this study, we show that pellet co-cultures of chondrocytes and MSCs in chondrocyte proliferation medium benefit cartilage formation. Furthermore, we observe a significant decrease in MSCs caused by a preferential cell death of MSC. After 4-weeks of culture this results in an almost homogeneous cartilage construct, in which mainly chondrocyte-derived cells reside. The beneficial effects of the pellet co-culture are largely due to stimulation of proliferation and matrix formation of chondrocytes induced by a trophic effect of the MSCs. Our investigation distinguishes itself from comparable studies, the design of which did not allow discrimination between the contributions of individual cell populations to cartilage matrix formation [5-6]. Although we cannot completely rule out the possibility that a subset of MSCs differentiated into chondrocytes and directly participated in cartilage formation, our data suggests that this may apply to a minority of cells only.

In pellet co-cultures of hMSCs and bovine chondrocytes, one may argue that our observations are due to a species difference which may hamper the response of bovine chondrocytes to human MSCs and vice-versa. However, species specificity cannot explain our findings since similar observations were made in a fully human coculture model, indicating that in both models comparable mechanisms are likely operational. In addition, we show that the mechanisms underlying these observations are not donor specific, but are due to cell type specific contribution of MSCs as well as the chondrocytes. As shown in this study, as well as in many other studies [5-6, 11], co- 
culture of hMSCs or other cell types[29-30] with xenogenic chondrocytes appears a good model to study cell specific contributions to tissue formation.

In our studies, we have used hTERT immortalized hMSCs[31] as well as primary hMSCs. The iMSCs used in this study had a reduced chondrogenic potential. This lack of chondrogenic capacity did not impair their ability to stimulate cartilage formation in pellet co-cultures, furthermore providing evidence that chondrogenic differentiation of MSC does not significantly contribute significantly to the enhanced cartilage formation. Furthermore, similar results were obtained with primary hMSCs. Our data do indicate that chondrogenic capacity of cells used in co-cultures is not essential for stimulation of cartilage formation by chondrocytes in line with previous observations [11]. In addition, our data suggests that the relatively old age (60+ years) of the MSC donors does not affect their ability to simulate cartilage formation in coculture.

Cell proliferation in pellet co-cultures was studied using EdU incorporation in DNA of proliferating cells. Cell proliferation was significantly increased in co-culture pellets compared to pellets of pure cell populations. By using cell specific labeling techniques and dye swap experiments, it was shown that EdU was preferentially incorporated in chondrocytes, which reside predominantly in the periphery of the cell pellet. This suggests that the MSCs are potent stimulators of chondrocyte proliferation in pellet co-cultures. Limited proliferation of cells was found in the core of the cell pellet in which predominantly MSCs resided. Since EdU is extremely small (252 Dalton), this molecule is likely to penetrate with high efficiency in the pellet [32], suggesting that the preferential EdU labeling of cells in the periphery of the pellets is not an artifact caused by diffusion limitation. It is assumed that absence of proliferating MSCs in the center of the pellets is likely due to space limitation in the compacted core creating an environment which is not permissive for cell division [33-34].

In co-culture pellets significant numbers of TUNEL positive MSCs were observed after 1 and 2 weeks of culture suggesting that MSCs most likely died via apoptosis. Also in pellets composed of $100 \%$ MSCs but not $100 \%$ bPCs, significant TUNEL staining was observed. Cell labeling experiments in pellet co-cultures demonstrated that the majority of the TUNEL positive cells were hMSCs. This is in line 


\section{Chapter 3}

with the STR and genomic DNA analysis at the end of the culture period showing the disappearance of the MSCs from the co-cultures over-time. Our data suggest that the disappearance of MSCs in pellet co-cultures is most likely caused by apoptosis. Interestingly, TUNEL positive cells were predominantly found in the periphery of the pellet in which MSCs co-resided with chondrocytes. TUNEL positivity was higher in co-culture pellets compared to pellets of pure cell populations. This suggested that in addition to suboptimal culture conditions of MSCs in pellets, the presence of chondrocytes may have contributed to the death of MSCs. This may be caused by secreting apoptosis-inducing cytokines [35]. Furthermore, changes in extracellular matrix (ECM) in pellet cultures as compared to natural ECM of MSCs may influence the fate of MSCs [36-38] and this may have contributed to the increased cell death. Other explanations for death of MSCs in pellets could be cell compaction, and nutrition or space limitation in pellets [39-40]. However, the relatively low levels of TUNEL positive cells in the core of the pellet compared to the periphery suggests that nutrient or oxygen limitation, which are likely most pronounced in the core of the pellet, are insufficient to induce cell death.

We provide evidence that the induction of chondrocyte proliferation by MSCs is most likely caused by (a) secreted factor(s), since this effect was at least partly mimicked by using MSC conditioned medium. It has been reported that MSCs secrete a broad range of growth factors and cytokines including interleukin-6 (IL-6), hepatocyte growth factor (HGF), and vascular endothelial growth factor (VEGF), which enhance cell viability and proliferation in vitro and restore functions of damaged tissue in vivo [41-42]. IL-6, for example, has been described to induce cartilage repair by increasing chondrocyte proliferation and stimulation of expression of cartilage matrix proteins and BMP-7 [43]. On the other hand (a) secreted factor(s) by MSCs cannot fully explain increased cartilage formation in co-culture pellets, since the relative deposition of glycosaminoglycans per DNA was not significantly different between pellets cultured in proliferation medium and that in conditioned medium. This indicates that conditioned medium only stimulated chondrocyte proliferation but not relative GAG amount per DNA, such as observed in co-culture pellets. This is in line with other reports demonstrating a role of cell-cell contact in cartilage formation improvement in co- 
cultures [44-46]. Therefore, it is likely that in addition to the trophic effects of MSCs mediated by secreted factors, enhanced cartilage formation in co-culture with chondrocytes is due to additional stimuli such as direct cell-cell contact or other secreted factors.

Such a role of the MSCs as trophic mediators in cartilage formation in coculture pellets is in line with their proposed role in tissue repair in other tissues, such as brain [47-48], heart [49-51] [52], and kidney regeneration [53-54]. By providing nutrients and growth factors, MSCs increase proliferation and differentiation of hostderived cells to help them to repair damaged tissues [55]. The results of the present study are in line with and extent these observations to cartilage tissue formation. We are the first to show that MSCs have a prominent role as trophic mediators to stimulate cartilage matrix formation in pellet co-cultures with chondrocytes.

Despite the success of ACI in treatment of large-size cartilage defects, the requirement of two operations separated by several weeks' expansion of chondrocytes in vitro, is a major drawback of this procedure [56]. The results of this study imply that culture expansion of chondrocytes may benefit from co-culturing with MSCs. The MSCs may not only stimulate proliferation, thereby shortening culture time, but simultaneously may help the chondrocytes to retain their phenotype by counteracting chondrocyte dedifferentiation [41-42]. They further imply that a substantial part of the chondrocytes needed for ACI may be substituted with MSCs without decrease in cartilage matrix formation. This may pave the road for a single step surgery to repair large-size cartilage defects, in which chondrocytes are isolated, mixed with bone marrow cells from the same patient, loaded on a scaffold and directly re-implanted into the patient. Based on our ex vivo results, one may expect that in a few weeks the implant will consist mainly of chondrocytes and cartilage specific matrix.

In conclusion, our data clearly demonstrate that in pellet co-cultures of MSCs and primary chondrocytes, MSCs disappear over time. Increased cartilage formation in these co-cultures is mainly due to a trophic role of the MSCs in stimulating chondrocyte proliferation and matrix deposition by chondrocytes rather than MSCs actively undergoing chondrogenic differentiation. 


\section{Reference}

1. Brittberg M, Lindahl A, Nilsson A, et al. Treatment of deep cartilage defects in the knee with autologous chondrocyte transplantation. N Engl J Med 1994;331:889-895.

2. Peterson L, Minas T, Brittberg M, et al. Treatment of osteochondritis dissecans of the knee with autologous chondrocyte transplantation: results at two to ten years. J Bone Joint Surg Am 2003;85-A Suppl 2:17-24.

3. Schnabel M, Marlovits S, Eckhoff G, et al. Dedifferentiation-associated changes in morphology and gene expression in primary human articular chondrocytes in cell culture. Osteoarthritis Cartilage 2002;10:62-70.

4. Gruber HE, Deepe R, Hoelscher GL, et al. Human adipose-derived mesenchymal stem cells: direction to a phenotype sharing similarities with the disc, gene expression profiling, and coculture with human annulus cells. Tissue Eng Part A 2010;16:2843-2860.

5. Mo XT, Guo SC, Xie HQ, et al. Variations in the ratios of co-cultured mesenchymal stem cells and chondrocytes regulate the expression of cartilaginous and osseous phenotype in alginate constructs. Bone 2009;45:42-51.

6. Tsuchiya K, Chen G, Ushida T, et al. The effect of coculture of chondrocytes with mesenchymal stem cells on their cartilaginous phenotype in vitro. Materials Science \& Engineering CBiomimetic and Supramolecular Systems 2004;24:6.

7. Hendriks J, Riesle J, van Blitterswijk CA. Co-culture in cartilage tissue engineering. J Tissue Eng Regen Med 2007;1:170-178.

8. Bigdeli N, Karlsson C, Strehl R, et al. Coculture of human embryonic stem cells and human articular chondrocytes results in significantly altered phenotype and improved chondrogenic differentiation. Stem Cells 2009;27:1812-1821.

9. Gunja NJ, Athanasiou KA. Effects of co-cultures of meniscus cells and articular chondrocytes on PLLA scaffolds. Biotechnol Bioeng 2009;103:808-816.

10. Hildner F, Concaro S, Peterbauer A, et al. Human adipose-derived stem cells contribute to chondrogenesis in coculture with human articular chondrocytes. Tissue Eng Part A 2009;15:39613969.

11. Hendriks J, Miclea R, Schotel R, et al. Primary chondrocytes enhance cartilage tissue formation upon co-culture with a range of cell types. Soft Matter 2010;in press:DOI: 10.1039/C1030SM00266F.

12. Jiang Y, Jahagirdar BN, Reinhardt RL, et al. Pluripotency of mesenchymal stem cells derived from adult marrow. Nature 2002;418:41-49.

13. Bruder SP, Fink DJ, Caplan AI. Mesenchymal stem cells in bone development, bone repair, and skeletal regeneration therapy. J Cell Biochem 1994;56:283-294.

14. da Silva Meirelles L, Caplan AI, Nardi NB. In search of the in vivo identity of mesenchymal stem cells. Stem Cells 2008;26:2287-2299.

15. Dai W, Hale SL, Martin BJ, et al. Allogeneic mesenchymal stem cell transplantation in postinfarcted rat myocardium: short- and long-term effects. Circulation 2005;112:214-223.

16. Noiseux N, Gnecchi M, Lopez-Ilasaca M, et al. Mesenchymal stem cells overexpressing Akt dramatically repair infarcted myocardium and improve cardiac function despite infrequent cellular fusion or differentiation. Mol Ther 2006;14:840-850.

17. Crigler L, Robey RC, Asawachaicharn A, et al. Human mesenchymal stem cell subpopulations express a variety of neuro-regulatory molecules and promote neuronal cell survival and neuritogenesis. Exp Neurol 2006;198:54-64.

18. Gnecchi M, He H, Liang OD, et al. Paracrine action accounts for marked protection of ischemic heart by Akt-modified mesenchymal stem cells. Nat Med 2005;11:367-368.

19. Hwang NS, Varghese S, Puleo C, et al. Morphogenetic signals from chondrocytes promote chondrogenic and osteogenic differentiation of mesenchymal stem cells. J Cell Physiol 2007;212:281-284.

20. Chen WH, Lai MT, Wu AT, et al. In vitro stage-specific chondrogenesis of mesenchymal stem cells committed to chondrocytes. Arthritis Rheum 2009;60:450-459.

21. Hendriks J, Riesle J, Vanblitterswijk CA. Effect of stratified culture compared to confluent culture in monolayer on proliferation and differentiation of human articular chondrocytes. Tissue Eng 2006;12:2397-2405.

22. Fernandes H, Dechering K, Van Someren E, et al. The role of collagen crosslinking in differentiation of human mesenchymal stem cells and MC3T3-E1 cells. Tissue Eng Part A 
2009; 15:3857-3867.

23. Abramoff M, Magelhaes P, Ram S. Image Processing with ImageJ. Biophotonics International 2004;11:8.

24. Katopodi T, Tew SR, Clegg PD, et al. The influence of donor and hypoxic conditions on the assembly of cartilage matrix by osteoarthritic human articular chondrocytes on Hyalograft matrices. Biomaterials 2009;30:535-540.

25. Steinberg MS. Differential adhesion in morphogenesis: a modern view. Curr Opin Genet Dev 2007; 17:281-286.

26. Waschbisch A, Meuth SG, Herrmann AM, et al. Intercellular exchanges of membrane fragments (trogocytosis) between human muscle cells and immune cells: a potential mechanism for the modulation of muscular immune responses. J Neuroimmunol 2009;209:131-138.

27. Tajbakhsh S, Vivarelli E, Cusella-De Angelis G, et al. A population of myogenic cells derived from the mouse neural tube. Neuron 1994;13:813-821.

28. Vadala G, Studer RK, Sowa G, et al. Coculture of bone marrow mesenchymal stem cells and nucleus pulposus cells modulate gene expression profile without cell fusion. Spine (Phila Pa 1976) 2008;33:870-876.

29. Kuan WL, Hurelbrink CB, Barker RA. Increased capacity for axonal outgrowth using xenogenic tissue in vitro and in a rodent model of Parkinson's disease. Xenotransplantation 2006;13:233247.

30. Wang L, Li L, Menendez P, et al. Human embryonic stem cells maintained in the absence of mouse embryonic fibroblasts or conditioned media are capable of hematopoietic development. Blood 2005;105:4598-4603.

31. Wolbank S, Stadler G, Peterbauer A, et al. Telomerase immortalized human amnion- and adiposederived mesenchymal stem cells: maintenance of differentiation and immunomodulatory characteristics. Tissue Eng Part A 2009;15:1843-1854.

32. Salic A, Mitchison TJ. A chemical method for fast and sensitive detection of DNA synthesis in vivo. Proc Natl Acad Sci U S A 2008;105:2415-2420.

33. Foty RA, Steinberg MS. The differential adhesion hypothesis: a direct evaluation. Dev Biol 2005;278:255-263.

34. Nelson CM, Jean RP, Tan JL, et al. Emergent patterns of growth controlled by multicellular form and mechanics. Proc Natl Acad Sci U S A 2005;102:11594-11599.

35. Secchiero P, Melloni E, Corallini F, et al. Tumor necrosis factor-related apoptosis-inducing ligand promotes migration of human bone marrow multipotent stromal cells. Stem Cells 2008;26:29552963.

36. Chen XD. Extracellular matrix provides an optimal niche for the maintenance and propagation of mesenchymal stem cells. Birth Defects Res C Embryo Today 2010;90:45-54.

37. Choi KH, Choi BH, Park SR, et al. The chondrogenic differentiation of mesenchymal stem cells on an extracellular matrix scaffold derived from porcine chondrocytes. Biomaterials 2010;31:53555365 .

38. Werb Z. ECM and cell surface proteolysis: regulating cellular ecology. Cell 1997;91:439-442.

39. Liu X, Hou J, Shi L, et al. Lysophosphatidic acid protects mesenchymal stem cells against ischemia-induced apoptosis in vivo. Stem Cells Dev 2009;18:947-954.

40. Song IH, Caplan AI, Dennis JE. Dexamethasone inhibition of confluence-induced apoptosis in human mesenchymal stem cells. J Orthop Res 2009;27:216-221.

41. Banas A, Teratani T, Yamamoto Y, et al. IFATS collection: in vivo therapeutic potential of human adipose tissue mesenchymal stem cells after transplantation into mice with liver injury. Stem Cells 2008;26:2705-2712.

42. Weil BR, Markel TA, Herrmann JL, et al. Mesenchymal stem cells enhance the viability and proliferation of human fetal intestinal epithelial cells following hypoxic injury via paracrine mechanisms. Surgery 2009;146:190-197.

43. Namba A, Aida Y, Suzuki N, et al. Effects of IL-6 and soluble IL-6 receptor on the expression of cartilage matrix proteins in human chondrocytes. Connect Tissue Res 2007;48:263-270.

44. Csaki C, Matis U, Mobasheri A, et al. Co-culture of canine mesenchymal stem cells with primary bone-derived osteoblasts promotes osteogenic differentiation. Histochem Cell Biol 2009;131:251266.

45. $\mathrm{Xu} \mathrm{J,} \mathrm{Liu} \mathrm{X,} \mathrm{Chen} \mathrm{J,} \mathrm{et} \mathrm{al.} \mathrm{Cell-cell} \mathrm{interaction} \mathrm{promotes} \mathrm{rat} \mathrm{marrow} \mathrm{stromal} \mathrm{cell} \mathrm{differentiation}$ into endothelial cell via activation of TACE/TNF-alpha signaling. Cell Transplant 2010;19:43-53. Zhang B, Liu R, Shi D, et al. Mesenchymal stem cells induce mature dendritic cells into a novel 


\section{Chapter 3}

Jagged-2-dependent regulatory dendritic cell population. Blood 2009;113:46-57.

47. Chen J, Li Y, Katakowski M, et al. Intravenous bone marrow stromal cell therapy reduces apoptosis and promotes endogenous cell proliferation after stroke in female rat. J Neurosci Res 2003;73:778-786.

48. Li Y, Chen J, Zhang CL, et al. Gliosis and brain remodeling after treatment of stroke in rats with marrow stromal cells. Glia 2005;49:407-417.

49. Kinnaird T, Stabile E, Burnett MS, et al. Local delivery of marrow-derived stromal cells augments collateral perfusion through paracrine mechanisms. Circulation 2004;109:1543-1549.

50. Tang YL, Zhao Q, Zhang YC, et al. Autologous mesenchymal stem cell transplantation induce VEGF and neovascularization in ischemic myocardium. Regul Pept 2004;117:3-10.

51. Zhang M, Mal N, Kiedrowski M, et al. SDF-1 expression by mesenchymal stem cells results in trophic support of cardiac myocytes after myocardial infarction. FASEB J 2007;21:3197-3207.

52. Kawada H, Fujita J, Kinjo K, et al. Nonhematopoietic mesenchymal stem cells can be mobilized and differentiate into cardiomyocytes after myocardial infarction. Blood 2004;104:3581-3587.

53. Baer PC, Geiger H. Mesenchymal stem cell interactions with growth factors on kidney repair. Curr Opin Nephrol Hypertens 2010;19:1-6.

54. Imai N, Kaur T, Rosenberg ME, et al. Cellular therapy of kidney diseases. Semin Dial 2009;22:629-635.

55. Caplan AI, Dennis JE. Mesenchymal stem cells as trophic mediators. J Cell Biochem 2006;98:1076-1084.

56. Ruano-Ravina A, Jato Diaz M. Autologous chondrocyte implantation: a systematic review. Osteoarthritis Cartilage 2006;14:47-51. 


\section{Supplementary materials}

\section{Methods}

Quantitative PCR (qPCR) was performed on genomic DNA or cDNA samples by using the iQ SYBR Green Supermix (Bio-Rad, Hercules, CA). PCR Reactions were carried out on MyiQ2 Two-Color Real-Time PCR Detection System (Bio-Rad, Hercules, $\mathrm{CA}$ ) with the following conditions: cDNA was denatured for $5 \mathrm{~min}$ at $95^{\circ} \mathrm{C}$, followed by 45 cycles, consisting $15 \mathrm{~s}$ at $95^{\circ} \mathrm{C}, 15 \mathrm{~s} 60^{\circ} \mathrm{C}$ and $30 \mathrm{~s}$ at $72^{\circ} \mathrm{C}$. For each reaction a melting curve was generated to test primer dimer formation and non-specific priming. For each gene standard curves were obtained by serial dilutions of DNA and qPCR using species specific primers and the cross species specific primers. Bio-Rad iQ5 optical system software (version 2.0) was used to calculate copy numbers for each condition using the standard curve as reference. The relative signal was, subsequently defined as the proportion of human or bovine GAPDH copy numbers as percentage of the total copy numbers of both human and bovine genes. Calculation of Relative Expression was performed with Bio-Rad iQ5 optical system software (version 2.0) using the double delta $\mathrm{Ct}$ method [1]. Cross species specific GAPDH and $\beta$-actin primers were both used for normalization. All qPCR results were substrated by background sigals.

Figure S 1. Characteristics of iMSCs. Telomerase immortalized human mesenchymal stem cells (iMSCs) were made by infecting primary MSCs with a retrovirus encoding hTERT. After selection by neomycin, a subclone was selected and cultured in $\alpha$-MEM supplemented with 10\% FBS, 0.2mM Ascorbic acid 2phosphate, $100 \mathrm{U}$ penicillin $/ \mathrm{ml}$ and $100 \mu \mathrm{g} / \mathrm{ml}$ streptomycin. The generation and initial characterization of the iMSCs are described elsewhere (Noordhuis et al. in preparation) and partly summarized. (A) Surface markers expression was examined by FACs. iMSCs were harvested and incubated with PBS (black graph) or a primary antibody conjugated with FITC (green graph) or PE (Phycoerythrin, red graph). Antibodies against CD 24, CD 29, CD44, CD 81, CD 166 and CD 200 were purchased from BD Biosciences (Franklin Lakes, NJ). (B) Morphology of iMSCs in culture medium. Scale bar=200 $\mu \mathrm{m}$. (C) Adipogenic differentiation. iMSCs were seeded at density of 12000 cells $/ \mathrm{cm}^{2}$ and cultured in adipogenic medium ( $\alpha$-MEM supplemented with $10 \%$ FBS, $10^{-6} \mathrm{M}$ of dexamethasone, $10 \mu \mathrm{M}$ of Insulin, $0.5 \mathrm{mM}$ of IBMX (isobutylmethylxanthine), $200 \mu \mathrm{M}$ of Indomethacin, $100 \mathrm{U}$ penicillin $/ \mathrm{ml}$ and $100 \mu \mathrm{g} / \mathrm{ml}$ streptomycin) for 2 weeks. Fat droplets were visualized by Oil Red O staining. Scale bar=100 $\mu$ m. (D) Osteogenic differentiation. iMSCs were seeded at density of 12 


\section{Chapter 3}

000 cells $/ \mathrm{cm}^{2}$ and cultured in osteogenic medium ( $\alpha$-MEM supplemented with $10 \% \mathrm{FBS}, 0.2 \mathrm{mM}$ AsAP, $10^{-7} \mathrm{M}$ of dexamethasone, $5 \mathrm{mM}$ of $\beta$-GP ( $\beta$-glycerophosphate), $100 \mathrm{U}$ penicillin $/ \mathrm{ml}$ and $100 \mu \mathrm{g} / \mathrm{ml}$ streptomycin) for 3 weeks. Mineralized nodules were visualized by Alizarine Red staining. Scale bar $=100 \mu \mathrm{m}$. (E) Chondrogenic differentiation. 200000 of iMSCs were seeded per well in 96-well plate. Cell pellets were made by centrifuge at $500 \mathrm{~g}$ for $3 \mathrm{~min}$. Then pellets were cultured in chondrogenic differentiation medium (DMEM supplemented with $40 \mathrm{ug} / \mathrm{mL}$ of proline, $50 \mathrm{ug} / \mathrm{mL}$ ITS-premix, $50 \mathrm{ug} / \mathrm{mL}$ of AsAP, $100 \mathrm{ug} / \mathrm{mL}$ of Sodium Pyruvate, 10 $\mathrm{ng} / \mathrm{mL}$ of TGF $\beta 3$, 10-7 M of dexamethasone, $500 \mathrm{ng} / \mathrm{mL}$ of BMP6, $100 \mathrm{U}$ penicillin $/ \mathrm{ml}$ and $100 \mu \mathrm{g} / \mathrm{ml}$ streptomycin) for 3 weeks. Pellets were applied to histological examination as described in the Materials and Methods. Scale bar $=100 \mu \mathrm{m}$.

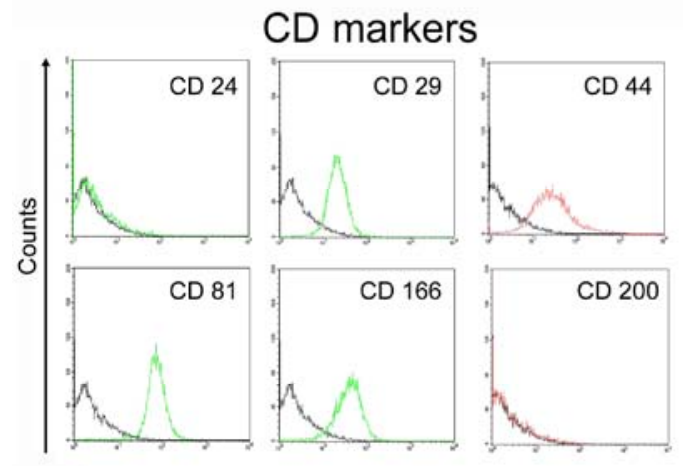

Morphology

Adipogenesis

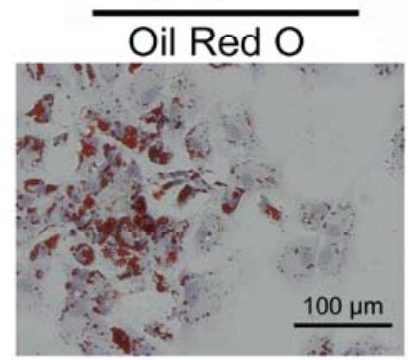

Osteogenesis

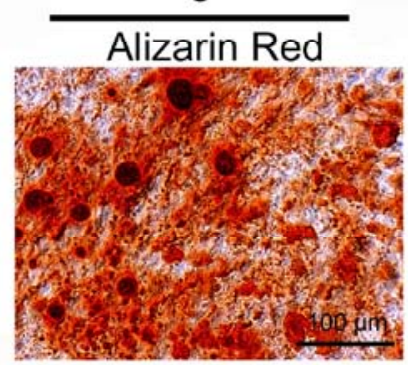

\section{Chondrogenesis}
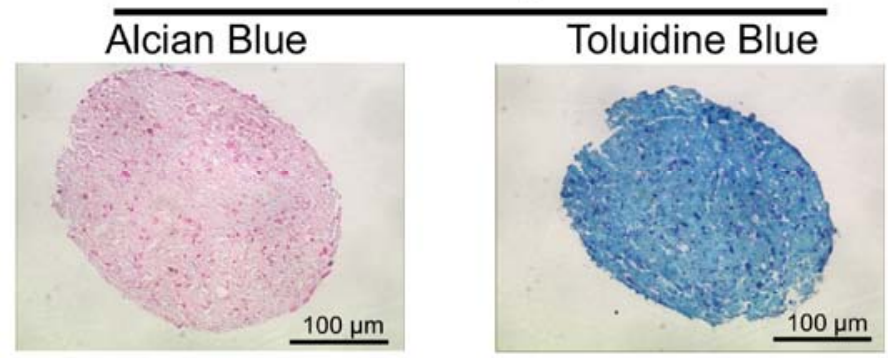

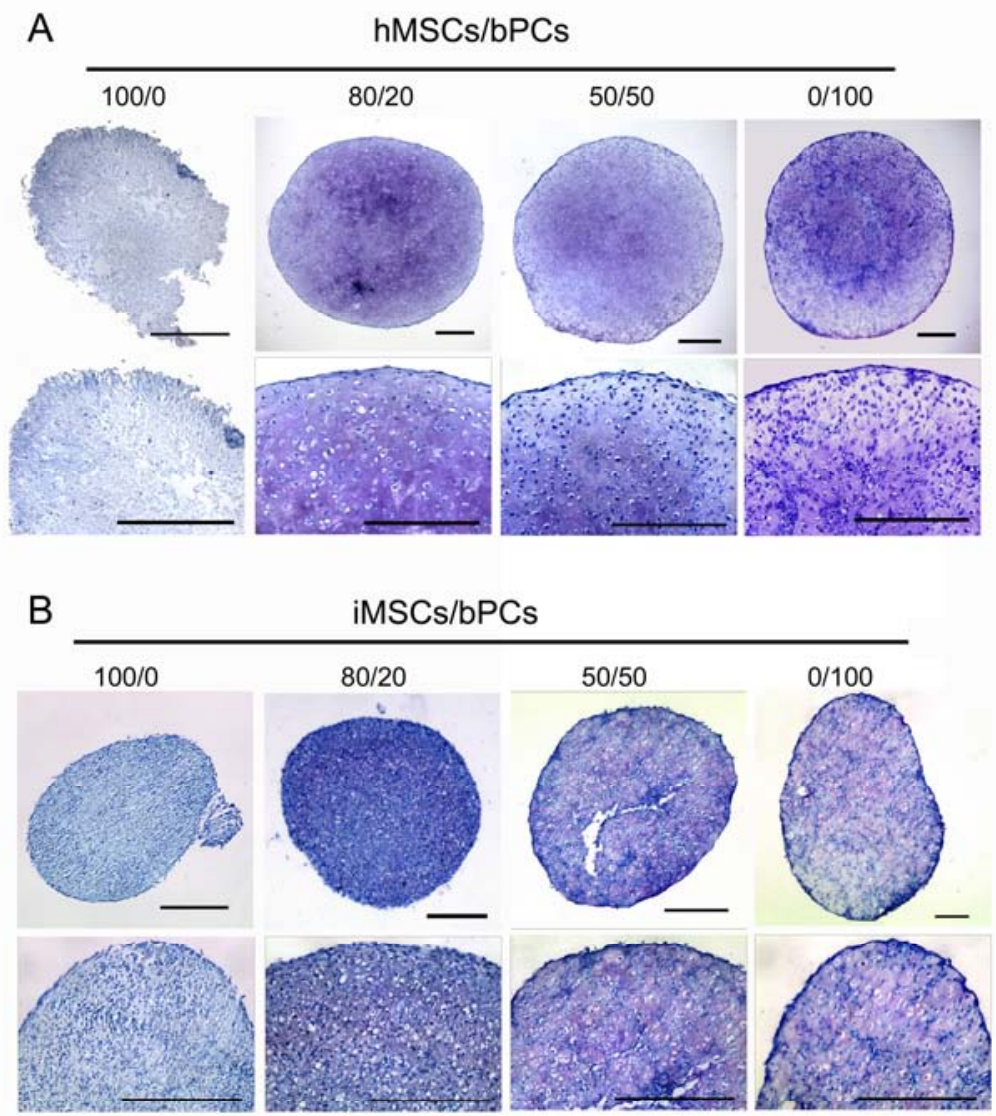

\section{C hMSCs in differentiation medium}

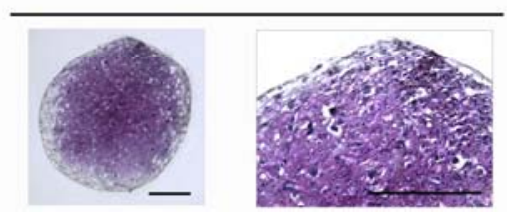

Figure $S$ 2. Toluidine blue staining shows the presence of GAG in pellets. (A-B) Toluidine blue staining confirms cartilage matrix formation. Ratios of hMSCs and bPCs (A) or iMSCs and bPCs (B) were indicated on the left of the figure. Upper panel shows an overview of pellets, while lower panel shows magnified pictures. Pellets of 200000 cells were cultured in chondrocyte proliferation medium for 4 weeks before histological staining. Scale bar $=200 \mu \mathrm{m}$. (C) hMSCs from the same donor as shown in figure $1 \mathrm{~A}$ and B had capacity of chondrogenic differentiation. Pellets of 200000 cells were cultured in chondrogenic differentiation medium (DMEM supplemented with $40 \mathrm{ug} / \mathrm{mL}$ of praline, $50 \mathrm{ug} / \mathrm{mL}$ ITS-premix, $50 \mathrm{ug} / \mathrm{mL}$ of AsAP, 100 $\mathrm{ug} / \mathrm{mL}$ of Sodium Pyruvate, $10 \mathrm{ng} / \mathrm{mL}$ of TGF- $\beta$ 3, 10-7 M of dexamethasone, $100 \mathrm{U}$ penicillin/ml and $100 \mu \mathrm{g} / \mathrm{ml}$ streptomycin) for 3 weeks before histological examination. Scale bar $=200 \mu \mathrm{m}$. 


\section{Chapter 3}

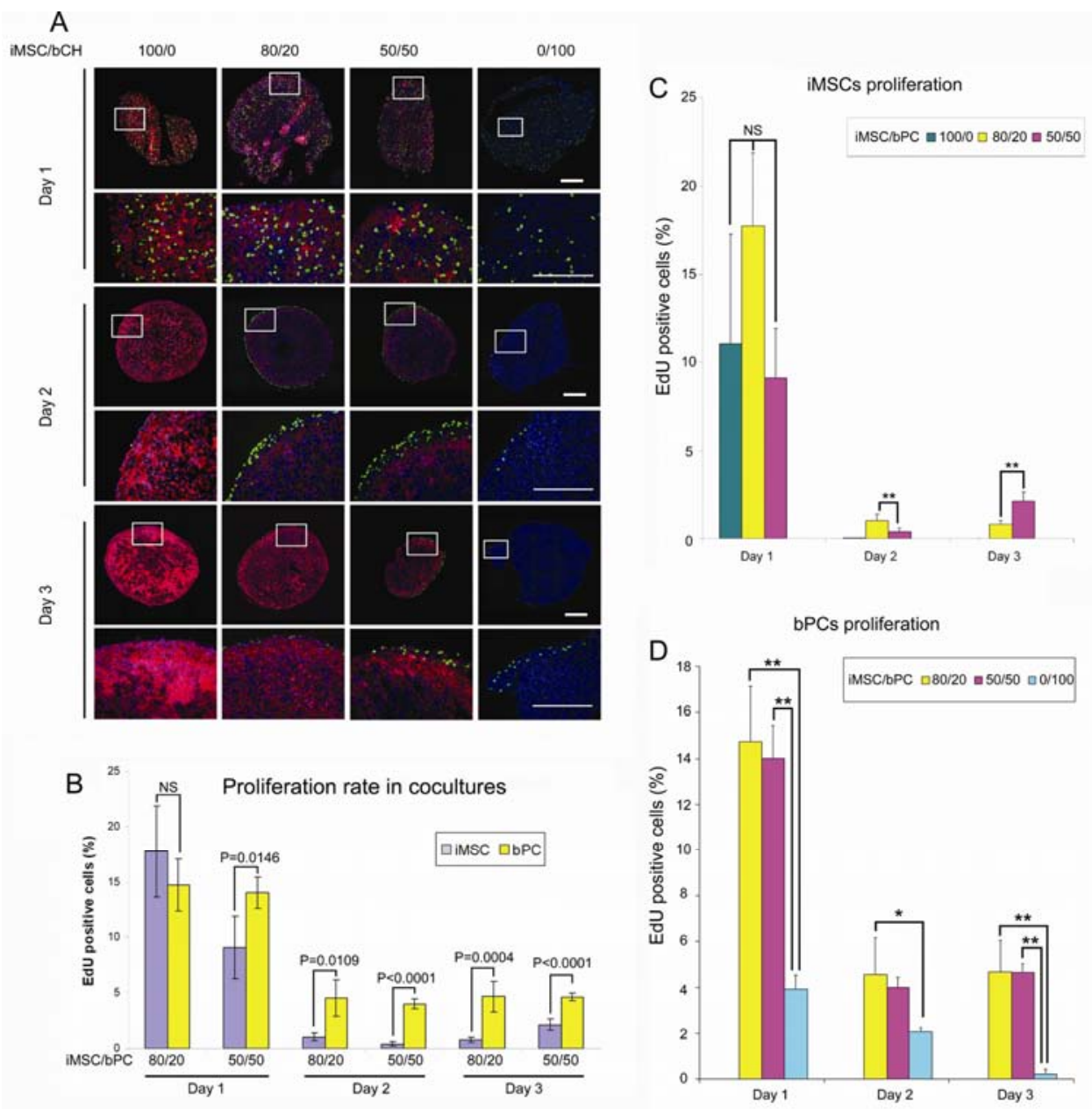

Figure S 3. Proliferation rate of bPCs is higher than that of iMSCs in co-cultures. (A) EdU staining of pellets at day 2 and day 3. iMSCs were labeled with CM-DiI (red), EdU was incorporated into newly

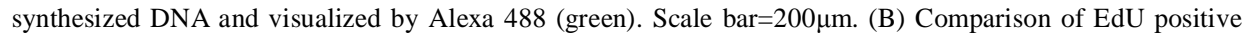
cells between iMSCs and bPCs in coculture pellets. Calculation of EdU positive cells was described in Materials and Methods. $P$ values were calculated by Student's test. NS=non-significance. Error bar reflects Standard Deviation (S. D.). (C) Quantification of EdU positive iMSCs in all conditions. The initial ratios of iMSCs and bPCs are indicated by bar colors. Asterisk represents $P<0.05$. Double asterisk represents $P<0.01$. NS=Non Significance. Error bar reflects Standard Deviation (S. D.). (D) Quantification of EdU positive bPCs in all conditions. The initial ratios of iMSCs and bPCs are indicated by bar colors. Asterisk represents $P<0.05$. Double asterisk represents $P<0.01$. NS=Non Significance. Error bar reflects Standard Deviation (S. D.). 
Table S1. Donor information of human primary cells

\begin{tabular}{llll}
\hline $\begin{array}{l}\text { Origin of biopsy } \\
\text { knee }\end{array}$ & Gender & Age (years) & $\begin{array}{l}\text { Remarks } \\
\text { Chondrocytes used in co-cultures of hMSCs } \\
\text { and hPCs (Fig. 1) }\end{array}$ \\
\hline Bone marrow & Female & 60 & $\begin{array}{l}\text { MSCs used in co-cultures of hMSCs and } \\
\text { hPCs (Fig. 1) }\end{array}$ \\
Bone marrow & Female & 66 & $\begin{array}{l}\text { MSCs use in co-cultures of hMSCs and } \\
\text { bPCs (Fig. 2) }\end{array}$ \\
\hline
\end{tabular}

Table S2. Forward (F) and Reverse (R) primers used for quantitative PCR on genomic DNA.

\begin{tabular}{|c|c|c|c|}
\hline Gene Name & Primer Sequence & $\begin{array}{l}\text { Product } \\
\text { size }\end{array}$ & Gene Bank No. \\
\hline \multirow{2}{*}{ Cross-species GAPDH } & F: 5' GCATTGCCCTCAACGACCA 3 ' & 179 or & NC_000012 \\
\hline & R: 5' CACCACCCTGTTGCTGTAGCC 3' & $171 \#$ & \& NC_007303 \\
\hline specific & F: 5' TTCCACCCATGGCAAATTCC 3' & 131 & NC_000012 \\
\hline GAPDH & R: 5' TTGCCTCCCCAAAGCACATT 3' & & \\
\hline specific & F: 5' AGCCGCATCCCTGAGACAAG 3' & 132 & NC_007303 \\
\hline GAPDH & R: 5' CAGAGACCCGCTAGCGCAAT 3' & & \\
\hline
\end{tabular}

\#Product size of human genomic GAPDH is 179, of bovine genomic GAPDH is 171 .

Table S3. Forward (F) and Reverse $(\mathrm{R})$ primers used for quantitative RT-PCR

\begin{tabular}{|c|c|c|c|c|}
\hline \multicolumn{2}{|l|}{ Gene Name } & Primer Sequence & $\begin{array}{l}\text { Product } \\
\text { size }\end{array}$ & Gene Bank No. \\
\hline \multirow{2}{*}{\multicolumn{2}{|c|}{ Cross-species $\beta$-Actin }} & F: 5' GCGCAAGTACTCCGTGTGGA 3' & 123 & NM_001101 \& \\
\hline & & R: 5' AAGCATTTGCGGTGGACGAT 3' & & NM_173979 \\
\hline \multirow{2}{*}{\multicolumn{2}{|c|}{ Cross-species GAPDH }} & F: 5’ AGCTCACTGGCATGGCCTTC 3' & 116 & NM_002046\& \\
\hline & & R: 5' CGCCTGCTTCACCACCTTCT 3' & & NM_001034034 \\
\hline Human & \multirow[t]{2}{*}{ specific } & F: 5’ CGCTCTCTGCTCCTCCTGTT 3’' & 82 & NM_002046 \\
\hline GAPDH & & R: 5'CCATGGTGTCTGAGCGATGT 3' & & \\
\hline Bovine & \multirow[t]{2}{*}{ specific } & F: 5' GCCAT CACTG CCACC CAGAA 3' & 207 & NM_001034034 \\
\hline GAPDH & & R: 5' GCGGCAGGTCAGATCCACAA 3' & & \\
\hline Human & \multirow[t]{2}{*}{ specific } & F: 5' TTCCCATCGTGCCTTTCCA 3' & 121 & NM_013227 \\
\hline Aggrecan & & R: 5'AACCAACGATTGCACTGCTCTT 3' & & \\
\hline Bovine & \multirow[t]{2}{*}{ specific } & F: 5' CCAAGCTCTGGGGAGGTGTC 3' & 98 & NM_173981 \\
\hline Aggrecan & & R: 5’ GAGGGCTGCCCACTGAAGTC 3' & & \\
\hline Human & \multirow[t]{2}{*}{ specific } & F: 5' GGCGGGGAGAAGACGCAGAG 3' & 129 & NM_001844 \\
\hline Collagen II & & R: 5' CGCAGCGAAACGGCAGGA 3' & & \\
\hline Bovine & \multirow{2}{*}{ specific } & F: 5' AGGTCTGACTGGCCCCATTG 3' & 101 & NM_001001135 \\
\hline Collagen II & & R: 5' CTCGAGCACCAGCAGTTCCA 3' & & \\
\hline \multirow{2}{*}{$\begin{array}{l}\text { Human } \\
\text { collagen IX }\end{array}$} & \multirow[t]{2}{*}{ specific } & F: 5’ GGCAGAAATGGCCGAGACG 3' & 150 & NM_001851 \\
\hline & & R: 5'CCCTTTGTTAAATGCTCGCTGA 3' & & \\
\hline \multirow{2}{*}{$\begin{array}{l}\text { Bovine } \\
\text { collagen IX }\end{array}$} & \multirow{2}{*}{ specific } & F: 5'GGACTCAACACGGGTCCACA 3' & 102 & XM_601325 \\
\hline & & R: 5' ACAGGTCCAGCAGGGCTTTG 3' & & \\
\hline
\end{tabular}


Table S4. Analysis of STR loci D5S818, D13S317, D16S539, CSF1PO and Penta_D on fully human samples

\begin{tabular}{|c|c|c|c|c|}
\hline Marker & $\begin{array}{l}\text { Original Ratio of } \\
\text { hMSCs and hPCs } \\
(\% / \%)\end{array}$ & $\begin{array}{l}\text { Area of hMSCs' } \\
\text { specific Allele }\end{array}$ & $\begin{array}{c}\text { Area of } \\
\text { hPCs' specific Allele }\end{array}$ & $\begin{array}{c}\text { Final Ratio of } \\
\text { hMSCs and } \\
\text { hPC }(\% / \%)\end{array}$ \\
\hline \multirow{4}{*}{ D5S818 } & $100 / 0$ & 3621,333 & 0 & $100 / 0$ \\
\hline & $80 / 20$ & 1963 & 1068,333 & $65 / 35$ \\
\hline & $50 / 50$ & 190 & 2670 & 7/93 \\
\hline & $0 / 100$ & 0 & 3695,25 & $0 / 100$ \\
\hline \multirow{4}{*}{ D13S317 } & $100 / 0$ & 3216 & 0 & $100 / 0$ \\
\hline & $80 / 20$ & 1270,333 & 1149,667 & $52 / 48$ \\
\hline & $50 / 50$ & 0 & 2525,333 & $0 / 100$ \\
\hline & $0 / 100$ & 0 & 4337,25 & $0 / 100$ \\
\hline \multirow{4}{*}{ D16S539 } & $100 / 0$ & 6722,333 & 0 & $100 / 0$ \\
\hline & $80 / 20$ & 2190 & 1931 & $53 / 47$ \\
\hline & $50 / 50$ & 0 & 4365,8 & $0 / 100$ \\
\hline & $0 / 100$ & 0 & 9186,25 & $0 / 100$ \\
\hline \multirow{4}{*}{ CSF1PO } & $100 / 0$ & 5926,667 & 0 & $100 / 0$ \\
\hline & $80 / 20$ & 1438 & 1236,667 & $54 / 46$ \\
\hline & $50 / 50$ & 1103,8 & 6211,6 & $15 / 85$ \\
\hline & $0 / 100$ & 0 & 9398,5 & $0 / 100$ \\
\hline \multirow{4}{*}{ Penta_D } & $100 / 0$ & 14800,33 & 0 & $100 / 0$ \\
\hline & $80 / 20$ & 5979,333 & 2540,333 & $70 / 30$ \\
\hline & $50 / 50$ & 0 & 12163,8 & $0 / 100$ \\
\hline & $0 / 100$ & 0 & 13712,67 & $0 / 100$ \\
\hline
\end{tabular}

\section{Supplementary References:}

1. Livak KJ, Schmittgen TD. Analysis of relative gene expression data using realtime quantitative PCR and the 2(-Delta Delta C(T)) Method. Methods 2001;25:402-408. 


\title{
Chapter 4
}

\section{Trophic effects of mesenchymal stem cells in chondrocyte co-cultures are independent of culture conditions and cell sources*}

\author{
Ling Wu ${ }^{1}$, Henk-Jan Prins ${ }^{2,3}$, Marco N. Helder ${ }^{4}$, Clemens A. van Blitterswijk ${ }^{1}$, \\ Marcel Karperien ${ }^{1,5}$
}

1. Department of Tissue Regeneration, MIRA-Institute for Biomedical Technology and Technical Medicine, University of Twente, Enschede, 7522NB, the Netherlands.

2. Department of Oral Cell Biology, Academic Centre for Dentistry Amsterdam, Amsterdam, the Netherlands.

3. Department of Oral \& Maxillofacial Surgery, VU Medical Center, Amsterdam, The Netherlands

4. Department of Orthopaedics, VU Medical Center, Amsterdam, The Netherlands

5. Present address: Department of Developmental BioEngineering, MIRAInstitute for Biomedical Technology and Technical Medicine, University of Twente, Enschede, 7522 NB, the Netherlands. 


\section{ABSTRACT}

Previously we have shown that the increased cartilage production in pellet cocultures of chondrocytes and bone marrow derived mesenchymal stem cells (BM-MSCs) is due to a trophic role of the MSC in stimulating chondrocyte proliferation and matrix production rather than MSCs actively undergoing chondrogenic differentiation. These studies were performed in culture medium that was not compatible with chondrogenic differentiation of MSCs. In this study, we tested whether the trophic role of the MSCs is dependent on culturing co-culture pellets in medium compatible with chondrogenic differentiation of MSCs. In addition, we investigated whether the trophic role of the MSCs is dependent on their origins or is a more general characteristic of MSCs. Human BM-MSCs and bovine primary chondrocytes (bPCs) were co-cultured in medium compatible with chondrogenic differentiation of MSCs. Enhanced matrix production was confirmed by glycosaminoglycans (GAG) quantification. Species specific quantitative PCR (qPCR) demonstrated that cartilage matrix was mainly from bovine origin, indicative of a lack of chondrogenic differentiation of MSCs. In addition, pellet co-cultures were overgrown by bovine cells over time. To test the influence of origin on MSCs' trophic effects, MSCs isolated from adipose tissue and synovial membrane were co-cultured with human primary chondrocytes (hPCs) and their activity was compared with BM-MSCs, which served as control. GAG quantification again confirmed increased cartilage matrix production, irrespective of the source of the MSCs. EdU staining combined with cell tracking revealed increased proliferation of chondrocytes under each condition. Irrespective of the MSC source, short tandem repeat (STR) analysis of genomic DNA showed a decrease in MSCs in co-culture over time. Our results clearly demonstrate that in co-culture pellets MSCs stimulate cartilage formation due to a trophic effect on chondrocytes rather than differentiating into chondrocytes, irrespective of culture condition or origin. This implies that the trophic effect of MSCs in co-cultures is a general phenomenon with potential implications for use in cartilage repair strategies. 


\section{Introduction}

Despite the success of autologous chondrocyte implantation in treating largesize cartilage defects, there are some disadvantages of this treatment that limit its broader clinical application. One major issue is the requirement of relatively large quantities of chondrocytes from the patient[1], which are obtained by in vitro expansion. Partial replacement of the chondrocytes with mesenchymal stem cells (MSC) has been proposed to tackle this problem. Many studies have evaluated the feasibility of this idea by co-culturing of MSCs and chondrocytes[2]. Indeed, cartilage matrix deposition was found to be improved in co-cultures of MSCs and chondrocytes compared to cultures of pure chondrocytes or MSCs[3-4]. In our previous report[5], we have shown that pellet co-culture of bovine primary chondrocytes (bPCs) and human MSCs benefits cartilage matrix formation and that cartilage matrix genes were mainly expressed by bovine chondrocytes. In addition, we also showed that the ratio of MSCs decreased dramatically due to massive cell death of MSCs by apoptosis. Chondrocyte proliferation was increased either by co-culturing with MSCs or culturing with MSCs conditioned medium. These findings were also confirmed by an independent study performed by Acharya et al.(2011)[6]. This and our study demonstrated a new mechanism of cellular interaction in co-culture of MSCs and chondrocytes, in which the trophic effects of MSCs stimulate chondrocyte proliferation and cartilage matrix deposition rather than actively undergoing chondrogenic differentiation.

The concept of MSCs as a trophic mediator of tissue repair is introduced by Arnold Caplan [7], who borrowed the term trophic from neurobiology, in which it refers to bioactive molecules produced by nerve terminals, which are not neurotransmitters ${ }^{[8]}$. In relation to MSCs, the term trophic was first used to describe the process in which MSCs secrete factors that stimulate nearby cells to release functionally bioactive molecules ${ }^{[7]}$. Later, the term also relates to the effect of the factors produced by MSC on viability, proliferation, and matrix production of the neighboring cells. This concept has resulted in a paradigm shift in the way MSCs are involved in tissue repair. While traditionally it was believed that MSCs mainly repair damaged tissue by differentiating into specific cell types and replacing lost cells[9], nowadays the trophic role of the MSC in tissue repair is considered more important[10]. It was reported that MSCs, when 


\section{Chapter 4}

introduced either directly or systemically into stroked brain of rats, promote gain of coordinated functions without differentiating into neurons or any other supporting cell type[11]. Other examples are from the trophic role of MSCs in stimulating cardiomyocyte proliferation in vitro[12], and vascular regeneration in vivo[13].

So far, most of the reports regarding the trophic effects of MSCs are based on studies using bone marrow derived MSCs (BM-MSCs). There are many more sources from which MSCs can be isolated. For examples, adipose tissue can give rise to multipotent stromal cells [14-15], and human synovial membrane also contains a MSC population[16]. So far, MSCs have been isolated from many other tissues[17-18]. MSCs isolated from other sources share many common features with BM-MSCs[19-20], and have been applied in tissue engineering strategies[21-25]. Nevertheless, only a few papers documented the paracrine or autocrine effects of adipose tissue derived MSCs in tissue regeneration [26-27].The trophic effects of MSCs isolated from a non-bone marrow origin have not yet been studied systematically and thoroughly.

In a previous study, we have co-cultured human BM-MSCs and bPCs in pellets in chondrocyte proliferation medium[5], and showed that increased cartilage matrix formation was not due to chondrogenic differentiation of MSCs. This data indicated that in pellet co-cultures signals from the chondrocytes alone are insufficient to induce chondrogenic differentiation of MSCs at least under these culture conditions. In this study, we performed experiments in which BM-MSCs and bPCs were co-cultured in chondrogenic differentiation medium to investigate the influence of growth factors in culture medium on MSCs' trophic effects. In addition, we examined whether MSCs from multiple sources (bone marrow, adipose tissue and synovial membrane) have similar trophic effects by co-culturing them with human primary chondrocytes (hPCs). Our data presented here demonstrated that trophic effects of MSCs on chondrocytes in pellet co-cultures is a general feature of MSCs independent of culture conditions and their source.

\section{Materials and methods}

\section{Cell culture and expansion}

Bovine primary chondrocytes (bPCs) were isolated from full-thickness cartilage of knee biopsies from cows of approximately 6 months old. Human primary 
chondrocytes (hPCs) were obtained from macroscopically healthy cartilage obtained from knee or hip biopsies of patients with end stage osteoarthritis undergoing total knee or hip replacement. Both bovine and human cartilages were digested for $20-22 \mathrm{~h}$ in collagenase type II $(0.15 \%$ Worthington, NJ, US $)$ dissolved in chondrocytes proliferation medium. The components of chondrocytes proliferation medium are DMEM supplemented with 10\% FBS, $1 \times$ non-essential amino acids, $0.2 \mathrm{mM}$ Ascorbic acid 2-phosphate (AsAP), $0.4 \mathrm{mM}$ proline, 100U/ml penicillin and 100 $\mu \mathrm{g} / \mathrm{ml}$ streptomycin. More details about chondrocyte isolation are described elsewhere[28]. Bone marrow mesenchymal stem cells (BM-MSCs) were separated from human bone marrow aspirates as described previously[29]. Human adipose tissue mesenchymal stem cells (AT-MSCs) and synovium mesenchymal stem cells (Sy-MSCs) were isolated according to procedures in previous publications[30-31]. MSCs from all kinds of sources were cultured in MSC proliferation medium ( $\alpha$-MEM supplemented with $10 \%$ fetal bovine serum, $1 \%$ L-glutamin, $0.2 \mathrm{mM}$ ascorbic acid, $100 \mathrm{U} / \mathrm{ml}$ penicillin, 10 $\mu \mathrm{g} / \mathrm{ml}$ streptomycin and $1 \mathrm{ng} / \mathrm{ml} \mathrm{bFGF})$.

The use of all human materials in this study has been approved by a local Medical Ethical Committee. All reagents used for cell culture were purchased from Gibco, Invitrogen (Paisley, UK), unless otherwise stated. Common chemicals were purchased from Sigma-Aldrich.

\section{Pellet culture and chondrogenic differentiation}

For mono-cultures, 200,000 cells of hPCs or MSCs were seeded in one well of a round bottom 96 wells plate (non-tissue culture treated). For co-cultures, 200,000 cells were seeded in a $80 \%$ MSC / $20 \%$ hPC or bPC ratio. Cells were initially seeded in chondrocyte proliferation medium and centrifuged for $5 \mathrm{~min}$ at $500 \times \mathrm{g}$. Medium was changed to chondrogenic differentiation medium (DMEM supplemented with $40 \mu \mathrm{g} / \mathrm{mL}$ of proline, $50 \mathrm{ug} / \mathrm{mL}$ ITS-premix, $50 \mathrm{ug} / \mathrm{mL}$ of AsAP, $100 \mathrm{ug} / \mathrm{mL}$ of Sodium Pyruvate, $10 \mathrm{ng} / \mathrm{mL}$ of TGF $33,10^{-7} \mathrm{M}$ of dexamethasone, $500 \mathrm{ng} / \mathrm{mL}$ of BMP6, $100 \mathrm{U}$ penicillin/ml and $100 \mu \mathrm{g} / \mathrm{ml}$ streptomycin) one day after seeding when stable pellets were formed. Cell pellets were cultured for 4 weeks before analysis.

\section{Histology}

Cell pellets were fixed with $10 \%$ formalin for $15 \mathrm{~min}$, dehydrated with ethanol 


\section{Chapter 4}

and embedded in paraffin using routine procedures. A microtome (Shandon, France) was used to cut $5 \mu \mathrm{m}$ thick sections. Slides were then deparaffinized and stained for sulfated glycosaminoglycans (GAG) with Alcian blue. Nuclei were counterstained with nuclear fast red.

\section{Quantitative GAG and DNA assay}

Cell pellets $(n=6)$ were washed with phosphate-buffered saline (PBS) and stored at $-80^{\circ} \mathrm{C}$ for $16-20$ hours. Subsequently, they were digested in digestion buffer (1 $\mathrm{mg} / \mathrm{ml}$ proteinase $\mathrm{K}$ in Tris/EDTA buffer ( $\mathrm{pH}$ 7.6) containing $18.5 \mu \mathrm{g} / \mathrm{ml}$ iodoacetamide and $1 \mu \mathrm{g} / \mathrm{ml}$ pepstatin A) for more than $16 \mathrm{~h}$ at $56{ }^{\circ} \mathrm{C}$. GAG content was spectrophotometrically determined with 1,9-dimethylmethylene blue chloride (DMMB) staining in PBE buffer (14.2g/L Na $\mathrm{HPO}_{4}$ and 3.72g/L Na 2 EDTA, pH 6.5) using an ELISA reader (TECAN, Grodig, Austria) at an absorbance of $520 \mathrm{~nm}$ with chondroitin sulfate as a standard. Cell numbers were determined by quantification of total DNA using a CyQuant DNA Kit (Molecular Probes, Eugene, OR).

\section{DNA isolation, RNA isolation and quantitative PCR}

DNA samples of pellets were extracted with the DNA Mini Kit (Promega, Hilden, Germany) according to the manufacturer's protocols. RNA samples of cell pellets were isolated with the NucleoSpin RNA II Kit (Macherey-Nagel, Düren, Germany). Total RNA was reverse-transcribed into cDNA using the iScript cDNA Synthesis kit (Bio-Rad, Hercules, CA). Quantitative PCR (qPCR) was performed on genomic DNA or cDNA samples by using the iQ SYBR Green Supermix (Bio-Rad, Hercules, CA). PCR Reactions were carried out on MyiQ2 Two-Color Real-Time PCR Detection System (Bio-Rad, Hercules, CA) under the following conditions: cDNA was denatured for $5 \mathrm{~min}$ at $95^{\circ} \mathrm{C}$, followed by 45 cycles, consisting $15 \mathrm{~s}$ at $95^{\circ} \mathrm{C}, 15 \mathrm{~s} 60^{\circ} \mathrm{C}$ and $30 \mathrm{~s}$ at $72^{\circ} \mathrm{C}$. For each reaction a melting curve was generated to test primer dimer formation and non-specific priming. The sequences of primers for real-time PCR, either species specific or cross species specific were as previously described [5]. For PCR on genomic DNA, standard curves were generated by using serial dilutions of genomic DNA as template. Bio-Rad iQ5 optical system software (version 2.0) was used to calculate copy numbers for each condition using the standard curves as reference. The relative signal was defined as the proportion of human or bovine GAPDH copy numbers 
as percentage of the total copy numbers of both human and bovine genes. Calculation of Relative Expression was performed with Bio-Rad iQ5 optical system software (version 2.0) using the double delta $\mathrm{Ct}$ method[32]. Cross species specific GAPDH primers were used for normalization.

\section{Cell tracking with organic fluorescent dyes}

The organic fluorescent dye CM-DiI (Molecular Probes, Eugene, OR) was used for cell tracking in co-cultures. Cells were labeled according to the manufacturer's protocol. Briefly, cells were trypsinized and resuspended in PBS at a concentration of $2 \times 10^{6}$ cells $/ \mathrm{ml}$. The cells were incubated with $4 \mu \mathrm{M}$ of $\mathrm{CM}$-DiI at $37^{\circ} \mathrm{C}$ for 5 minutes followed by an incubation at $4^{\circ} \mathrm{C}$ for 15 minutes. Cells were washed with PBS and applied in co-culture experiments.

\section{5-ethynyl-2'-deoxyuridine labeling and staining}

Cell proliferation in pellets was examined with the Click-iT® EdU Imaging Kit (Molecular Probes, Eugene, OR). Cell pellets were cultured in chondrogenic differentiation medium containing $10 \mu \mathrm{M}$ EdU (5-ethynyl-2'-deoxyuridine) for 24 hours before harvesting. At day 2, cell pellets were washed with PBS and fixed with 10\% formalin for $15 \mathrm{~min}$. Sections of $10 \mu \mathrm{m}$ were cut with a cryotome (Shandon, France). Sections were permeabilized with $0.5 \%$ of Triton X 100 for 20 min and stained for EdU with Alexa 488 cocktail. Nuclei were counterstained with Hoechst 33342 (provided in the same kit).

\section{Image acquisition and analysis}

Histological images were made with a Nikon E300 microscope (Japan). Fluorescent images were taken with a BD pathway 435 confocal microscope (BD Biosciences, Rockville, MD). Details of images quantification were published elsewhere[5]. Values represent the mean \pm standard deviation of at least 3 biological replicates.

\section{Short Tandem Repeats (STR) analysis}

Genomic DNA samples were extracted from pellets with the QIAamp DNA Mini Kit (Qiagen). The sixteen loci of the kit PowerPlex 16 System (Promega) were amplified, typed, sequenced and analyzed by ServiceXS B.V. (Leiden, the Netherlands). Specific alleles for the donor of hMSCs and the donor of hPCs were found in several 
loci. These alleles were used to define the origin of cells in allogeneic co-culture of hMSCs and hPCs. The amount of DNA present for each donor was calculated from the areas of the electropherogram for each locus of hMSCs' or hPCs' specific alleles and the ratio of hMSCs and hPCs was determined.

\section{Statistical analysis}

GAG and DNA quantifications were examined for statistical significance with one-way analysis of variance (ANOVA) followed by Tukey HSD (Honestly Significant

Difference) Test. Statistical analysis of EdU positive cells was made by using the Student's $t$ test. $P$ values of $<0.05$ were considered as statistically significant.

\section{Results}

\section{Co-culture pellets in chondrogenic differentiation medium show increased cartilage formation}

To test if growth conditions, favorable for chondrogenic differentiation of MSCs, affects the trophic effects of MSCs in pellet co-cultures, we examined cartilage matrix formation of BM-MSCs and bPCs in chondrogenic differentiation medium. Each of the 3 BM-MSC donors were fully capable of differentiating into the chondrogenic, osteogenic and adipogenic lineage and expressed typical cell surface markers of MSCs (data not shown), although some variability was observed in the chondrogenic differentiation potential. After 4 weeks of co-culture, histology and GAG assays were performed to evaluate cartilage formation. As shown in figure 1A, the presence of GAG is indicated by Alcian blue staining in both mono-cultures and co-culture pellets. Cells in the positively stained areas were embedded in lacunae and show typical characteristics of chondrocytes, round shape and the presence of an Alcian blue positive extracellular matrix.. Similar data were obtained using Toluidine blue staining (data not shown). Quantitative analysis showed that both total GAG and total DNA increased as the ratio of MSCs decreased (figure. 1B). In line with previous studies, co-culture pellets contained more GAG than pellets seeded with $100 \%$ chondrocytes, when normalized to the initial seeding percentage of bPCs indicating that the beneficial effect on cartilage matrix formation in co-cultures is preserved when the pellets are cultured in growth factors containing medium. 


\section{Proliferation of chondrocytes causes ratio changes of two cell types in pellets cultured in chondrogenic differentiation medium}

Species specific qPCR was performed to study the origin of the chondrogenic marker genes in co-culture pellets (figure. 1C). At week 4, the expression levels of both human Aggrecan (ACAN) and human Collagen 2 (COL2) were much lower in coculture pellets than in pure MSCs pellets. On the other hand, the expression levels of bovine ACAN or COL2 in co-culture pellets were either higher or similar to that of pure bPCs pellets. These data indicated that the cartilaginous matrix in co-culture pellets is mainly from bovine origin. At week 4 of culture, genomic DNA was isolated from the cell pellets, and used as template in species specific qPCR. The ratio of human/bovine cells dropped from $80 \%$ (initial seeding percentage after 1 day) to approximately $40 \%$ after 4 weeks of culture (figure 1D).

Cell proliferation in co-culture pellets was studied using EdU incorporation combined with cell tracking. For this, bovine chondrocytes were labeled with CM-Dil (red). Proliferation was studied at day 2. As shown in figure 1E, EdU positive cells were mainly found in the periphery of the pellets. Cell tracking showed that co-culture predominantly increased proliferation of the chondrocytes with a minor effect on proliferation of the MSCs (figure 1F).

\section{Co-culture of hPCs and MSCs from multiple sources increases cartilage matrix formation}

To investigate whether the source of MSCs influences the degree of cartilage formation in co-culture pellets, MSCs derived from human adipose tissue and synovium were used in co-culture pellets with hPCs, while BM-MSCs served as control. Alcian blue staining shows deposition of GAGs to some extend in all groups (figure. 2A). GAG and DNA contents of each pellet were then determined by chemospectrophotometric and fluorescent assays (figure. 3). MSCs from different sources performed similarly in GAG formation when co-cultured with hPCs. An average of 3 donor pairs of MSCs and hPCs showed that co-culture pellets contained more GAG than $100 \%$ chondrocyte pellets, when normalized to the initial seeding percentage of hPCs. 

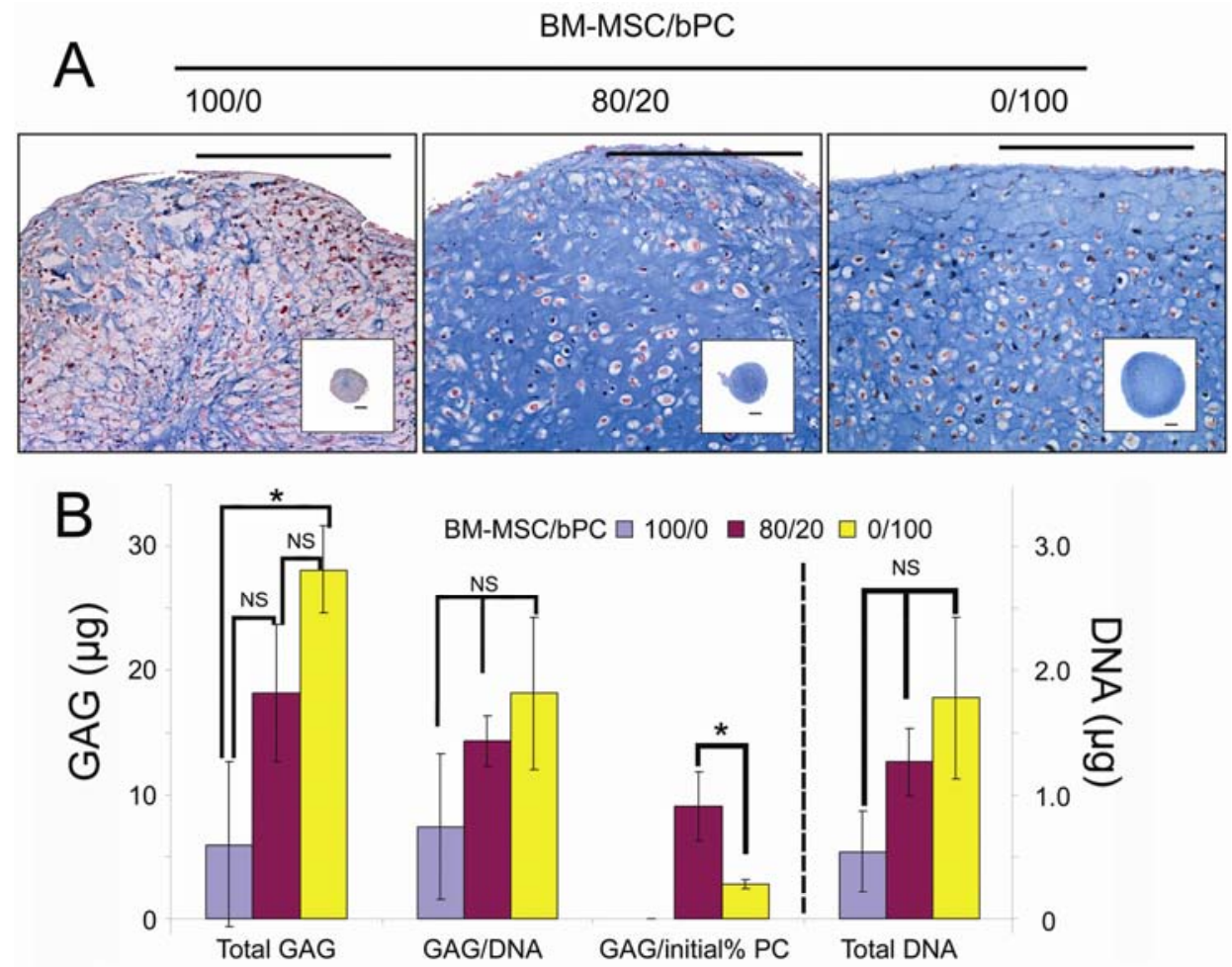

3.0
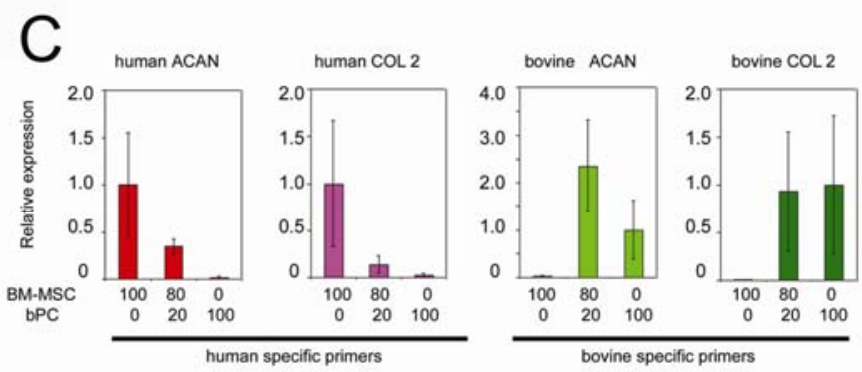

D $\square$ hGAPDH $\square$ bGAPDH
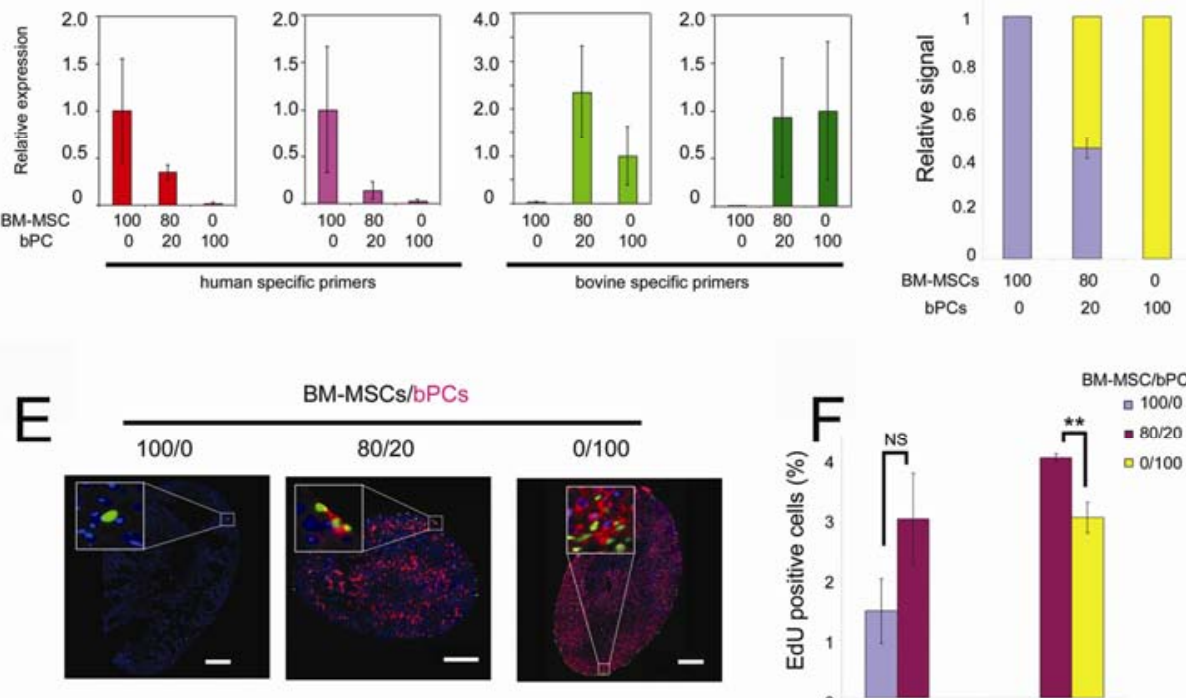

BM-MSCs/bPCs
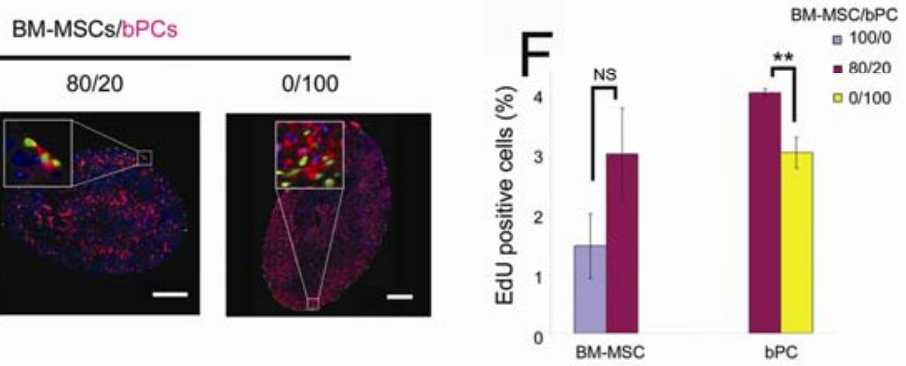
Figure 1: Cartilage matrix formation is increased in co-culture pellets cultured in chondrogenic differentiation medium; and enhanced cartilage matrix formation is caused by trophic effects of MSCs.. Pellets (200, 000cells) of BM-MSCs from 3 donors and bPC in 3 different ratios (100:0; 80:20 and 0:100 of BM-MSC:bPC) were cultured in medium compatible with chondrogenic differentiation of MSCs. After 4 weeks pellets were collected for histological analysis. (A) Alcian blue staining was performed to examine deposition of sulfated GAGs in midsagittal paraffin sections. Inserts indicate the overview of each pellet. Pictures show the results of a representative donor of MSCs, while 3 donors were tested. Scale bar=100 $\mu \mathrm{m}$. (B) GAG and DNA of cell pellets ( $n=6$ per donor) were quantitatively measured 4 weeks after co-culture. Averages of 3 donors of BM-MSCs are shown. Ratios of hMSCs and bPCs are indicated by the bar colors. The left y-axis is for "Total GAG", "GAG/DNA" and "GAG/initial \%PC", while the right y-scale is for "Total DNA”. Asterisk represents $P<0.05$. NS=Not Significant. Data are presented as a mean \pm standard deviation (S. D.). (C)Co-culture pellets of BM-MSCs and bPC were established and cultured in chondrogenic medium as described. After 4 weeks samples were collected for analysis of the expression levels of ACAN and COL2 mRNA by species specific qPCR. RNA samples were extracted from pellets ( $n=3$ per MSC donor). Relative expression levels were obtained by normalization of human or bovine specific signals to cross species-specific GAPDH. For human specific genes, values are relative amounts to 100/0 hMSC/bPC group. For bovine specific genes, values are relative amounts to $0 / 100 \mathrm{hMSC} / \mathrm{bPC}$ group. Data are presented as an average of 3 BM-MSCs donors \pm S. D. (D) After 4 weeks of culture species specific qPCR on genomic GAPDH was performed after DNA analysis ( $n=6$ per donor). Data represent the average of 3 BM-MSC donors \pm S.D. (E) After 2 days of culture, proliferation was assessed using an EdU assay. In this experiment, bPCs were labeled with CM-DiI (red). EdU incorporation into newly synthesized DNA was visualized by Alexa 488 (green) and nuclei were counterstained with Hoechst 33342 (blue) on $10 \mu \mathrm{M}$ freeze sections. Pictures show the results of a representative BM-MSC donor out of 3 donors tested. Scale bar=100 $\mu \mathrm{m}$. (F) Quantification of EdU positive cells. The initial ratios of hMSC and bPC are indicated by bar colors. Data from 3 donors of BM-MSCs measured in triplicate were analyzed for statistic significance. Double asterisk represents $P<0.01$. NS=Not Significant. Error bar reflects S. D. 

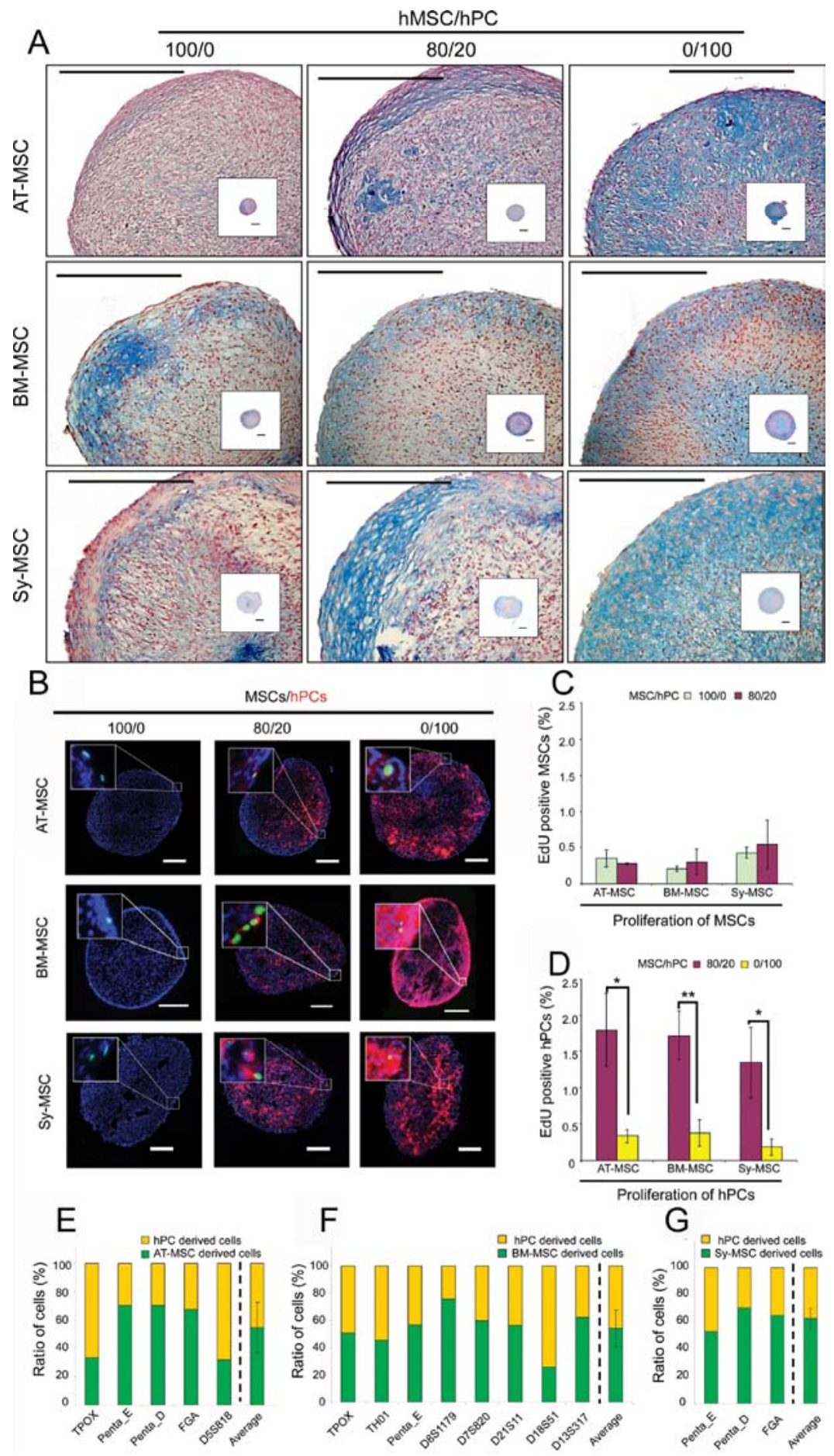

G

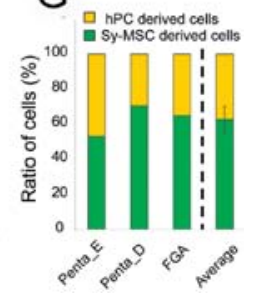


Figure 2 GAG formations in co-culture pellets of hPCs and MSCs from different sources; and MSCs from multiple sources act as trophic mediators when co-cultured with hPCs. (A) Human chondrocytes were co-cultured with MSCs from different sources in 3 different seeding ratios (MSC versus PC; 100:0; 80:20; 0:100) for 4 weeks in chondrogenic differentiation medium. Alcian blue staining was performed on midsagittal paraffin sections. Nuclei were counterstained with nuclear fast red. Pictures show a representative donor of MSCs, while 3 donors were tested. Scale bar=100 $\mu \mathrm{m}$. (B) Human chondrocytes were co-cultured in pellets with AT-MSCs, BM-MSCs or Sy-MSCs in 3 different ratios in chondrogenic differentiation medium as described in Materials and Methods. EdU staining was performed at day 2 to show proliferating cells in pellets. hPCs were labeled with CM-DiI (red). EdU incorporation into newly synthesized DNA was visualized by Alexa 488 (green). Nuclei were counterstained with Hoechst 33342 (blue). Sources of MSCs are indicated on the left. Pictures are representative for each condition (3 pellets analyzed per donor) and are $10 \mu \mathrm{m}$ mid-sagittal freeze sections. Insets are magnifications of the boxed areas. Scale bar $=100 \mu \mathrm{m}$. (C) and (D) Quantification of EdU positive MSCs (C) or hPCs (D). The initial ratios of MSC and bPC are indicated by bar colors. The sources of MSCs are indicated at the bottom. Data represent the average from 3 donors of MSCs, each measured in triplicate +/- S.D. Asterisk represents $P<0.05$. Double asterisk represents $P<0.01$. (E), (F and (G) STR analysis was performed on genomic DNA isolated from co-culture pellets of MSCs from adipose tissue (E), bone marrow (F) or synovium (G) and hPCs at an initial seeding ratio of 80:20 after 4 weeks of culture. Only informative loci that could discriminate between the MSCs and PCs are shown. The loci are listed on the bottom of figure. An average of these loci is shown by the rightmost bar +/- S.D. Data showed here is representative from 1 donor pair of MSCs and PCs, while three donor pairs were tested.

\section{Co-culture of hPCs and MSCs promotes chondrocyte proliferation independent of the MSCs origin}

EdU incorporation and cell tracking were used to investigate cell proliferation in the pellets. At day 2 after cell seeding EdU positive cells were detected in all conditions tested (figure. 2B). Quantitative data are shown in figure 2C and figure 2D. Percentages of EdU positive MSCs in co-culture pellets were close to that of $100 \%$ MSCs. No significant differences were observed. Percentages of proliferating hPCs in co-culture pellets were significantly higher as compared to $100 \% \mathrm{hPCs}$.

After 4 weeks of co-culture, ratios of cells derived from hPC or MSC donors were determined by STR analysis (figure. 2E, F and G). The distinguishable loci between hPC and MSC donors are illustrated, which varied between pairs of MSCs and hPCs. On average these loci showed a clear change in the ratio of MSCs and hPCs compared to the initial seeding density, irrespective of the source of MSCs. Three MSC donors were tested for each co-culture combination with essentially comparable results. 

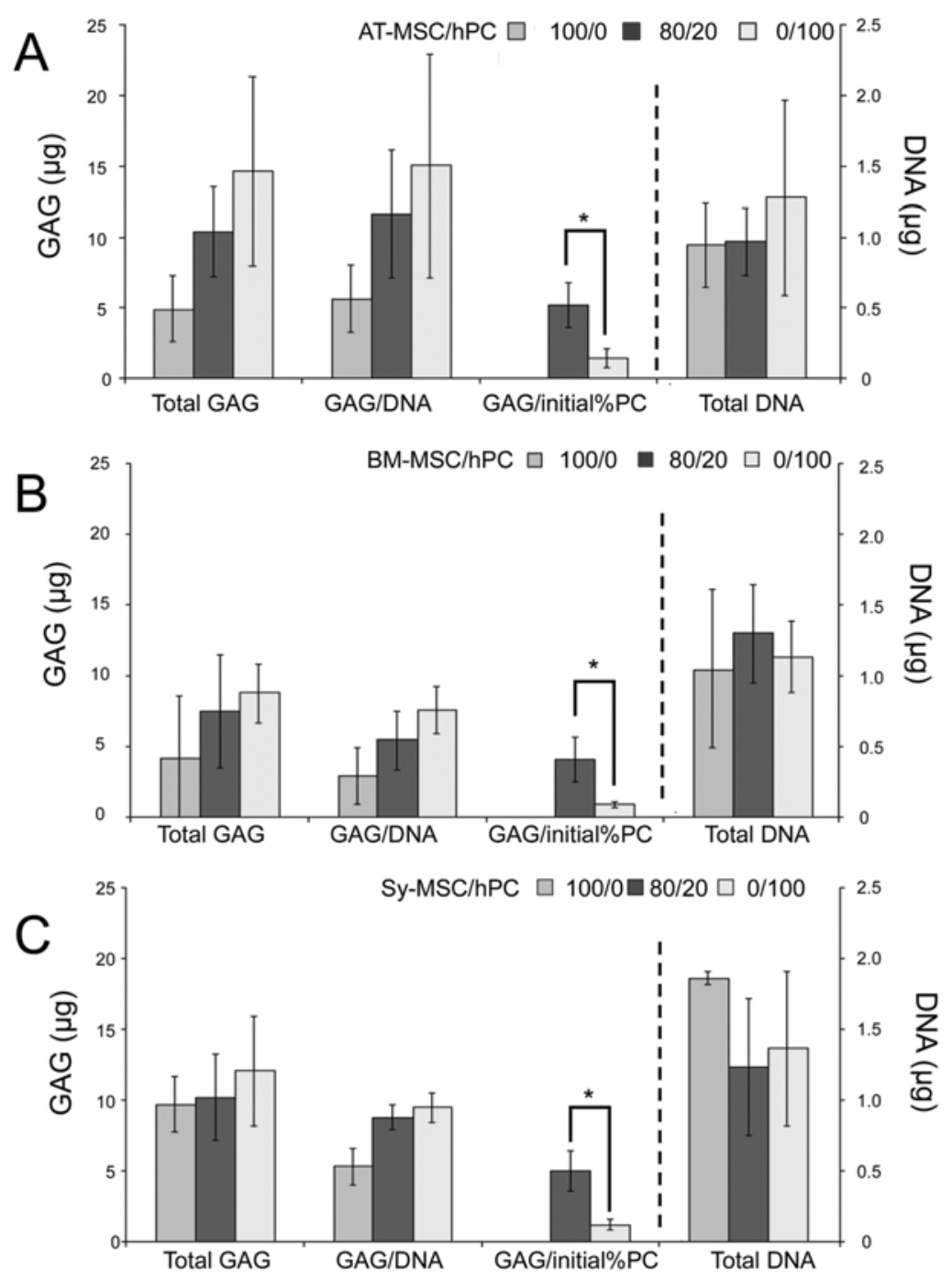

Figure 3 Trophic effects in co-culture are indepen-dent of the origin of the MSC. Human chondrocytes were co-cultured in pellets with AT-MSCs (A), BM-MSCs (B) or Sy-MSCs (C) in 3 different ratios in chondro-genic differentiation med-ium. GAG and DNA of cell pellets ( $\mathrm{n}=6$ per donor) were quantitatively mea-sureed 4 weeks after cul-ture. Ratios of MSCs and bPCs are indicated by the bar colors Scale on the left is for "Total GAG", "GAG/DNA" and "GAG/ initial \%PC", while scale on the right is for "DNA". Asterisk represents $P<0.05$. Data are the aver-age of 3 donors per cell source \pm S. D. 


\section{Discussion}

Multiple mechanisms have been postulated to explain increased cartilage matrix formation in co-culture pellets of MSCs and chondrocytes ${ }^{[2]}$. When this beneficial effect of co-culture was first discovered ${ }^{[3]}$, it was believed that chondrocytes stimulated the chondrogenic differentiation of MSCs, and that this contributed to the increased cartilage matrix formation in co-culture. Several studies supported this idea [3, 33-34]. It was shown that conditioned medium of chondrocytes induced osteochondrogenic differentiation of $\operatorname{MSCs}[33,35]$, and co-culturing with chondrocytes in pellets induced chondrogenic gene expression in MSCs[34]. Results from these experiments, however, were not conclusive on the role of each cell type in the coculture, since long term cell tracking was not performed. Previously, we have shown that increased cartilage formation in co-culture pellet is not due to chondrogenic differentiation of MSCs, but is predominantly caused by MSCs exerting a trophic effect on the chondrocytes stimulating cell proliferation and matrix deposition. The effect of MSCs on chondrocytes in pellet co-cultures can be discerned in various effects: 1) MSCs stimulate proliferation of chondrocytes at an early stage of the cell pellet cultures; 2) MSCs stimulate GAG formation and extracellular matrix production by chondrocytes in co-culture pellets; 3) As a consequence of the increased chondrocyte proliferation, the initial seeding ratio between chondrocytes and MSCs changes over time in favor of the chondrocytes; 4) This effect is further exaggerated by the preferential cell death of MSCs in the co-culture pellets. These findings were essentially confirmed in a recent publication of Acharya et al.[6]

Our previous study has raised a number of additional unresolved questions: First, is the trophic effect of the MSCs in co-culture dependent on the culture conditions? Our previous pellet co-cultures were performed in serum containing medium lacking chondrogenic factors that are essential for stimulating chondrogenic differentiation of MSCs in vitro. This raises the question whether the absence of MSC differentiation and the disappearance of MSCs over time in these co-culture experiments were the result of culture conditions that are unfavorable for MSCs. To address this issue we have repeated our co-culture experiments in medium that is supportive of chondrogenic differentiation of MSCs. We have performed the experiments with 3 donors that showed 


\section{Chapter 4}

capacity to differentiate into chondrocytes, albeit in a variable degree. Our results demonstrated that BM-MSCs essentially exerted a similar trophic role in the co-cultures irrespective of the culture conditions. Also in chondrogenic medium we did not observe overt differentiation of the MSCs in chondrocytes. Based on the results of the present study, it appears that there is a common mechanism in both culture conditions, although we do find some differences in the fate of MSCs in co-culture depending on the culture medium. The most notable difference is that the ratio change in chondrogenic differentiation medium is not as dramatic as that in proliferation medium. As we reported, MSCs mixed with bPCs almost disappeared in co-culture pellets in absence of chondrogenic factors, while around $40 \%$ of MSCs remained in co-culture pellets in differentiation medium [5]. This suggests that MSCs cultured in pellets can survive better in chondrogenic differentiation medium than in proliferation medium. We did not find experimental evidence that the better survival of the MSCs was due to stimulation of chondrocyte differentiation. In fact, the expression of human specific ACAN and COL2 after 4 weeks of culture was markedly reduced in the co-culture pellets compared to pellets of pure MSCs. In addition, the production of GAGs corrected for the initial seeding percentage of chondrocytes did not differ between co-culture pellets cultured in proliferation medium [5] or in chondrogenic medium (this study). Although we cannot conclude from these experiments that none of the MSCs had differentiated into chondrocytes, this effect appears to be marginal in both culture conditions. The most marked difference between both studies is that the ratio of MSCs dropped from $80 \%$ to below $5 \%$ after 4 weeks when pellets are co-cultured in proliferation medium, while only a reduction to $40 \%$ was seen in chondrogenic medium. Despite this difference, similarities in both models were more common. For instance, in both models a homogenous distribution of cartilage matrix compounds was found in the co-culture pellets even though the MSCs in co-culture pellets with chondrocytes preferentially end up in the center of the pellet, irrespective of the culture conditions [5].

The second question raised by our previous study is whether the observed role of the MSCs in the co-cultures is dependent on the source of MSCs. Our previous results were obtained with BM-MSCs. However, beneficial effects of co-culture for cartilage matrix formation was also found in combination of chondrocytes and a variety 
of other cell types, such as adipose-tissue derived stem cells, human embryonic stem cells, fibroblasts and meniscus cells [36-39]. It is still unclear whether MSCs derived from adipose tissue or synovium have a comparable trophic role in co-culture. To answer this question, we report that MSCs isolated from adipose tissue and synovial membrane had similar trophic effects and similar behavior as BM-MSCs in co-culture with chondrocytes. We show that MSCs irrespective of their origin stimulate chondrocyte proliferation and increase total GAG corrected for initial seeding percentage of chondrocytes to comparable extent. Furthermore, we show a similar decrease in the percentage of MSCs in pellet co-cultures over time. Given these remarkably similar observations, we concluded that the MSCs from various cell sources have likely exerted similar roles.

Adipose tissue has long been considered as an alternative to bone marrow as MSCs source, since it can be obtained relatively easily in large quantities with relatively low donor morbidity and contains a much higher frequency of MSCs, as compared to bone marrow [15, 30]. Piles of documents had pointed out the potential use of ATMSCs in a variety of tissue engineering applications [40-43]. Although less studies have been performed with synovium derived MSCs, the synovium has recently received attention as a promising cell source for cartilage tissue engineering [24, 31, 44]. Here, we report for the first time that MSCs isolated from bone marrow, adipose tissue and synovium behave similarly in co-culture pellets with MSCs by acting as trophic mediators stimulating chondrocyte proliferation and matrix production. Our data suggest that trophic effects of MSCs could be a general mechanism by which MSCs from different origins orchestrate tissue function repair.

Taken together, our results demonstrate that in co-culture pellets MSCs stimulate cartilage formation due to a trophic effect with chondrocytes rather than differentiating into chondrocytes, irrespective of the culture conditions or their origin. This implies that the trophic effect of MSCs in co-cultures is a general phenomenon which may have potential implications for the use of MSCs in cartilage repair strategies. 


\section{References}

1. Brittberg M, Lindahl A, Nilsson A, et al. Treatment of deep cartilage defects in the knee with autologous chondrocyte transplantation. N Engl J Med 1994;331:889-895.

2. Hendriks J, Riesle J, van Blitterswijk CA. Co-culture in cartilage tissue engineering. J Tissue Eng Regen Med 2007;1:170-178.

3. Tsuchiya K, Chen G, Ushida T, et al. The effect of coculture of chondrocytes with mesenchymal stem cells on their cartilaginous phenotype in vitro. Materials Science \& Engineering CBiomimetic and Supramolecular Systems 2004;24:6.

4. Gruber HE, Deepe R, Hoelscher GL, et al. Human adipose-derived mesenchymal stem cells: direction to a phenotype sharing similarities with the disc, gene expression profiling, and coculture with human annulus cells. Tissue Eng Part A 2010;16:2843-2860.

5. Wu L, Leijten JC, Georgi N, et al. Trophic effects of mesenchymal stem cells increase chondrocyte proliferation and matrix formation. Tissue Eng Part A 2011;17:1425-1436.

6. Acharya C, Adesida A, Zajac P, et al. Enhanced chondrocyte proliferation and mesenchymal stromal cells chondrogenesis in coculture pellets mediate improved cartilage formation. J Cell Physiol 2011.

7. Caplan AI, Dennis JE. Mesenchymal stem cells as trophic mediators. J Cell Biochem 2006;98:1076-1084.

8. Singer M. Trophic functions of the neuron. VI. Other trophic systems. Neurotrophic control of limb regeneration in the newt. Ann N Y Acad Sci 1974;228:308-322.

9. Bruder SP, Fink DJ, Caplan AI. Mesenchymal stem cells in bone development, bone repair, and skeletal regeneration therapy. J Cell Biochem 1994;56:283-294.

10. Kassis I, Vaknin-Dembinsky A, Karussis D. Bone marrow mesenchymal stem cells: agents of immunomodulation and neuroprotection. Curr Stem Cell Res Ther 2011;6:63-68.

11. Li Y, Chen J, Zhang CL, et al. Gliosis and brain remodeling after treatment of stroke in rats with marrow stromal cells. Glia 2005;49:407-417.

12. Sassoli C, Pini A, Mazzanti B, et al. Mesenchymal stromal cells affect cardiomyocyte growth through juxtacrine Notch-1/Jagged1 signaling and paracrine mechanisms: Clues for cardiac regeneration. J Mol Cell Cardiol 2011.

13. Tang YL, Zhao Q, Qin X, et al. Paracrine action enhances the effects of autologous mesenchymal stem cell transplantation on vascular regeneration in rat model of myocardial infarction. Ann Thorac Surg 2005;80:229-236; discussion 236-227.

14. Lin Y, Liu L, Li Z, et al. Pluripotency potential of human adipose-derived stem cells marked with exogenous green fluorescent protein. Mol Cell Biochem 2006;291:1-10.

15. Zuk PA, Zhu M, Mizuno H, et al. Multilineage cells from human adipose tissue: implications for cell-based therapies. Tissue Eng 2001;7:211-228.

16. De Bari C, Dell'Accio F, Tylzanowski P, et al. Multipotent mesenchymal stem cells from adult human synovial membrane. Arthritis Rheum 2001;44:1928-1942.

17. Vemuri MC, Chase LG, Rao MS. Mesenchymal stem cell assays and applications. Methods Mol Biol 2011;698:3-8.

18. Chen FH, Tuan RS. Mesenchymal stem cells in arthritic diseases. Arthritis Res Ther 2008;10:223.

19. Motaln H, Schichor C, Lah TT. Human mesenchymal stem cells and their use in cell-based therapies. Cancer 2010;116:2519-2530.

20. Balber AE. Concise review: aldehyde dehydrogenase bright stem and progenitor cell populations from normal tissues: characteristics, activities, and emerging uses in regenerative medicine. Stem Cells $2011 ; 29: 570-575$.

21. Lin Y, Tang W, Wu L, et al. Bone regeneration by BMP-2 enhanced adipose stem cells loading on alginate gel. Histochem Cell Biol 2008;129:203-210.

22. Wu L, Zhu F, Wu Y, et al. Dentin sialophosphoprotein-promoted mineralization and expression of odontogenic genes in adipose-derived stromal cells. Cells Tissues Organs 2008;187:103-112.

23. Wu L, Wu Y, Lin Y, et al. Osteogenic differentiation of adipose derived stem cells promoted by overexpression of osterix. Mol Cell Biochem 2007;301:83-92.

24. Lee JI, Sato M, Kim HW, et al. Transplantatation of scaffold-free spheroids composed of synovium-derived cells and chondrocytes for the treatment of cartilage defects of the knee. Eur Cell Mater 2011;22:275-290.

25. Xue G, He M, Zhao J, et al. Intravenous umbilical cord mesenchymal stem cell infusion for the treatment of combined malnutrition nonunion of the humerus and radial nerve injury. Regen Med 
2011;6:733-741.

26. Park J, Euhus DM, Scherer PE. Paracrine and Endocrine Effects of Adipose Tissue on Cancer Development and Progression. Endocr Rev 2011.

27. Bhang SH, Cho SW, La WG, et al. Angiogenesis in ischemic tissue produced by spheroid grafting of human adipose-derived stromal cells. Biomaterials 2011;32:2734-2747.

28. Hendriks J, Riesle J, Vanblitterswijk CA. Effect of stratified culture compared to confluent culture in monolayer on proliferation and differentiation of human articular chondrocytes. Tissue Eng 2006; 12:2397-2405.

29. Wu L, Cai X, Dong H, et al. Serum regulates adipogenesis of mesenchymal stem cells via MEK/ERK-dependent PPARgamma expression and phosphorylation. J Cell Mol Med 2010;14:922-932.

30. Jurgens WJ, Oedayrajsingh-Varma MJ, Helder MN, et al. Effect of tissue-harvesting site on yield of stem cells derived from adipose tissue: implications for cell-based therapies. Cell Tissue Res 2008;332:415-426.

31. Lee SY, Nakagawa T, Reddi AH. Mesenchymal progenitor cells derived from synovium and infrapatellar fat pad as a source for superficial zone cartilage tissue engineering: analysis of superficial zone protein/lubricin expression. Tissue Eng Part A 2010;16:317-325.

32. Livak KJ, Schmittgen TD. Analysis of relative gene expression data using real-time quantitative PCR and the 2(-Delta Delta C(T)) Method. Methods 2001;25:402-408.

33. Hwang NS, Varghese S, Puleo C, et al. Morphogenetic signals from chondrocytes promote chondrogenic and osteogenic differentiation of mesenchymal stem cells. J Cell Physiol 2007;212:281-284.

34. Vadala G, Studer RK, Sowa G, et al. Coculture of bone marrow mesenchymal stem cells and nucleus pulposus cells modulate gene expression profile without cell fusion. Spine (Phila Pa 1976) 2008;33:870-876.

35. Lee JS, Im GI. Influence of Chondrocytes on the Chondrogenic Differentiation of Adipose Stem Cells. Tissue Eng Part A 2010.

36. Bigdeli N, Karlsson C, Strehl R, et al. Coculture of human embryonic stem cells and human articular chondrocytes results in significantly altered phenotype and improved chondrogenic differentiation. Stem Cells 2009;27:1812-1821.

37. Gunja NJ, Athanasiou KA. Effects of co-cultures of meniscus cells and articular chondrocytes on PLLA scaffolds. Biotechnol Bioeng 2009;103:808-816.

38. Hildner F, Concaro S, Peterbauer A, et al. Human adipose-derived stem cells contribute to chondrogenesis in coculture with human articular chondrocytes. Tissue Eng Part A 2009;15:39613969.

39. Hendriks JAA, Miclea RL, Schotel R, et al. Primary chondrocytes enhance cartilage tissue formation upon co-culture with a range of cell types. Soft Matter 2010;6:5080-5088.

40. Witkowska-Zimny M, Walenko K. Stem cells from adipose tissue. Cell Mol Biol Lett 2011;16:236-257.

41. Hildner F, Albrecht C, Gabriel C, et al. State of the art and future perspectives of articular cartilage regeneration: a focus on adipose-derived stem cells and platelet-derived products. J Tissue Eng Regen Med 2011;5:e36-51.

42. Wilson A, Butler PE, Seifalian AM. Adipose-derived stem cells for clinical applications: a review. Cell Prolif 2011;44:86-98.

43. Cherubino M, Rubin JP, Miljkovic N, et al. Adipose-derived stem cells for wound healing applications. Ann Plast Surg 2011;66:210-215.

44. Mochizuki T, Muneta T, Sakaguchi Y, et al. Higher chondrogenic potential of fibrous synoviumand adipose synovium-derived cells compared with subcutaneous fat-derived cells: distinguishing properties of mesenchymal stem cells in humans. Arthritis Rheum 2006;54:843-853. 
Chapter 4 


\section{Chapter 5}

\section{Fibroblast Growth Factor -1 is a mesenchymal stem cell secreted factor stimulating chondrocyte proliferation in co-}

culture*

Ling $\mathrm{Wu}^{1,2}$, Jeroen Leijten ${ }^{2}$, Clemens A. van Blitterswijk ${ }^{1}$, Marcel Karperien ${ }^{2}$

1. Department of Tissue Regeneration, MIRA-Institute for Biomedical Technology and Technical Medicine, University of Twente, Enschede, 7522NB, the Netherlands.

2. Department of Developmental BioEngineering, MIRA-Institute for Biomedical Technology and Technical Medicine, University of Twente, Enschede, 7522 $\mathrm{NB}$, the Netherlands.

*Manuscript in preparation. 


\section{Abstract}

Previously, we have shown that mesenchymal stem cells (MSCs) in co-culture with primary chondrocytes secrete (a) soluble factor(s) that increase(s) chondrocyte proliferation. In this study we set out to identify this factor(s). Microarray experiments of human bone marrow derived MSCs (hMSCs) and primary chondrocytes (hPCs) in pellet monoculture or in co-culture revealed a number of candidate secreted soluble factors. Of these, Fibroblast Growth Factor-1 (FGF-1) mRNA expression was markedly increased in co-cultures predominantly due to up-regulated expression in MSCs as revealed by immunofluorescence combined with cell tracking and by species specific PCR in co-culture pellets of hMSCs and bovine PCs. ELISA demonstrated increased FGF-1 secretion in medium of pellet co-cultures. Blocking of FGF signaling in coculture pellets by specific FGF receptor inhibitors completely blocked chondrocyte proliferation. Neutralizing FGF-1 activity in MSC conditioned medium by anti-FGF-1 antibodies completely blocked chondrocyte proliferation also. In conclusion, we demonstrate that MSCs increase FGF-1 secretion upon co-culture with chondrocytes, which in turn is responsible for increased chondrocyte proliferation in pellet co-cultures. 


\section{Introduction}

Autologous chondrocytes implantation (ACI) is currently considered the golden standard for treating large size cartilage defects. It has been proposed that partial replacement of chondrocytes with mesenchymal stem cells (MSCs) can potentially circumvent the problem of chondrocyte expansion, which is required in current ACI protocols to obtain sufficient cells and is associated with loss of the chondrocyte phenotype [1]. Previous reports showed that cartilage matrix formation was increased in co-cultures of chondrocytes with MSCs compared to monocultures [2]. Further investigations revealed that the inductive effects of MSCs on cartilage formation were predominantly due to stimulation of chondrocyte proliferation in the co-culture pellets [3]. This effect was defined as a trophic effect of MSCs. Additional studies showed that this trophic effect of MSCs in chondrocyte co-cultures was independent of culture conditions and MSC cell sources [4].

Trophic effects of MSCs refer to more general observations in which MSCs produce factors influencing viability, proliferation, and matrix production of neighboring cells. So far, a trophic role of MSCs has been reported in many tissues other than cartilage. For example, when introduced either into stroked brain of rats MSCs promote functions of the neural system without differentiating themselves into neurons or any other supporting cells [5]. Likewise, it has also been reported that MSCs stimulate cardiomyocyte proliferation and vascular regeneration without differentiating into tissue-specific cells, both in vitro and in vivo [6-7].

Despite the rapid progress in recognizing the importance of the trophic role of MSCs in tissue regeneration, the underlying mechanism is not quite clear yet. MSCs secrete a number of growth factors and cytokines that potentially play a role in this effect. It was reported that conditioned medium (CM) of MSCs promoted wound healing in a scratch model in vitro by affecting cell migration and extracellular matrix formation, which suggests that the trophic activity of MSC may be mediated by secreted factors [8]. Moreover, CM of amniotic fluid derived MSCs accelerated wound healing in a mouse excision wound model. This data suggests that the MSC secreted factors are functional in vivo [9]. Also in our previous study, pellets of chondrocytes cultured for 1 week in MSCs conditioned proliferation medium showed a higher proliferation rate than 


\section{Chapter 5}

cells cultured in non-conditioned proliferation medium [3]. Meanwhile, higher proliferation rates as illustrated by increased EdU (an analog of BrdU) incorporation was associated with increased DNA contents as well as total GAG contents [3]. Nevertheless, publications pinpointing the trophic role to a specific factor are scarce [10-11].

In this study, we used microarray experiments to investigate the differentially expressed genes in co-culture pellets of MSCs and chondrocytes and mono-culture pellets of MSCs and chondrocytes separately. A number of secreted factors were found to be up-regulated in co-culture pellets. Further studies identified Fibroblast Growth Factor-1 (FGF-1) as a major growth factor secreted by MSCs that promotes the proliferation of chondrocytes in co-cultures of MSCs and chondrocytes.

\section{Materials and Methods}

\section{Cell culture and expansion}

The use of human material in this study has been approved by a local Medical Ethical Committee.

Macroscopically healthy looking cartilage was obtained from knee or hip biopsies of patients with end stage osteoarthritis undergoing total knee or hip replacement. Chondrocytes were isolated as previously described [12]. In short, cartilage was dissected from underlying bone and connective tissue and was digested for 20-22 $\mathrm{h}$ in collagenase type II (0.15\% Worthington) in DMEM supplemented with penicillin $(100 \mathrm{U} / \mathrm{mL})$ and streptomycin $(100 \mathrm{mg} / \mathrm{mL})$. Human primary chondrocytes (hPCs) were then cultured in chondrocyte proliferation medium (DMEM supplemented with $10 \%$ FBS, $1 \times$ non-essential amino acids, $0.2 \mathrm{mM}$ Ascorbic acid 2-phosphate (AsAP), $0.4 \mathrm{mM}$ proline, $100 \mathrm{U} / \mathrm{ml}$ penicillin and $100 \mu \mathrm{g} / \mathrm{ml}$ streptomycin). More details about chondrocyte isolation are described elsewhere [13]. Bovine primary chondrocytes (bPCs) were isolated from full-thickness cartilage of knee biopsies from cows of approximately 6 months old with the same protocol as human chondrocytes. Bone marrow mesenchymal stem cells (BM-MSCs) were separated from human bone marrow aspirates as described previously [14]. Briefly, 100ul of bone marrow aspirate was mixed with $900 \mu \mathrm{l}$ red cell lysing buffer for 5-10 minutes on ice. Then the mononuclear cell fraction was counted. Whole bone marrow was plated at a density of 50000 
cells $/ \mathrm{cm}^{2}$ in culture flasks in MSC proliferation medium ( $\alpha$-MEM, supplemented with $10 \%$ fetal bovine serum, $1 \%$ L-glutamin, $0.2 \mathrm{mM}$ ascorbic acid, $100 \mathrm{U} / \mathrm{ml}$ penicillin, 10 $\mu \mathrm{g} / \mathrm{ml}$ streptomycin and $1 \mathrm{ng} / \mathrm{ml} \mathrm{bFGF}$ ), plus $1 \%$ heparin. Medium was refreshed every 3-4 days until confluence. All reagents used for cell culture were purchased from Gibco, Invitrogen (Paisley, UK), unless otherwise stated. Common chemicals were purchased from Sigma-Aldrich.

\section{Cell tracking with organic fluorescent dyes}

The organic fluorescent dye, CM-DiI (Molecular Probes, Eugene, OR) was used for cell tracking in co-cultures. Cells were labeled according to the manufacturer's protocol. Briefly, cells were trypsinized and resuspended in PBS at a concentration of 2 $\times 10^{6}$ cells $/ \mathrm{ml}$. Cells were incubated with dye (final concentration of $4 \mu \mathrm{M}$ ) at $37^{\circ} \mathrm{C}$ for 5 minutes followed by treatment at $4^{\circ} \mathrm{C}$ for 15 minutes. Cells were washed with PBS and applied in co-culture experiments.

\section{Co-culture of hPCs and hMSCs}

For co-culture of hPCs or bPCs and hMSCs, 40000 chondrocytes and 160000 hMSC (ratio of PC:hMSCs=1:4) were seeded in one well of a round bottom ultralow attachment 96 wells plate in chondrocyte proliferation medium and centrifuged for 3 min at 2000rpm. Medium was refreshed twice a week. Cell pellets were cultured for 2 days before RNA isolation and EdU staining.

\section{EdU labeling and staining}

Cell proliferation in pellets was examined with the Click-iT ${ }^{\circledR}$ EdU Imaging Kit (Molecular Probes, Eugene, OR). Cell pellets were cultured in chondrocyte proliferation medium. EdU (5-ethynyl-2'-deoxyuridine) was added to the culture media at a concentration of $10 \mu \mathrm{M}, 24$ hours before harvesting the samples. Cell pellets were then washed with PBS and fixed with $10 \%$ formalin for $15 \mathrm{~min}$. Samples were embedded in cryomatrix, and cut into $10 \mu \mathrm{M}$ sections with a cryotome (Shandon, France). Sections were permeabilized and stained for EdU with Alexa 488 cocktail. Nuclei were counterstained with Hoechst 33342.

\section{RNA isolation and quantitative PCR}

Gene expression analysis was performed as described before [15]. In short, RNA samples of pellets were isolated with the NucleoSpin ${ }^{\circledR}$ RNA II Kit (Macherey- 


\section{Chapter 5}

Nagel, Düren, Germany). For quantitative (q)PCR, one microgram of total RNA was reverse-transcribed into cDNA using the iScript cDNA Synthesis kit (Bio-Rad, Hercules, CA). qPCR was performed on cDNA samples by using the iQ SYBR Green Supermix (Bio-Rad, Hercules, CA). PCR Reactions were carried out on MyiQ2 Two-Color RealTime PCR Detection System (Bio-Rad, Hercules, CA) under the following conditions: cDNA was preheated for $15 \mathrm{~min}$ at $95{ }^{\circ} \mathrm{C}$, denatured for $5 \mathrm{~min}$ at $95{ }^{\circ} \mathrm{C}$, followed by 45 cycles, consisting $15 \mathrm{~s}$ at $95^{\circ} \mathrm{C}, 15 \mathrm{~s} 60^{\circ} \mathrm{C}$ and $30 \mathrm{~s}$ at $72^{\circ} \mathrm{C}$. For each reaction a melting curve was generated to test primer dimer formation and non-specific priming. The primers for real-time PCR are listed in supplementary table 1 and table 2. Calculation of Relative Expression was performed with Bio-Rad iQ5 optical system software (version 2.0) using the double delta Ct method [16]. GAPDH was used for normalization.

\section{Microarray processing and statistical analysis}

For microarray study, equal cell numbers of $3 \mathrm{hPCs}$ donors were pooled. The pooled cell fraction was used in pellet monocultures $(n=3)$ and in co-cultures with 3 distinct donors of hMSCs. RNA was also isolated from the 3 MSC donors cultured in pellets individually. RNA samples were prepared as described in the previous section. NuGEN Ovation PicoSL WTA System kit followed by Encore BiotinIL module was used to generate biotinylated sscDNA starting from $50 \mathrm{ng}$ total RNA. $750 \mathrm{ng}$ of the obtained samples was hybridized onto Illumina HumanHT-12 v4 Expression BeadChips. Samples were scanned using the Illumina iScan array scanner. Gene expression profiling was performed using Illumina's Genomestudio v. 2010.3 software with the default settings advised by Illumina. The raw fluorescence intensity values were normalized applying quantile normalization. Differential gene expression was analysed using the commercial software package Genespring, version 11.5.1. (Agilent Technologies). Genes with at least a twenty percent difference between observed and expected values were selected and tested for significance using a one way ANOVA with a Benjamini-Hochberg FDR correction and Tukey HSD post hoc test using a cut-off rate of $\mathrm{P} \leq 0.05$. The Expected value of gene expression was defined as $80 \%$ of the expression of the gene in monoculture pellets of hMSCs added to $20 \%$ of the expression of the gene in monoculture pellets of hPC both determined by microarray analysis assuming that gene expression was not influenced by the co-culture. The Observed 
values of gene expression reflected the actually expression level of one gene measured in the microarray experiment (Supplementary Fig. S1). Changes in gene expression in annotated canonical pathways and bio-functions were visualized using ingenuity pathway analysis software (Ingenuity Systems). Search Tool for the Retrieval of Interacting Genes/Proteins (STRING) was used to investigate the predicted interaction network [17]. Clusters were formed using a k-means clustering algorithm.

\section{ELISA assay}

The concentrations of human FGF-1 in the conditioned medium of co-culture pellets or hMSC mono-cultures were determined by a human FGF-1 ELISA kit (R\&D system, Oxon, UK) according to the manufacturer's instructions. Absorbance was measured on an ELISA plate reader at $450 \mathrm{~nm}$ and $550 \mathrm{~nm}$. The $450 \mathrm{~nm}$ values were subtracted by the $550 \mathrm{~nm}$ values for correction of the optical imperfections in the microplates.

\section{Immunofluorescent staining}

At day 2 after seeding, co-culture pellets were harvested for immunofluorescent staining. Cell pellets were washed with PBS and fixed with 10\% formalin for $15 \mathrm{~min}$. Samples were then embedded in cryomatrix, and cut into $10 \mu \mathrm{M}$ sections with a cryotome (Shandon, France). Sections were permeabilized and blocked in PBS containing $0.5 \%$ Triton-X 100 and $0.5 \%$ bovine serum albumin (BSA) for 15 min at room temperature. Slides were subsequently incubated overnight at $4^{\circ} \mathrm{C}$ with a goat polyclonal antibody against FGF-1 (R\&D system, Oxon, UK). Subsequently, slides were incubated with a secondary antibody conjugated to Alexa 488 (Molecular Probes, Eugene, OR), and nuclei were counterstained with 4,6-diamidino-2-phenylindole (DAPI; Molecular Probes, Eugene, OR).

\section{Image acquisition and analysis}

All fluorescent images were taken with a BD pathway 435 confocal microscope (BD Biosciences, Rockville, MD). Using montague capture, images of high resolutions were obtained covering the entire section of a pellet. Separated images were captured at the green channel (Alexa 488), red channel (DiI) and blue channel (Hoechst 33342). ImageJ software [18] was used for cell counting. Briefly, we first set a threshold to avoid artifacts manually. Then we counted the number of green cells, red 
cells, green + red cells, and total cells by running plug-ins written with macro language of ImageJ (available on request). Values represent the mean + /- standard error of at least 3 biological replicates.

\section{Collection of conditioned medium}

To obtain conditioned medium, DMEM was incubated with hMSCs cultured in monolayer at $90 \%$ confluency for $48 \mathrm{~h}$, passed through a $0.22 \mathrm{~mm}$ filter, and stored in $20{ }^{\circ} \mathrm{C}$ until use. Upon usage, conditioned medium was thawed, put in Amicon Ultra-15 Centrifugal Filter Units (Millipore, Billerica, MA) with a cut off of 3000 dalton Nominal Molecular Weight Limit, and centrifuged at $4000 \times \mathrm{g}$ for 40 minutes. The concentrated solute (still named conditioned medium) was used to supplement chondrocyte proliferation medium containing FBS and used to culture pellets of 200 000 hPCs.

\section{Statistical analysis:}

Differences between culture conditions of MSCs and hPCs were examined for statistical significance with one-way analysis of variance (ANOVA) followed by Tukey HSD Test. Comparisons between hMSCs and hPCs in the same conditions were made by using the Student's test. $P$ values of $<0.05$ were considered as significant.

\section{Results}

\section{Co-culture enhances proliferation of hPCs}

Previously, we reported that proliferation of chondrocytes was enhanced in a xenogenic co-culture system of bovine chondrocytes and hMSCs. In this study, we examined proliferation of hPCs in a fully human co-culture system [3]. Since the bPC proliferated most at day 2 after establishing the co-culture, we analyzed proliferation in fully human co-culture pellets at day 2 by measuring EdU incorporation. To distinguish hMSCs from hPC, the latter cells were labeled with the membrane bound fluorescent tracer CM-DiI (red). As shown in Fig. 1A, EdU positive cells were predominantly found at the periphery of the cell pellets in which the red labeled hPCs resided. The percentage of EdU positive hPCs and EdU positive hMSCs in the co-cultures was determined. Our results showed that co-culture significantly stimulated EdU incorporation in hPCs $(\mathrm{p}<0.01)$ but not in hMSCs (Fig. 1B). 


\section{Microarray study identifies a group of genes regulated by the interactions between MSCs and hPCs}

Since after 48 hours in co-culture, proliferation of chondrocytes was strongly increased we chose this time point for microarray analysis to identify genes that are regulated by the interaction between MSCs and hPC. Total RNA was isolated from 3 independent experiments each with 3 experimental conditions: monoculture pellet of MSCs, co-culture pellet of MSCs and hPC (ratio 4:1) and monoculture pellets of hPC. Each RNA sample was hybridized to (Illumina HumanHT-12 v4 Expression BeadChips). After data normalization, the ratio between Observed gene expression as determined by the microarray experiment and Expected values of gene expression was calculated (supplementary figure $\mathrm{S} 1$ ) and significantly changed genes $(\mathrm{P} \leq 0.05$, after one way ANOVA with a Benjamini-Hochberg FDR correction and Tukey HSD post hoc test) deviating more than $20 \%$ from the Expected value were selected. Hierarchical cluster analysis using the significantly changed genes showed a higher degree of similarity in gene expression profile with hMSC mono-culture pellets than with hPCs mono-culture pellets (Fig. 1C). Fig. 1D shows a global comparison between Observed values and Expected values of each gene analyzed in the microarray experiment in coculture pellets. Using a threshold for up-regulated genes of Ratio $_{\mathrm{O} / \mathrm{E}}>1.2$ and a threshold for down-regulated genes of Ratio $_{\mathrm{O} / \mathrm{E}}<0.8$, the expression of 180 genes was positively regulated by co-culture while the expression of 93 genes was negatively regulated by co-culture respectively (supplementary Table 3 for a full list of these differentially regulated genes). The expression of the majority of genes (22835) was not influenced by the co-culture and felt in the region between the two thresholds (grey points). qPCR analysis of 12 up-regulated and 5 down-regulated genes confirmed the direction of change in gene expression based on the Ratio ${ }_{\mathrm{O} / \mathrm{E}}$. The ratios determined by qPCR were on average slightly higher or lower for up-regulated and down-regulated genes, respectively (Fig. 1E). The list of 180 up-regulated genes was then imported into ingenuity pathway analysis software to examine whether the changes in gene expression could be annotated to canonical pathways and bio-functions. Signaling pathways, such as cellular growth and proliferation in molecular and cellular functions pathways (Fig. S2), cyclins and cell cycle regulation in canonical pathways (Fig. S3) and skeletal and 


\section{Chapter 5}
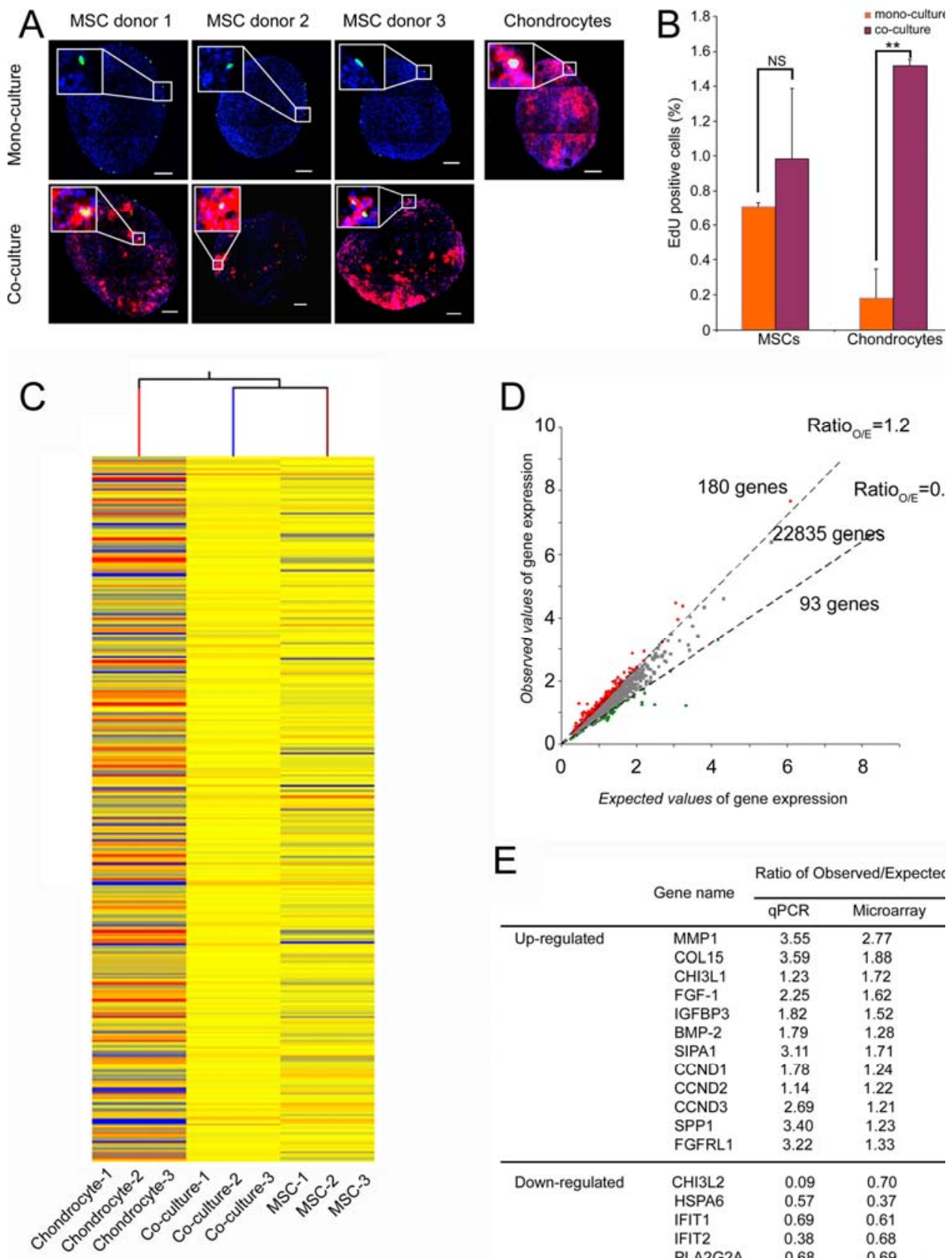

\begin{tabular}{llcc}
\multirow{2}{*}{ E } & & & \\
& & & \\
& & & \\
& Gatio of Observed/Expecter \\
\cline { 3 - 4 } & & qPCR & Microarray \\
\cline { 3 - 4 } Up-regulated & MMP1 & 3.55 & 2.77 \\
& COL15 & 3.59 & 1.88 \\
& CHI3L1 & 1.23 & 1.72 \\
& FGF-1 & 2.25 & 1.62 \\
& IGFBP3 & 1.82 & 1.52 \\
& BMP-2 & 1.79 & 1.28 \\
& SIPA1 & 3.11 & 1.71 \\
& CCND1 & 1.78 & 1.24 \\
& CCND2 & 1.14 & 1.22 \\
& CCND3 & 2.69 & 1.21 \\
& SPP1 & 3.40 & 1.23 \\
& FGFRL1 & 3.22 & 1.33 \\
\hline \multirow{2}{*}{ Down-regulated } & CHI3L2 & 0.09 & 0.70 \\
& HSPA6 & 0.57 & 0.37 \\
& IFIT1 & 0.69 & 0.61 \\
& IFIT2 & 0.38 & 0.68 \\
& PLA2G2A & 0.68 & 0.69
\end{tabular}


Figure 1 Co-culture increases proliferation of human primary chondrocytes; and global gene expression analysis in co-culture pellets. (A) EdU staining of pellets at day 2. Chondrocytes were labeled with CM-DiI (red). EdU incorporation into newly synthesized DNA was visualized by Alexa 488 (green). Double labeled cells indicate EdU positive chondrocytes. Nuclei were counterstained with Hoechst 33342 (blue). A representative picture of each condition is shown. The upper panel shows overviews of pellets, while the lower panel shows magnified pictures of the boxed area. Scale bar=100 $\mu \mathrm{m}$. (B) Quantification of EdU positive cells in panel A in MSCs and hPC using cell tracking with CM-Dil. Data represents the mean EdU positive MSCs of 3 donors and hPC in monoculture or in co-culture (ratio MSC vs hPC: 4:1) each measured in at least triplicate. Values shown are mean $+/-$ standard deviation (S.D.). P values were calculated by student's t-test. $* *=P<0.01$. (C) RNA samples were isolated at 48 hours and applied to microarray study. Hierarchical clustering analysis shows the comparison of gene expression profiles in mono-cultures and cocultures. The gene expression pattern in co-cultures resembles more the gene expression patterns in MSCs than in hPCs. (D) Observed gene expression levels as determined by microarray analysis in co-cultures of hMSCs and hChondrocytes are plotted against expected gene expression levels defined as $80 \%$ of the expression of the respective gene in MSC monocultures added to $20 \%$ expression of the gene in chondrocyte monocultures. Dashed lines indicate thresholds for up- $(>1.2$ fold $)$ and down-regulated gene expression $(<0.8$ fold) compared to the calculated expected values. In red genes are depicted with deviate $>1.2$ fold from the expected ratio. In green are genes depicted that deviate $<0.8$ fold from the expected ratio. (E) Expression levels of differentially regulated genes in mono-cultures and co-cultures pellets were validated by qPCR. The expression values obtained from qPCR were also calculated for the Ratio of Observed values and Expected values.

muscular system development in physiological system development/function pathways (Fig. S4) were significantly changed. Activation of these signaling pathways was in line with our observations of increased proliferation and matrix formation in co-cultures. The genes analyzed by ingenuity pathway analysis software were listed in supplementary table 4. We next analyzed the differentially expressed genes using the Search Tool for the Retrieval of Interacting Genes/Proteins to investigate possible interaction networks of genes/proteins (Figure 2A). Four main clusters of interacting genes were identified. Two of these clusters were related to extracellular matrix and were centered on COMP (red cluster) and COL4A1 (green cluster), respectively. In line with increased EdU incorporation in co-culture pellets, 1 cluster contained intracellular cell cycle regulators, like CCND1, -2 and -3 . The $4^{\text {th }}$ cluster contained two secreted growth factors FGF-1 and BMP-2, which both are established modulators of chondrocyte proliferation and/or matrix production [19-21]. 

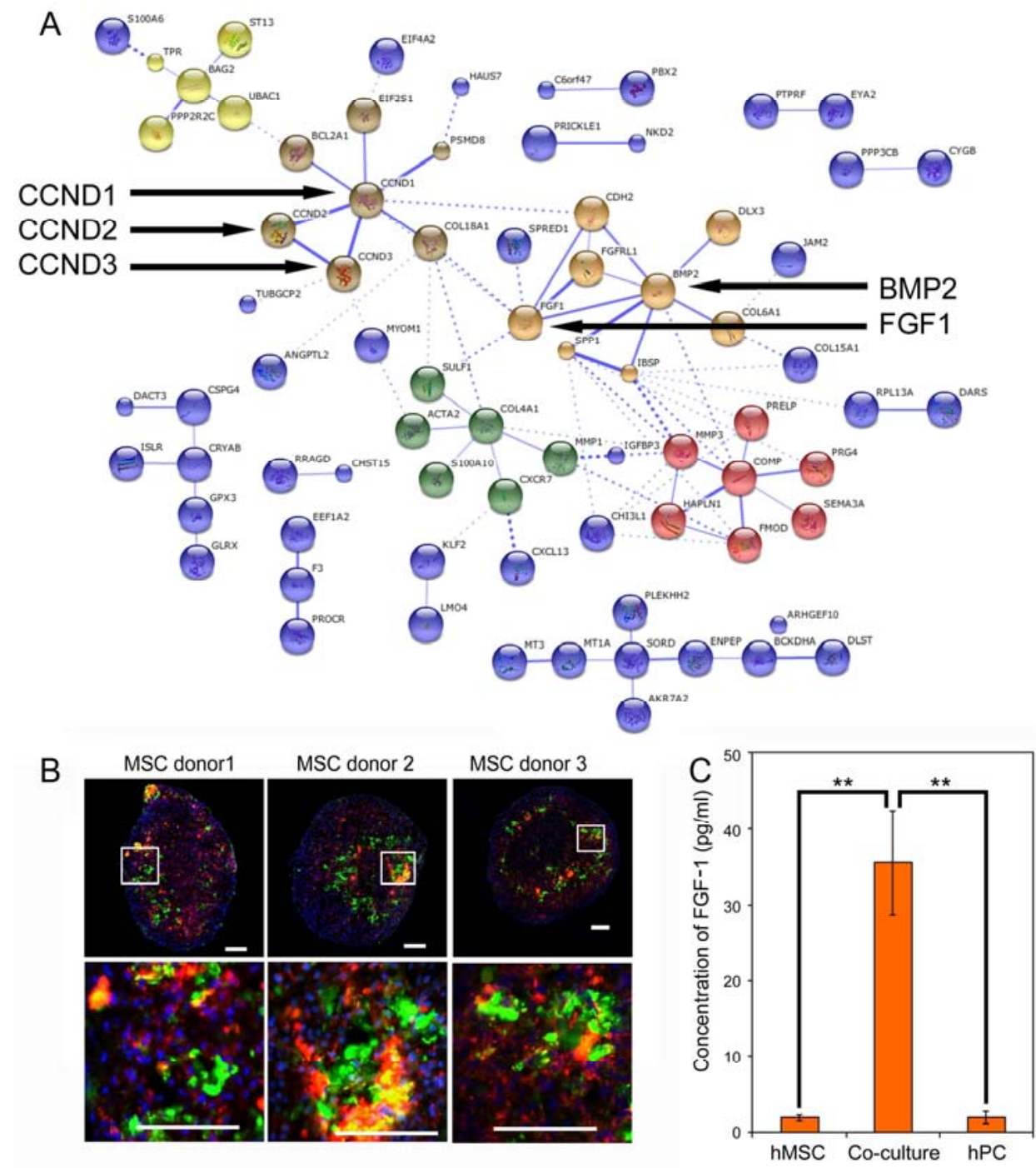

\begin{tabular}{ccccccc}
\hline & $\begin{array}{c}\text { Total cell } \\
\text { number }\end{array}$ & $\begin{array}{c}\text { Total hPC } \\
\text { number }\end{array}$ & $\begin{array}{c}\text { Labeling } \\
\text { efficiency (\%) }\end{array}$ & $\begin{array}{c}\text { FGF-1 positive } \\
\text { area (pixels) }\end{array}$ & $\begin{array}{c}\text { FGF-1 positive } \\
\text { area in hPC (pixels) }\end{array}$ & $\begin{array}{c}\text { \% of FGF positive area } \\
\text { in MSCs }\end{array}$ \\
\hline Donor 1 & 1529 & 265 & 19.72 & $3.4 \mathrm{E}+05$ & $9.2 \mathrm{E}+04$ & 73 \\
Donor 2 & 1737 & 295 & 16.61 & $5.1 \mathrm{E}+05$ & $1.4 \mathrm{E}+05$ & 72 \\
Donor 3 & 1738 & 306 & 18.54 & $3.1 \mathrm{E}+05$ & $8.7 \mathrm{E}+04$ & 72 \\
\hline Average & $1669 \pm 98$ & $289 \pm 17$ & $18.29 \pm 1.29$ & $3.9 \mathrm{E}+05 \pm 8.7 \mathrm{E}+04$ & $1.1 \mathrm{E}+05 \pm 2.5 \mathrm{E}+04$ & $72 \pm 0.28$
\end{tabular}


Figure 2 Interaction networks of genes/proteins of up-regulated genes; and immunofluorescent staining of FGF-1 in co-culture pellets. (A)The predicted interaction networks of the 180 up-regulated genes/proteins were studied by the Search Tool for the Retrieval of Interacting Genes/Proteins as described in materials and methods. (B) Human chondrocytes (labeled with the red fluorescent dye CM-DiI) were cocultured with MSCs for 2 days in chondrocyte proliferation medium in a 1:4 ratio. Immunofluorescent staining of FGF-1 (green) was performed on mid-saggital cryo-sections, while nuclei were counterstained with dapi. Pictures show a representative section from each donor. Double labeled cells are stained in yellow and represent FGF-1 positive chondrocytes. Lower panels are magnifications of the boxed areas. Scale bar=50 $\mu \mathrm{m}$. (C) Conditioned medium of co-culture and mono-culture pellets was collected at day 2 after seeding. Concentrations of FGF-1 were quantified by ELISA kit. Data represent the mean of 3 donors +/- SD. Statistical analysis was performed using one way ANOVA. $* *=p<0.01$. (D) Quantification of FGF-1 positively stained area. For each donor, three pellets were analyzed. For each pellet, at least six sections were analyzed. Average values +/- S.D. are given. Total cell numbers, number of hPC, FGF-1 positive area (pixels), and FGF-1 positive area in hPC (pixels) were counted as described in Materials and Methods. Labeling efficiency was calculated by dividing numbers of hPCs by total cell numbers. The \% of FGF positive area in MSCs was calculated as follows: $100 \% *((\mathrm{FGF}-1$ postive area in chondrocytes)/ FGF-1 positive area).

\section{FGF-1 expression and secretion is up-regulated in co-culture pellets}

Since pellet co-culture with MSCs also increases chondrocyte proliferation in a xenogenic co-culture model of hMSCs and bovine (b)PC, we studied differential expression of a selected set of genes in this model. By using species specific PCR it was possible to attribute the change in gene expression to either one of the two cell types. Most of the tested bovine genes, including BMP-2 and $F G F-1$, were expressed at higher levels in co-culture pellets than in bPC mono-cultures (Fig. 3A). Human specific qPCR, demonstrated a slight trend to increased expression of most genes in co-culture pellets compared with hMSCs mono-culture pellets (Fig. 3B). To quantify gene expression in each of the two cell types in co-culture pellets, we next used cross-species GAPDH for normalization. As shown in Fig. 3C, FGF-1 and CCNDI were the only two genes predominantly expressed by hMSCs. Since previously it has been shown that chondrocyte proliferation in co-culture pellets is stimulated by an MSC secreted factor [3] and CCND1 is an intracellular regulator, FGF-1 was selected for further experimentation. We then examined the expression of FGF-1 in co-culture pellets of hMSCs and hPC (4:1 ratio) in which the pPC were labeled red and FGF-1 was stained in green (Fig. 2B). FGF-1 staining resided predominantly in a ring in the periphery of 


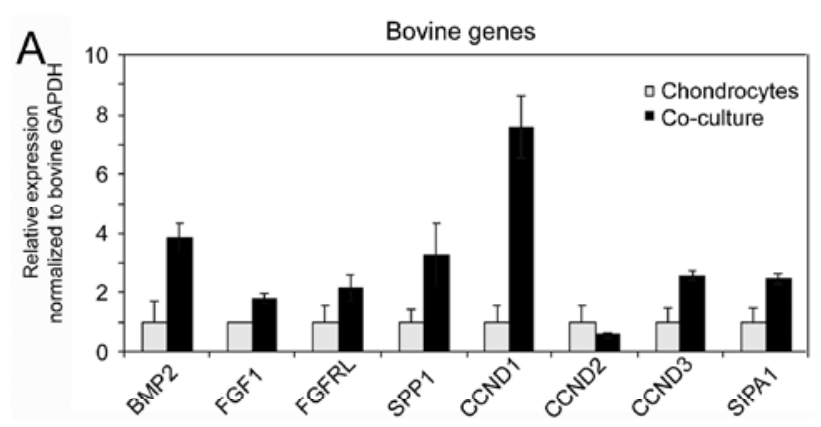

B
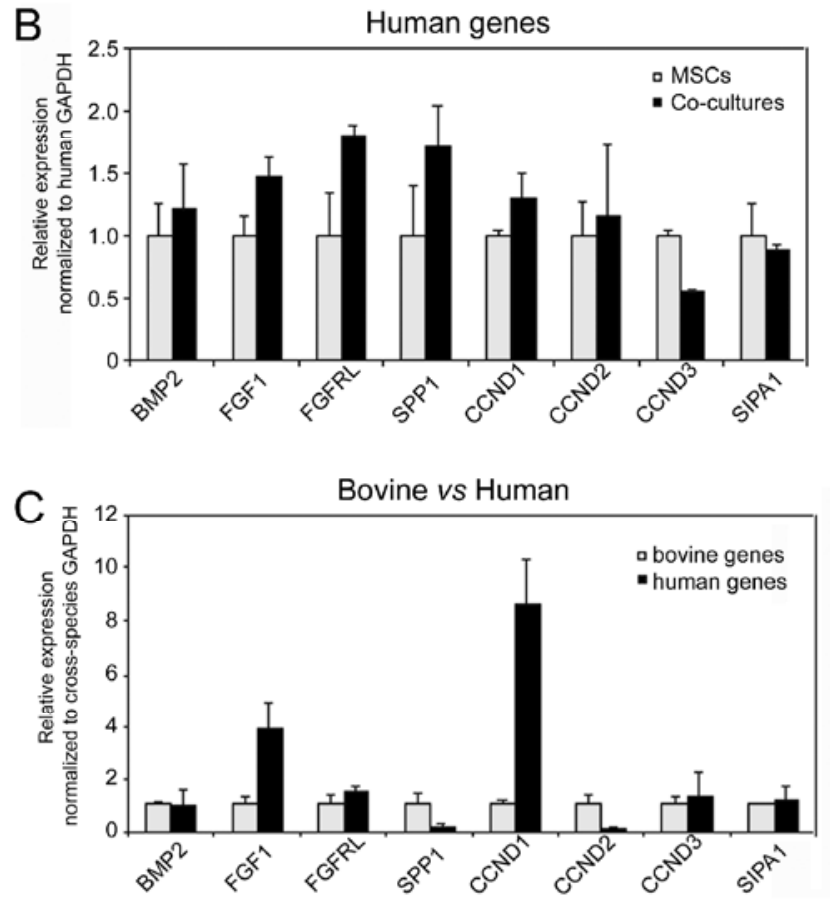

Figure 3 Species specific qPCR on xenogenic co-culture of bPCs and hMSCs (A) $80 \%$ of human MSCs and 20\% bovine chondrocytes were co-cultured for 2 days. RNA was then isolated. Expression of bovine genes was examined by real time RT-PCR using bovine specific primers which did not cross-react with human genes. Data are expressed as fold change relative to the expression in chondrocyte monocultures and represent the mean of 3 independent experiments $+/-$ S.D. (B) Expression of human genes was also demonstrated by real time RT-PCR using human specific primers which did not cross-react with bovine genes. Data are expressed as fold change relative to the expression in MSC monocultures and represent the mean of 3 independent experiments +/- S.D. (C) Expression levels of both bovine and human genes in co-culture pellets were normalized to cross-species GAPDH. Human gene expression is plotted relative to the expression of the respective bovine gene, which was set to 1 , and represent the mean of 3 independent experiments +/- S.D. 
the cell pellets in which also the hPCs resided. Overlay of fluorescent images demonstrated a few chondrocytes staining positive for FGF-1 but most FGF-1 staining was found in non-labeled MSCs. This was confirmed by quantitative analysis of the fluorescent images (Fig. 2D). In agreement with the seeding ratio of the labeled hPCs and hMSCs $18,3+/-1,3 \%$ of the counted cells were labeled red. On average $72 \%$ of the area stained for FGF-1 coincided with non-labeled MSCs, identifying the MSCs as the most likely predominant source of FGF-1 expression in co-culture pellets. This was in line with the mRNA expression data presented in Fig. 3. Remarkably, MSCs staining positive for FGF-1 were predominantly found in close vicinity of red labeled hPCs while staining in more distant MSCs was considerably lower or absent, providing support for the notion that the interaction between the hPCs and hMSCs increased FGF1 expression in the latter cells. In agreement with increased $F G F-1$ mRNA expression in co-culture pellets, conditioned medium of co-culture pellets, but not of mono-culture pellets contained considerable levels of $F G F-1$ (Fig. 2C).

\section{Blocking of the FGF signaling pathway inhibits proliferation of hPCs in co-culture pellets}

To study the role of increased FGF-1 expression and secretion in co-culture pellets, two small molecules (PD166866 and PD173074), blocking FGF receptor activation were added to the culture medium (Fig. 4A). EdU assays were performed to investigate the proliferation of cells in co-culture pellets. Quantification of EdU positive cells indicated that both inhibitors decreased the proliferation of both MSCs (Fig. 4B) and hPCs in co-culture pellets and nullified the increased chondrocyte proliferation normally found in co-culture pellets (Fig. 4C). To analyze the role of FGF-1 more specifically, a neutralizing antibody was used to block FGF-1 activity. FGF-1 neutralizing antibodies significantly reduced proliferation of hMSCs (Fig. 4B) and hPC (Fig. 4C) also in co-culture pellets.

\section{FGF-1 in MSC conditioned medium induces chondrocyte proliferation.}

Previously, we reported that secreted factors from conditioned medium of MSCs cultured in monolayer increased the proliferation of chondrocytes in pellet culture. The conditioned medium contained FGF-1 as determined by ELISA (Fig. 4D). The neutralizing antibody effectively blocked the activity of FGF-1. Then we performed 
Chapter 5

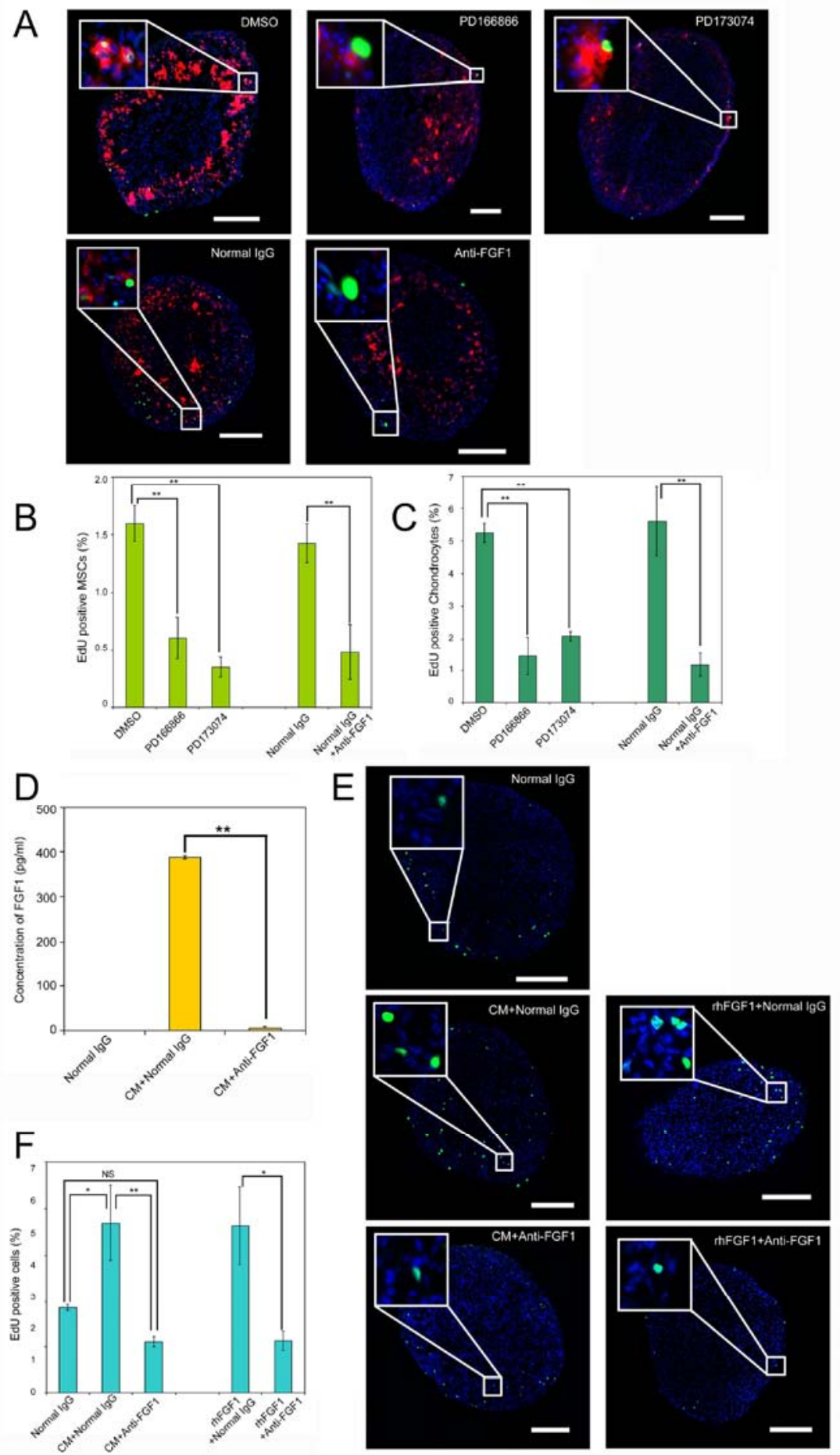


Figure 4 Inhibition of FGF-1 signaling reduces proliferation of chondrocytes in co-culture; and MSC secreted FGF-1 promotes chondrocyte proliferation. (A) EdU staining of co-culture pellets at day 2. Chondrocytes were labeled with CM-DiI (red). EdU incorporation into newly synthesized DNA was visualized by Alexa 488 (green). Nuclei were counterstained with Hoechst 33342 (blue). FGF-1 signaling was inhibited either by specific FGF-receptor inhibitors (500nM of PD166866 and 500nM of PD173074) or an FGF-1 neutralizing antibody (5 $\mu \mathrm{g} / \mathrm{ml})$. DMSO was used as vehicle for chemical inhibitors, while normal goat $\operatorname{IgG}(5 \mu \mathrm{g} / \mathrm{ml})$ served as control for neutralizing antibodies. Three donor pairs of MSCs and chondrocytes were analyzed. A

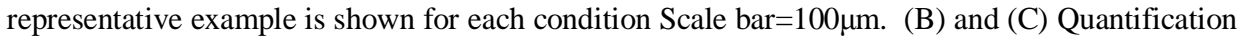
of EdU positive MSCs (B) or hPCs (C) in co-culture pellets. Data represent the average from 3 MSC donors, each measured in triplicate $+/$ - S.D. $* *=P<0.01$. (D) The concentration of FGF-1 in the conditioned medium (CM) of hMSCs cultured in monolayer was quantified by ELISA. CM was collected from three hMSCs donors. Medium was pooled for ELISA and EdU assays. (E) EdU staining was performed at day 2 to show proliferating cells in pellets of chondrocytes cultured in MSC conditioned medium. EdU incorporation into newly synthesized DNA was visualized by Alexa 488 (green). Nuclei were counterstained with Hoechst 33342 (blue). Activity of FGF-1 was blocked by a neutralizing antibody $(5 \mu \mathrm{g} / \mathrm{ml})$. Normal $\mathrm{IgG}(5 \mu \mathrm{g} / \mathrm{ml})$ and rhFGF-1 $(10 \mathrm{ng} / \mathrm{ml})$ served as a negative and positive control, respectively. Scale bar=100 $\mu \mathrm{m}$. (F) Quantification of EdU positive chondrocytes. Data represent the mean of 3 chondrocyte donors + - S.D.. $P$ values were calculated with student's t-test. $*=P<0.05$. $* *=P<0.01$. NS=non significance.

an EdU assay to test if FGF-1 contributed to chondrocyte proliferation. As shown in figure $4 \mathrm{E}$ and $\mathrm{F}$, pellets of hPCs cultured for 2 days in hMSCs conditioned medium $(\mathrm{CM}+$ Normal $\mathrm{IgG})$ showed more EdU incorporation than pellets cultured in nonconditioned proliferation medium (Normal $\mathrm{IgG}$ ) in line with previous observations [3]. Increased chondrocyte proliferation was reversed by adding anti-FGF-1 to the conditioned medium. Chondrocyte pellets cultured in proliferation medium containing human recombinant FGF-1 (10ng/ml rhFGF-1+Normal $\mathrm{IgG}$ ) and medium containing both rhFGF-1 (10ng/ml) and anti-FGF-1 (rhFGF-1 + anti-FGF-1) served as controls to verify the positive effects of FGF-1 on the proliferation of chondrocytes and the neutralizing activity of the anti-FGF-1 antibody.

\section{Discussion}

Previously we have shown that co-culture of MSCs and PC in pellets augmented cartilage matrix formation. This effect was attributed to a trophic effect of the MSCs on the chondrocytes rather than differentiation of MSCs into chondrocytes. We furthermore showed that in these co-cultures MSC secreted factors potently induced 


\section{Chapter 5}

chondrocyte proliferation [3]. In this study, we have identified FGF-1 as the main MSC secreted growth factor responsible for this effect. We base this conclusion on the following observations; i) $F G F-1$ expression was up-regulated in co-cultures predominantly in the MSCs, ii) FGF-1 is secreted in conditioned medium of co-culture pellets only and in conditioned medium of MSCs cultured in monolayer, and iii) blocking FGF-1 activity either by chemical inhibitors of the FGF-receptor signaling pathway or by an FGF-1 neutralizing antibody potently inhibited chondrocyte proliferation normally induced in co-culture pellets.

Many reports addressing the secretome of MSCs investigated their secretory profile at late stages of adipogenic, osteogenic and chondrogenic differentiation [22-24]. Only a few studies explored the secretome of undifferentiated MSCs [25]. It has been shown in a proteomic study that undifferentiated MSCs secrete a number of matrix components and matrix regulators in 2-D cultures [26], however, expression regulation of these proteins by cellular interactions between MSCs and other cell types are not yet investigated. The current study is one of the first to explore the response and changes in gene expression of MSCs in co-culture with other cell types. Our study provides clear evidence that MSC change the expression and secretion of a number of genes in response to a co-culture with other cell types.

One of the most important observations in this study is that co-culture of MSCs and chondrocytes up-regulated a number of cytokines and growth factors as well as a bunch of matrix remodeling proteins. Among these genes, BMP-2 and FGF-1 stood out, since their functions in chondrocyte proliferation and matrix formation had been previously demonstrated [27-29]. For a long time, BMP-2 has been used as a strong stimulator for osteogenic differentiation of stem cells [30]. In other studies, however, BMP-2 has been applied to promote the chondrogenic differentiation of MSCs as well $[19,21]$. Meanwhile, it is also believed that BMP-2 induced chondrogenic differentiation of MSCs will eventually lead to hypertrophy and endochondralossification [31-32]. Besides BMP-2's contribution to matrix formation, there is a few studying regarding its function in chondrocyte proliferation [29, 33]. However, qPCR data from the xenogenic co-culture model indicated that expression of BMP-2 appeared to be increased predominantly in chondrocytes but not in MSCs. Therefore, BMP-2 is 
very unlike to be a decisive trophic factor produced by MSCs in co-culture pellets, which is the main focus of this study. It is feasible though that up-regulated $B M P-2$ expression in chondrocytes induced by the MSCs in co-culture is at least in part responsible for increased matrix formation in co-culture pellets. This is subject of further studies.

We next paid our attention to the effects of FGF-1 in co-culture. FGF-1 belongs to the fibroblast growth factor super family, which consists of 22 genes in humans and mice [34]. Like other members in the FGF family, FGF-1 is ubiquitous involved in vertebrate embryonic and fetal development, as well as in physiological processes in adult organisms and pathological conditions [35]. It functions by activating a family of tyrosine kinase cell-surface receptors (FGFRs). Unlike FGF-2, FGF-1 is synthesized as one $17.5 \mathrm{kD}$ cytosolic protein [36]. It is expressed in normal physiological conditions but secreted in response to stress, such as injuries and heat shock [37]. There are reports claiming that FGF-1 could be secreted from chondrocytes, chondrogenic precursors and macrophages to stimulate the proliferation of immature chondrocytes, and indirectly accelerate their maturation and deposition of cartilage matrix proteins [20]. Our results also showed that $F G F-1$ was expressed at low levels in chondrocytes and its expression modestly increased in co-culture with MSCs. The dominant source of FGF-1 in co-culture pellets were however the MSCs as shown by immunofluorescent staining in combination with cell tracking and species specific PCR in xenogenic co-cultures.

Traditionally, scientists believed that FGF-2 might play a more important role in the proliferation of chondrocytes than FGF-1 [27, 38-39]. So, many efforts have been made to study the molecular signals involved in FGF-2 induced proliferation of chondrocytes [40-42], with the hope of preventing the formation of fibrous cartilage tissue caused by FGF-2 treatment [43-44]. Our data uncover a positive role of FGF-1 in proliferation of chondrocytes at least in co-culture with MSCs. Our qPCR data obtained from the xenogenic co-culture models as well as results of immunofluorescent staining clearly showed that the expression of FGF-1 in the co-culture pellets is predominantly from MSCs. Combined with our observations that blockade of FGF-1 activity either by inhibitors of FGFR-signaling or by a neutralizing antibody inhibited chondrocyte 


\section{Chapter 5}

proliferation in co-culture pellets and that neutralizing antibodies blocked chondrocyte proliferation induced by MSC conditioned medium, our study identifies FGF-1 as the MSC secreted factor responsible for stimulation of chondrocyte proliferation in cocultures.

Another interesting finding in this paper is the location of the cells expressing $F G F-1$ in co-culture pellets as indicated by immunofluorescent staining. In line with low levels of FGF-1 expression in chondrocytes, some chondrocytes stained positive with FGF-1 antibodies. However, staining in MSCs was more abundant. Interestingly, the most intense FGF-1 staining was found in MSCs which were in close vicinity or even direct contact with chondrocytes. More distant MSCs without physical contacts with chondrocytes either expressed $F G F-1$ at low level or did not express $F G F-1$ at all. This implies that the enhancement of $F G F-1$ expression in co-culture pellets is very likely due to an as yet unknown effect of the chondrocytes on the MSCs. This may either involve a secreted factor or since $F G F-1$ expressing MSCs are invariably in close vicinity of the chondrocytes signaling via direct cell-cell contacts. We are currently exploring both options. Combined with the fact that FGF-1 concentration increased dramatically in the conditioned medium of co-culture pellets when compared with monocultures, we also concluded that not only expression but also secretion of FGF-1 is increased by the cross-talk between MSCs and chondrocytes. From literature it can be deduced that both cells secrete a wide range of growth factors, cytokines and extracellular matrix components into their surrounding environment [26]. Some of these molecules may form a feedback loop that stimulates the expression of $F G F-1$. There are plenty of examples studying the regulatory effects of MSCs on inflammatory responses, in which MSCs create a feedback loop with other cell types to activate or inhibit signaling pathways [45]. In this study we now expand this to mutual cross talk between MSCs and chondrocytes.

In conclusion, our data have identified FGF-1 as an MSC secreted factor which expression is increased in co-cultures with chondrocytes. In turn, FGF-1 potently stimulated chondrocyte proliferation. As far as we know, this study is one of the first dedicated to the analysis of reciprocal changes in gene expression in co-culture pellets of MSCs with other cell types. It provides clear evidence for a mutual relationship 
between MSCs and chondrocytes. It has been proposed that intra-articular injection of MSCs might be a treatment option for osteoarthritis. This is currently under investigation in clinical trials [46]. Our study may provide insight how MSCs exert such beneficial effect on diseased cartilage. In addition, besides to FGF-18 which is explored for clinical application in osteoarthritis, our study identifies FGF-1 as a potential therapeutic agent in osteoarthritis.

\section{Reference:}

1. Schnabel M, Marlovits S, Eckhoff G, et al. Dedifferentiation-associated changes in morphology and gene expression in primary human articular chondrocytes in cell culture. Osteoarthritis Cartilage 2002;10:62-70.

2. Hendriks JAA, Miclea RL, Schotel R, et al. Primary chondrocytes enhance cartilage tissue formation upon co-culture with a range of cell types. Soft Matter 2010;6:5080-5088.

3. Wu L, Leijten JC, Georgi N, et al. Trophic effects of mesenchymal stem cells increase chondrocyte proliferation and matrix formation. Tissue Eng Part A 2011;17:1425-1436.

4. Wu L, Prins HJ, Helder M, et al. Trophic effects of mesenchymal stem cells in chondrocyte cocultures are independent of culture conditions and cell sources. Tissue Eng Part A 2012.

5. Li Y, Chen J, Zhang CL, et al. Gliosis and brain remodeling after treatment of stroke in rats with marrow stromal cells. Glia 2005;49:407-417.

6. Sassoli C, Pini A, Mazzanti B, et al. Mesenchymal stromal cells affect cardiomyocyte growth through juxtacrine Notch-1/Jagged1 signaling and paracrine mechanisms: Clues for cardiac regeneration. J Mol Cell Cardiol 2011.

7. Tang YL, Zhao Q, Qin X, et al. Paracrine action enhances the effects of autologous mesenchymal stem cell transplantation on vascular regeneration in rat model of myocardial infarction. Ann Thorac Surg 2005;80:229-236; discussion 236-227.

8. Walter MN, Wright KT, Fuller HR, et al. Mesenchymal stem cell-conditioned medium accelerates skin wound healing: an in vitro study of fibroblast and keratinocyte scratch assays. Exp Cell Res 2010;316:1271-1281.

9. Yoon BS, Moon JH, Jun EK, et al. Secretory profiles and wound healing effects of human amniotic fluid-derived mesenchymal stem cells. Stem Cells Dev 2010;19:887-902.

10. Caplan AI, Dennis JE. Mesenchymal stem cells as trophic mediators. J Cell Biochem 2006;98:1076-1084.

11. da Silva Meirelles L, Caplan AI, Nardi NB. In search of the in vivo identity of mesenchymal stem cells. Stem Cells 2008;26:2287-2299.

12. Moreira Teixeira LS, Leijten J, Sobral J, et al. High throughput generated micro-aggregates of chondrocytes stimulate cartilage formation in vitro and in vivo. Eur Cell Mater 2012;23:387-399.

13. Hendriks J, Riesle J, Vanblitterswijk CA. Effect of stratified culture compared to confluent culture in monolayer on proliferation and differentiation of human articular chondrocytes. Tissue Eng 2006; 12:2397-2405.

14. Fernandes H, Dechering K, Van Someren E, et al. The role of collagen crosslinking in differentiation of human mesenchymal stem cells and MC3T3-E1 cells. Tissue Eng Part A 2009; 15:3857-3867.

15. Leijten JC, van Blitterwijk CA, Karperien M, et al. GREM1, FRZB and DKK1 are key regulators of human articular cartilage homeostasis. Arthritis Rheum 2012.

16. Livak KJ, Schmittgen TD. Analysis of relative gene expression data using real-time quantitative PCR and the 2(-Delta Delta C(T)) Method. Methods 2001;25:402-408.

17. Szklarczyk D, Franceschini A, Kuhn M, et al. The STRING database in 2011: functional interaction networks of proteins, globally integrated and scored. Nucleic Acids Res 2011;39:D561-568.

18. Abramoff MD, Magelhaes PJ, Ram SJ. Image Processing with ImageJ. Biophotonics 
International 2004;11:36-42.

19. Kurth T, Hedbom E, Shintani N, et al. Chondrogenic potential of human synovial mesenchymal stem cells in alginate. Osteoarthritis Cartilage 2007;15:1178-1189.

20. Bolander ME. Regulation of fracture repair by growth factors. Proc Soc Exp Biol Med 1992;200:165-170.

21. Noth U, Rackwitz L, Heymer A, et al. Chondrogenic differentiation of human mesenchymal stem cells in collagen type I hydrogels. J Biomed Mater Res A 2007;83:626-635.

22. Alvarez-Llamas G, Szalowska E, de Vries MP, et al. Characterization of the human visceral adipose tissue secretome. Mol Cell Proteomics 2007;6:589-600.

23. Ji YH, Ji JL, Sun FY, et al. Quantitative proteomics analysis of chondrogenic differentiation of C3H10T1/2 mesenchymal stem cells by iTRAQ labeling coupled with on-line two-dimensional LC/MS/MS. Mol Cell Proteomics 2010;9:550-564.

24. Chiellini C, Cochet O, Negroni L, et al. Characterization of human mesenchymal stem cell secretome at early steps of adipocyte and osteoblast differentiation. BMC Mol Biol 2008;9:26.

25. Choi YA, Lim J, Kim KM, et al. Secretome analysis of human BMSCs and identification of SMOC1 as an important ECM protein in osteoblast differentiation. J Proteome Res 2010;9:29462956.

26. Polacek M, Bruun JA, Elvenes J, et al. The secretory profiles of cultured human articular chondrocytes and mesenchymal stem cells: implications for autologous cell transplantation strategies. Cell Transplant 2011;20:1381-1393.

27. Ornitz DM. FGF signaling in the developing endochondral skeleton. Cytokine Growth Factor Rev 2005;16:205-213.

28. Shea CM, Edgar CM, Einhorn TA, et al. BMP treatment of C3H10T1/2 mesenchymal stem cells induces both chondrogenesis and osteogenesis. J Cell Biochem 2003;90:1112-1127.

29. Shu B, Zhang M, Xie R, et al. BMP2, but not BMP4, is crucial for chondrocyte proliferation and maturation during endochondral bone development. J Cell Sci 2011;124:3428-3440.

30. Lin Y, Tang W, Wu L, et al. Bone regeneration by BMP-2 enhanced adipose stem cells loading on alginate gel. Histochem Cell Biol 2008;129:203-210.

31. Carlberg AL, Pucci B, Rallapalli R, et al. Efficient chondrogenic differentiation of mesenchymal cells in micromass culture by retroviral gene transfer of BMP-2. Differentiation 2001;67:128-138.

32. Steinert AF, Proffen B, Kunz M, et al. Hypertrophy is induced during the in vitro chondrogenic differentiation of human mesenchymal stem cells by bone morphogenetic protein-2 and bone morphogenetic protein-4 gene transfer. Arthritis Res Ther 2009;11:R148.

33. Li X, Peng J, Wu M, et al. BMP2 promotes chondrocyte proliferation via the Wnt/beta-catenin signaling pathway. Mol Med Report 2011;4:621-626.

34. Itoh N, Ornitz DM. Evolution of the Fgf and Fgfr gene families. Trends Genet 2004;20:563-569.

35. Bottcher RT, Niehrs C. Fibroblast growth factor signaling during early vertebrate development. Endocr Rev 2005;26:63-77.

36. Friesel RE, Maciag T. Molecular mechanisms of angiogenesis: fibroblast growth factor signal transduction. FASEB J 1995;9:919-925.

37. Jackson A, Friedman S, Zhan X, et al. Heat shock induces the release of fibroblast growth factor 1 from NIH 3T3 cells. Proc Natl Acad Sci U S A 1992;89:10691-10695.

38. Ellman MB, An HS, Muddasani P, et al. Biological impact of the fibroblast growth factor family on articular cartilage and intervertebral disc homeostasis. Gene 2008;420:82-89.

39. Goldring MB, Tsuchimochi K, Ijiri K. The control of chondrogenesis. J Cell Biochem 2006;97:33-44.

40. Khan IM, Palmer EA, Archer CW. Fibroblast growth factor-2 induced chondrocyte cluster formation in experimentally wounded articular cartilage is blocked by soluble Jagged-1. Osteoarthritis Cartilage 2010;18:208-219.

41. Khan IM, Evans SL, Young RD, et al. Fibroblast growth factor 2 and transforming growth factor beta1 induce precocious maturation of articular cartilage. Arthritis Rheum 2011;63:3417-3427.

42. Nishida T, Kubota S, Aoyama E, et al. Effect of CCN2 on FGF2-induced proliferation and MMP9 and MMP13 productions by chondrocytes. Endocrinology 2011;152:4232-4241.

43. Yan D, Chen D, Cool SM, et al. Fibroblast growth factor receptor 1 is principally responsible for fibroblast growth factor 2-induced catabolic activities in human articular chondrocytes. Arthritis Res Ther 2011;13:R130.

44. Handorf AM, Li WJ. Fibroblast growth factor-2 primes human mesenchymal stem cells for enhanced chondrogenesis. PLoS One 2011;6:e22887. 
45. Prockop DJ, Oh JY. Mesenchymal stem/stromal cells (MSCs): role as guardians of inflammation. Mol Ther 2012;20:14-20.

46. Kasemkijwattana C, Hongeng S, Kesprayura S, et al. Autologous bone marrow mesenchymal stem cells implantation for cartilage defects: two cases report. J Med Assoc Thai 2011;94:395-400. 


\section{Supplementary materials}

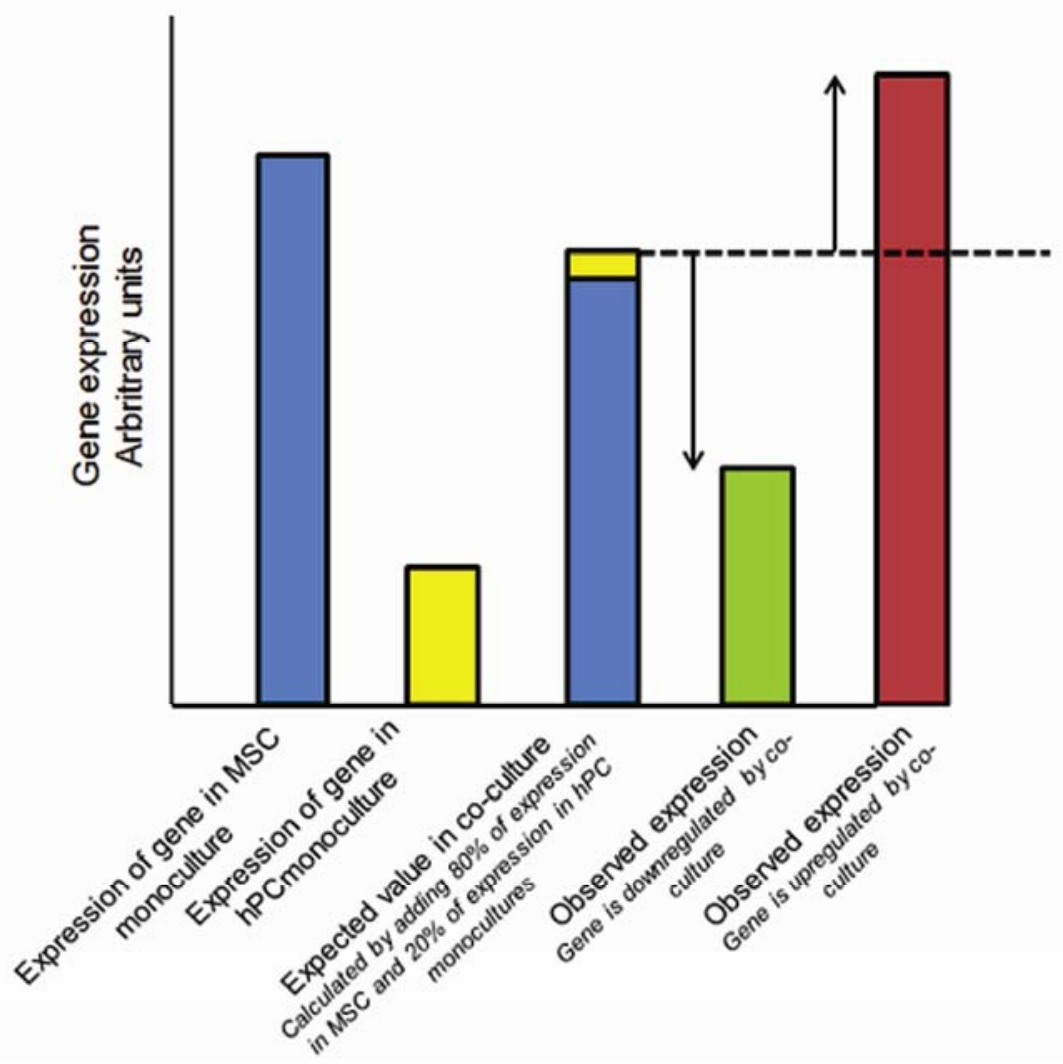

Figure S1. Schematics showing definitions of Observed values and Expected values. Definitions of Expected values (of gene expression in co-culture) and Observed values (of gene expression in co-culture) were made, based on the following assumption: if there is no interaction between hPCs and hMSCs in the coculture pellets, hPCs should contribute $20 \%$ to the expression of the respective gene in co-culture, while hMSCs should contribute $80 \%$, since their seeding ratio is 1:4. Thus, Expected value of one gene in co-culture equals $20 \%$ of its expression in mono-culture of hPCs added to $80 \%$ of its expression in mono-culture of hMSCs, while Observed value reflects the actually expression level of one gene measured by microarray experiment. Genes deviating more than $20 \%$ in expression from the Expected value with a significance level of $p<0.05$ resemble genes of which the expression is regulated by the interaction between hPC and hMSCs and were selected for further analysis. 


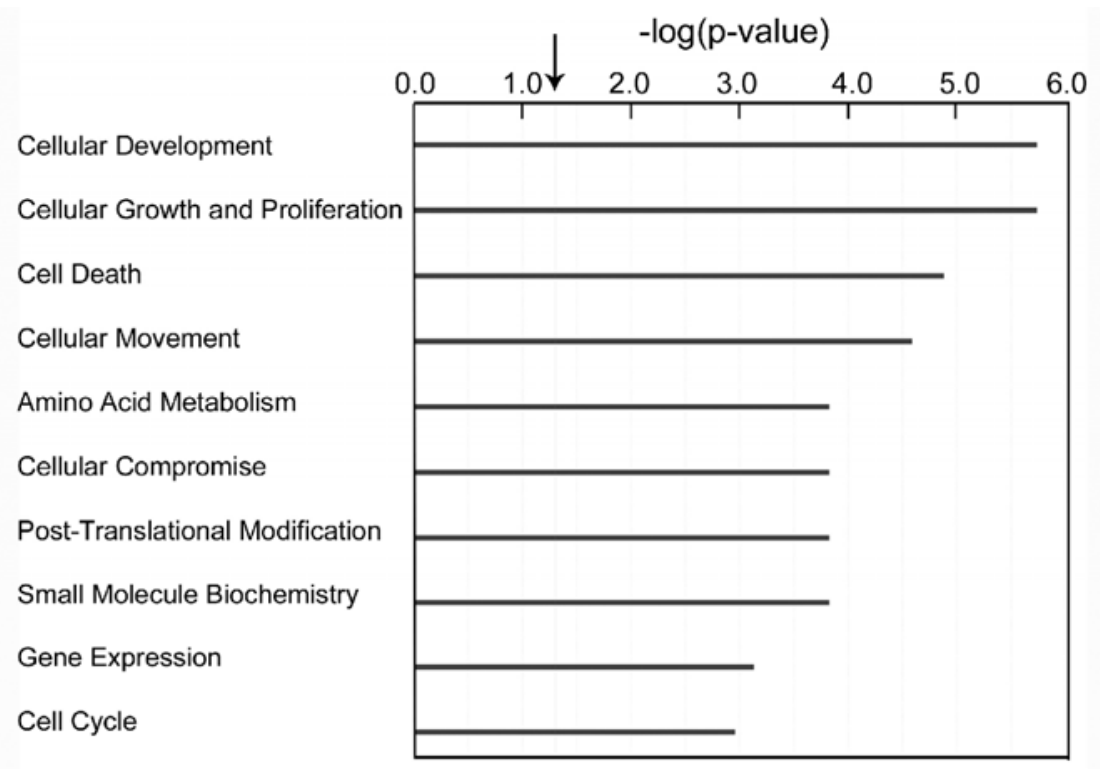

Figure S2. The list of 180 up-regulated genes was imported into ingenuity pathway analysis software to visualize the changes of gene expression in Molecular and cellular function pathway. Top-ten pathways that changed most were listed. Arrow indicates threshold of significance.

$$
-\log (p-v a l u e)
$$

ILK Signaling
Bladder Cancer Signaling
IL-8 Signaling
Atheroscierosis Signaling
Oncostatin M Signaling
Aldosterone Signaling in Epithelial Cells
Cyclins and Cell Cycle Regulation
Potein Ubiquitination Pathway
Regulation of Actin-based Motility by Rho
Endoplasmic Reticulum Stress Pathway

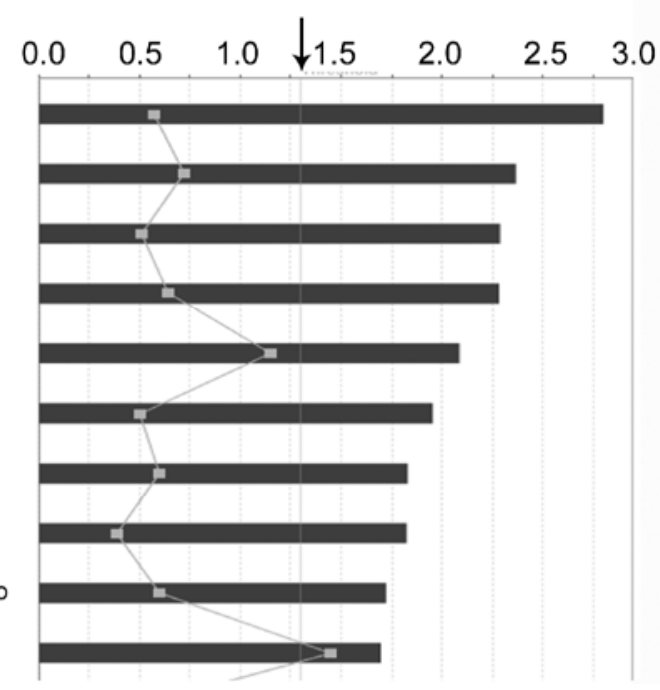

Figure S3. Changes of gene expression in Physiological System Development and Function pathways were analysed in the same way as in Fig S2. Top-ten pathways that changed most were listed. Arrow indicates threshold of significance. 


\section{Chapter 5}

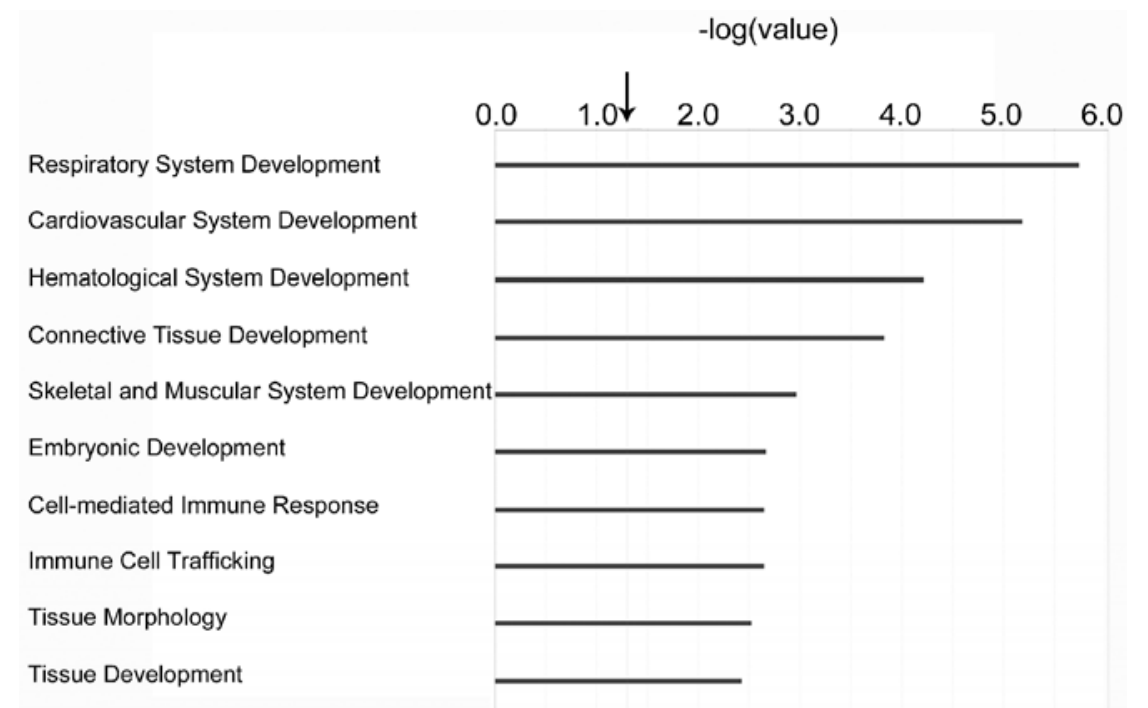

Figure S4. Changes of gene expression in Physiological System Development and Function pathway were analysed in the same way as in Fig S2. Top-ten pathways that changed most were listed. Arrow indicates threshold of significance. 
Supplementary table 1. Forward (F) and Reverse (R) primers used for PCR validation of microarray data.

\begin{tabular}{|c|c|c|c|}
\hline $\begin{array}{l}\text { Gene } \\
\text { Name }\end{array}$ & Primer Sequence & $\begin{array}{l}\text { Product } \\
\text { size }\end{array}$ & Gene Bank No. \\
\hline GAPDH & $\begin{array}{l}\text { F: 5' CGCTCTCTGCTCCTCCTGTT 3' } \\
\text { R: 5'CCATGGTGTCTGAGCGATGT 3', }\end{array}$ & 82 & NM_002046 \\
\hline MMP1 & $\begin{array}{l}\text { F: 5'TTCGGGGAGAAGTGATGTTC3' } \\
\text { R: 5'TTGTGGCCAGAAAACAGAAA3' }\end{array}$ & 101 & NM_002421 \\
\hline $\begin{array}{l}\text { COL15A } \\
1\end{array}$ & $\begin{array}{l}\text { F: 5' CAGTGCTGGTGTCTGCTGAT 3' } \\
\text { R: 5'CCTGGGAAGCAGTCTCTGTC 3' }\end{array}$ & 91 & NM_001855 \\
\hline CHI3L1 & $\begin{array}{l}\text { F: 5' GGGAAGGTCACCATTGACAG 3' } \\
\text { R: 5'GGCTCCATGAAAATCGTAGG 3' }\end{array}$ & 93 & NM_001276 \\
\hline FGF-1 & $\begin{array}{l}\text { F: 5' AGCCGGGCTACTCTGAGAAGAAGA 3' } \\
\text { R: 5'CTGCTGCTTGTGGCGCTTTCA 3' }\end{array}$ & 130 & NM_033136 \\
\hline IGFBP3 & $\begin{array}{l}\text { F: 5' CTCTGCGTCAACGCTAGTGC 3' } \\
\text { R: 5'CGGTCTTCCTCCGACTCAC 3' }\end{array}$ & 95 & NM_000598 \\
\hline BMP-2 & $\begin{array}{l}\text { F: 5' TTGCGCCAGGTCCTTTGACCA 3' } \\
\text { R: 5'CCTGGGGAAGCAGCAACGCT 3' }\end{array}$ & 149 & NM_001200 \\
\hline SIPA1L2 & $\begin{array}{l}\text { F: 5' TGGGGAGCGTTCTCCATCACCA 3', } \\
\text { R: 5' CAGGTGCTGCACTTCTGCTTGGA 3' }\end{array}$ & 130 & NM_020808 \\
\hline CCND1 & $\begin{array}{l}\text { F: 5' CCGCAATGACCCCGCACGAT 3' } \\
\text { R: 5' TGCCACCATGGAGGGCGGAT 3' }\end{array}$ & 155 & NM_053056 \\
\hline CCND2 & $\begin{array}{l}\text { F: 5’ AGCGGGAGAAGCTGTCTCTGATCC 3' } \\
\text { R: 5' TGCTCCCACACTTCCAGTTGCG 3' }\end{array}$ & 119 & NM_001759 \\
\hline CCND3 & $\begin{array}{l}\text { F: 5' ACTCCCCAAAGGCAGGCTCCG 3' } \\
\text { R: 5' GCTGCTCCTCACATACCTCCTCGT 3' }\end{array}$ & 112 & NM_001136017 \\
\hline SPP1 & $\begin{array}{l}\text { F: 5' GCCTTCTCAGCCAAACGCCGA 3' } \\
\text { R: 5' TGGCACAGGTGATGCCTAGGAGG 3' }\end{array}$ & 89 & NM_000582 \\
\hline COL5A3 & $\begin{array}{l}\text { F: 5' GGTCTCTGCCTGCTCCTG 3' } \\
\text { R: 5' CTGGCCTCCCTGCACAC 3' }\end{array}$ & 96 & NM_015719 \\
\hline FGFRL1 & $\begin{array}{l}\text { F: 5' AGGTCCGGACAGGCCGAGAT 3' } \\
\text { R: 5' TGGGACCACCTTGTCCGCCAT 3' }\end{array}$ & 129 & NM_021923 \\
\hline CHI3L2 & $\begin{array}{l}\text { F: 5' TTCTCTTGTCCATTGGAGGG 3' } \\
\text { R: 5' GGAGTTAATGAATTCCAAGCGT 3' }\end{array}$ & 92 & NM_004000 \\
\hline HSPA6 & $\begin{array}{l}\text { F: 5' CGCACCTTCCCGCCCAGTTG 3' } \\
\text { R: 5' ATGCCCCGATCTGCCCGAAC 3' }\end{array}$ & 106 & NM_002155 \\
\hline IFIT1 & $\begin{array}{l}\text { F: 5' GGCAGCCGTTCTGCAGGGTTT 3' } \\
\text { R: 5' ACACCATTGGCTGCTGTTTAGCTCC 3' }\end{array}$ & 131 & NM_00154 \\
\hline IFIT2 & $\begin{array}{l}\text { F: 5' GTTGCCGTAGGCTGCTCTCCAA 3' } \\
\text { R: 5' CCTGAACCGAGCCCTGCCGA 3' }\end{array}$ & 90 & NM_001547 \\
\hline $\begin{array}{l}\text { PLA2G2 } \\
\text { A }\end{array}$ & $\begin{array}{l}\text { F: 5' CAACTTCTGCCCCGGCCGTC 3' } \\
\text { R: 5' CCAGGGAGCATTCACCTGCCC 3' }\end{array}$ & 79 & NM_001161728 \\
\hline
\end{tabular}


Supplementary table 2. Forward (F) and Reverse (R) primers used for species specific qPCR on xenogenic co-culture of bPCs and hMSCs.

\begin{tabular}{|c|c|c|c|}
\hline Gene Name & Primer Sequence & $\begin{array}{l}\begin{array}{l}\text { Product } \\
\text { size }\end{array} \\
\end{array}$ & Gene Bank No. \\
\hline Cross-species & F: 5' AGCTCACTGGCATGGCCTTC 3', & \multirow[t]{2}{*}{116} & NM_002046\& \\
\hline GAPDH & R: 5' CGCCTGCTTCACCACCTTCT 3' & & NM_001034034 \\
\hline Human specific & F: 5' CGCTCTCTGCTCCTCCTGTT 3' & \multirow[t]{2}{*}{82} & \multirow[t]{2}{*}{ NM_002046 } \\
\hline GAPDH & R: 5'CCATGGTGTCTGAGCGATGT 3' & & \\
\hline Bovine specific & F: 5' GCCAT CACTG CCACC CAGAA 3' & \multirow[t]{2}{*}{207} & \multirow[t]{2}{*}{ NM_001034034 } \\
\hline GAPDH & R: 5' GCGGCAGGTCAGATCCACAA 3' & & \\
\hline Human specific & F: 5' TTGCGCCAGGTCCTTTGACCA 3' & \multirow[t]{2}{*}{149} & \multirow[t]{2}{*}{ NM_001200 } \\
\hline BMP2 & R: 5' CCTGGGGAAGCAGCAACGCT 3' & & \\
\hline Bovine specific & F: 5' GAACCCCGCCGCCTCTACCA 3' & \multirow[t]{2}{*}{141} & \multirow[t]{2}{*}{ NM_001099141 } \\
\hline BMP2 & R: 5' CCGCGCCAGGTCCTTCAGC 3' & & \\
\hline Human specific & F: 5' AGCCGGGCTACTCTGAGAAGAAGA 3' & \multirow[t]{2}{*}{130} & \multirow[t]{2}{*}{ NM_033136 } \\
\hline FGF1 & R: 5' CTGCTGCTTGTGGCGCTTTCA 3' & & \\
\hline Bovine specific & F: 5' GCTAGCTCGCTCTGCCGTTCG 3' & \multirow[t]{2}{*}{73} & \multirow[t]{2}{*}{ NM_174055 } \\
\hline FGF1 & R: 5' CCTGGCTCGGTGGGCAATCTG 3' & & \\
\hline Human specific & F: 5’ AGGTCCGGACAGGCCGAGAT 3' & \multirow[t]{2}{*}{129} & \multirow[t]{2}{*}{ NM_021923 } \\
\hline FGFRL & R: 5' TGGGACCACCTTGTCCGCCAT 3' & & \\
\hline Bovine specific & F: 5'ACGTGTGCAAGGCCACCAACG 3' & \multirow[t]{2}{*}{140} & \multirow[t]{2}{*}{ NM_001205996 } \\
\hline FGFRL & R: 5' CTGCTTGCTGGCTGGGTCTTCC 3' & & \\
\hline Human specific & F: 5' GCCTTCTCAGCCAAACGCCGA 3' & \multirow[t]{2}{*}{89} & \multirow[t]{2}{*}{ NM_000582 } \\
\hline SPP1 & R: 5' TGGCACAGGTGATGCCTAGGAGG 3' & & \\
\hline Bovine specific & F: 5' ACTGGACTCTTCTCGCCGCC 3', & \multirow[t]{2}{*}{90} & \multirow[t]{2}{*}{ NM_174187 } \\
\hline SPP1 & R: 5' CGGAGGCAATGCCCAAGAGGC 3' & & \\
\hline Human specific & F: 5' CCGCAATGACCCCGCACGAT 3' & \multirow[t]{2}{*}{155} & \multirow[t]{2}{*}{ NM_053056 } \\
\hline CCND1 & R: 5' TGCCACCATGGAGGGCGGAT 3' & & \\
\hline Bovine specific & F: 5' CTGGGAAGCGCCAACGGCTT 3' & \multirow[t]{2}{*}{112} & \multirow[t]{2}{*}{ NM_001046273 } \\
\hline CCND1 & R: 5' GGGCTTCGATCTGCTCCTGGC 3' & & \\
\hline Human specific & F: 5’ AGCGGGAGAAGCTGTCTCTGATCC3' & \multirow[t]{2}{*}{119} & \multirow[t]{2}{*}{ NM_001759 } \\
\hline CCND2 & R: 5' TGCTCCCACACTTCCAGTTGCG3' & & \\
\hline Bovine specific & F: 5' CTGGCAAAGATCACCAACACCGAT3' & 121 & NM_001076372 \\
\hline CCND2 & R: 5' CCGACTTGGATCCATCGCCCT3' & & \\
\hline Human specific & F: 5' ACTCCCCAAAGGCAGGCTCCG3' & 112 & NM_001136017 \\
\hline CCND3 & R: 5' GCTGCTCCTCACATACCTCCTCGT3' & & \\
\hline Bovine specific & F: 5' ACCACTCTGTCTCTCCCCGCCA 3', & 72 & NM_001034709 \\
\hline CCND3 & R: 5' CCAGGTCCCACTTGAGCTTCCCCAA & & \\
\hline & 3 & & \\
\hline Human specific & F: 5' GCCCAGAGTCCCTTCATAGTC 3' & 130 & NM_020808 \\
\hline SIPA1 & R: 5' GACAGCGAACACAGCTACGA 3' & & \\
\hline Bovine specific & F: 5' GCCCAGAGTCCCTTCATAGTC 3' & 127 & NM_001206220 \\
\hline SIPA1 & R: 5' GACAGCGAACACAGCTACGA 3' & & \\
\hline
\end{tabular}


Supplementary table 3. A full list of up-and down-regulated genes

\begin{tabular}{|c|c|c|}
\hline Symbol & $\begin{array}{l}\text { Ratio } \\
(\mathrm{O} / \mathrm{E})\end{array}$ & Definition \\
\hline \multicolumn{3}{|c|}{ Up-regulated genes } \\
\hline MMP1 & 2.77 & $\begin{array}{l}\text { Homo sapiens matrix metallopeptidase } 1 \text { (interstitial collagenase) (MMP1), } \\
\text { mRNA. }\end{array}$ \\
\hline COL15A1 & 1.88 & Homo sapiens collagen, type XV, alpha 1 (COL15A1), mRNA. \\
\hline CHI3L1 & 1.72 & Homo sapiens chitinase 3-like 1 (cartilage glycoprotein-39) (CHI3L1), mRNA. \\
\hline SIPA1L2 & 1.71 & Homo sapiens signal-induced proliferation-associated 1 like 2 (SIPA1L2), mRNA. \\
\hline CSPG4 & 1.69 & $\begin{array}{l}\text { Homo sapiens chondroitin sulfate proteoglycan } 4 \text { (CSPG4), mRNA. } \\
\text { Homo sapiens fibroblast growth factor } 1 \text { (acidic) (FGF1), transcript variant } 2 \text {, } \\
\text { mRNA.///Homo sapiens fibroblast growth factor } 1 \text { (acidic) (FGF1), transcript } \\
\text { variant } 1 \text {, mRNA. }\end{array}$ \\
\hline C4orf31 & 1.60 & $\begin{array}{l}\text { Homo sapiens chromosome } 4 \text { open reading frame } 31 \text { (C4orf31), mRNA. } \\
\text { Homo sapiens translocated promoter region (to activated MET oncogene) (TPR), }\end{array}$ \\
\hline IGFBP3 & 1.54 & $\begin{array}{l}\text { mRNA. } \\
\text { Homo sapiens insulin-like growth factor binding protein } 3 \text { (IGFBP3), transcript } \\
\text { variant } 1 \text {, mRNA.///Homo sapiens insulin-like growth factor binding protein } 3 \\
\text { (IGFBP3), transcript variant 2, mRNA. }\end{array}$ \\
\hline GPX3 & 1.52 & Homo sapiens glutathione peroxidase 3 (plasma) (GPX3), mRNA. \\
\hline $\begin{array}{l}\text { C18orf51 } \\
\text { LOC10012 }\end{array}$ & 1.48 & $\begin{array}{l}\text { Homo sapiens chromosome } 18 \text { open reading frame } 51 \text { (C18orf51), mRNA. } \\
\text { PREDICTED: Homo sapiens hypothetical protein LOC100129410 }\end{array}$ \\
\hline PPP1R3C & 1.45 & $\begin{array}{l}\text { (LOC100129410), mRNA. } \\
\text { Homo sapiens protein phosphatase 1, regulatory (inhibitor) subunit 3C } \\
\text { (PPP1R3C), mRNA. }\end{array}$ \\
\hline TMED9 & 1.44 & $\begin{array}{l}\text { Homo sapiens transmembrane emp24 protein transport domain containing } 9 \\
\text { (TMED9), mRNA. }\end{array}$ \\
\hline TUBB2B & 1.42 & $\begin{array}{l}\text { Homo sapiens tubulin, beta } 2 B \text { (TUBB2B), mRNA. } \\
\text { Homo sapiens solute carrier family 16, member } 3 \text { (monocarboxylic acid } \\
\text { transporter 4) (SLC16A3), transcript variant 2, mRNA. }\end{array}$ \\
\hline CYP27C1 & 1.41 & $\begin{array}{l}\text { Homo sapiens cytochrome } \mathrm{P} 450 \text {, family } 27 \text {, subfamily } \mathrm{C} \text {, polypeptide } 1 \\
\text { (CYP27C1), mRNA. }\end{array}$ \\
\hline PRICKLE1 & 1.40 & Homo sapiens prickle homolog 1 (Drosophila) (PRICKLE1), mRNA. \\
\hline C13orf33 & 1.39 & Homo sapiens chromosome 13 open reading frame 33 (C13orf33), mRNA. \\
\hline NKD2 & 1.39 & Homo sapiens naked cuticle homolog 2 (Drosophila) (NKD2), mRNA. \\
\hline & 1.38 & Homo sapiens mRNA; cDNA DKFZp586B0220 (from clone DKFZp586B0220) \\
\hline DSG2 & 1.38 & Homo sapiens desmoglein 2 (DSG2), mRNA. \\
\hline $\begin{array}{l}\text { CRYAB } \\
\text { C2CD4B/// }\end{array}$ & 1.37 & $\begin{array}{l}\text { Homo sapiens crystallin, alpha B (CRYAB), mRNA. } \\
\text { PREDICTED: Homo sapiens nuclear localized factor } 2 \text { (NLF2), mRNA.///Homo }\end{array}$ \\
\hline NLF2 & 1.37 & sapiens $\mathrm{C} 2$ calcium-dependent domain containing 4B (C2CD4B), mRNA. \\
\hline PBX2 & 1.36 & $\begin{array}{l}\text { Homo sapiens pre-B-cell leukemia homeobox } 2 \text { (PBX2), mRNA. } \\
\text { Homo sapiens troponin T type } 3 \text { (skeletal, fast) (TNNT3), transcript variant } 1 \text {, }\end{array}$ \\
\hline TNNT3 & 1.36 & mRNA. \\
\hline RRAGD & 1.36 & Homo sapiens Ras-related GTP binding D (RRAGD), mRNA. \\
\hline F3 & 1.35 & Homo sapiens coagulation factor III (thromboplastin, tissue factor) (F3), mRNA. \\
\hline
\end{tabular}




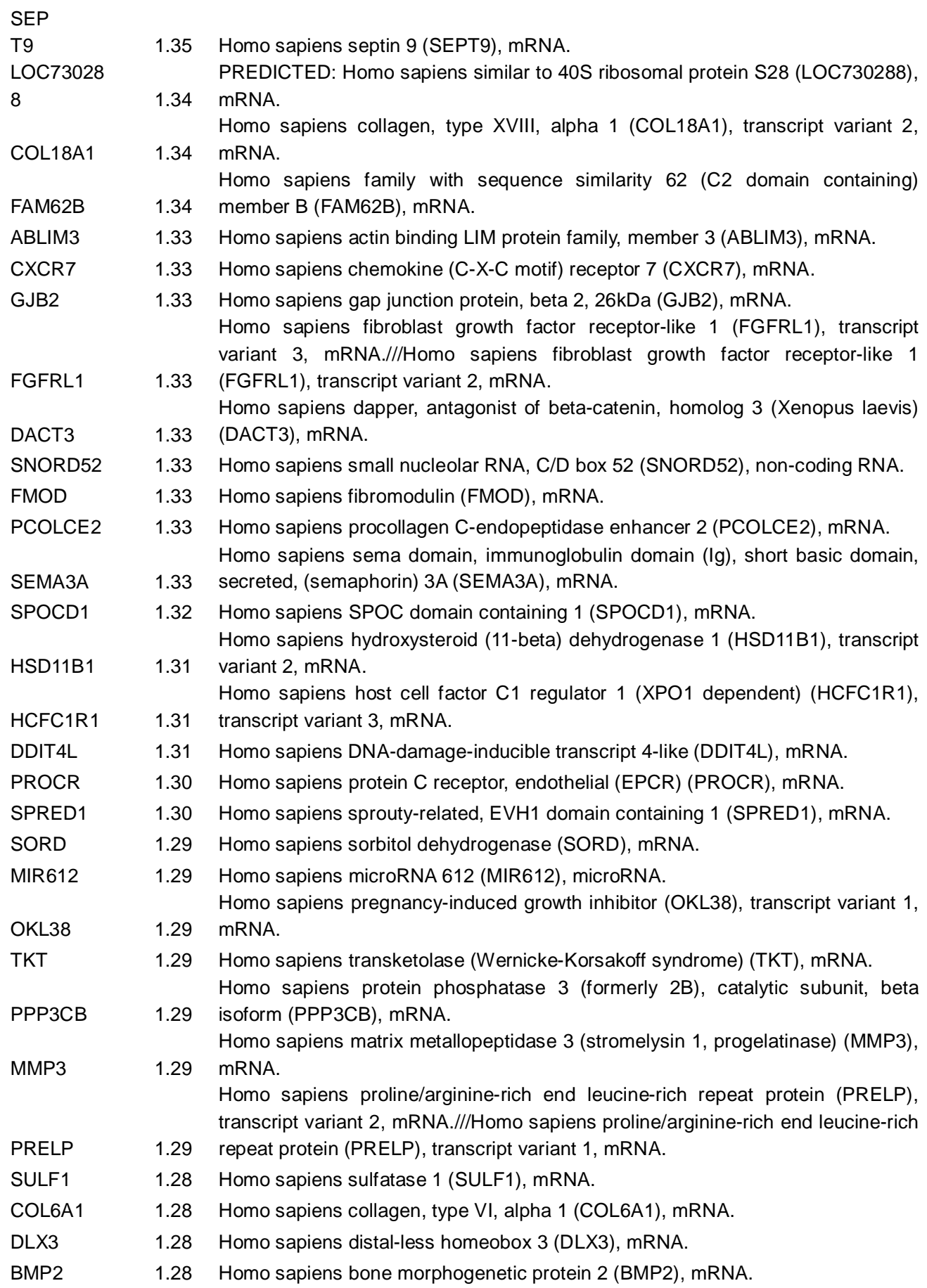




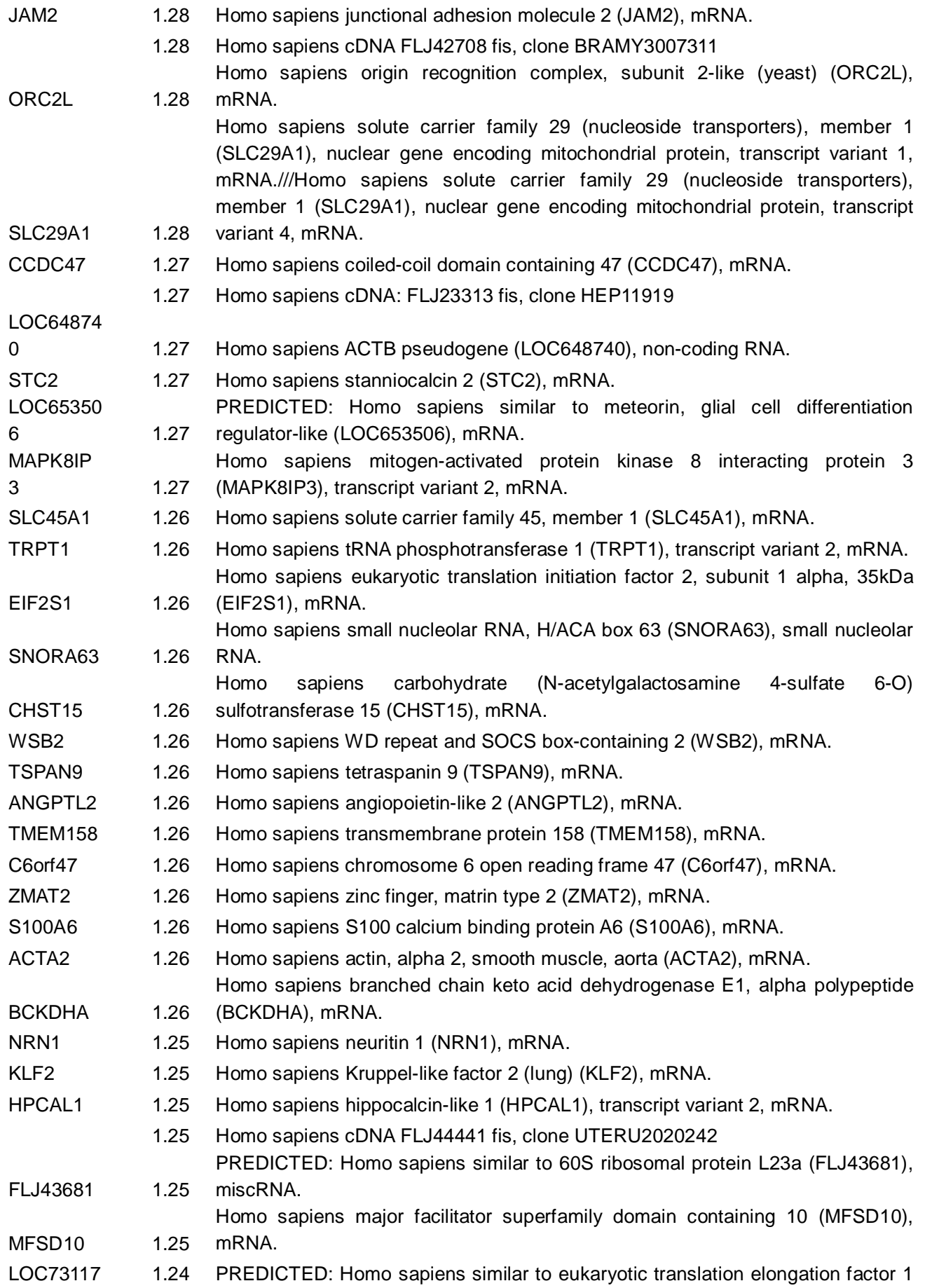


0

BCL2A1

UCHL5IP

PPP2R2C

S100A10

GANAB

CCND1

GLT25D2

AVPI1

WBP2

SRPX2

RPS6KA2

HAPLN1

COMP

ITPRIPL2

PRG4

DCAKD

SPP1

LOC38834

4

FLJ31945

LOC64704

4

ISLR

UBAC1

DUSP23

PHLDB1

MT3

GNB1 alpha 2 (LOC731170), mRNA.

1.24 Homo sapiens BCL2-related protein A1 (BCL2A1), mRNA.

Homo sapiens UCHL5 interacting protein (UCHL5IP), transcript variant 1, 1.24 mRNA.

Homo sapiens protein phosphatase 2 (formerly $2 A$ ), regulatory subunit $B$, gamma is oform (PPP2R2C), transcript variant 2, mRNA.///Homo sapiens protein phosphatase 2 (formerly $2 A$ ), regulatory subunit $B$, gamma isoform (PPP2R2C), 1.24 transcript variant $1, \mathrm{mRNA}$.

Homo sapiens $\mathrm{S} 100$ calcium binding protein A10 (annexin II ligand, calpactin I, light polypeptide (p11)) (S100A10), mRNA.///Homo sapiens $\mathrm{S} 100$ calcium 1.24 binding protein A10 (S100A10), mRNA.

Homo sapiens glucosidase, alpha; neutral AB (GANAB), transcript variant 2, 1.24 mRNA.

1.24 Homo sapiens cyclin D1 (CCND1), mRNA.

1.24 Homo sapiens glycosyltransferase 25 domain containing 2 (GLT25D2), mRNA.

1.24 Homo sapiens arginine vasopressin-induced 1 (AVPI1), mRNA.

1.24 Homo sapiens WW domain binding protein 2 (WBP2), mRNA.

1.24 Homo sapiens sushi-repeat-containing protein, X-linked 2 (SRPX2), mRNA. Homo sapiens ribosomal protein S6 kinase, 90kDa, polypeptide 2 (RPS6KA2), 1.24 transcript variant 2, mRNA.

1.24 Homo sapiens hyaluronan and proteoglycan link protein 1 (HAPLN1), mRNA.

1.24 Homo sapiens cartilage oligomeric matrix protein (COMP), mRNA.

Homo sapiens inositol 1,4,5-triphosphate receptor interacting protein-like 2 1.24 (ITPRIPL2), mRNA.

Homo sapiens proteoglycan 4 (PRG4), transcript variant $C$, mRNA.///Homo

1.23 sapiens proteoglycan 4 (PRG4), transcript variant A, mRNA.

1.23 Homo sapiens dephospho-CoA kinase domain containing (DCAKD), mRNA.

Homo sapiens secreted phosphoprotein 1 (SPP1), transcript variant 1, mRNA.///Homo sapiens secreted phosphoprotein 1 (SPP1), transcript variant 2, 1.23 mRNA.

PREDICTED: Homo sapiens similar to ribosomal protein L13, transcript variant 1

1.23 (LOC388344), mRNA.

PREDICTED: Homo sapiens hypothetical gene supported by AK056507

1.23 (FLJ31945), mRNA.

1.23 PREDICTED: Homo sapiens hypothetical LOC647044 (LOC647044), mRNA. Homo sapiens immunoglobulin superfamily containing leucine-rich repeat (ISLR), transcript variant 2, mRNA.///Homo sapiens immunoglobulin superfamily 1.23 containing leucine-rich repeat (ISLR), transcript variant 1, mRNA.

1.23 Homo sapiens UBA domain containing 1 (UBAC1), mRNA.

1.23 Homo sapiens dual specificity phosphatase 23 (DUSP23), mRNA. Homo sapiens pleckstrin homology-like domain, family B, member 1 (PHLDB1), 1.23 mRNA.

1.23 Homo sapiens metallothionein 3 (MT3), mRNA.

1.23 Homo sapiens guanine nucleotide binding protein (G protein), beta polypeptide 1 
LOC64176

8

COL4A1

ENPEP

LOC10013

1261

PSMD8

LOC72931

7

FAM131A

LOC10012

9608

SNORD33

CCND2

MYOM1

ST13

TRIM8

DLST

DARS

BAG2

FLNB

C18orf32

C7orf59

FBLN7

FAM3A

SLC39A7

DENND5A

VDAC2

BHLHB2

UBE2Q2

CXCL13

CYB5R3

(GNB1), mRNA.

PREDICTED: Homo sapiens similar to ribosomal protein S26, transcript variant 2 (LOC641768), mRNA.

Homo sapiens collagen, type IV, alpha 1 (COL4A1), mRNA.

Homo sapiens glutamyl aminopeptidase (aminopeptidase A) (ENPEP), mRNA.

PREDICTED: Homo sapiens similar to hCG1728885 (LOC100131261), mRNA. Homo sapiens proteasome (prosome, macropain) 26S subunit, non-ATPase, 8 (PSMD8), mRNA.

PREDICTED: Homo sapiens similar to voltage-dependent anion channel 2 (LOC729317), mRNA.

Homo sapiens family with sequence similarity 131, member A (FAM131A), mRNA.

PREDICTED: Homo sapiens hypothetical protein LOC100129608 (LOC100129608), mRNA.

Homo sapiens small nucleolar RNA, C/D box 33 (SNORD33), small nucleolar RNA.

Homo sapiens cyclin D2 (CCND2), mRNA.

Homo sapiens myomesin 1,185kDa (MYOM1), transcript variant 1, mRNA.

Homo sapiens suppression of tumorigenicity 13 (colon carcinoma) (Hsp70 interacting protein) (ST13), mRNA.

Homo sapiens tripartite motif-containing 8 (TRIM8), mRNA.

Homo sapiens dihydrolipoamide S-succinyltransferase (E2 component of 2-oxoglutarate complex) (DLST), mRNA.

Homo sapiens aspartyl-tRNA synthetase (DARS), mRNA.

Homo sapiens BCL2-associated athanogene 2 (BAG2), mRNA.

Homo sapiens filamin B, beta (actin binding protein 278) (FLNB), mRNA.

Homo sapiens chromosome 18 open reading frame 32 (C18orf32), mRNA.

Homo sapiens chromosome 7 open reading frame 59 (C7orf59), mRNA.

Homo sapiens fibulin 7 (FBLN7), mRNA.

Homo sapiens family with sequence similarity 3 , member A (FAM3A), mRNA.

Homo sapiens solute carrier family 39 (zinc transporter), member 7 (SLC39A7), mRNA.///Homo sapiens solute carrier family 39 (zinc transporter), member 7 (SLC39A7), transcript variant 2, mRNA.

Homo sapiens DENN/MADD domain containing 5A (DENND5A), mRNA.

Homo sapiens voltage-dependent anion channel 2 (VDAC2), mRNA.

Homo sapiens basic helix-loop-helix domain containing, class B, 2 (BHLHB2), mRNA.

Homo sapiens ubiquitin-conjugating enzyme E2Q family member 2 (UBE2Q2), mRNA.

Homo sapiens chemokine (C-X-C motif) ligand 13 (B-cell chemoattractant) (CXCL13), mRNA.

Homo sapiens cytochrome b5 reductase 3 (CYB5R3), transcript variant $M$, mRNA.///Homo sapiens cytochrome b5 reductase 3 (CYB5R3), transcript variant $S$, mRNA. 


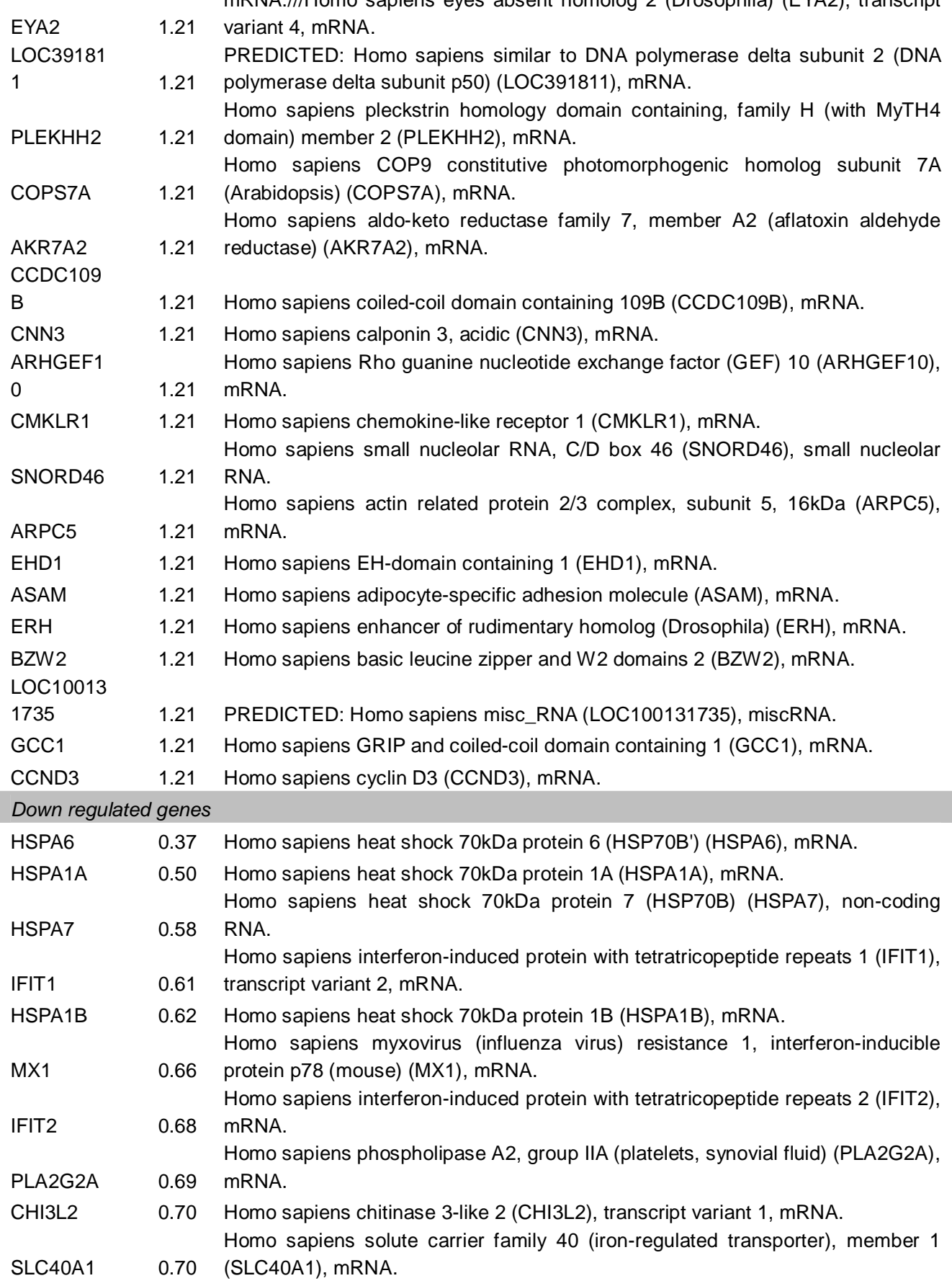

LOC39181

PLEKHH2
COPS7A
AKR7A2
CCDC109
B
ARHGEF1

$$
0
$$

\section{CMKLR1}

SNORD46

ARPC5
EHD1
ASAM
ERH
BZW2
LOC10013
1735
GCC1
CCND3

Down regulated genes

\begin{tabular}{|c|c|c|}
\hline HSPA6 & 0.37 & Homo sapiens heat shock 70kDa protein 6 (HSP70B') (HSPA6), mRNA. \\
\hline HSPA1A & 0.50 & $\begin{array}{l}\text { Homo sapiens heat shock } 70 \mathrm{kDa} \text { protein } 1 \mathrm{~A} \text { (HSPA1A), mRNA. } \\
\text { Homo sapiens heat shock } 70 \mathrm{kDa} \text { protein } 7 \text { (HSP70B) (HSPA7), non-coding }\end{array}$ \\
\hline HSPA7 & 0.58 & RNA. \\
\hline IFIT1 & 0.61 & $\begin{array}{l}\text { Homo sapiens interferon-induced protein with tetratricopeptide repeats } 1 \text { (IFIT1), } \\
\text { transcript variant 2, mRNA. }\end{array}$ \\
\hline HSPA1B & 0.62 & $\begin{array}{l}\text { Homo sapiens heat shock } 70 \mathrm{kDa} \text { protein } 1 \mathrm{~B} \text { (HSPA1B), mRNA. } \\
\text { Homo sapiens myxovirus (influenza virus) resistance } 1 \text {, interferon-inducible }\end{array}$ \\
\hline MX1 & 0.66 & $\begin{array}{l}\text { protein p78 (mouse) (MX1), mRNA. } \\
\text { Homo sapiens interferon-induced protein with tetratricopeptide repeats } 2 \text { (IFIT2), }\end{array}$ \\
\hline IFIT2 & 0.68 & $\begin{array}{l}\text { mRNA. } \\
\text { Homo sapiens phospholipase A2, group IIA (platelets, synovial fluid) (PLA2G2A), }\end{array}$ \\
\hline PLA2G2A & 0.69 & mRNA. \\
\hline CHI3L2 & 0.70 & $\begin{array}{l}\text { Homo sapiens chitinase } 3 \text {-like } 2 \text { (CHI3L2), transcript variant } 1 \text {, mRNA. } \\
\text { Homo sapiens solute carrier family } 40 \text { (iron-regulated transporter), member } 1\end{array}$ \\
\hline SLC40A1 & 70 & (SLC40A1), mF \\
\hline
\end{tabular}

Homo sapiens eyes absent homolog 2 (Drosophila) (EYA2), transcript variant 1, mRNA.///Homo sapiens eyes absent homolog 2 (Drosophila) (EYA2), transcript

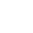


LOC10013

1301

CCDC80

NAG18

SCRG1

PLIN5

THBS1

FOS

OGN

LOC65148

3

LOC39990

0

PILRA

SUGT1P

ZNF483

LOC 10013

4053

USP49

CP

TMEM106

A

PDE4C

LOC64765

0

IFI6

PRRG4

ANGPT2

$\mathrm{RHOU}$

C1RL

LYZ

LOC73020

2

LOC64548

9
0.71 PREDICTED: Homo sapiens misc_RNA (LOC100131301), miscRNA.

Homo sapiens coiled-coil domain containing 80 (CCDC80), transcript variant 1 , 0.71 mRNA.

0.72 Homo sapiens NAG18 protein (NAG18) on chromosome 19.

0.72 Homo sapiens scrapie responsive protein 1 (SCRG1), mRNA.

0.72 Homo sapiens perilipin 5 (PLIN5), mRNA.

0.72 Homo sapiens thrombospondin 1 (THBS1), mRNA.

Homo sapiens v-fos FBJ murine osteosarcoma viral oncogene homolog (FOS),

0.73 mRNA.

0.74 Homo sapiens osteoglycin (OGN), transcript variant 3, mRNA.

0.74 PREDICTED: Homo sapiens hypothetical LOC651483 (LOC651483), mRNA.

0.74 Homo sapiens hypothetical gene supported by AK093779 (LOC399900), mRNA. Homo sapiens paired immunoglobin-like type 2 receptor alpha (PILRA),

0.74 transcript variant 2, mRNA.

PREDICTED: Homo sapiens suppressor of G2 allele of SKP1 pseudogene (S.

0.74 cerevisiae) (SUGT1P), misc RNA.

Homo sapiens zinc finger protein 483 (ZNF483), transcript variant 1 , mRNA.///Homo sapiens zinc finger protein 483 (ZNF483), transcript variant 2,

0.74 mRNA.

PREDICTED: Homo sapiens similar to POLR2J4 protein (LOC100134053),

0.74 mRNA.

0.75 Homo sapiens ubiquitin specific peptidase 49 (USP49), mRNA.

0.75 Homo sapiens ceruloplasmin (ferroxidase) (CP), mRNA.

0.75 Homo sapiens transmembrane protein 106A (TMEM106A), mRNA.

Homo sapiens phosphodiesterase 4C, cAMP-specific (phosphodiesterase E1

0.75 dunce homolog, Drosophila) (PDE4C), mRNA.

PREDICTED: Homo sapiens hypothetical protein LOC647650 (LOC647650), 0.75 mRNA.

Homo sapiens interferon, alpha-inducible protein 6 (IFI6), transcript variant 3 , mRNA.///Homo sapiens interferon, alpha-inducible protein 6 (IFI6), transcript 0.76 variant 2 , mRNA.

xj89b12.x1 Soares_NFL_T_GBC_S1 Homo sapiens cDNA clone

0.76 IMAGE:2664383 3, mRNA sequence

Homo sapiens proline rich Gla (G-carboxyglutamic acid) 4 (transmembrane)

0.76 (PRRG4), mRNA.

0.76 Homo sapiens angiopoietin 2 (ANGPT2), mRNA.

0.76 Homo sapiens ras homolog gene family, member $\mathrm{U}$ (RHOU), mRNA.

0.76 Homo sapiens complement component 1, $r$ subcomponent-like (C1RL), mRNA.

0.76 Homo sapiens lysozyme (renal amyloidosis) (LYZ), mRNA.

PREDICTED: Homo sapiens hypothetical protein LOC730202 (LOC730202),

0.76 miscRNA.

PREDICTED: Homo sapiens hypothetical protein LOC645489 (LOC645489),

0.77 mRNA. 


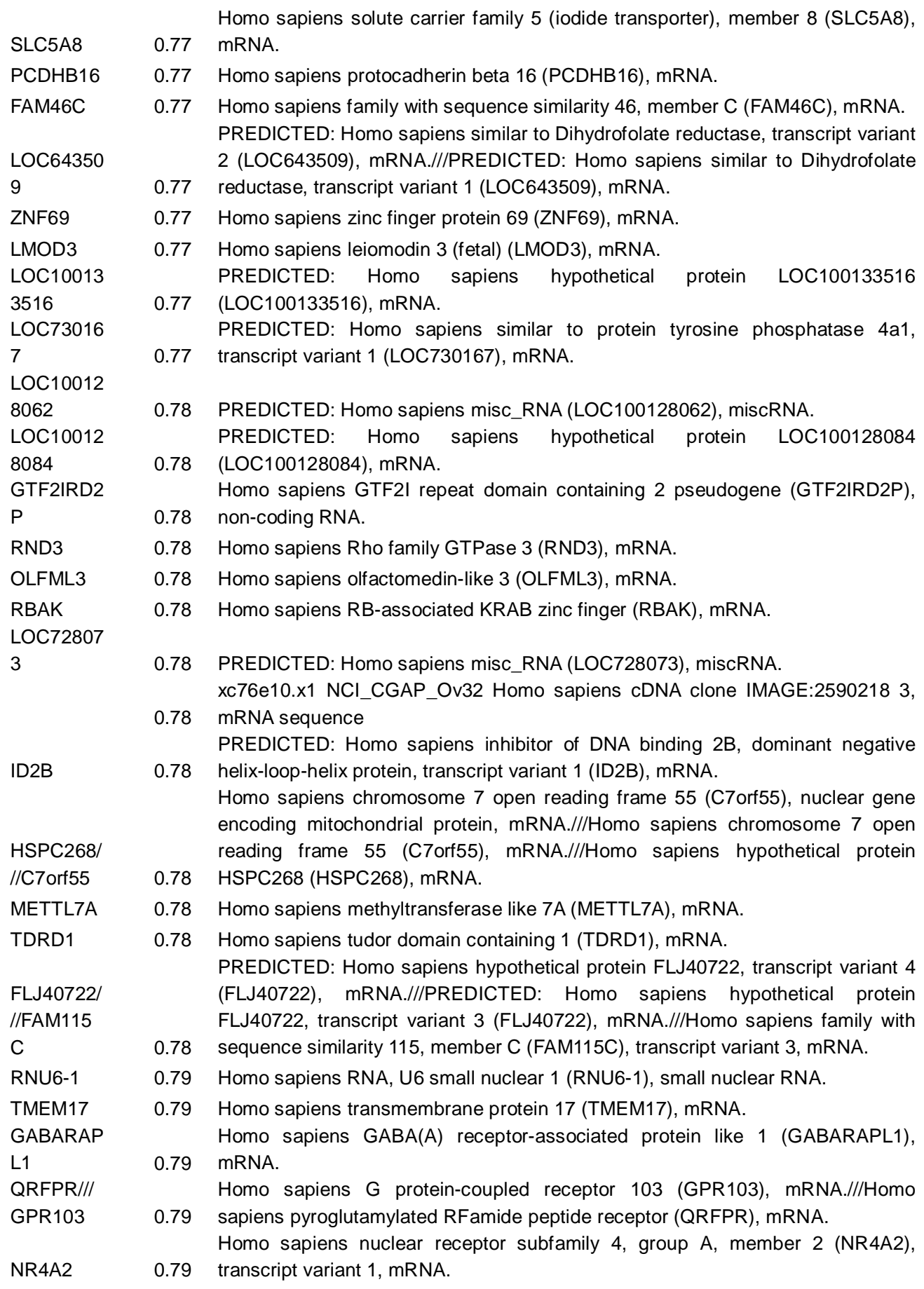




\begin{tabular}{|c|c|c|}
\hline ZNF430 & 0.79 & Homo sapiens zinc finger protein 430 (ZNF430), mRNA. \\
\hline LOC10013 & & hypothetical protein \\
\hline 4364 & 0.79 & (LOC100134364), mRNA. \\
\hline MYO3B & 0.79 & Homo sapiens myosin IIIB (MYO3B), mRNA. \\
\hline ZNF549 & 0.79 & Homo sapiens zinc finger protein 549 (ZNF549), mRNA. \\
\hline NID2 & 0.79 & Homo sapiens nidogen 2 (osteonidogen) (NID2), mRNA. \\
\hline TMEM156 & 0.79 & Homo sapiens transmembrane protein 156 (TMEM156), mRNA. \\
\hline SIK1 & 0.79 & Homo sapiens salt-inducible kinase 1 (SIK1), mRNA. \\
\hline $\begin{array}{l}\text { SPN } \\
\text { LOC73154 }\end{array}$ & 0.79 & Homo sapiens sialophorin (SPN), transcript variant 1, mRNA. \\
\hline 2 & 0.79 & $\begin{array}{l}\text { PREDICTED: Homo sapiens misc_RNA (LOC731542), miscRNA. } \\
\text { Homo sapiens heat shock } 70 \mathrm{kDa} \text { protein } 5 \text { (glucose-regulated protein, } 78 \mathrm{kDa} \text { ) }\end{array}$ \\
\hline HSPA5 & 0.79 & (HSPA5), mRNA. \\
\hline HSPH1 & 0.79 & Homo sapiens heat shock $105 \mathrm{kDa} / 110 \mathrm{kDa}$ protein 1 (HSPH1), mRNA. \\
\hline LRP8 & 0.79 & $\begin{array}{l}\text { Homo sapiens NLR family, pyrin domain containing } 8 \text { (NLRP8), mRNA. } \\
\text { Homo sapiens GTP binding protein overexpressed in skeletal muscle (GEM), } \\
\text { transcript variant } 1 \text {, mRNA.///Homo sapiens GTP binding protein overexpressed } \\
\text { in skeletal muscle (GEM), transcript variant } 2 \text {, mRNA. }\end{array}$ \\
\hline DUSP19 & 0.79 & Homo sapiens dual specificity phosphatase 19 (DUSP19), mRNA. \\
\hline FGD2 & 0.79 & $\begin{array}{l}\text { Homo sapiens FYVE, RhoGEF and PH domain containing } 2 \text { (FGD2), mRNA. } \\
\text { Homo sapiens hypothetical LOC255031 (FLJ35390), transcript variant 1, non- } \\
\text { coding RNA. }\end{array}$ \\
\hline DIA6 & 0.79 & Homo sapiens protein disulfide isomerase family A, member 6 (PDIA6), mRNA. \\
\hline
\end{tabular}


Supplementary table 4. Genes analyzed by ingenuity pathway analysis software

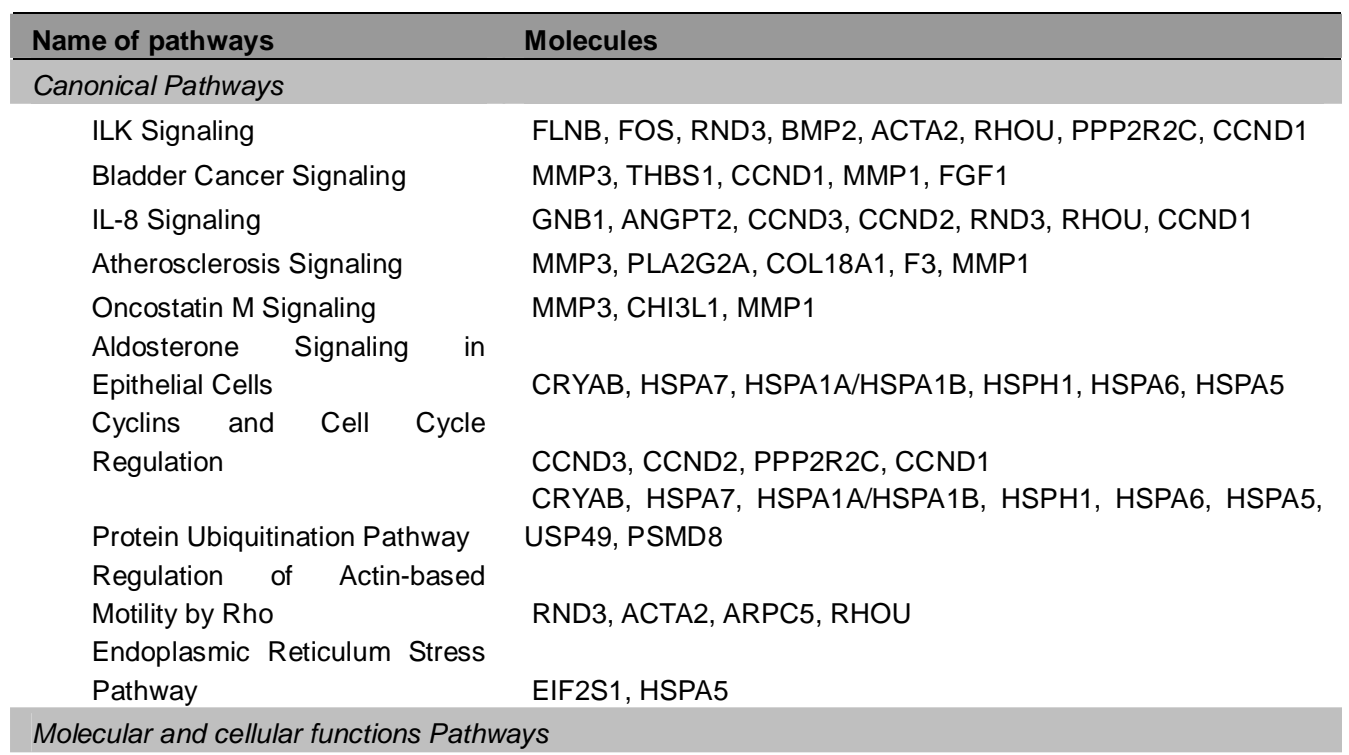

Cellular Development

Cellular Growth and Proliferation

Cell Death

Cellular Movement

Amino Acid Metabolism

Cellular Compromise

Post-Translational Modification
FLNB, SPN, GEM, HSPA1A/HSPA1B, NID2, BMP2, TPR, CCND1, DLST, COL18A1, EYA2, KLF2, COL4A1, SPP1, THBS1, MT3, FGF1, SEMA3A, FOS, CDH2, CCND3, CCND2, NR4A2, IGFBP3, HSD11B1

ENPEP, S100A6, ANGPT2, SPN, HSPA1A/HSPA1B, BMP2, TPR, HSPA5, CCND1, DLST, PRG4 (includes EG:10216), COL18A1, KLF2, COL4A1, SPP1, THBS1, MT3, F3, MT1A, BST2, FGF1, FOS, CDH2, CCND3, CCND2, IGFBP3, PROCR, FGFRL1, BCL2A1

FLNB, ORC2, SULF1, S100A6, ANGPT2, CRYAB, SPN, HSPA1A/HSPA1B, BMP2, HSPA5, COMP, CCND1, GLRX, VDAC2, GNB1, LYZ, CAMK2N1, CSPG4, DLST, IFI6, COL18A1, KLF2, SPP1, PDE4C, THBS1, MT3, MX1, F3, SLC5A8, FGF1, FOSB, FOS, SEMA3A, CDH2, CCND3, NR4A2, RND3, CXCR7, DUSP19, BHLHE40, PROCR, IGFBP3, CHI3L1, BCL2A1, DSG2, FGD2

SULF1, S100A6, ANGPT2, SPN, MMP3, BMP2, TPR, CCND1, PTPRF, TUBB2B, CXCL13, SRPX2, CSPG4, COL18A1, MMP1, S100A10, CMKLR1, SPP1, COL4A1, THBS1, MX1, MAPK8IP3, F3, FGF1, SEMA3A, CDH2, RND3, CXCR7, PROCR, IGFBP3, CHI3L1

ENPEP, CSPG4, BCKDHA, CP, GLRX

CDH2, SPP1, THBS1, CSPG4, IBSP, PLA2G2A, COL18A1, F3 SPP1, SPN, HSPA1A/HSPA1B, THBS1, BMP2, CP, BAG2, GANAB, CCND1, GLRX, CHST15, GNB1, CCND3, CCND2, PDIA6, CSPG4, IGFBP3, SIK1, PRICKLE1 
Small Molecule Biochemistry

Gene Expression

Cell Cycle
ENPEP, SPP1, THBS1, ERH, EEF1A2, MT3, MX1, PLA2G2A, CP, SLC29A1, GLRX, CHST15, FGF1, CSPG4, BCKDHA, HSD11B1

FOS, MMP3, LMO4, HSPA1A/HSPA1B, BMP2, IGFBP3, EYA2, GLRX, CCND1, MMP1

ORC2, SULF1, HSPA1A/HSPA1B, THBS1, BMP2, CCND1, MT1A, CCND3, CCND2, CAMK2N1, BHLHE40, IGFBP3, DARS, RPS6KA2, HAUS7

physiological system development/function pathways

\begin{tabular}{|c|c|}
\hline \multirow{2}{*}{$\begin{array}{l}\text { Respiratory System } \\
\text { Development and Function }\end{array}$} & \\
\hline & CCND2, CCND3, CCND1 \\
\hline Aardiovascular & FLNB, ANGPT2, COL4A1, SPP1, THBS1, BMP2, F3, FGF1, \\
\hline evelopment and Function & CDH2, IGFBP3, PROCR, COL18A1, KLF2 \\
\hline Hematological & SEMA3A, SPP1, CCND2, CCND3, CXCL13, CXCR7, THBS1, \\
\hline nent and Function & CCND1, S100A10 \\
\hline Connective & CCND3, MMP3, BHLHE40, BMP2, TPR, IGFBP3, CCND1, \\
\hline evelopment anc & MMP1, HSD11B1 \\
\hline $\begin{array}{l}\text { keletal and Muscular System } \\
\text { evelopment and Function }\end{array}$ & $\begin{array}{l}\text { FOS, CMKLR1, SPP1, MMP3, THBS1, BHLHE40, BMP2, } \\
\text { PRELP, COMP, DSG2, MMP1, FGF1 }\end{array}$ \\
\hline & ANGPT2, SPP1, COL4A1, BMP2, HSPA5, F3, FGF1, NR4A2, \\
\hline nent & DLST, IGFBP3, RPS6KA2, BCL2A1, EYA2 \\
\hline Cell-mediated & \\
\hline Response & A, SPP1, CXCL1 \\
\hline Immune Cell Trafficking & 7, THBS1, PROCR, S100A10 \\
\hline Tissue Morphology & $\begin{array}{l}\text { ANGPT2, MMP3, IGFBP3, CCND1, MMP1 } \\
\text { ANGPT2, CDH2, COL4A1, SPP1, THBS1, BHLHE40, BMP }\end{array}$ \\
\hline issue Developm & COL18A1, KLF2, CCND1, F3 \\
\hline
\end{tabular}


Chapter 5 


\section{Chapter 6}

\section{Better cartilage formation in chondrocyte co-cultures with the stromal vascular fraction of adipose tissue than with adipose stem cells*}

Ling $\mathrm{Wu}^{1}$, Henk-Jan Prins ${ }^{2,3}$, Jeroen Leijten ${ }^{1}$, Marco N. Helder ${ }^{4}$, Clemens A. van Blitterswijk $^{5}$, Marcel Karperien ${ }^{1}$

${ }^{1}$ Department of Developmental BioEngineering, MIRA-Institute for Biomedical Technology and Technical Medicine, University of Twente, Enschede, 7522 NB, the Netherlands.

${ }^{2}$ Department of Oral Cell Biology, Academic Centre for Dentistry Amsterdam, Amsterdam, the Netherlands.

${ }^{3}$ Department of Oral \& Maxillofacial Surgery, VU Medical Center, Amsterdam, the Netherlands.

${ }^{4}$ Department of Orthopaedics, VU Medical Center, Amsterdam, the Netherlands.

${ }^{5}$ Department of Tissue Regeneration, MIRA-Institute for Biomedical Technology and Technical Medicine, University of Twente, Enschede, 7522NB, the Netherlands.

*Manuscript in preparation 


\begin{abstract}
Partly replacement of chondrocytes by stem cells has been proposed to improve the performance of autologous chondrocytes implantation (ACI). Our previous studies showed that the increased cartilage production in pellet co-cultures of chondrocytes and mesenchymal stem cells (MSCs) is due to a trophic role of the MSC by stimulating chondrocyte proliferation and matrix production rather than MSCs actively undergoing chondrogenic differentiation. The aim of this study is to compare the trophic effects of stromal vascular fraction cells (SVF) and in vitro expanded adipose stem cells (ASC). SVF and culture expanded ASCs ( $n=9)$ were co-cultured with chondrocytes in pellets. By GAG and DNA assay, we showed that co-culture pellets of SVF and chondrocytes have more GAG deposition than that of ASC and chondrocytes. Results of Short Tandem Repeats analysis indicated that increase of the chondrocytes proportion in the co-culture pellets is more pronounced in the SVF coculture group than the ASC co-culture group. Using flow cytometry and microarray, we demonstrated that SVF and ASC have different characteristics in cell surface markers and gene expression profile. SVF is more heterogeneous than ASC, while ASC is more differentiated into mesenchymal lineage than SVF. By subcutaneous implantation into nude mice, we showed that constructs of SVF and choncrocytes are better in depositing cartilage matrix than mixture of ASC and chodnrocytes. Taken together, SVF is better than ASCs in terms of forming cartilage matrix in a co-culture or co-implantation model. Without in vitro expansion, SVF is demonstrated as better cell source for cartilage repair.
\end{abstract}




\section{Introduction}

As self-repair capacity of cartilage tissue is very limited, full-thickness articular cartilage defects usually lead to the development of osteoarthritis, resulting in serious pain and movement limitations [1]. Current treatments for cartilage defects in young patients include microfracturing [2], mosaicplasty and autologous chondrocyte implantation (ACI) [3]. However, each of these treatments has limitations. Fibrin cartilage is usually formed after microfracturing which has inferior mechanical properties compared to native cartilage tissue [4]. Mosaicplasty often causes donor site morbidity and postoperative pain [5]. The drawbacks of ACI are the necessity of two surgical interventions in a time span of several weeks which is required for the obligatory cell expansion in vitro, dedifferentiation of chondrocytes during this in vitro expansion and unpredictable long-term outcome [4, 6]. Therefore, clinicians never stopped looking for new therapies that regenerate cartilage defects without the upmentioned issues. With the progress of stem cell biology in recent years, it has been proposed to replace part of chondrocytes in ACI by other cell sources to avoid the presently obligatory costly and time consuming cell expansion phase. Such approach would enable one step surgery. Previous studies have mainly focused on bone marrow derived mesenchymal stem cells (BM-MSCs) [7-9]. However, partial replacement of chondrocytes with BM-MSCs is far from optimal because of pain and donor site morbidity during isolation and the relative low amount of BM-MSCs in bone marrow aspirates. Other sources of MSCs are therefore becoming more and more attractive.

Adipose tissue-derived stromal cells or adipose stem cells (ASCs) are attractive sources for cartilage tissue engineering, because they are abundant in adipose tissue which is more easily accessible than bone marrow [10]. These cells can easily isolated by liposuction with relatively low morbidity and pain. Most importantly, ASCs are, like BM-MSCs, able to differentiate into various cell types of the mesodermal lineage, including adipocytes, osteoblasts, chondrocytes and myocytes under specific culture conditions in vitro [11]. Isolation of ASCs usually involves several steps: tissues are first minced, digested with collagenase, and fractionated by differential centrifugation. The resulting pelleted stromal vascular fraction (SVF) is resuspended and then placed 
in culture flasks [12] to isolate the plastic adherent ASC subpopulation. Most researchers have focused on the expanded plastic adherent ASC subpopulation, while limited work has been done using the minimally processed SVF cell population. Recent reports have indicated that SVF cells and ASCs exhibit different features and properties [13-14]. From the perspective of clinical practice, SVF cells have great advantages over ASCs, since it's possible to harvest them during the operative procedure itself by processing in the operation theatre and put them back into the patient without laboratory expansion which is required for the isolation of ASCs. Because adipose tissue is an abundant source of stem cells, cell numbers required for re-implantation can easily be obtained.

In our previous report [15], pellet co-culture of chondrocytes and bone marrow derived human mesenchymal stem cells (hMSCs) was shown to benefit cartilage matrix formation. In these pellet co-cultures, we showed that cartilage matrix genes were mainly expressed by chondrocytes. Furthermore, we showed that the ratio of MSCs decreased dramatically due to massive cell death of MSCs by apoptosis and by stimulation of chondrocyte proliferation in co-culture with MSCs. The stimulation of proliferation was at least partly mimicked by culturing chondrocytes in MSC conditioned medium. These findings were confirmed by an independent study performed by Acharya et al.[16]. This and our study demonstrated a new mechanism of cellular interaction in co-culture pellets of MSCs and chondrocytes. Both studies showed that the beneficial effects on cartilage matrix formation in co-culture pellets of MSCs and chondrocytes were due to trophic effects of MSCs stimulating chondrocyte proliferation and cartilage matrix deposition rather than MSCs actively undergoing chondrogenic differentiation. Our follow-up study then showed that these trophic effects are independent of culture conditions and was found in co-cultures of chondrocytes with various sources of MSCs [17]. The term trophic effects initially refers to bioactive molecules produced by nerve terminals, which are not neurotransmitters[18]. In relation to MSCs, the term trophic was first used to describe the process in which MSCs secrete factors that stimulate nearby cells to release functionally bioactive molecules[19]. Later, the term also relates to the effect of the factors produced by MSC on viability, proliferation, and matrix production of the neighboring cells. This concept has resulted 
in a paradigm shift in the way MSCs are involved in tissue repair. While traditionally it was believed that MSCs mainly repair damaged tissue by differentiating into specific cell types and replacing lost cells[20], nowadays the trophic role of the MSC in tissue repair is considered more important[21]. Besides in cartilage regeneration, these effects were also reported in promoting gain of coordinated functions in stroked brain of rats [22], stimulating cardiomyocyte proliferation [23], and vascular regeneration [24].

Our previous studies demonstrated the trophic effects of expanded ASCs [17]. However, use of ASC in partial replacement of chondrocytes in ACI still needs isolation of adipose tissue by plastic adherence followed by cell expansion prior to joint surgery. SVF have the advantage that no expansion is required so that it could be easily incorporated into single step surgery. In this study we have compared SVF and ASC as alternative cell source for replacing part of the chondrocytes in a pellet co-culture system and have evaluated cartilage formation in vivo using an ectopic cartilage formation nude mouse model. Our data suggested that SVF is a better source than ASC for a co-implantation strategy with primary chondrocytes in cartilage repair. This study demonstrates the potential of developing a one-step surgerical procedure for the regeneration of focal cartilage defects using adipose tissue.

\section{Materials and Methods}

\section{Cell culture and expansion}

The use of all human materials in this study has been approved by a local Medical Ethical Committee. Human primary chondrocytes (hPCs) were obtained from macroscopically healthy cartilage obtained from knee or hip biopsies of patients with end stage osteoarthritis undergoing total knee or hip replacement. Cartilage biopsies were digested for $20-22 \mathrm{~h}$ in collagenase type II (0.15\% Worthington, NJ, US) dissolved in chondrocytes proliferation medium. The components of chondrocytes proliferation medium are DMEM supplemented with $10 \%$ FBS, $1 \times$ non-essential amino acids, 0.2mM Ascorbic acid 2-phosphate (AsAP), $0.4 \mathrm{mM}$ proline, 100U/ml penicillin and $100 \mu \mathrm{g} / \mathrm{ml}$ streptomycin. More details about chondrocyte isolation are described elsewhere [25]. Stromal Vascular Fraction (SVF) of human adipose tissue was isolated according to procedures in previous publications [26-27]. SVF were then seeded in 
culture flasks with MSC proliferation medium ( $\alpha$-MEM supplemented with $10 \%$ fetal bovine serum, $1 \%$ L-glutamin, $0.2 \mathrm{mM}$ ascorbic acid, $100 \mathrm{U} / \mathrm{ml}$ penicillin, $10 \mu \mathrm{g} / \mathrm{ml}$ streptomycin and $1 \mathrm{ng} / \mathrm{ml} \mathrm{bFGF}$ ). Media were refreshed every 2 days to get rid of nonattached cells. When confluent, cells were trypsinized and passaged. The cells grown on culture plastic were cultured to passage 2 before experimentation. Expanded cells were called adipose stem cells (ASC) in this paper. All reagents used for cell culture were purchased from Gibco, Invitrogen (Paisley, UK), unless otherwise stated. Common chemicals were purchased from Sigma-Aldrich.

\section{Pellet culture and chondrogenic differentiation}

For mono-cultures, 200,000 cells of hPCs, SVF or ASC were seeded in one well of a round bottom 96 wells plate (non-tissue culture treated). For co-cultures, 200,000 cells were seeded in a ratio of $80 \%$ SVF or ASC and $20 \%$ hPC. Cells were initially seeded in chondrocyte proliferation medium and centrifuged for $5 \mathrm{~min}$ at $500 \times \mathrm{g}$. Medium was changed to chondrogenic differentiation medium (DMEM supplemented with $40 \mu \mathrm{g} / \mathrm{mL}$ of proline, $50 \mathrm{ug} / \mathrm{mL}$ ITS-premix, $50 \mathrm{ug} / \mathrm{mL}$ of AsAP, $100 \mathrm{ug} / \mathrm{mL}$ of Sodium Pyruvate, $10 \mathrm{ng} / \mathrm{mL}$ of TGF $\beta 3,10^{-7} \mathrm{M}$ of dexamethasone, 500 $\mathrm{ng} / \mathrm{mL}$ of BMP6, 100U penicillin/ml and $100 \mu \mathrm{g} / \mathrm{ml}$ streptomycin) one day after seeding when stable pellets were formed. Cell pellets were cultured for 4 weeks before analysis.

\section{Monoclonal antibody labeling and flow cytometry}

The SVF (after one freeze-thaw cycle) and culture-expanded ASCs were analyzed for their cell surface marker expression using monoclonal antibodies against human CD29, CD31, CD34 (clone 8G12), CD45, CD54, CD73, CD90, CD105, CD106, CD117, HLA-ABC, HLA-DR, and Lin1 (all from Becton Dickinson Biosciences, San José, CA, USA), CD146 (Chemicon, Temecula, CA, USA), CD166 (AbD SeroTec/MorphoSys, Oxford, UK), and CD271 (Miltenyi Biotec BV, Bergisch Gladbach, Germany). All monoclonal antibodies used were of the IgG1 type and either fluorescein isothiocyanate or phycoerythrin conjugated. Cells were washed with PBS and stained with specific antibodies for 30 minutes at $4^{\circ} \mathrm{C}$. Nonspecific fluorescence was determined by incubating cells with conjugated mAb anti-human IgG1 (BD). Samples were washed twice and analyzed in a FACS Caliber flow cytometer (BD) with the Cellquest Pro software (BD). 


\section{Histology}

Cell pellets were fixed with $10 \%$ formalin for $15 \mathrm{~min}$, dehydrated with ethanol and embedded in paraffin using routine procedures. A microtome (Shandon, France) was used to cut $5 \mu \mathrm{m}$ thick sections. Slides were then deparaffinized and stained for sulfated glycosaminoglycans (GAG) with Alcian blue or Toluidine blue. Nuclei were counterstained with nuclear fast red.

\section{Quantitative GAG and DNA assays}

Cell pellets $(n=6)$ were washed with phosphate-buffered saline (PBS) and stored at $-80^{\circ} \mathrm{C}$ for $16-20$ hours. Subsequently, they were digested in digestion buffer (1 $\mathrm{mg} / \mathrm{ml}$ proteinase $\mathrm{K}$ in Tris/EDTA buffer ( $\mathrm{pH}$ 7.6) containing $18.5 \mu \mathrm{g} / \mathrm{ml}$ iodoacetamide and $1 \mu \mathrm{g} / \mathrm{ml}$ pepstatin A) for more than $16 \mathrm{~h}$ at $56{ }^{\circ} \mathrm{C}$. GAG content was spectrophotometrically determined with 1,9-dimethylmethylene blue chloride (DMMB) staining in PBE buffer (14.2g/L Na $\mathrm{NPO}_{4}$ and 3.72g/L Na $\mathrm{H}_{2}$ EDTA, pH 6.5) using an ELISA reader (TECAN, Grodig, Austria) at an absorbance of $520 \mathrm{~nm}$ with chondroitin sulfate as a standard. Cell numbers were determined by quantification of total DNA using a CyQuant DNA Kit (Molecular Probes, Eugene, OR).

\section{Immunocytochemstry and immunofluorescent staining}

For immunocytochemistry, sections were deparaffinized, incubated with $3 \%$ hydrogen peroxide and blocked in $1 \%$ bovine serum albumin and $1.5 \%$ normal goat serum. Slides were subsequently incubated overnight at $4{ }^{\circ} \mathrm{C}$ with mouse monoclonal antibodies against COL II (Novagen, Darmstadt, Germany). Sequentially, primary antibodies were visualized by EnVision Detection Systems (Dako, Heverlee, Belgium). Counterstaining was performed with hematoxylin. For immunofluorescent staining, cryosections were incubated with the same antibody against COL II, followed by incubation with secondary antibodies labeled with Alexa 564 (Invitrogen). Counterstaining was performed with DAPI.

\section{RNA isolation and quantitative PCR}

RNA samples of cell pellets were isolated with the NucleoSpin RNA II Kit (Macherey-Nagel, Düren, Germany). Total RNA was reverse-transcribed into cDNA using the iScript cDNA Synthesis kit (Bio-Rad, Hercules, CA). Quantitative PCR (qPCR) was performed on cDNA samples using the iQ SYBR Green Supermix (Bio- 
Rad, Hercules, CA). PCR Reactions were carried out on MyiQ2 Two-Color Real-Time PCR Detection System (Bio-Rad, Hercules, CA) under the following conditions: cDNA was denatured for $5 \mathrm{~min}$ at $95{ }^{\circ} \mathrm{C}$, followed by 45 cycles, consisting $15 \mathrm{~s}$ at $95^{\circ} \mathrm{C}, 15 \mathrm{~s}$ $60^{\circ} \mathrm{C}$ and $30 \mathrm{~s}$ at $72^{\circ} \mathrm{C}$. For each reaction a melting curve was generated to test primer dimer formation and non-specific priming. The sequences of primers for real-time PCR were listed in supplementary table T1. Calculation of Relative Expression was performed with Bio-Rad iQ5 optical system software (version 2.0) using the double delta $\mathrm{Ct}$ method[28]. GAPDH primers were used for normalization.

\section{Microarray processing and statistical analysis}

For microarray study, SVF and ASC from the same donor were used for RNA isolation, 3 donors were used in total. RNA was also isolated from the cells immediately thawed from liquid nitrogen. RNA extraction was performed as described in the previous section. NuGEN Ovation PicoSL WTA System kit followed by Encore BiotinIL module was utilized for synthesizing biotinylated sscDNA starting from $50 \mathrm{ng}$ of total RNA. Obtained Samples (750 ng) were then hybridized onto Illumina HumanHT-12 v4 Expression BeadChips. Chips were scanned by the Illumina iScan array scanner. Illumina's Genomestudio v. 2010.3 software was applied to analyze gene expression profiling using the default settings advised by Illumina. Raw data of fluorescence intensity were normalized by quantile normalization. Differential gene expression was analyzed by the commercial software package Genespring, version 11.5.1. (Agilent Technologies). Genes with at least 2-folds difference and being significantly differentially expressed according to a one way ANOVA with a Benjamini-Hochberg FDR correction and Tukey HSD post hoc test using a cut-off rate of $\mathrm{P} \leq 0.05$ were selected. Changes of gene expression in annotated canonical pathways and bio-functions were analyzed by using ingenuity pathway analysis software (Ingenuity Systems). Search Tool for the Retrieval of Interacting Genes/Proteins (STRING) was used to predicted possible protein-protein interaction network [29]. Clusters were formed using a k-means clustering algorithm.

\section{Cell tracking with organic fluorescent dyes}

The organic fluorescent dye CM-DiI (Molecular Probes, Eugene, OR) was used for cell tracking in co-cultures. Cells were labeled according to the manufacturer's 
protocol. Briefly, cells were trypsinized and resuspended in PBS at a concentration of $2 \times 10^{6}$ cells $/ \mathrm{ml}$. The cells were incubated with $4 \mu \mathrm{M}$ of $\mathrm{CM}$-DiI at $37^{\circ} \mathrm{C}$ for 5 minutes followed by incubation at $4^{\circ} \mathrm{C}$ for 15 minutes. Cells were washed with PBS and applied in co-culture experiments.

\section{5-ethynyl-2'-deoxyuridine labeling and staining}

Cell proliferation in pellets was examined with the Click-iT® EdU Imaging Kit (Molecular Probes, Eugene, OR). Cell pellets were cultured in chondrogenic differentiation medium containing $10 \mu \mathrm{M}$ EdU (5-ethynyl-2'-deoxyuridine) for 24 hours before harvesting. At day 2, cell pellets were washed with PBS and fixed with 10\% formalin for $15 \mathrm{~min}$. Sections of $10 \mu \mathrm{m}$ were cut with a cryotome (Shandon, France). Sections were permeabilized with $0.5 \%$ of Triton X 100 for 20 min and stained for EdU with Alexa 488 cocktail. Nuclei were counterstained with Hoechst 33342 (provided in the same kit).

\section{Image acquisition and analysis}

Histological images were made with a Nikon E300 microscope (Japan). Fluorescent images were taken with a BD pathway 435 confocal microscope (BD Biosciences, Rockville, MD). Details of images quantification were published elsewhere[15]. Values represent the mean \pm standard deviation of at least 3 biological replicates.

\section{Short Tandem Repeats (STR) analysis}

Genomic DNA samples were extracted from pellets with the QIAamp DNA Mini Kit (Qiagen). The sixteen loci of the kit PowerPlex 16 System (Promega) were amplified, typed, sequenced and analyzed by ServiceXS B.V. (Leiden, the Netherlands). Specific alleles for the donor of SVF or ASCs and the donor of hPCs were identified. These alleles were used to define the origin of cells in allogeneic co-culture of hMSCs and hPCs after 4 weeks. The amount of DNA present for each donor was calculated from the areas of the electrophorogram for each locus of SVF or ASC and hPC specific alleles and the ratio of hMSCs and hPCs was determined.

\section{Implantation of cells incorporated in alginate gel into nude mice}

Pools of SVF or ASC were made by mixing cells from 3 donors. hPCs from another 3 donors were mixed to make a pool of hPCs. Then, pools of SVF or ASC were 
mixed with the pool of hPC in a ratio of $4: 1$, and then resuspended in $2 \%$ alginate in PBS at a density of $1 \times 10^{7}$ cells $/ \mathrm{ml}$. Co-implantation constructs were made by transferring $70 \mu \mathrm{l}$ of alginate cell suspension to $100 \mathrm{mM} \mathrm{CaCl}$, solution and gelifying for 5 minutes at $37{ }^{\circ} \mathrm{C}$. Constructs were washed with PBS and MSC proliferation medium. Constructs with only chondrocytes of the same cell density and the volume of alginate cell suspension were served as positive control, while blank constructs without any cells were negative controls. For each condition $(\mathrm{SVF}+\mathrm{CH}, \mathrm{ASC}+\mathrm{CH}, \mathrm{CH}$ and blank), ten constructs were made one day before the surgery and cultured in chondrocyte proliferation medium. Before the implantation, ten 6-week-old male BALB/C nude mice (Experimental Animal Center at UMC-Utrecht) were anesthetized. Then, four subcutaneous pockets were made on the back of a mouse. One construct was put in one pocket.. The locations of the constructs were randomized and recorded. At 8 weeks post-implantation, mice were sacrificed and implanted constructs were then carefully separated from surrounding fibrous capsule, washed in PBS for histological analysis and quantitative GAG analysis.

\section{Statistical analysis}

GAG and DNA quantifications were examined for statistical significance with one-way analysis of variance (ANOVA) followed by Tukey HSD (Honestly Significant Difference) Test. Statistical analysis of EdU positive cells was made by using the Student's $t$ test. $P$ values of $<0.05$ were considered as statistically significant.

\section{Results}

\section{Comparisons of surface marker profiles and chondrogenic potential of SVF and} ASC

We first performed FACs to analysis the profiles of CD markers of the SVF and ASCs. Representative flow histograms are shown in Figure 1A. The mean percentages of positive cells are displayed in Figure 1B. The SVF fraction contained a subset of cells that were positive for the endothelial cell-associated marker CD31, or the hematopoietic lineage associated markers CD34 and CD45. As expected, ASCs did not express these markers. A fraction of the initial SVF cell population expressed stromal cell-associated markers. Only $10 \%$ of the SVFs expressed CD105, whereas $86 \%$ of the 
A
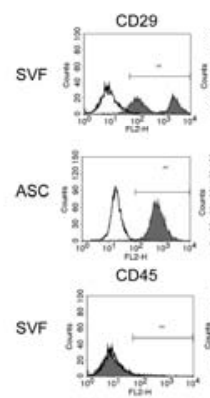

ASC
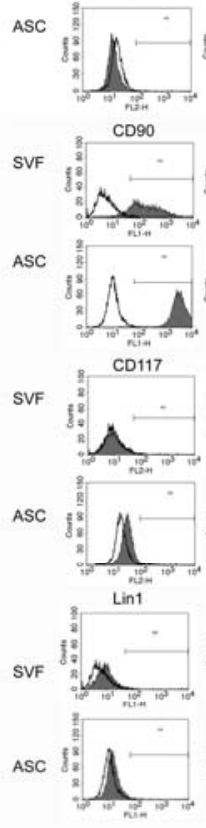
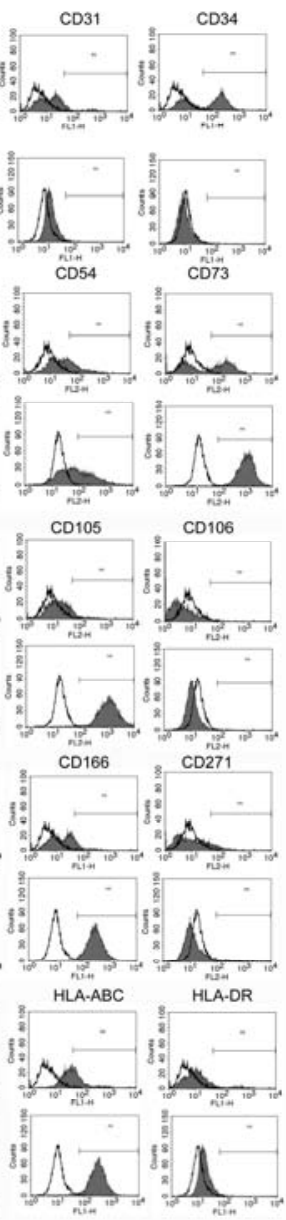
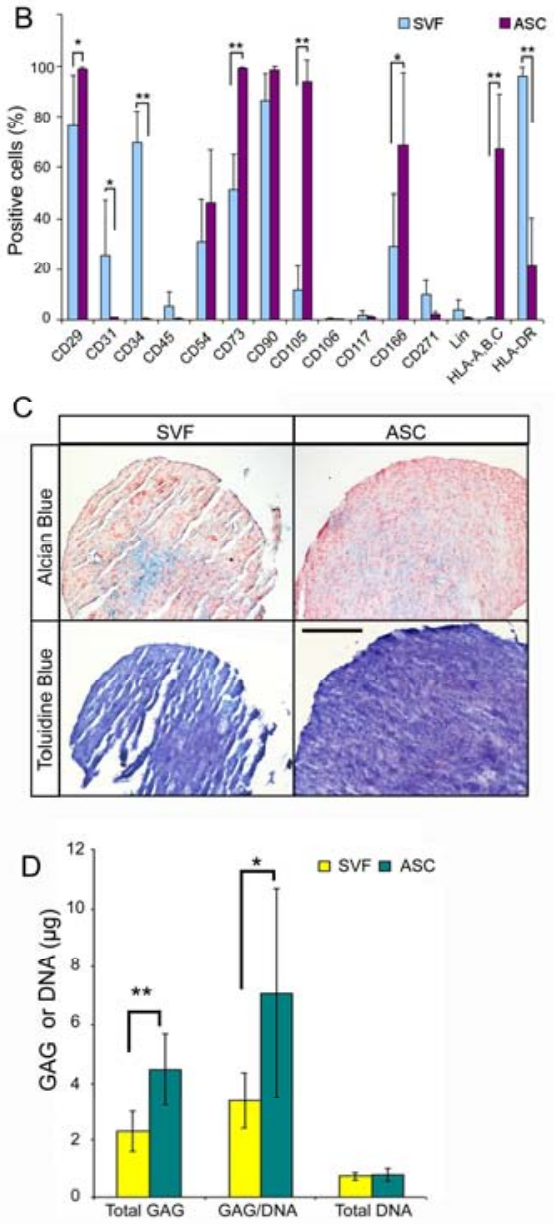

Figure 1 Comparison of surface marker profiles and chondrogenic potential between of SVF and ASC. (A) FACs was performed to analyse the profile the expression of CD markers on SVF and ASCs. Histograms from one representative donor pair of SVF and ASC are shown. Lines are isotypes, filled graphs indicate specific binding of antibodies. Of each cell fraction at least 100.000 cells were analyzed. (B) \% of positive cells for each CD marker was calculated based on the average of 9 donor pairs of SVF and ASC. Asterisk represents $P<0.05$. Double asterisk represents $P<0.01$. NS=Not Significant. Error bar reflects Standard Deviation (S. D.). Statistic analysis is done by student's t-test. (C) Chondrogenic potential of SVF and ASC is illustrated by histological staining. 200000 cells of SVF or ASC were seeded per well in a round botton nonadherent 96 -well plate. Cell pellets were made by centrifuge at 500g for $3 \mathrm{~min}$. Then pellets were cultured in chondrogenic differentiation medium for 4 weeks. Scale bar $=100 \mu \mathrm{m}$. Results of a representative donor pair of which SVF formed a stable pellet is shown. (D) At 4 weeks of chondrogenic differentiation GAG and DNA in the cell pellets were quantified. Data represent the average of 5 donor pairs of SVF, which formed stable pellets, and ASC. Asterisk represents $P<0.05$. Double asterisk represents $P<0.01$. Error bar reflects Standard Deviation (S. D.). Statistic analysis is done by student's t-test. 
SVFs expressed CD90; the levels of CD29, CD166, and CD105 were intermediate to these values. After isolation of ASCs by expansion in vitro, the percentage of cells staining positive for each of these markers increased. The initial SVF also contained a subpopulation of cells positive for stem cell-associated markers. An average of $69 \%$ of the SVFs expressed the HSC-associated marker CD34. Its level declined to $0.4 \%$ in ASCs. Expressions of CD73 increased from approximately 50\% in SVF to nearly $100 \%$ in ASCs. In general, ASC contained a more homogenous cell population which was CD31, CD34 and CD45 negative, but positive for MSC markers like CD105 and CD166. In line with our expectation, SVF represented a heterogeneous cell population containing not only cells with typical MSC characteristics but also CD31+ positive endothelial progenitors, CD45+ monocytes and CD34+ hematopoietic progenitors.

Then, the chondrogenic differentiation potential of SVF and ASC were characterized by putting them in pellet culture. ASC generally formed better aggregates than SVF. ASC from 9 donors all formed stable pellets, while stable pellet formation was only observed using SVF from 6 out of 9 donors. Results from histology and GAG quantification (figure 1C and D) showed that pellets from ASC are bigger and produce more cartilage matrix than SVF.

\section{Chondrocytes co-cultured with SVF produce more GAG than with ASC.}

To compare the effect of ASC on chondrocyte pellet co-cultures with SVF derived from the same donor, both cell fractions were co-cultured with primary human chondrocytes $(\mathrm{P} 2)$ in pellets $(\mathrm{SVF}+\mathrm{CH}$ and $\mathrm{ASC}+\mathrm{CH})$ in a ratio of $80 / 20$. Monocultures of chondrocytes $(\mathrm{CH})$ served as control. Three chondrocyte donors were randomly matched to $9 \mathrm{SVF}$ or ASC donor pairs. Results of histology from one representative experiment are shown in Figure 2 A. In contrast to pellet mono-cultures, in combination with primary human chondrocytes SVF of all 9 donors formed stable cell pellets. Alcian blue and Toluidine blue staining indicated the presence of GAG in all experimental groups. The staining appeared more intense in the $\mathrm{SVF}+\mathrm{CH}$ group. The presence of collagen type II was confirmed using immunohistochemistry. Overviews of these staining on whole pellets are shown in supplementary figure S 1A. Previously we showed that GAGs were mainly produced by chondrocytes in co-culture pellets $[15$, 
17]. Therefore, values of total GAG and GAG/DNA in co-culture groups were expressed relative to values obtained in mono-cultures of chondrocytes $(\mathrm{CH})$ groups. Data from $9 \mathrm{SVF}$ or ASC donors were averaged and displayed in figure $2 \mathrm{~B}$. SVF+CH group significantly showed higher capacity in producing GAG than the ASC $+\mathrm{CH}$ group. Expression of chondrogenic genes at week 4 of co-culture were examined by qPCR (Fig. 2C-E). Expression of collagen type 2 and aggrecan mRNA tended to be higher in the $\mathrm{SVF}+\mathrm{CH}$ group but this did not reach significance. Remarkably, co-culture of primary human chondrocytes with either the SVF or ASC cell fraction potently inhibited the expression of collagen type 10 mRNA, a marker for hypertrophic differentiation.

\section{Co-culture of chondrocytes with SVF and ASC induces chondrocyte proliferation}

EdU (5-ethynyl-2'-deoxyuridine) incorporation and cell tracking were used to investigate cell proliferation in the co-culture pellets. At day 2 after cell seeding EDU positive cells were detected in all groups (Fig. 2F left panel). Quantitative data are shown in figure $2 \mathrm{G}$ and figure $2 \mathrm{H}$. Percentages of EdU positive chondrocytes in coculture pellets tended to be higher in co-culture pellets with SVF than with ASC but this did not reach significance most likely due to large interdonor variation (Fig. 2G). After 2 days of culture, the percentage of EdU positive SVF or ASC cells in co-culture pellets did also not differ (Fig. 2H).

Previously we have shown that co-culture of primary chondrocytes with bone marrow MSCs induces massive cell death of the latter [30]. Therefore, a fluorescent TUNEL assay was performed to determine apoptotic cells in co-culture pellets at week 1. High numbers of TUNEL positive cells were found in cell pellets containing SVF or ASC, but very few in pellet mono-cultures of chondrocytes (Figure 2F right panel). TUNEL staining was significantly higher in SVF co-culture pellets than in ASC coculture pellets (Fig. 2I). After 4 weeks of co-culture, STR analysis was performed to determine the ratio of cells derived from chondrocytes or from either SVF or ASC donors. The distinguishable loci between chondrocytes and SVF/ASC donors were selected and averaged. In SVF co-culture pellets the initial seeding ratio of $80 \%$ SVF and $20 \%$ chondrocytes was changed to almost $60 \%$ chondrocytes and $40 \%$ SVF after 4 


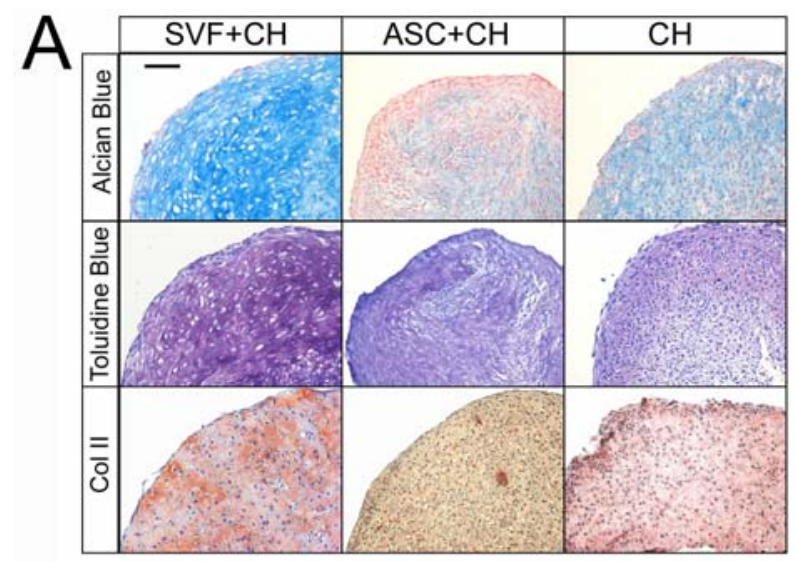

B
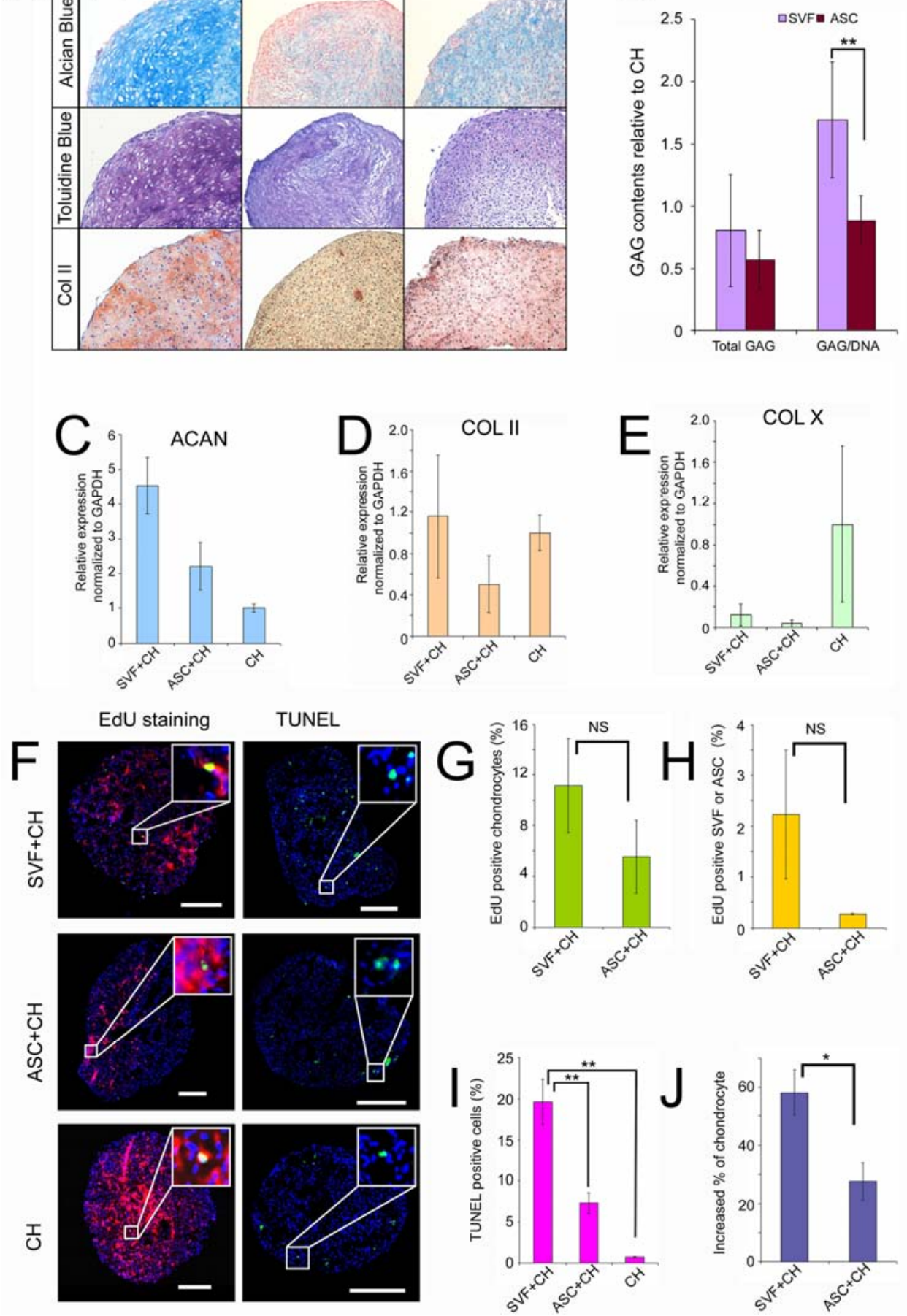
Figure 2 Co-culture of chondrocytes with SVF or ASC enhanced cartilage matrix formation; and induces apoptosis of MSCs and proliferation of chondrocytes. (A) Alcian blue and Toluidine blue staining indicated the presence of GAGs. Imnumohistochemical staining showed expression of Collagen type II. Cell pellets were cultured in chondrogenic differentiation medium (as described in Materials and Methods) for 4 weeks before examination. Scale Bar=100 $\mu \mathrm{m}$. Results of a representative a donor pair is shown. (B) GAG quantification showed that the $\mathrm{SVF}+\mathrm{CH}$ group produced more $\mathrm{GAG}$ than the $\mathrm{ASC}+\mathrm{CH}$ group. Data represent the average of 9 donor pairs of SVF (or ASC) with chondrocytes. Values are relative to pellet monoculture of chondrocytes $(\mathrm{CH})$. $P$ value was calculated by student's t-test. Error bar reflects Standard Deviation (S. D.). (C-E) Expression levels of $A C A N$ (C), COL2 (D) and COL9 (E) mRNA were examined by qPCR. RNA samples were extracted from 9 donor pairs of SVF and ASC with chondrocytes. from pellets cultured in chondrocyte proliferation medium for 4 weeks. RNA was isolated from 3 pellet cultures per condition. Relative expression levels were obtained by normalization to GAPDH. Values represent the relative gene expression compared to monoculture of chondrocytes (CH). Error bar reflects Standard Deviation (S. D.). (F) Left panel: EdU staining of pellets at day 2 after seeding. PCs were labeled with CM-DiI (red). EdU incorporation into newly synthesized DNA was visualized by Alexa 488 (green). Nuclei were counterstained with Hoechst 33342 (blue). Right panel: TUNEL staining of pellets were performed at 1 week after seeding. TUNEL positive cells were visualized with fluorescent labeling (green). Nuclei were counterstained with Hoechst 33342 (blue). Scale bar=100 $\mu \mathrm{m}$. A representative picture of 1 ASC or SVF donor is shown. (G) Quantification of EdU positive chondrocytes. Three pellets for each donor pair of SVF or ASC and chondrocytes were analyzed. Data represents the average of 3 donor pairs. Values expressed relative to monoculture of chondrocytes. Asterisk represents $P<0.05$. Double asterisk represents $P<0.01$. NS=Not Significant. Error bar reflects Standard Deviation (S. D.). (H) Quantification of EdU positive SVF or ASC. Three pellets for each donor pair of SVF or ASC and chondrocytes were analyzed. Data represents the average of 3 donor pairs. Asterisk represents $P<0.05$. Double asterisk represents $P<0.01$. NS=Not Significant. Error bar reflects Standard Deviation (S. D.). (I) Quantification of TUNEL positive cells. Three pellets for each donor pair of SVF or ASC and chondrocytes were analyzed. Data represents the average of 3 donor pairs. Asterisk represents $P<0.05$. Double asterisk represents $P<0.01$. NS=Not Significant. Error bar reflects Standard Deviation (S. D.). (J) STR analysis was performed on genomic DNA isolated from co-culture pellets of SVF or ASC and chondrocytes at an initial seeding ratio of 80:20 after 4 weeks of culture. Only informative loci that could discriminate between the SVF or ASC donor and PCs were taken into account. Averages of these loci were calculated to indicate the ratio of SVF or ASC and chondrocytes after co-culture. Data represents an average of 3 donor pairs. Asterisk represents $P<0.05$. Error bar reflects Standard Deviation (S. D.).

weeks of culture. The seeding ratio in the ASC co-culture pellets after 4 weeks of culture (75\% ASC and 25\% chondrocytes) did not significantly deviate from the initial seeding ratio (80\% ASC and 20\% chondrocytes) (Figure. 2J). Increased cell death of SVF cell fractions in combination with a larger stimulatory effect on chondrocyte 
proliferation are most likely responsible for the relative overgrowth of the primary chondrocytes in the co-culture pellets with SVF.

\section{Comparison of global gene expression profiles between SVF and ASC}

We have shown that trophic factors of bone marrow derived MSCs are responsible for increased chondrocyte proliferation and matrix deposition in co-culture pellets with chondrocytes. We therefore compared the global gene expression profile of pairs of SVF and ASCs to identify differentially expressed genes between both cell populations using a microarray experiment. As shown in Fig. 3A, the majorities of genes that can be detected in either cell type fall in the region of two fold change (up or down, yellow dots). 80 genes were found $>2$-fold up-regulated (red dots) in SVF while 48 genes were $>2$-fold down-regulated (blue dots). Differential expression of a random selection of genes was validated using qPCR (fig 3 B and C). Some variation in absolute fold change as determined by microarray or qPCR was observed, however the trends were the same. A complete list is given in supplementary table T2 showing all up-regulated and down-regulated genes with a more than 2-fold change and a $p$ value of $<0.05$ statistically analyzed by a one way ANOVA subsequent with a BenjaminiHochberg FDR correction and Tukey HSD post hoc test. As a result of the heterogeneous populations, genes higher expressed in SVF than in ASC are a combination of markers found in endothelial cells (e.g. EFNB2), adipocytes (e.g. APOD, FABP4) and lymphocytes (e.g. LTA, LITAF). On the other hand, ASC showed higher expressions of genes for mesenchymal cell lineages when compare to SVF. These genes included DKK3, TGFBI, GREM1 and SFRP2 which are expressed in chondrocytes[31-33], and COL1a1, SPARC and POSTN which are expressed in osteoblasts [34-35]. Roughly, one third of these differentially expressed genes are secreted factors, as shown in table 1. Some of the genes together with others (NFKB1 and UBD) on the top of the differentially expressed gene list are selected for qPCR validation (Fig. 3B and C). Variations between the data obtained from microarray and qPCR were observed, however the trends were the same.

The list of all up-regulated and down-regulated genes with more than 2-fold change was then imported into ingenuity pathway analysis software to identify 
Table 1 Differentially expressed secreted factors with more than 2 folds change

\begin{tabular}{|c|c|c|c|}
\hline Symbol & Accession & Fold change ( ASC vs SVF) & Reference \\
\hline APOD & NM_001647.2 & -6.43455 & $(46)$ \\
\hline PLA2G7 & NM_005084.2 & -5.71599 & $(47)$ \\
\hline EFNB2 & NM_004093.2 & -4.92421 & $(48)$ \\
\hline CXCL5 & NM_002994.3 & -4.32704 & $(49)$ \\
\hline FABP4 & NM_001442.1 & -3.32248 & $(50)$ \\
\hline LTB & NM_002341.1 & -2.79883 & $(51)$ \\
\hline CFD & NM_001928.2 & -2.55915 & $(52)$ \\
\hline THBD & NM_000361.2 & -2.55425 & (53) \\
\hline IL32 & NM_001012636.1 & -2.48646 & $(54)$ \\
\hline LITAF & NM_004862.2 & -2.37264 & $(55)$ \\
\hline FABP3 & NM_004102.3 & -2.2869 & $(56)$ \\
\hline NID1 & NM_002508.2 & -2.19089 & $(57)$ \\
\hline APOC1 & NM_001645.3 & -2.15423 & $(58)$ \\
\hline LTA & NM_000595.2 & -2.14469 & $(59)$ \\
\hline ATF3 & NM_001040619.1 & -2.13038 & $(60)$ \\
\hline IGFBP7 & NM_001553.1 & 2.019207 & $(61)$ \\
\hline BGN & NM_001711.3 & 2.20144 & $(62)$ \\
\hline IGFBP6 & NM_002178.2 & 2.260575 & $(63)$ \\
\hline FSTL1 & NM_007085.3 & 2.318603 & $(64)$ \\
\hline ADAM19 & NM_033274.2 & 2.343654 & (65) \\
\hline FBLN1 & NM_006487.2 & 2.418595 & $(66)$ \\
\hline F3 & NM_001993.2 & 2.446623 & $(67)$ \\
\hline COL11A1 & NM_080629.2 & 2.50218 & $(68)$ \\
\hline SERPINF1 & NM_002615.4 & 2.655044 & (69) \\
\hline FBLN2 & NM_001998.2 & 2.798432 & $(70)$ \\
\hline EFEMP2 & NM_016938.2 & 2.833782 & $(71)$ \\
\hline LOXL3 & NM_032603.2 & 3.072924 & (72) \\
\hline FN1 & NM_212474.1 & 3.253216 & (73) \\
\hline SFRP2 & NM_003013.2 & 3.32327 & (74) \\
\hline COL5A2 & NM_000393.3 & 3.63136 & (75) \\
\hline $\mathrm{COL} 1 \mathrm{~A} 2$ & NM_000089.3 & 3.631591 & (76) \\
\hline COL1A1 & NM_000088.3 & 4.273946 & (76) \\
\hline TGFBI & NM_000358.1 & 4.396439 & (77) \\
\hline POSTN & NM_006475.1 & 5.025622 & $(30)$ \\
\hline DKK3 & NM_013253.4 & 5.166207 & (74) \\
\hline COL5A1 & NM_000093.3 & 5.221677 & (75) \\
\hline SPARC & NM_003118.2 & 5.568203 & $(78)$ \\
\hline GREM1 & NM_013372.5 & 5.671927 & (79) \\
\hline MFGE8 & NM_005928.1 & 5.955669 & $(80)$ \\
\hline
\end{tabular}



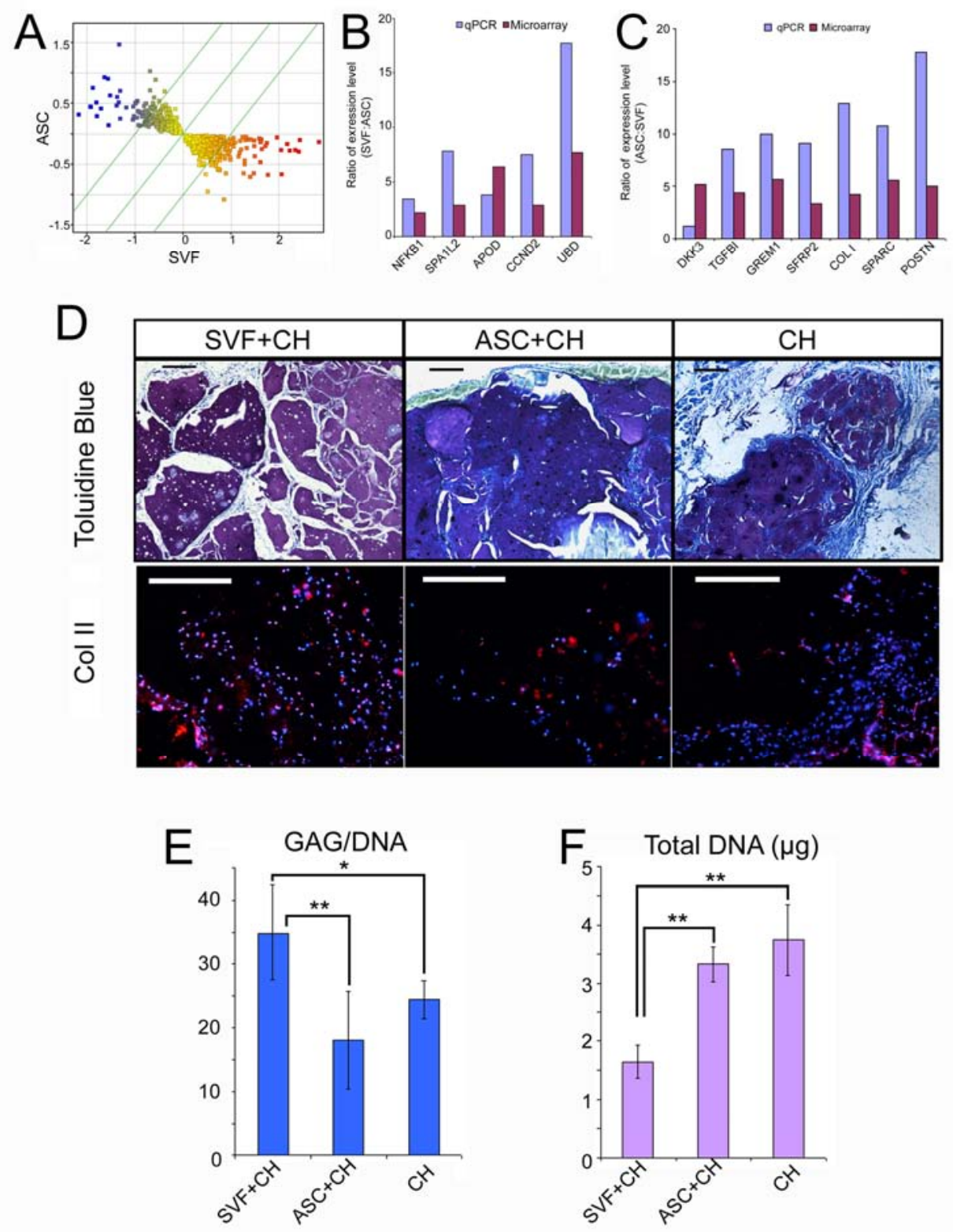

Figure 3 Comparison global gene expression profiles of SVF and ASC; and co-implantation chondrocytes with SVF produces more GAG than with ASC. (A) RNA samples were isolated from SVF or ASC of 3 donor pairs and applied in a microarray study. The average ratio of gene expression in SVF over ASC is plotted. Green lines indicate thresholds for up- ( $>2$ fold) and down-regulated gene expression ( $<2$ fold). In red 
are genes depicted which are $>2$ fold up regulated in SVF. In blue are genes depicted which are $>2$ fold downregulated in SVF. (B) Expression levels of genes that were up-regulated in SVF were validated by qPCR. The values are expressed relative to ASCs and represent the mean of 3 donors. (C) Expression levels of genes that are up-regulated in ASC were validated by qPCR. The values were relative to SVF and represent the mean of 3 donors. (D) SVF or ASC from 3 donors was pooled to eliminate donor variations, as well as chondrocytes from 3 donors. Then SVF or ASC were co-implanted with chondrocytes at a ratio of 4:1 into nude mice for 8 weeks. Constructs with chondrocyte only were served as positive control, while constructs without cells (blank) were used as negative control. Toluidine blue staining indicates the presence of GAGs. Immunohistochemical staining shows expression of Collagen type II. . Scale Bar=100 $\mu \mathrm{m}$. (E) GAG normalized to DNA in constructs. Amount of GAG and DNA of constructs $(\mathrm{N}=8)$ were measured by GAG and DNA assay. Values were subtracted by blank constructs to eliminate the influence of material on GAG assay. Asterisk represents $P<0.05$. Double asterisk represents $P<0.01$. NS=Not Significant. Error bar reflects Standard Deviation (S. D.). (F) Total DNA in TE constructs. Values were subtracted by blank constructs (without any cells) to eliminate the influence of material on GAG assay. Asterisk represents $P<0.05$. Double asterisk represents $P<0.01$. NS $=$ Not Significant. Error bar reflects Standard Deviation (S. D.).

differential expression between SVF and ASC in annotated canonical pathways and biofunctions. Significantly changed pathways were diseases and disorders (supplementary Fig. S2), molecular and cellular functions (supplementary Fig. S3) and physiological system development/function (supplementary Fig. S4). In addition, the differentially expressed genes were used as input into Search Tool for the Retrieval of Interacting Genes/Proteins to identify predicted interaction networks of genes and/or proteins (Supplementary Fig. S1B). The results show 3 majors clusters of interacting proteins. The first one (red) is a group of secreted factors centered by FN1 and TGFBI, most of which are produced by cells from mesenchymal lineage to regulate the differentiation and proliferation of chondrocytes. One cluster (brown) of extracellular matrix components centered by COL1A2 is also found. The third major cluster (yellow) is a group of transcription factors and cytokines which are regulated by NFKB1.

\section{Ectopic cartilage formation of SVF and ASC mixed with chondrocytes}

To evaluate our previous findings in vivo, mixtures of chondrocytes and SVF or ASC (ratio $20: 80$ ) were incorporated in an alginate gel and subcutaneously implanted in immunotolerant mice [36]. Eight weeks after implantation there was a significant accumulation of ECM components in the pericellular area in all experimental groups. SVF+CH group produced most ECM in comparison with the other two groups; 
however, the cartilage matrix is not mature according to the color of the staining (Fig. $3 \mathrm{D}$ upper panel). Immunofluorescent staining indicated the presence of more collagen type II protein expression in engineered cartilage of $\mathrm{SVF}+\mathrm{CH}$ than in (Fig. 3D lower panel). Results of GAG assay confirmed that $\mathrm{SVF}+\mathrm{CH}$ group contained more GAG than the other two groups (Fig. 3E). The quantification of DNA in the constructs showed that $\mathrm{SVF}+\mathrm{CH}$ had fewer cells (Fig. 3F). These data suggested that the SVF mixed with chondrocyte produced more cartilage matrix than ASC in vivo.

\section{Discussion}

In previous reports, we have shown that co-culture of MSCs and PC in pellets augmented cartilage matrix formation. This effect can be largely attributed to a trophic effect of the MSCs that increases the proliferation and matrix formation by chondrocytes, rather than by chondrogenic differentiation of MSCs. We furthermore showed that this trophic effect of MSCs is a general feature that can be observed in MSCs from multiple sources like bone marrow, adipose tissue and synovium [17]. In this study, we investigated for the first time the trophic effects of stromal vascular fraction of adipose tissue. We show that the SVF is a better cell source than ASC in facilitating cartilage formation in co-culture with chondrocytes. This conclusion is based on the following observations; i) Co-culture pellets of SVF and chondrocytes produced more GAG than pellets of ASC and chondrocytes, ii) the proportion of chondrocytes after co-culturing is more increased in co-culture pellets of SVF and chondrocytes than pellets of ASC and chondrocytes, and iii) mixtures of SVF and chondrocytes deposit more GAG and collagen type 2 than that of ASC and chondrocytes in an ectopic cartilage formation model in nude mice.

For the first time, we showed here that SVF cells could act as trophic mediators in co-culture pellets with chondrocytes. We previously reported that cultured ASCs (adipose derived mesenchymal stem cells) could induce chondrocyte proliferation in co-culture pellets and promoted GAG formation in co-cultures. In this study, we showed that in comparison to ASCs, SVF from the same donor were more potent in promoting GAG formation in co-cultures. The proliferation rate of chondrocytes tended to be higher in co-culture with SVF but this did not reach significance. This is likely 
explained by considerable inter-donor variation. However, we observed another interesting finding that more cells death was found in $\mathrm{SVF}+\mathrm{CH}$ than in $\mathrm{ASC}+\mathrm{CH}$ coculture pellets. This could possibly explain why average GAG production of each cell (GAG/DNA) is higher in $\mathrm{SVF}+\mathrm{CH}$ than in $\mathrm{ASC}+\mathrm{CH}$. Increased cell death possibly in combination with increased proliferation is likely responsible for the relative overgrowth of chondrocytes after 4 weeks of co-culture particularly in the SVF coculture group compared to the ASC co-culture group. This was confirmed by STR analysis.

Based on our results, SVF cells could be a very good source of cells used for cartilage regeneration. In previous studies, Jurgens et al reported that SVF cells showed stem cell characteristics that are very similar to cultured ASC, and SVF cells even appeared to be slightly better than cultured ASC in chondrogenic differentiation as indicated by GAG quantification [14]. In comparison with bone marrow derived MSCs, SVF cells also differentiate into osteogenic, adipogenic and chondrogenic lineages, but have a much higher Colony Form Unit (CFU) frequency[37]. It's also been reported that SVF cells could quickly attach to poly (L-lactide-co-caprolactone) and collagen type I/III scaffolds which are both suitable for cartilage tissue engineering [38]. Here we show data to suport that co-culture pellets of SVF cells and chondrocytes deposit more cartilage matrix than co-culture pellets of ASC and chondrocytes as well as monoculture of chondrocytes. Even after co-implantation of SVF or ASC cells in an alginate gel, more ectopic cartilage matrix formation is observed in the combination of SVF with chondrocytes after subcutaneous implantation in a nude mouse model. Since SVF can be relatively easy and fast isolated out of a liposuction, it is possible to isolate SVF and reimplant the cells into the patient in one-step surgery.

In marked contrast to ASCs, SVF contains a heterogeneous cell population [39]. It is believed that ASCs have a more homogeneous composition of cell types, even though ASC and SVF share some common features like multi-lineage differentiation. In addition to this, our data from FACs and Microarray indicated that ASC are more prior to mesenchymal cell lineages than SVF. This is in agreement with literature that SVF contains cell population of endothelial cell and hematopoietic cells besides 
mesenchymals stem cells [37]. With unknown mechanism, these non-mesenchymal cells enhanced the trophic effects of MSCs at least in co-culture with chondrocytes. There could be two hypotheses to explain this mechanism: 1) one or more of the nonMSCs populations is actually better trophic mediators than MSCs that enhance matrix formation of chondrocytes; 2) some of the non-MSC cells preserves/increase the trophic effects of MSCs. So far, we don't have enough data to exclude one of the above possibilities. However, pre-existing dada seems to support the second. It has been reported that ASCs resided in the adventitia of blood vessels as a group of CD34+/CD31-/ $\alpha$-smooth muscle actin- (smA) cells [40-42]. There are debates about the CD markers of these cells [43-44]; nevertheless, all these studies pointed that ASCs reside in a perivascular niche in situ. This niche or local microenvironment dominantly made of endothelial cells is of course important in maintaining ASC's phenotype as stem cells; meanwhile it may be also crucial for keeping their function as trophic mediators. It was believed that ASCs could stabilize endothelial networks in vitro and help vessel formation in vivo [45]. As a feedback, it's also possible that endothelial cells may better preserve some of ASC's characteristics which are lost during in vitro expansion, such as trophic effects.

In conclusion, our data demonstrated that chondrocytes form better cartilage tissue when co-cultured or co-implanted with SVF of adipose tissue than with ASC. Heterogeneous populations of SVF shows higher level of trophic effects in cartilage formation. These results support the clinical potential of one step therapy for cartilage repair, in which SVF from adipose tissue and chondrocytes from non-weight bearing joint surface are isolated, mixed and implanted back into the patient during the same surgical procedure. 


\section{References}

1. F. Dell'accio, T. L. Vincent, Joint surface defects: clinical course and cellular response in spontaneous and experimental lesions. Eur Cell Mater 20, 210-217 (2010).

2. S. L. Sledge, Microfracture techniques in the treatment of osteochondral injuries. Clin Sports Med 20, 365-377 (2001).

3. M. Brittberg, A. Lindahl, A. Nilsson, C. Ohlsson, O. Isaksson, L. Peterson, Treatment of deep cartilage defects in the knee with autologous chondrocyte transplantation. N Engl J Med 331, 889895 (1994).

4. J. W. Alford, B. J. Cole, Cartilage restoration, part 2: techniques, outcomes, and future directions. Am J Sports Med 33, 443-460 (2005).

5. H. Robert, Chondral repair of the knee joint using mosaicplasty. Orthop Traumatol Surg Res 97, 418-429 (2011).

6. P. Niemeyer, J. M. Pestka, P. C. Kreuz, C. Erggelet, H. Schmal, N. P. Suedkamp, M. Steinwachs, Characteristic complications after autologous chondrocyte implantation for cartilage defects of the knee joint. Am J Sports Med 36, 2091-2099 (2008).

7. G. Vadala, R. K. Studer, G. Sowa, F. Spiezia, C. Iucu, V. Denaro, L. G. Gilbertson, J. D. Kang, Coculture of bone marrow mesenchymal stem cells and nucleus pulposus cells modulate gene expression profile without cell fusion. Spine (Phila Pa 1976) 33, 870-876 (2008).

8. X. T. Mo, S. C. Guo, H. Q. Xie, L. Deng, W. Zhi, Z. Xiang, X. Q. Li, Z. M. Yang, Variations in the ratios of co-cultured mesenchymal stem cells and chondrocytes regulate the expression of cartilaginous and osseous phenotype in alginate constructs. Bone 45, 42-51 (2009).

9. K. Tsuchiya, G. Chen, T. Ushida, T. Matsuno, T. Tateishi, The effect of coculture of chondrocytes with mesenchymal stem cells on their cartilaginous phenotype in vitro. Materials Science \& Engineering C-Biomimetic and Supramolecular Systems 24, 6 (2004).

10. P. A. Zuk, M. Zhu, H. Mizuno, J. Huang, J. W. Futrell, A. J. Katz, P. Benhaim, H. P. Lorenz, M. H. Hedrick, Multilineage cells from human adipose tissue: implications for cell-based therapies. Tissue Eng 7, 211-228 (2001).

11. P. A. Zuk, M. Zhu, P. Ashjian, D. A. De Ugarte, J. I. Huang, H. Mizuno, Z. C. Alfonso, J. K. Fraser, P. Benhaim, M. H. Hedrick, Human adipose tissue is a source of multipotent stem cells. Mol Biol Cell 13, 4279-4295 (2002).

12. Y. Lin, L. Liu, Z. Li, J. Qiao, L. Wu, W. Tang, X. Zheng, X. Chen, Z. Yan, W. Tian, Pluripotency potential of human adipose-derived stem cells marked with exogenous green fluorescent protein. Mol Cell Biochem 291, 1-10 (2006).

13. M. J. Varma, R. G. Breuls, T. E. Schouten, W. J. Jurgens, H. J. Bontkes, G. J. Schuurhuis, S. M. van Ham, F. J. van Milligen, Phenotypical and functional characterization of freshly isolated adipose tissue-derived stem cells. Stem Cells Dev 16, 91-104 (2007).

14. W. J. Jurgens, A. van Dijk, B. Z. Doulabi, F. B. Niessen, M. J. Ritt, F. J. van Milligen, M. N. Helder, Freshly isolated stromal cells from the infrapatellar fat pad are suitable for a one-step surgical procedure to regenerate cartilage tissue. Cytotherapy 11, 1052-1064 (2009).

15. L. Wu, J. C. Leijten, N. Georgi, J. N. Post, C. A. van Blitterswijk, M. Karperien, Trophic effects of mesenchymal stem cells increase chondrocyte proliferation and matrix formation. Tissue Eng Part A 17, 1425-1436 (2011).

16. C. Acharya, A. Adesida, P. Zajac, M. Mumme, J. Riesle, I. Martin, A. Barbero, Enhanced chondrocyte proliferation and mesenchymal stromal cells chondrogenesis in coculture pellets mediate improved cartilage formation. J Cell Physiol, (2011).

17. L. Wu, H. J. Prins, M. Helder, C. van Blitterswijk, M. Karperien, Trophic effects of mesenchymal stem cells in chondrocyte co-cultures are independent of culture conditions and cell sources. Tissue Eng Part A, (2012).

18. M. Singer, Trophic functions of the neuron. VI. Other trophic systems. Neurotrophic control of limb regeneration in the newt. Ann N Y Acad Sci 228, 308-322 (1974).

19. A. I. Caplan, J. E. Dennis, Mesenchymal stem cells as trophic mediators. J Cell Biochem 98, 10761084 (2006).

20. S. P. Bruder, D. J. Fink, A. I. Caplan, Mesenchymal stem cells in bone development, bone repair, and skeletal regeneration therapy. J Cell Biochem 56, 283-294 (1994).

21. I. Kassis, A. Vaknin-Dembinsky, D. Karussis, Bone marrow mesenchymal stem cells: agents of immunomodulation and neuroprotection. Curr Stem Cell Res Ther 6, 63-68 (2011). 
22. Y. Li, J. Chen, C. L. Zhang, L. Wang, D. Lu, M. Katakowski, Q. Gao, L. H. Shen, J. Zhang, M. $\mathrm{Lu}, \mathrm{M}$. Chopp, Gliosis and brain remodeling after treatment of stroke in rats with marrow stromal cells. Glia 49, 407-417 (2005).

23. C. Sassoli, A. Pini, B. Mazzanti, F. Quercioli, S. Nistri, R. Saccardi, S. Z. Orlandini, D. Bani, L. Formigli, Mesenchymal stromal cells affect cardiomyocyte growth through juxtacrine Notch1/Jagged1 signaling and paracrine mechanisms: Clues for cardiac regeneration. J Mol Cell Cardiol, (2011).

24. Y. L. Tang, Q. Zhao, X. Qin, L. Shen, L. Cheng, J. Ge, M. I. Phillips, Paracrine action enhances the effects of autologous mesenchymal stem cell transplantation on vascular regeneration in rat model of myocardial infarction. Ann Thorac Surg 80, 229-236; discussion 236-227 (2005).

25. L. Wu, X. Cai, H. Dong, W. Jing, Y. Huang, X. Yang, Y. Wu, Y. Lin, Serum regulates adipogenesis of mesenchymal stem cells via MEK/ERK-dependent PPARgamma expression and phosphorylation. J Cell Mol Med 14, 922-932 (2010).

26. A. H. Huang, A. Stein, R. L. Mauck, Evaluation of the complex transcriptional topography of mesenchymal stem cell chondrogenesis for cartilage tissue engineering. Tissue Eng Part A 16, 2699-2708 (2010).

27. J. C. Leijten, C. A. van Blitterwijk, M. Karperien, J. Emons, S. van Gool, J. M. Wit, C. Sticht, E. Decker, G. Rappold, A. Uitterlinden, F. Rivadeneira, J. van Meurs, A. Hofman, S. Scherjon, GREM1, FRZB and DKK1 are key regulators of human articular cartilage homeostasis. Arthritis Rheum, (2012).

28. R. Morello, T. K. Bertin, S. Schlaubitz, C. A. Shaw, S. Kakuru, E. Munivez, P. Hermanns, Y. Chen, B. Zabel, B. Lee, Brachy-syndactyly caused by loss of Sfrp2 function. J Cell Physiol 217, 127-137 (2008).

29. K. Kapinas, K. M. Lowther, C. B. Kessler, K. Tilbury, J. R. Lieberman, J. S. Tirnauer, P. Campagnola, A. M. Delany, Bone matrix osteonectin limits prostate cancer cell growth and survival. Matrix Biol 31, 299-307 (2012).

30. S. M. Xiao, Y. Gao, C. L. Cheung, C. H. Bow, K. S. Lau, P. C. Sham, K. C. Tan, A. W. Kung, Association of CDX1 binding site of periostin gene with bone mineral density and vertebral fracture risk. Osteoporos Int 23, 1877-1887 (2012).

31. Y. Lin, E. Luo, X. Chen, L. Liu, J. Qiao, Z. Yan, Z. Li, W. Tang, X. Zheng, W. Tian, Molecular and cellular characterization during chondrogenic differentiation of adipose tissue-derived stromal cells in vitro and cartilage formation in vivo. J Cell Mol Med 9, 929-939 (2005).

32. G. Astori, F. Vignati, S. Bardelli, M. Tubio, M. Gola, V. Albertini, F. Bambi, G. Scali, D. Castelli, V. Rasini, G. Soldati, T. Moccetti, "In vitro" and multicolor phenotypic characterization of cell subpopulations identified in fresh human adipose tissue stromal vascular fraction and in the derived mesenchymal stem cells. J Transl Med 5, 55 (2007).

33. W. J. Jurgens, R. J. Kroeze, R. A. Bank, M. J. Ritt, M. N. Helder, Rapid attachment of adipose stromal cells on resorbable polymeric scaffolds facilitates the one-step surgical procedure for cartilage and bone tissue engineering purposes. J Orthop Res 29, 853-860 (2011).

34. J. M. Gimble, W. Grayson, F. Guilak, M. J. Lopez, G. Vunjak-Novakovic, Adipose tissue as a stem cell source for musculoskeletal regeneration. Front Biosci (Schol Ed) 3, 69-81 (2011).

35. C. S. Lin, Z. C. Xin, C. H. Deng, H. Ning, G. Lin, T. F. Lue, Defining adipose tissue-derived stem cells in tissue and in culture. Histol Histopathol 25, 807-815 (2010).

36. L. Zimmerlin, V. S. Donnenberg, M. E. Pfeifer, E. M. Meyer, B. Peault, J. P. Rubin, A. D. Donnenberg, Stromal vascular progenitors in adult human adipose tissue. Cytometry A 77, 22-30 (2010).

37. M. Crisan, S. Yap, L. Casteilla, C. W. Chen, M. Corselli, T. S. Park, G. Andriolo, B. Sun, B. Zheng, L. Zhang, C. Norotte, P. N. Teng, J. Traas, R. Schugar, B. M. Deasy, S. Badylak, H. J. Buhring, J. P. Giacobino, L. Lazzari, J. Huard, B. Peault, A perivascular origin for mesenchymal stem cells in multiple human organs. Cell Stem Cell 3, 301-313 (2008).

38. A. C. Zannettino, S. Paton, A. Arthur, F. Khor, S. Itescu, J. M. Gimble, S. Gronthos, Multipotential human adipose-derived stromal stem cells exhibit a perivascular phenotype in vitro and in vivo. $J$ Cell Physiol 214, 413-421 (2008).

39. X. Cai, Y. Lin, P. V. Hauschka, B. E. Grottkau, Adipose stem cells originate from perivascular cells. Biol Cell 103, 435-447 (2011).

40. D. O. Traktuev, D. N. Prater, S. Merfeld-Clauss, A. R. Sanjeevaiah, M. R. Saadatzadeh, M. Murphy, B. H. Johnstone, D. A. Ingram, K. L. March, Robust functional vascular network 
formation in vivo by cooperation of adipose progenitor and endothelial cells. Circ Res 104, 14101420 (2009).

41. J. Hendriks, J. Riesle, C. A. Vanblitterswijk, Effect of stratified culture compared to confluent culture in monolayer on proliferation and differentiation of human articular chondrocytes. Tissue Eng 12, 2397-2405 (2006).

42. W. J. Jurgens, M. J. Oedayrajsingh-Varma, M. N. Helder, B. Zandiehdoulabi, T. E. Schouten, D. J. Kuik, M. J. Ritt, F. J. van Milligen, Effect of tissue-harvesting site on yield of stem cells derived from adipose tissue: implications for cell-based therapies. Cell Tissue Res 332, 415-426 (2008).

43. S. Y. Lee, T. Nakagawa, A. H. Reddi, Mesenchymal progenitor cells derived from synovium and infrapatellar fat pad as a source for superficial zone cartilage tissue engineering: analysis of superficial zone protein/lubricin expression. Tissue Eng Part A 16, 317-325 (2010).

44. K. J. Livak, T. D. Schmittgen, Analysis of relative gene expression data using real-time quantitative PCR and the 2(-Delta Delta C(T)) Method. Methods 25, 402-408 (2001).

45. D. Szklarczyk, A. Franceschini, M. Kuhn, M. Simonovic, A. Roth, P. Minguez, T. Doerks, M. Stark, J. Muller, P. Bork, L. J. Jensen, C. von Mering, The STRING database in 2011: functional interaction networks of proteins, globally integrated and scored. Nucleic Acids Res 39, D561-568 (2011).

46. J. Kosacka, M. Gericke, M. Nowicki, J. Kacza, J. Borlak, K. Spanel-Borowski, Apolipoproteins D and E3 exert neurotrophic and synaptogenic effects in dorsal root ganglion cell cultures. Neuroscience 162, 282-291 (2009).

47. J. Chen, L. Yang, J. M. Foulks, A. S. Weyrich, G. K. Marathe, T. M. McIntyre, Intracellular PAF catabolism by PAF acetylhydrolase counteracts continual PAF synthesis. J Lipid Res 48, 2365 2376 (2007).

48. H. Luo, Z. Wu, S. Qi, W. Jin, B. Han, J. Wu, Ephrinb1 and Ephrinb2 are associated with interleukin-7 receptor alpha and retard its internalization from the cell surface. J Biol Chem 286, 44976-44987 (2011).

49. S. L. Zhou, Z. Dai, Z. J. Zhou, X. Y. Wang, G. H. Yang, Z. Wang, X. W. Huang, J. Fan, J. Zhou, Overexpression of CXCL5 mediates neutrophil infiltration and indicates poor prognosis for hepatocellular carcinoma. Hepatology, (2012).

50. M. Furuhashi, S. Ishimura, H. Ota, M. Hayashi, T. Nishitani, M. Tanaka, H. Yoshida, K. Shimamoto, G. S. Hotamisligil, T. Miura, Serum fatty acid-binding protein 4 is a predictor of cardiovascular events in end-stage renal disease. PLoS One 6, e27356 (2011).

51. V. R. Narala, R. K. Adapala, M. V. Suresh, T. G. Brock, M. Peters-Golden, R. C. Reddy, Leukotriene B4 is a physiologically relevant endogenous peroxisome proliferator-activated receptor-alpha agonist. J Biol Chem 285, 22067-22074 (2010).

52. E. Kitano, H. Kitamura, Synthesis of factor D by gastric cancer-derived cell lines. Int Immunopharmacol 2, 843-848 (2002).

53. S. Ida, Y. Fujimura, M. Hirota, Y. Imamura, N. Ozaki, K. Suyama, D. Hashimoto, M. Ohmuraya, H. Tanaka, H. Takamori, H. Baba, Significance of endothelial molecular markers in the evaluation of the severity of acute pancreatitis. Surg Today 39, 314-319 (2009).

54. J. Choi, S. Bae, J. Hong, S. Ryoo, H. Jhun, K. Hong, D. Yoon, S. Lee, E. Her, W. Choi, J. Kim, T. Azam, C. A. Dinarello, S. Kim, Paradoxical effects of constitutive human IL-32\{gamma in transgenic mice during experimental colitis. Proc Natl Acad Sci U S A 107, 21082-21086 (2010).

55. A. Gazel, M. Rosdy, C. Tornier, B. De Fraissinette Ade, M. Blumenberg, Transcriptional profiling defines the effects of nickel in human epidermal keratinocytes. J Cell Physiol 217, 686-692 (2008).

56. V. Lamounier-Zepter, C. Look, J. Alvarez, T. Christ, U. Ravens, W. H. Schunck, M. EhrhartBornstein, S. R. Bornstein, I. Morano, Adipocyte fatty acid-binding protein suppresses cardiomyocyte contraction: a new link between obesity and heart disease. Circ Res 105, 326-334 (2009).

57. A. Furuyama, T. Hosokawa, K. Mochitate, Interleukin-1beta and tumor necrosis factor-alpha have opposite effects on fibroblasts and epithelial cells during basement membrane formation. Matrix Biol 27, 429-440 (2008).

58. R. X. Li, Y. B. Ding, S. L. Zhao, Y. Y. Xiao, Q. R. Li, F. Y. Xia, L. Sun, X. Lin, J. R. Wu, K. Liao, R. Zeng, Secretome-derived isotope tags (SDIT) reveal adipocyte-derived apolipoprotein C-I as a predictive marker for cardiovascular disease. J Proteome Res 11, 2851-2862 (2012).

59. X. S. Zhang, G. B. Proctor, J. R. Garrett, B. A. Schulte, D. K. Shori, Use of lectin probes on tissues and sympathetic saliva to study the glycoproteins secreted by rat submandibular glands. $J$ Histochem Cytochem 42, 1261-1269 (1994). 
60. X. Yin, J. W. Dewille, T. Hai, A potential dichotomous role of ATF3, an adaptive-response gene, in cancer development. Oncogene 27, 2118-2127 (2008).

61. N. Alic, M. P. Hoddinott, G. Vinti, L. Partridge, Lifespan extension by increased expression of the Drosophila homologue of the IGFBP7 tumour suppressor. Aging Cell 10, 137-147 (2011).

62. A. D. Berendsen, L. W. Fisher, T. M. Kilts, R. T. Owens, P. G. Robey, J. S. Gutkind, M. F. Young, Modulation of canonical Wnt signaling by the extracellular matrix component biglycan. Proc Natl Acad Sci U S A 108, 17022-17027 (2011).

63. S. J. Freemantle, J. S. Kerley, S. L. Olsen, R. H. Gross, M. J. Spinella, Developmentally-related candidate retinoic acid target genes regulated early during neuronal differentiation of human embryonal carcinoma. Oncogene 21, 2880-2889 (2002).

64. J. Xu, X. Qi, J. Gong, M. Yu, F. Zhang, H. Sha, X. Gao, Fstll antagonizes BMP signaling and regulates ureter development. PLoS One 7, e32554 (2012).

65. C. Bret, D. Hose, T. Reme, A. Kassambara, A. Seckinger, T. Meissner, J. F. Schved, T. Kanouni, H. Goldschmidt, B. Klein, Gene expression profile of ADAMs and ADAMTSs metalloproteinases in normal and malignant plasma cells and in the bone marrow environment. Exp Hematol 39, 546557 e548 (2011).

66. P. Debeer, E. F. Schoenmakers, W. O. Twal, W. S. Argraves, L. De Smet, J. P. Fryns, W. J. Van De Ven, The fibulin-1 gene (FBLN1) is disrupted in a $t(12 ; 22)$ associated with a complex type of synpolydactyly. J Med Genet 39, 98-104 (2002).

67. N. Hahn, M. Heiden, R. Seitz, U. Salge-Bartels, Inducible expression of tissue factor in small-cell lung cancer: impact on morphology and matrix metalloproteinase secretion. J Cancer Res Clin Oncol 138, 695-703 (2012).

68. R. P. Kirwan, R. J. Wordinger, A. F. Clark, C. J. O'Brien, Differential global and extra-cellular matrix focused gene expression patterns between normal and glaucomatous human lamina cribrosa cells. Mol Vis 15, 76-88 (2009).

69. J. Becker, O. Semler, C. Gilissen, Y. Li, H. J. Bolz, C. Giunta, C. Bergmann, M. Rohrbach, F. Koerber, K. Zimmermann, P. de Vries, B. Wirth, E. Schoenau, B. Wollnik, J. A. Veltman, A. Hoischen, C. Netzer, Exome sequencing identifies truncating mutations in human SERPINF1 in autosomal-recessive osteogenesis imperfecta. Am J Hum Genet 88, 362-371 (2011).

70. E. Ducros, A. Berthaut, P. Mirshahi, S. Lemarchand, J. Soria, J. M. Legeais, M. Mirshahi, Expression of extracellular matrix proteins fibulin-1 and fibulin-2 by human corneal fibroblasts. Curr Eye Res 32, 481-490 (2007).

71. L. Yao, W. Lao, Y. Zhang, X. Tang, X. Hu, C. He, L. X. Xu, Identification of EFEMP2 as a Serum Biomarker for the Early Detection of Colorectal Cancer with Lectin Affinity Capture Assisted Secretome Analysis of Cultured Fresh Tissues. J Proteome Res, (2012).

72. J. M. Maki, K. I. Kivirikko, Cloning and characterization of a fourth human lysyl oxidase isoenzyme. Biochem J 355, 381-387 (2001).

73. N. Wu, J. Rollin, I. Masse, J. Lamartine, X. Gidrol, p63 regulates human keratinocyte proliferation via MYC-regulated gene network and differentiation commitment through cell adhesion-related gene network. J Biol Chem 287, 5627-5638 (2012).

74. S. M. Hauck, F. Hofmaier, J. Dietter, M. E. Swadzba, M. Blindert, B. Amann, J. Behler, E. Kremmer, M. Ueffing, C. A. Deeg, Label-free LC-MSMS analysis of vitreous from autoimmune uveitis reveals a significant decrease in secreted Wnt signalling inhibitors DKK3 and SFRP2. $J$ Proteomics, (2012).

75. S. Symoens, F. Malfait, P. Vlummens, T. Hermanns-Le, D. Syx, A. De Paepe, A novel splice variant in the N-propeptide of COL5A1 causes an EDS phenotype with severe kyphoscoliosis and eye involvement. PLoS One 6, e20121 (2011).

76. C. D. Constantinou, M. Pack, S. B. Young, D. J. Prockop, Phenotypic heterogeneity in osteogenesis imperfecta: the mildly affected mother of a proband with a lethal variant has the same mutation substituting cysteine for alpha 1-glycine 904 in a type I procollagen gene (COL1A1). Am J Hum Genet 47, 670-679 (1990).

77. D. A. Tumbarello, J. Temple, J. D. Brenton, Beta3 integrin modulates transforming growth factor beta induced (TGFBI) function and paclitaxel response in ovarian cancer cells. Mol Cancer 11, 36 (2012).

78. P. Bhoopathi, B. Gorantla, G. S. Sailaja, C. S. Gondi, M. Gujrati, J. D. Klopfenstein, J. S. Rao, SPARC overexpression inhibits cell proliferation in neuroblastoma and is partly mediated by tumor suppressor protein PTEN and AKT. PLoS One 7, e36093 (2012). 
79. O. Michos, A. Goncalves, J. Lopez-Rios, E. Tiecke, F. Naillat, K. Beier, A. Galli, S. Vainio, R. Zeller, Reduction of BMP4 activity by gremlin 1 enables ureteric bud outgrowth and GDNF/WNT11 feedback signalling during kidney branching morphogenesis. Development 134, 2397-2405 (2007).

80. S. Motegi, W. W. Leitner, M. Lu, Y. Tada, M. Sardy, C. Wu, T. Chavakis, M. C. Udey, Pericytederived MFG-E8 regulates pathologic angiogenesis. Arterioscler Thromb Vasc Biol 31, 2024-2034 (2011). 


\section{Supplementary Materials}
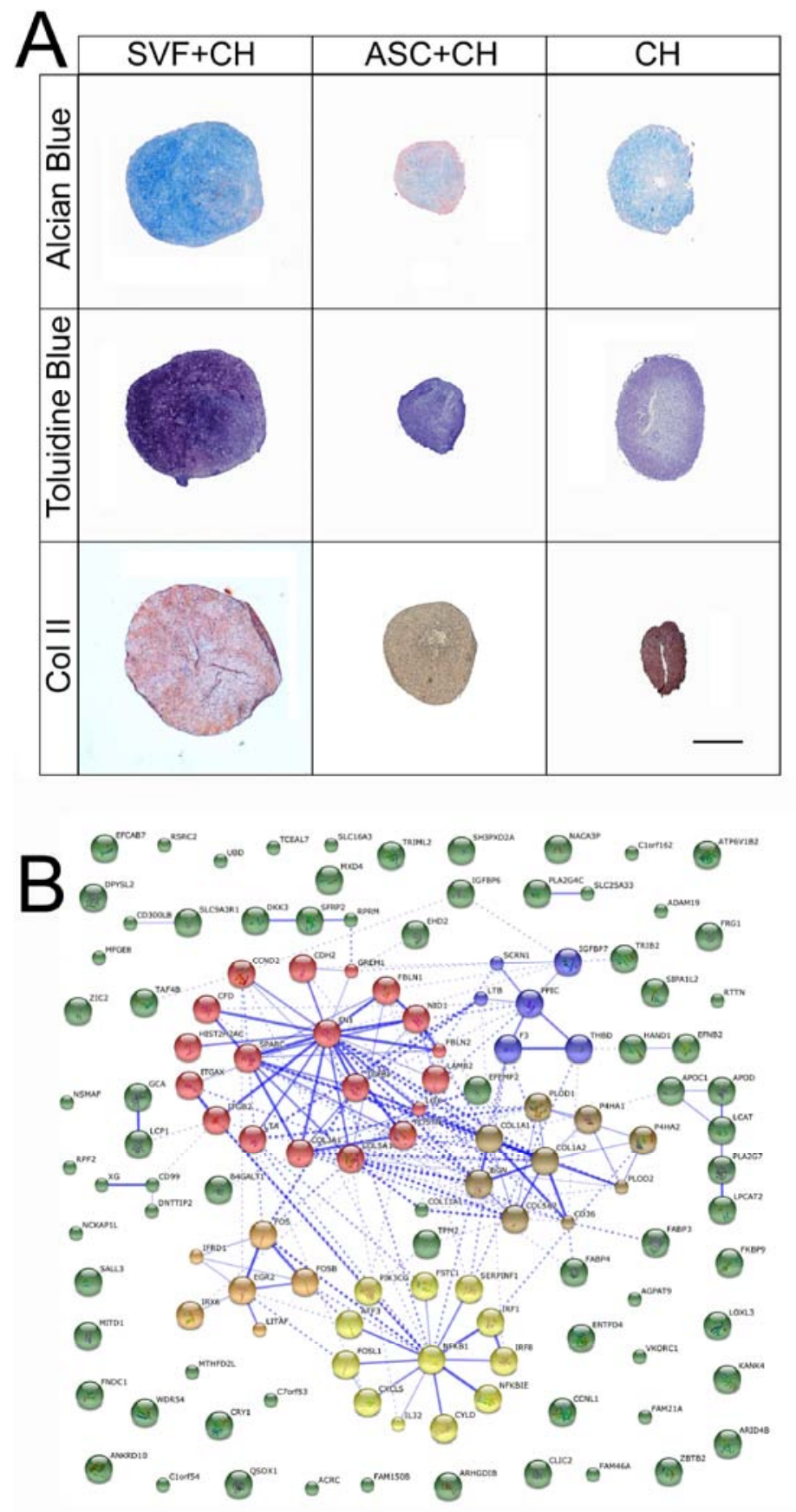

Figure S1 (A) Overview of co-culture pellets $(\mathrm{SVF}+\mathrm{CH}$ and $\mathrm{ASC}+\mathrm{CH})$ and mono-culture pellets (CH) from one representative donor pair. Pellets were cultured in chondrogenic medium (as described in Materials and Methods) for 4 weeks before processing for histology. Images of Alcian blue, Toluidine blue and immunohistochemical (antibody against COL2) staining were shown. Bar $=500 \mu \mathrm{m}$. CH stands for monoculture of chondrocytes. (B) Possible interaction networks of genes/proteins of upregulated genes. The predicted interaction networks of the 128 up/down-regulated genes/proteins were studied by the Search Tool for the Retrieval of Interacting Genes/Proteins as described in materials and methods. 


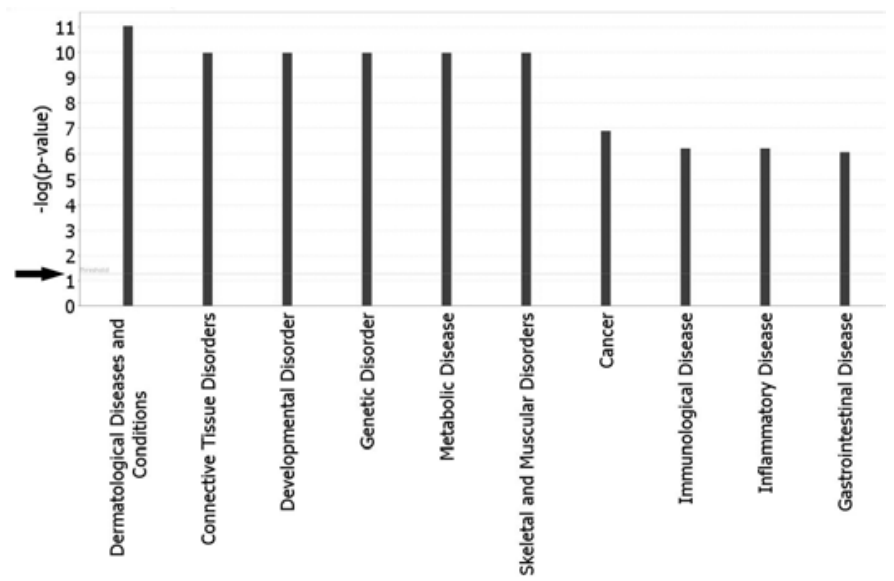

Figure S2. The list of 128 up/down-regulated genes was imported into ingenuity pathway analysis software to visualize the changes of gene expression in Diseases and Disorders pathways. Top-ten pathways that changed most were listed. Arrow indicates threshold of significance.

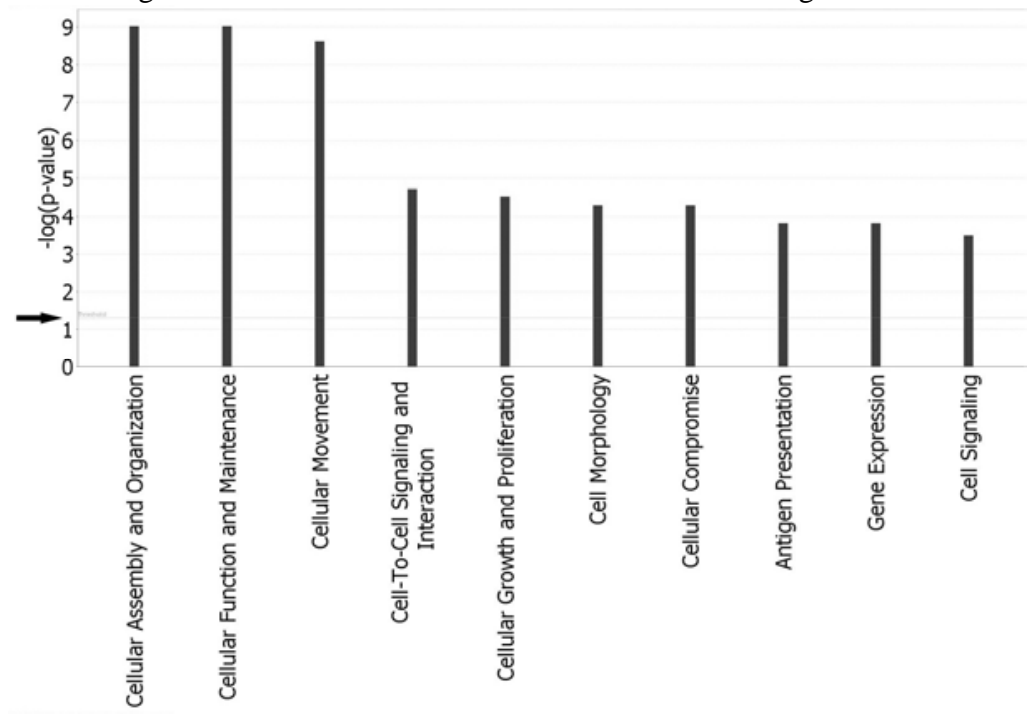

Figure S3. Changes of gene expression in Molecular and Cellular Functions pathway were analysis the same way as in Fig S3. Top-ten pathways that changed most were listed. Arrow indicates threshold of significance. 


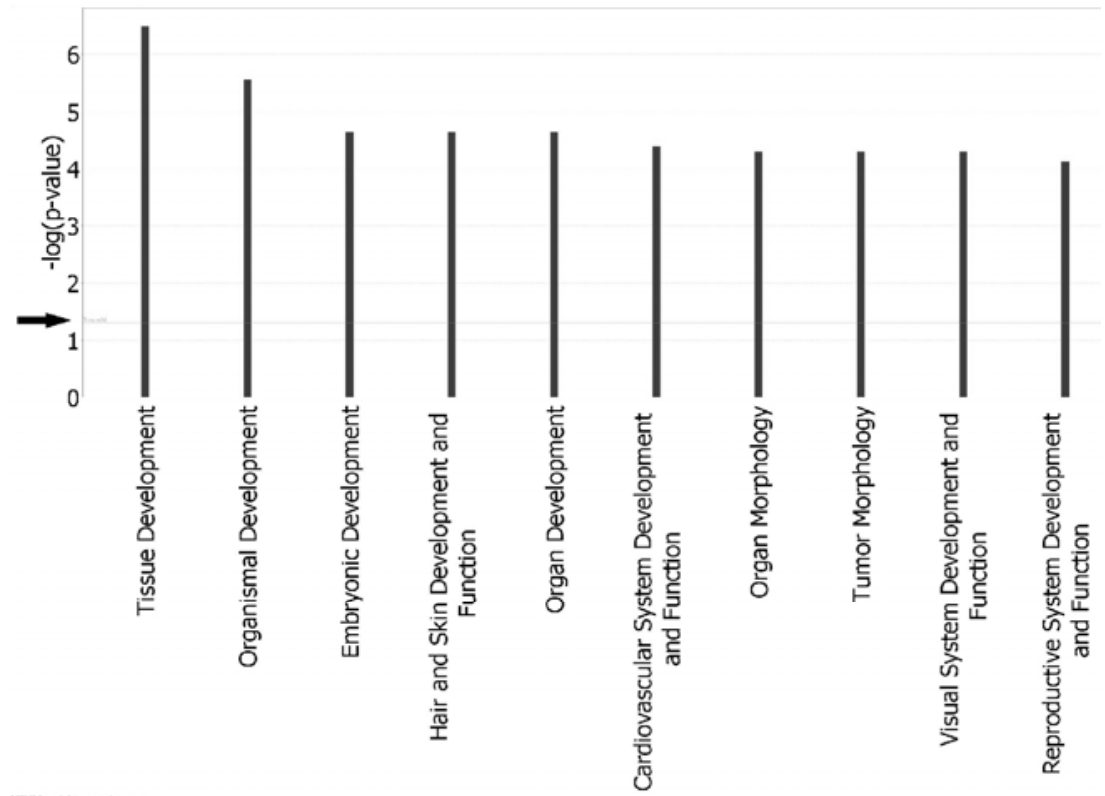

Figure S4. Changes of gene expression in Physiological System Development and Function pathway were analysis the same way as in Fig S3. Top-ten pathways that changed most were listed. Arrow indicates threshold of significance. 
Supplementary Table T1. Forward (F) and Reverse (R) primers used for PCR validation of microarray data. $\mathrm{bp}=$ base pair.

\begin{tabular}{|c|c|c|c|}
\hline Gene Name & Primer Sequence & $\begin{array}{l}\text { Product } \\
\text { size (bp) }\end{array}$ & $\begin{array}{c}\text { Gene Bank } \\
\text { No. }\end{array}$ \\
\hline GAPDH & $\begin{array}{l}\text { F: 5' CGCTCTCTGCTCCTCCTGTT 3' } \\
\text { R: 5'CCATGGTGTCTGAGCGATGT 3' }\end{array}$ & 82 & NM_002046 \\
\hline NFKB1 & $\begin{array}{l}\text { F: 5' ATGTATGTGAAGGCCCATCC 3' } \\
\text { R: 5' TTGCTGGTCCCACATAGTTG 3' }\end{array}$ & 105 & NM_003998 \\
\hline SIPA1L2 & $\begin{array}{l}\text { F: 5' TGGGGAGCGTTCTCCATCACCA 3', } \\
\text { R: 5' CAGGTGCTGCACTTCTGCTTGGA 3, }\end{array}$ & 130 & NM_020808 \\
\hline APOD & $\begin{array}{l}\text { F: 5' CTCCGGTGCAGGAGAATTT 3' } \\
\text { R: 5' CAGCGTCCATTCTCAAAGGT 3, }\end{array}$ & 91 & NM_001647 \\
\hline $\mathrm{CCND} 2$ & $\begin{array}{l}\text { F: 5' AGCGGGAGAAGCTGTCTCTGATCC 3' } \\
\text { R: 5' TGCTCCCACACTTCCAGTTGCG 3' }\end{array}$ & 119 & NM_001759 \\
\hline UBD & $\begin{array}{l}\text { F: 5' TGTCTGCAGAGATGGCTCC 3' } \\
\text { R: 5' TCATATGGGTTGGCATCAAA 3' }\end{array}$ & 94 & NM_006398 \\
\hline DKK3 & $\begin{array}{l}\text { F: 5' TCACATCTGTGGGAGACGAA 3', } \\
\text { R: 5' CAGGTGTACTGGAAGCTGGC 3' }\end{array}$ & 106 & NM_013253 \\
\hline TGFBI & $\begin{array}{l}\text { F: 5' CCAAAGGAAAATCTGTGGCA 3' } \\
\text { R: 5' TTGAGAGTGGTAGGGCTGCT 3' }\end{array}$ & 107 & NM_000358 \\
\hline GREM1 & $\begin{array}{l}\text { F: 5' GTCACACTCAACTGCCCTGA 3', } \\
\text { R: 5' GGTGAGGTGGGTTTCTGGTA 3, }\end{array}$ & 375 & NM_013372 \\
\hline SFRP2 & $\begin{array}{l}\text { F: 5' CGACATAATGGAAACGCTTTG 3, } \\
\text { R: 5' TCTTGCTCTTGGTCTCCAGG } 3{ }^{\prime}\end{array}$ & 110 & NM_003013 \\
\hline COL I & $\begin{array}{l}\text { F: 5' GTCACCCACCGACCAAGAAACC 3, } \\
\text { R: 5' AAGTCCAGGCTGTCCAGGGATG 3, }\end{array}$ & 121 & NM_000088 \\
\hline SPARC & $\begin{array}{l}\text { F: 5' CTTCAGACTGCCCGGAGA 3' } \\
\text { R: 5' GAAAGAAGATCCAGGCCCTC } 3{ }^{\prime}\end{array}$ & 90 & NM_003118 \\
\hline POSTN & $\begin{array}{l}\text { F: 5', TTTGGGCACCAAAAAGAAAT 3', } \\
\text { R: 5' TTCTCATATAACCAGGGCAACA } 3{ }^{\prime}\end{array}$ & 110 & NM_006475 \\
\hline
\end{tabular}

Supplementary Table T2 List of all up-regulated and down-regulated genes with a >2fold change

\begin{tabular}{lcc}
\hline Symbol & Accession & $\begin{array}{c}\text { Fold change } \\
\text { (ASC vs SVF) }\end{array}$ \\
\hline Up-regulated in SVF & NM_006398.2 & -7.711138 \\
UBD & NM_000211.1 & -6.563432 \\
ITGB2 & NM_001647.2 & -6.434554 \\
APOD & NM_004821.1 & -6.031195 \\
HAND1 & NM_005084.2 & -5.715994 \\
PLA2G7 & NM_001289.4 & -5.602651 \\
CLIC2 & NM_006732.1 & -5.366914 \\
FOSB & NM_171999.2 & -5.024266 \\
SALL3 & NM_004093.2 & -4.924205 \\
EFNB2 & &
\end{tabular}




CXCL5
ACRC
GCA
FLJ27255
ITGAX
AGPAT9
IRX6
LPCAT2
ARHGDIB
ANKRD38//IKANK4
EFCAB7
FABP4
FOSL1
RPRM
NCKAP1L
LCP1
CCND2
SIPA1L2
LTB
CD36
CD300LB
TCEAL7
IFRD1
CCNL1
C7Orf53
CFD
THBD
IL32
IRF1
TRIML2
IRF8
CRY1
RTTN
LITAF
FOS
SLC25A33
FAM21A
EGR2
MTHFD2L
FABP3
UNQ6975
LINCR

NM_002994.3

$-4.327044$

NM_052957.3

$-4.261363$

NM_012198.2

$-4.188751$

NM_207501.1

$-3.790181$

NM_000887.3

$-3.743045$

NM_032717.3

$-3.734431$

NM_024335.2

$-3.715168$

NM_017839.3

$-3.693823$

NM_001175.4

$-3.600235$

NM_181712.4

$-3.410388$

NM_032437.1

$-3.322631$

NM_001442.1

$-3.322481$

NM_005438.2

$-3.275004$

NM_019845.2

$-3.194814$

NM_005337.4

$-3.142205$

NM_002298.2

$-3.003938$

NM_001759.2

$-2.894052$

NM_020808.3

$-2.881543$

NM_002341.1

$-2.798827$

NM_001001548.1

$-2.777695$

NM_174892.2

$-2.751698$

NM_152278.2

$-2.739101$

NM_001550.2

$-2.733833$

NM_020307.2

$-2.648373$

NM_182597.1

NM_001928.2

$-2.611317$

NM_000361.2

NM_001012636.1

$-2.559145$

$-2.554253$

NM_002198.1

$-2.486456$

NM_173553.1

$-2.464025$

$-2.446561$

NM_002163.2

$-2.437274$

NM_004075.2

$-2.418118$

NM_173630.2

$-2.39272$

NM_004862.2

$-2.372638$

NM_005252.2

NM_032315.2

$-2.362312$

$-2.360883$

NM_001005751.1

$-2.316277$

NM_000399.2

$-2.299985$

NM_001004346.2

$-2.295357$

NM_004102.3

$-2.286895$

XM_941319.1

NM_001080535.1

$-2.283566$

$-2.26661$ 


\begin{tabular}{|c|c|c|}
\hline C1orf162 & NM_174896.2 & -2.266048 \\
\hline ZIC2 & NM_007129.2 & -2.228865 \\
\hline NFKBIE & NM_004556.2 & -2.21538 \\
\hline NSMAF & NM_003580.2 & -2.208211 \\
\hline ZBTB2 & NM_020861.1 & -2.20302 \\
\hline NID1 & NM_002508.2 & -2.19089 \\
\hline NFKB1 & NM_003998.2 & -2.18274 \\
\hline RPF2 & NM_032194.1 & -2.17338 \\
\hline LOC390557 & XM_001726973.1 & -2.159429 \\
\hline SLC9A3R1 & NM_004252.2 & -2.156407 \\
\hline DNTTIP2 & NM_014597.3 & -2.155031 \\
\hline APOC1 & NM_001645.3 & -2.154229 \\
\hline LTA & NM_000595.2 & -2.144689 \\
\hline ATF3 & NM_001040619.1 & -2.130384 \\
\hline FRG1 & NM_004477.2 & -2.127951 \\
\hline PLA2G4C & NM_003706.1 & -2.111807 \\
\hline RSRC2 & NM_023012.4 & -2.093348 \\
\hline ATP6V1B2 & NM_001693.3 & -2.091477 \\
\hline PIK3CG & NM_002649.2 & -2.08022 \\
\hline CYLD & NM_015247.2 & -2.073035 \\
\hline ANKRD10 & NM_017664.2 & -2.061638 \\
\hline TAF4B & NM_005640.1 & -2.017993 \\
\hline ARID4B & NM_016374.5 & -2.015602 \\
\hline MITD1 & NM_138798.1 & -2.00058 \\
\hline \multicolumn{3}{|c|}{ Down-regulated in SVF } \\
\hline IGFBP7 & NM_001553.1 & 2.019207 \\
\hline PLOD1 & NM 000302.2 & 2.026151 \\
\hline ENTPD4 & NM 004901.2 & 2.031088 \\
\hline DPYSL2 & NM_001386.4 & 2.041851 \\
\hline CD99 & NM_002414.3 & 2.048218 \\
\hline TPM2 & NM_213674.1 & 2.062492 \\
\hline P4HA1 & NM_001017962.1 & 2.06408 \\
\hline PPIC & NM_000943.4 & 2.07825 \\
\hline QSOX1 & NM_001004128.2 & 2.100773 \\
\hline LOC652815 & X⿳亠丷⿵冂_942488.1 & 2.104445 \\
\hline SCRN1 & NM_014766.3 & 2.10685 \\
\hline FNDC1 & NM_032532.1 & 2.112626 \\
\hline HIST2H2AC & NM_003517.2 & 2.125947 \\
\hline TRIB2 & NM_021643.1 & 2.127267 \\
\hline B4GALT1 & NM_001497.2 & 2.16613 \\
\hline FKBP9L & NM_182827.1 & 2.166701 \\
\hline BGN & NM_001711.3 & 2.20144 \\
\hline
\end{tabular}




\begin{tabular}{|c|c|c|}
\hline$X G$ & NM_175569.1 & 2.211911 \\
\hline WDR54 & NM_032118.2 & 2.213135 \\
\hline C1orf54 & NM_024579.2 & 2.246802 \\
\hline IGFBP6 & NM_002178.2 & 2.260575 \\
\hline PLOD2 & NM_182943.2 & 2.292895 \\
\hline FSTL1 & NM_007085.3 & 2.318603 \\
\hline ADAM19 & NM_033274.2 & 2.343654 \\
\hline MXD4 & NM_006454.2 & 2.365293 \\
\hline LAMB2 & NM_002292.3 & 2.387693 \\
\hline FBLN1 & NM_006487.2 & 2.418595 \\
\hline FAM46A & NM_017633.2 & 2.433945 \\
\hline LOC100132535 & XR_038625.1 & 2.439969 \\
\hline F3 & NM_001993.2 & 2.446623 \\
\hline EHD2 & NM_014601.2 & 2.480436 \\
\hline VKORC1 & NM_024006.4 & 2.494336 \\
\hline COL11A1 & NM_080629.2 & 2.50218 \\
\hline SERPINF1 & NM_002615.4 & 2.655044 \\
\hline LOC100129667 & XR_037840.1 & 2.769492 \\
\hline FBLN2 & NM_001998.2 & 2.798432 \\
\hline SH3PXD2A & NM_014631.2 & 2.824625 \\
\hline P4HA2 & NM_001017973.1 & 2.832366 \\
\hline EFEMP2 & NM_016938.2 & 2.833782 \\
\hline RNY3 & NR_004392.1 & 2.948212 \\
\hline LOXL3 & NM_032603.2 & 3.072924 \\
\hline FN1 & NM_212474.1 & 3.253216 \\
\hline SFRP2 & NM_003013.2 & 3.32327 \\
\hline COL5A2 & NM_000393.3 & 3.63136 \\
\hline COL1A2 & NM_000089.3 & 3.631591 \\
\hline SLC16A3 & NM_004207.2 & 3.949788 \\
\hline $\mathrm{CDH} 2$ & NM_001792.2 & 4.082883 \\
\hline COL1A1 & NM_000088.3 & 4.273946 \\
\hline TGFBI & NM_000358.1 & 4.396439 \\
\hline SNORD46 & NR_000024.2 & 4.458385 \\
\hline POSTN & NM_006475.1 & 5.025622 \\
\hline DKK3 & NM_013253.4 & 5.166207 \\
\hline COL5A1 & NM_000093.3 & 5.221677 \\
\hline SPARC & NM_003118.2 & 5.568203 \\
\hline GREM1 & NM_013372.5 & 5.671927 \\
\hline MFGE8 & NM_005928.1 & 5.955669 \\
\hline COL3A1 & NM_000090.3 & 6.279095 \\
\hline LOX & NM_002317.3 & 7.003025 \\
\hline
\end{tabular}




\section{Chapter 7}

\section{General Discussion*}

This chapter is an overall discussion of the entire thesis, with future perspectives. 


\section{General discussion}

Autologous Chondrocyte Implantation (ACI) is one of the most popular treatments for cartilage defects. However, several drawbacks especially the two step procedure and the mandatory in vitro expansion of chondrocytes, increase its costs and prevent it from broader application [1]. Partial replacement of chondrocytes by mesenchymal stem cells (MSCs) can potentially simplify the two step procedure to a single step surgery by excluding the in vitro cell expansion. In vitro studies showed that the amount of cartilage produced in co-culture of MSCs and chondrocytes equals or is even higher than that formed by pure chondrocyte or MSCs [2-5]. Unknown mechanisms in the cross-talk between chondrocytes and MSCs are driving the cartilage formation in the co-cultures to a higher level than in mono-culture. Studying this mechanism may help to realize the idea of one step surgery in clinical application for cartilage repair. Therefore, in this thesis, we tried to uncover the mechanism of cellular interactions between MSCs and chondrocytes in a co-culture or co-implantation system. To achieve this goal, several questions were formulated which need to be answered: 1) which mechanism is responsible for the extracelluar matrix deposition in co-culture pellets of chondrocytes and MSCs; 2) which molecules are involved in these interactions; and 3) how can this knowledge about cellular interactions be translated into clinical practice.

\section{Trophic effects of MSCs in cartilage regeneration}

Hendriks et al., reported that cartilage matrix deposition could be enhanced by co-culturing primary chondrocytes with a variety of cell types, including human expanded chondrocytes, human dermal fibroblasts, mouse embryonic stem cells, mouse-3T3 feeder cells, or human mesenchymal stem cells in cell pellets [6]. Their data showed that co-culture pellets seeded with approximately $20 \%$ of chondrocytes produced comparable amount of GAGs to mono-culture of chondrocytes after a 3 to 4 weeks of culture. Such synergistic effect of matrix deposition in co-cultures of chondrocytes with other cell types was defined as chondro-induction [7]. Two hypotheses have been proposed to explain chondro-induction in co-cultures of MSCs and primary chondrocytes. First, chondrocytes produce factors which stimulate MSCs to actively undergo chondrogenic differentiation, thereby increasing cartilage formation. 
Such factors produced by chondrocytes could be secreted into the surrounding environment or delivered by direct cell-cell contact. Second, MSCs create a better microenvironment for chondrocytes, so that more cartilage matrix is produced by chondrocytes. Limited scientific evidence is available for supporting both hypotheses, predominantly due to the insufficient tracking of the individual role of each cell type in cartilage formation in co-cultures.

Bone marrow give rise to one group of multi-potent progenitors named mesenchymal stromal or stem cells (MSCs) [8]. It has been well demonstrated that these cells are able to differentiate into osteoblasts [9], chondrocytes [10], myoblasts [11], tendon and ligament [12], adipocytes [13], and cells of other connective tissues [14]. Like bone marrow, adipose tissues also give rise to mesenchymal stem cells which can be isolated from the stromal vascular fraction (SVF) [15]. These adipose tissue-derived MSCs are also called adipose stem cells (ASCs). They are capable of forming tissues like fat, bone, cartilage and muscles under specific culture conditions [16]. MSCs could also be isolated from many other adult tissues, such as but not limited to muscle[17], synovium [18], and dental pulp [19]. Traditionally, it was believed that MSCs repair damaged tissue by differentiating into tissue specific cells to replace lost cells [20]. However, recent reports suggests that the beneficial effects of transplanted MSCs in restoring damaged tissue is not completely due to differentiation into tissue specific cells [21-23]. A role of MSCs has been proposed in tissue repair by acting as trophic mediators secreting factors that promote local cells to regenerate the damaged tissue [24-25]. Besides describing the process in which MSCs secrete factors that stimulate nearby cells to release functionally bioactive molecules[26], the term trophic also refers to the effect of MSCs on viability, proliferation, and matrix production of neighboring cells. This effect is also mediated by soluble factors. This concept provides us with a new angle of looking at the function of MSCs in tissue repair. Nowadays the trophic role of the MSC in tissue repair is considered even more important than the traditional thought of MSCs differentiating into tissue-specific cells [27].

In this thesis, our data demonstrated that enhanced cartilage matrix in coculture or co-implantation is due to proliferation and matrix production of chondrocytes stimulated by MSCs' trophic effects, which can be further dissected into 4 distinct 


\section{Chapter 7}

actions: 1) MSCs increase GAG formation and extracellular matrix production of chondrocytes in co-culture pellets by stimulating chondrocyte proliferation; 2) During cartilage formation in co-culture pellets MSCs preferentially die 3) the trophic effects of MSCs in co-culture are general observations that are not influenced by cell culture conditions and cell sources of MSCs; 4) MSCs induce proliferation of chondrocytes in co-culture pellets by secreting FGF-1; 5) Uncultured SVF has a stronger trophic effect than in vitro expanded ASC. These evidences favoring the second hypothesis for explanation of chondro-induction were further supported by independent studies performed by Acharya et al., [28] and Meretoja et al.[29]. Although we cannot completely exclude the possibility that a small group of MSCs differentiated into chondrocytes and directly deposit cartilage matrix, data from others' and our studies suggest that this this effect appears to be minor.

\section{Secreted factors in the interaction between MSCs and chondrocytes}

In this thesis, we also investigated the molecular mechanism underlying the cellular interactions of MSCs and chondrocytes with regards of the trophic effects of MSCs on chondrocytes. We provide evidence to support the hypothesis that FGF-1 is responsible for MSCs induced chondrocyte proliferation in co-culture pellets (Chapter 5). But there are more questions regarding the molecular mechanism of MSC's trophic role which remain to be answered. One of these interesting questions we haven't looked into is the reason why MSCs die in the co-culture pellets. In other studies regarding cell therapies, MSCs were injected into diseased animals, either systemically or directly into tissues of interest [30-31]. One major issue in these studies is low cell viability after cell transplantation, despite the fact that beneficial effects are reported in most of these transplantation studies. Especially, massive cell death of transplanted MSCs is observed when they are injected directly into the tissue of interest [32], where they have direct contacts with cells at sites of injury. Several hypotheses have been proposed to explain the low survival rates of transplanted MSCs. Hypoxia, nutrition limitation and inflammation are all considered as possible reasons for the death of MSCs in vivo. Somehow, these conditions could also exist in our pellet co-culture system. For example, cell aggregation might prevent oxygen and nutrients diffusion into the middle of the pellets. This may explain the presence of apoptotic cells in pellet monocultures of 
MSCs at week 1 and week 2 of culture (Chapter 3). Nutrient or oxygen deprivation is unlikely to explain the increased apoptosis of MSCs in co-culture pellets with chondrocytes, since apoptotic cells are mainly found in the periphery of the cell pellet in close contact with chondrocytes and not in the center. This finding suggests that signals from the chondrocytes are involved in inducing cell death of the MSCs. Chondrocytes isolated from late stages of osteoarthritis may release a number of inflammatory factors which may induce apoptosis of MSCs co-cultured with chondrocytes. This may contribute to the increased apoptotic rate of MSCs in co-culture pellets compared to pellet monocultures of MSCs (Chapter 3 and 6). So, the effects of the up-mentioned cell death inducers in our study obviously are amplified by the presence of chondrocytes. However, increased death of MSC is also observed in co-cultures with healthy bovine primary chondrocytes. This suggests that communication of chondrocytes, either derived from diseased or healthy tissue, with MSCs per se is responsible for the increased cell death. Besides inflammatory factors, it's therefore likely that chondrocytes may produce as yet unknown factors which may actively kill MSCs in order to make space for their extracellular matrix which is gradually expanding. It is well-known that chondrocytes are tolerant to stressful environment, and that environmental stresses are important for their matrix deposition. For example, chondrocytes cultured in hypoxic chamber produced more GAGs [33] and depletion of serum from culture medium also has positive effects on matrix formation of chondrocytes [34]. It is very likely that chondrocytes are able to secrete stress related factors and cytokines into the surrounding environment when they aggregate to form 3dimentional constructs. These factors eventually help matrix deposition of chondrocytes, meanwhile unknown mechanisms prevent these factors from doing harm to chondrocytes.

The death of MSCs is clearly an indication that MSCs are under stress when co-cultured with chondrocytes probably due to some factors produced by chondrocyte as we discussed above. From literature, it is known that FGF-1 can be secreted by cells under stresses like heat shock [35], hypoxia [36], serum starvation [37], and low-density lipoproteins (LDLs) [38]. It was documented that endogenous FGF-1 could function as neurotrophic factors for PC12 cells, a cell line derived from a pheochromocytoma of the 


\section{Chapter 7}

rat adrenal medulla [39]. This neurotrophic effects increase the viability of PC12 cells in low serum cultivation. Results from another study indicated that p53-dependent apoptosis of PC12 cells can be reduced by adding FGF-1 in culture medium [40]. Based on these data from literature, we may hypothesize that MSCs are trying to respond to stresses coming from chondrocytes by up-regulating and secreting FGF-1. FGF-1 may partly protect MSCs from apoptosis but simultaneously also increase proliferation of chondrocytes. Eventually, the stress from chondrocyte is too strong to be protected by FGF-1. Even though FGF-1 expression increases in co-culture, most of the MSCs still died if they did not differentiate into chondrocytes. These MSC derived chondrocytes may have some unknown mechanisms to neutralize the stress in the co-culture environment.

Our data from co-culture models indicated that chondrocytes induce FGF-1 expression/secretion by MSCs via an unknown mechanism (chapter 5). It has been revealed that treatment of chondrocytes with FGF family members may increase the expression of Collagen 1, thus promoting the formation of fibrocartilage [41-42]. Meanwhile, results from xenogenic co-cultures of bovine chondrocytes and human MSCs showed that expression of BMP-2 in chondrocytes was increased by co-culture with MSCs (Chapter 5). It was reported that overexpression of BMP-2 in mesenchymal cells eventually lead to hypertrophy of chondrocytes [43]. From the classical interactive model of epithelium and mesenchyme during vertebrate limb development, we know that FGF signaling from the epithelium induces expression of Sonic Hedgehog ( $\mathrm{SHH})$ in the mesenchyme. As a feedback, SHH maintains the expression of Gremlin1, which antagonizes the activity of BMPs. A balance in BMP and Gremlin1 regulates the activation of FGF signaling [44]. From more recent studies, we noticed that FGF and BMP signaling act in an antagonistic manner regulating chondrocyte proliferation, thus a balance of the two signaling pathways is essential for the transition of chondrocytes from a more differentiated status to a proliferative status [45]. It was also reported that BMP signaling was counterbalanced by FGF-signaling and other MAP kinase activators in many processes of vertebrate development such as neurogenesis and bone formation [46]. This counterbalance of FGF and BMP signaling may lead to compensation of FGF's fibrotic effects and BMP's hypertrophic effects. Interestingly, neither fibrosis nor 
hypertrophy is observed in our co-culture pellets but it is unclear whether these observations are casually related. The crosstalk of MSCs and chondrocytes mediated by FGF1 and BMP2 is summarized in figure 1.

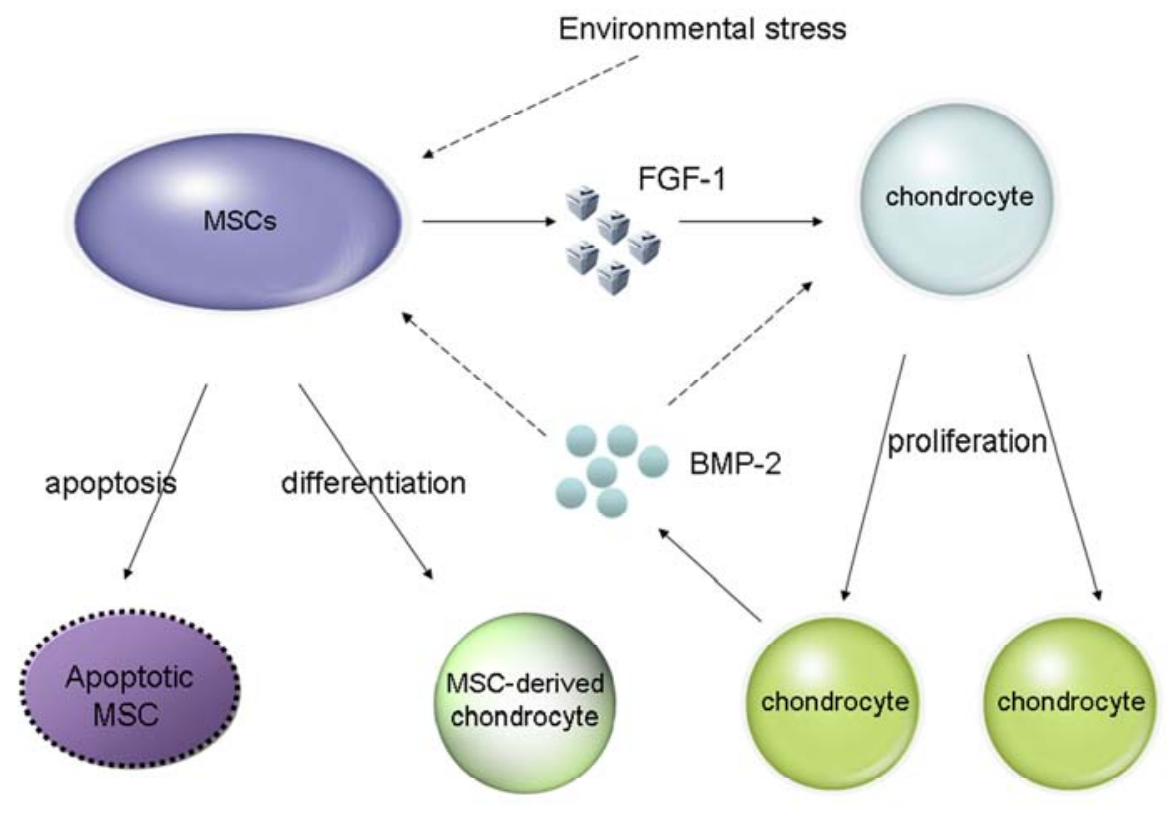

Figure1 Schematics of crosstalking between chondrocytes and MSCs mediated by FGF1 and BMP-2.

The death of MSCs may explain another interesting observation which is why GAG/DNA is increased in co-culture pellets. Irrespective of the source of MSCs, we observed more GAG/DNA in co-culture pellets with chondrocyte as well as more cell death in these pellets. The observation could be explained by a hypothesis that only cells which make cartilage matrix can survive in these co-culture pellets. So, if we find out the underlying mechanism of increased death of MSCs in co-culture pellets with MSCs and chondrocytes, we could explain why more GAG/DNA can be observed in SVF co-cultured with chondrocytes, because cells don't produce GAGs disappear. This hypothesis could be supported by the fact that higher GAG/DNA, more cell death, as well as higher final proportion of chondrocytes are all found in co-culture of SVF and 
chondrocytes when compared to co-culture of ASC and chondrocytes. However, there is still a possibility that MSCs produce a factor(s) that up-regulate chondrogenic genes in chondrocytes and eventually increase matrix formation in co-culture pellets. Alternatively MSCs may facilitate chondrocytes in producing growth factors which stimulate cartilage matrix formation. For example, increased expression of BMP-2 by chondrocytes in co-culture pellets could be the driving force for higher GAG/DNA (Chapter 5).

\section{Applications of trophic effects in translational medicine}

Translational medicine integrates research from fundamental sciences, social sciences and political sciences to optimize patient care that may extend healthcare services for human beings [47]. Its ultimate goal is to turn appropriate biological findings into treatments or medical devices that eventually improve the management of patients. Scientific findings presented in this thesis point out new functions of MSCs in tissue repair in particular during cartilage repair. This knowledge could potentially be translated into stem-cell based therapies.

\section{One-step surgical therapy}

A one-step surgical therapy has been proposed to improve the performance of ACI. In the scheme of One-Step ACI, stem cells are obtained and co-implanted into the patient together with chondrocytes isolated from the non-weight bearing part of the diseased joint in one surgical procedure. In a One-Step procedure the necessity of in vitro cell expansion, which would subject the patients to a second procedure and would cause dedifferentiation of chondrocytes is avoided [48]. To be applicable in a One-Step surgical procedure, stem cells sources should be 1) easily isolated in 2) high yield and these cells should have 3 ) beneficial effects on cartilage formation when co-implanted with chondrocytes. Results in Chapter 6 and other studies indicated that SVF from adipose tissue could be one of the best cells source to fulfill these requirements for onestep therapy. First of all, when compared to other cell sources like the bone marrow mononuclear fraction, SVF is more abundant and easier to be isolated with relatively low morbidity and pain [15]. Secondly, SVF can be easily isolated from the patients in large quantities by a simple liposuction. Thirdly, our data demonstrated that SVF increase cartilage matrix formation of chondrocytes both in vitro and in vivo. Animal 
studies are necessary to provide information about practical operation and safety issues. Interactions of cells and scaffold materials are also important in terms of biosafety and therapeutic efficacy. The studies in this thesis pave the way to the clinical studies of testing the feasibility of one-step therapy using SVF in combination with primary chondrocytes which could eventually lead to new regenerative therapies for cartilage defects.

\section{Osteoarthritis}

Osteoarthritis (OA) is known as a heterogeneous disease leading to gradual degradation of joint tissues. It involves both articular cartilage and subchondral bone [49]. It is considered as the most common joint disease that is highly associated with age. Its treatment includes combinations of exercise, lifestyle modification, and analgesics. Since there is no way to stop its progression, patients eventually undergo total joint replacement surgery [49]. As our understanding about MSCs accumulates, people start thinking of using MSCs instead of chondrocytes to regenerate and maintain the function of articular cartilage in OA. Since MSCs can regulate the immune response and adjust internal microenvironment in joints, MSCs may initiate endogenous repair mechanisms in the OA joint through direct cell- cell interaction or the secretion of various factors [50]. In both animal models and clinical studies, implanted MSCs delayed the progression of OA [51-52]. The underlying mechanism is not clear so far. However, it has been clearly demonstrated that implanted MSCs were integrated into the defect site and contributed to tissue repair. Combined with the knowledge presented in this thesis, we propose the hypothesis that MSCs influence the local environment and activate endogenous precursors or stem cells to exert a beneficial effect on tissue repair in delaying the progression of OA. Generally, such beneficial effects could also be classified as a trophic effect of MSCs. It would be interesting to explore whether there is a role of MSC secreted FGF-1 in the proposed beneficial role of MSCs in osteoarthritis. In this respect it is noteworthy to mention that a family member of FGF-2, FGF-18 is currently explored for treatment of osteoarthritis in clinical trials ([53-54]) Although many experimental and clinical studies are needed before MSCs can be applied in the clinic for OA patients; we believe that findings from this thesis may offer great potential in developing new MSC-based therapeutics relieving degenerative joint diseases. 


\section{References}

1. Kon E, Filardo G, Di Martino A, et al. ACI and MACI. J Knee Surg 2012;25:17-22.

2. Mo XT, Guo SC, Xie HQ, et al. Variations in the ratios of co-cultured mesenchymal stem cells and chondrocytes regulate the expression of cartilaginous and osseous phenotype in alginate constructs. Bone 2009; $45: 42-51$.

3. Tsuchiya K, Chen G, Ushida T, et al. The effect of coculture of chondrocytes with mesenchymal stem cells on their cartilaginous phenotype in vitro. Materials Science \& Engineering CBiomimetic and Supramolecular Systems 2004;24:6.

4. Hildner F, Concaro S, Peterbauer A, et al. Human adipose-derived stem cells contribute to chondrogenesis in coculture with human articular chondrocytes. Tissue Eng Part A 2009;15:3961-3969.

5. Vadala G, Studer RK, Sowa G, et al. Coculture of bone marrow mesenchymal stem cells and nucleus pulposus cells modulate gene expression profile without cell fusion. Spine (Phila Pa 1976) 2008;33:870-876.

6. Hendriks JAA, Miclea RL, Schotel R, et al. Primary chondrocytes enhance cartilage tissue formation upon co-culture with a range of cell types. Soft Matter 2010;6:5080-5088.

7. Acharya C, Adesida A, Zajac P, et al. Enhanced chondrocyte proliferation and mesenchymal stromal cells chondrogenesis in coculture pellets mediate improved cartilage formation. J Cell Physiol 2012;227:88-97.

8. Caplan AI. Mesenchymal stem cells. J Orthop Res 1991;9:641-650.

9. Haynesworth SE, Goshima J, Goldberg VM, et al. Characterization of cells with osteogenic potential from human marrow. Bone 1992;13:81-88.

10. Yoo JU, Barthel TS, Nishimura K, et al. The chondrogenic potential of human bone-marrowderived mesenchymal progenitor cells. J Bone Joint Surg Am 1998;80:1745-1757.

11. Wakitani S, Saito T, Caplan AI. Myogenic cells derived from rat bone marrow mesenchymal stem cells exposed to 5-azacytidine. Muscle Nerve 1995;18:1417-1426.

12. Young RG, Butler DL, Weber W, et al. Use of mesenchymal stem cells in a collagen matrix for Achilles tendon repair. J Orthop Res 1998;16:406-413.

13. Dennis JE, Merriam A, Awadallah A, et al. A quadripotential mesenchymal progenitor cell isolated from the marrow of an adult mouse. J Bone Miner Res 1999;14:700-709.

14. Studeny M, Marini FC, Dembinski JL, et al. Mesenchymal stem cells: potential precursors for tumor stroma and targeted-delivery vehicles for anticancer agents. J Natl Cancer Inst 2004;96:1593-1603.

15. Zuk PA, Zhu M, Mizuno H, et al. Multilineage cells from human adipose tissue: implications for cell-based therapies. Tissue Eng 2001;7:211-228.

16. Zuk PA, Zhu M, Ashjian P, et al. Human adipose tissue is a source of multipotent stem cells. Mol Biol Cell 2002; 13:4279-4295.

17. Usas A, Huard J. Muscle-derived stem cells for tissue engineering and regenerative therapy. Biomaterials 2007;28:5401-5406.

18. Sakaguchi Y, Sekiya I, Yagishita K, et al. Comparison of human stem cells derived from various mesenchymal tissues: superiority of synovium as a cell source. Arthritis Rheum 2005;52:25212529.

19. Cai X, Zhang Y, Yang X, et al. Uniaxial cyclic tensile stretch inhibits osteogenic and odontogenic differentiation of human dental pulp stem cells. J Tissue Eng Regen Med 2011;5:347-353.

20. Bruder SP, Fink DJ, Caplan AI. Mesenchymal stem cells in bone development, bone repair, and skeletal regeneration therapy. J Cell Biochem 1994;56:283-294.

21. da Silva Meirelles L, Caplan AI, Nardi NB. In search of the in vivo identity of mesenchymal stem cells. Stem Cells 2008;26:2287-2299.

22. Dai W, Hale SL, Martin BJ, et al. Allogeneic mesenchymal stem cell transplantation in postinfarcted rat myocardium: short- and long-term effects. Circulation 2005;112:214-223.

23. Noiseux N, Gnecchi M, Lopez-Ilasaca M, et al. Mesenchymal stem cells overexpressing Akt dramatically repair infarcted myocardium and improve cardiac function despite infrequent cellular fusion or differentiation. Mol Ther 2006;14:840-850.

24. Crigler L, Robey RC, Asawachaicharn A, et al. Human mesenchymal stem cell subpopulations express a variety of neuro-regulatory molecules and promote neuronal cell survival and neuritogenesis. Exp Neurol 2006;198:54-64. 
25. Gnecchi M, He H, Liang OD, et al. Paracrine action accounts for marked protection of ischemic heart by Akt-modified mesenchymal stem cells. Nat Med 2005;11:367-368.

26. Caplan AI, Dennis JE. Mesenchymal stem cells as trophic mediators. J Cell Biochem 2006;98:1076-1084.

27. Kassis I, Vaknin-Dembinsky A, Karussis D. Bone marrow mesenchymal stem cells: agents of immunomodulation and neuroprotection. Curr Stem Cell Res Ther 2011;6:63-68.

28. Acharya C, Adesida A, Zajac P, et al. Enhanced chondrocyte proliferation and mesenchymal stromal cells chondrogenesis in coculture pellets mediate improved cartilage formation. J Cell Physiol 2011.

29. Meretoja VV, Dahlin RL, Kasper FK, et al. Enhanced chondrogenesis in co-cultures with articular chondrocytes and mesenchymal stem cells. Biomaterials 2012.

30. Mooney DJ, Vandenburgh H. Cell delivery mechanisms for tissue repair. Cell Stem Cell 2008;2:205-213.

31. Roh JK, Jung KH, Chu K. Adult stem cell transplantation in stroke: its limitations and prospects. Curr Stem Cell Res Ther 2008;3:185-196.

32. Daley GQ, Scadden DT. Prospects for stem cell-based therapy. Cell 2008;132:544-548.

33. Adesida AB, Mulet-Sierra A, Jomha NM. Hypoxia mediated isolation and expansion enhances the chondrogenic capacity of bone marrow mesenchymal stromal cells. Stem Cell Res Ther 2012;3:9.

34. Felka T, Schafer R, De Zwart P, et al. Animal serum-free expansion and differentiation of human mesenchymal stromal cells. Cytotherapy 2010;12:143-153.

35. Jackson A, Friedman S, Zhan X, et al. Heat shock induces the release of fibroblast growth factor 1 from NIH 3T3 cells. Proc Natl Acad Sci U S A 1992;89:10691-10695.

36. Mouta Carreira C, Landriscina M, Bellum S, et al. The comparative release of FGF1 by hypoxia and temperature stress. Growth Factors 2001;18:277-285.

37. Shin JT, Opalenik SR, Wehby JN, et al. Serum-starvation induces the extracellular appearance of FGF-1. Biochim Biophys Acta 1996;1312:27-38.

38. Ananyeva NM, Tjurmin AV, Berliner JA, et al. Oxidized LDL mediates the release of fibroblast growth factor-1. Arterioscler Thromb Vasc Biol 1997; 17:445-453.

39. Renaud F, Desset S, Oliver L, et al. The neurotrophic activity of fibroblast growth factor 1 (FGF1) depends on endogenous FGF1 expression and is independent of the mitogen-activated protein kinase cascade pathway. J Biol Chem 1996;271:2801-2811.

40. Bouleau S, Parvu-Ferecatu I, Rodriguez-Enfedaque A, et al. Fibroblast Growth Factor 1 inhibits p53-dependent apoptosis in PC12 cells. Apoptosis 2007;12:1377-1387.

41. Ellman MB, An HS, Muddasani P, et al. Biological impact of the fibroblast growth factor family on articular cartilage and intervertebral disc homeostasis. Gene 2008;420:82-89.

42. Stewart K, Pabbruwe M, Dickinson S, et al. The effect of growth factor treatment on meniscal chondrocyte proliferation and differentiation on polyglycolic acid scaffolds. Tissue Eng 2007; 13:271-280.

43. Carlberg AL, Pucci B, Rallapalli R, et al. Efficient chondrogenic differentiation of mesenchymal cells in micromass culture by retroviral gene transfer of BMP-2. Differentiation 2001;67:128-138.

44. Laufer E, Nelson CE, Johnson RL, et al. Sonic hedgehog and Fgf-4 act through a signaling cascade and feedback loop to integrate growth and patterning of the developing limb bud. Cell 1994;79:993-1003.

45. Minina E, Kreschel C, Naski MC, et al. Interaction of FGF, Ihh/Pthlh, and BMP signaling integrates chondrocyte proliferation and hypertrophic differentiation. Dev Cell 2002;3:439-449.

46. Sapkota G, Alarcon C, Spagnoli FM, et al. Balancing BMP signaling through integrated inputs into the Smad1 linker. Mol Cell 2007;25:441-454.

47. Terzic A, Waldman SA. Translational medicine: path to personalized and public health. Biomark Med 2010;4:787-790.

48. Helder MN, Knippenberg M, Klein-Nulend J, et al. Stem cells from adipose tissue allow challenging new concepts for regenerative medicine. Tissue Eng 2007;13:1799-1808.

49. van der Kraan PM, van den Berg WB. Chondrocyte hypertrophy and osteoarthritis: role in initiation and progression of cartilage degeneration? Osteoarthritis Cartilage 2012;20:223-232.

50. Qi Y, Feng G, Yan W. Mesenchymal stem cell-based treatment for cartilage defects in osteoarthritis. Mol Biol Rep 2012;39:5683-5689.

51. Sato M, Uchida K, Nakajima H, et al. Direct transplantation of mesenchymal stem cells into the knee joints of Hartley strain guinea pigs with spontaneous osteoarthritis. Arthritis Res Ther 2012;14:R31. 


\section{Chapter 7}

52. Kasemkijwattana C, Hongeng S, Kesprayura S, et al. Autologous bone marrow mesenchymal stem cells implantation for cartilage defects: two cases report. J Med Assoc Thai 2011;94:395-400.

53. Beyer C, Schett G. Pharmacotherapy: concepts of pathogenesis and emerging treatments. Novel targets in bone and cartilage. Best Pract Res Clin Rheumatol 2010;24:489-496.

54. Malemud CJ. Anticytokine therapy for osteoarthritis: evidence to date. Drugs Aging 2010;27:95115 . 


\section{Summary}

Partial replacement of chondrocytes with stem cells in autologous chondrocyte implantation (ACI) has been proposed as an effective strategy to avoid the in vitro expansion phase of chondrocytes. This expansion phase is necessary to obtain sufficient cells for implantation after harvesting of the cartilage biopsy from the diseased joint. The partial replacement of chondrocytes with stem cells has the potential to bring back the current 2 -step surgical procedure to 1-step surgery. Preliminary studies showed that co-culture of primary chondrocytes with various sources of multipotent stem cells increases the relative amount of cartilage matrix formation compared to monocultures of chondrocytes. The molecular mechanism of this chondro-inductive effect is not known. The aim of this thesis is to provide answers to this question.

In chapter 3 we used human mesenchymal stem cells (hMSCs) in co-culture with human primary chondrocytes (hPCs) or bovine primary chondrocytes (bPCs) and studied the destination and the matrix formation of the individual cell populations in a pellet co-culture system. Enhanced cartilage matrix formation was confirmed by histology and a total glycosaminoglycan (GAG) assay. Species specific quantitative PCR (qPCR) demonstrated that chondrogenic genes were mainly expressed by chondrocytes. Short tandem repeat (STR) analysis combined with species specific qPCR analysis of genomic DNA showed an increase in the ratio of chondrocytes/hMSCs compared to the initial seeding ratio over time. Chondrocyte proliferation in particular, but not hMSC proliferation was increased in co-culture pellets. This effect was partly mimicked by conditioned medium of MSCs culture in 2D. Based on the data from Chapter 3 we concluded that MSCs disappear over time in pellet co-cultures of MSCs and PCs and that increased cartilage formation in these co-cultures pellets was mainly due to a trophic role of the MSCs. In these co-culture pellets MSCs preferentially stimulate chondrocyte proliferation and matrix deposition rather than actively undergoing chondrogenic differentiation themselves.

Since the studies in Chapter 3 were performed in culture medium that was not chondrogenic inductive, in chapter 4 we next tested whether the trophic role of the MSCs was dependent on culturing co-culture pellets in medium compatible with 
chondrogenic differentiation of MSCs. Human MSCs derived from bone marrow and bPCs were co-cultured in chondrogenic medium. Enhanced matrix production was confirmed by GAG quantification. Species specific qPCR demonstrated that cartilage matrix was mainly derived from bovine origin, indicating that very few MSCs underwent chondrogenic differentiation. In addition we showed that co-culture pellets were overgrown by bovine cells over time. To test the influence of cell sources on this trophic effect, MSCs isolated from two other sources, adipose tissue and synovial membrane, were co-cultured with hPCs and their effect on chondrogenic differentiation was compared with hMSCs from bone marrow. GAG assay again confirmed increased cartilage matrix production in co-culture pellets irrespective of the source of the MSCs. Proliferation studies revealed increased chondrocytes proliferation in each condition. STR analysis of genomic DNA showed a decrease in MSCs in all co-culture pellets. Results from this chapter supported our conclusion that the trophic effects of MSCs that stimulated cartilage formation in co-culture pellets were independent of culture conditions or the origin of the MSCs.

In Chapter 3 we showed that MSCs in co-culture with PC secreted soluble factors that increased chondrocyte proliferation. In Chapter 5, we set up experiments to identify these soluble factors. Human MSCs and PCs were co-cultured in chondrocyte proliferation medium and then microarray experiments were performed to identify differentially expressed genes between co-cultures and mono-cultures. Several genes were sorted out as candidate genes for these soluble factors, and validated by qPCR. Of these, Fibroblast growth factor-1 (FGF-1) was considered as one of the most promising candidates to be responsible for chondrocyte proliferation in co-culture pellets. Immuno-fluorescent staining and ELISA were performed to confirm the expression and secretion of FGF-1. These experiments confirmed increased expression of FGF-1 in the co-cultures predominantly in the MSCs. Small chemical inhibitors of the FGF-signaling pathway or specific inhibitors of the FGF receptors decreased chondrocyte proliferation in co-culture. ELISA detected a considerable level of secreted FGF-1 in the conditioned medium of MSCs (CM), which was increased in co-cultures. When FGF-1 activity was neutralized in CM by anti-FGF-1 antibodies, the inductive effect of CM on chondrocyte proliferation was completely blocked, indicating that FGF-1 is the active component in 
CM. Based on the data from Chapter 5 we concluded that MSCs increase FGF-1 secretion in response to co-culture with chondrocytes, which in turn respond by increasing their proliferation.

With the hope of finding a more easily accessible cell source to replace bone marrow MSCs in cartilage repair, we studied the trophic effects of stromal vascular fraction cells (SVF) of adipose tissue in chapter 6. SVF or expanded ASCs, respectively were co-cultured with chondrocytes to evaluate their trophic effect on cartilage formation. Surface marker profiles were determined by FACs, and gene expression profiles were compared by microarray experiments. Then both cell sources were mixed with chondrocytes in a 4:1 ration and incorporated into an alginate gel. These constructs were subsequently implanted into nude mice subcutaneously. GAG quantification indicated that co-culture pellets of SVF and chondrocytes had more GAG deposition than pellets of ASC and chondrocytes. The increase of the chondrocytes proportion in the co-culture pellets was also more pronounced in the SVF co-culture group than in the ASC co-culture group. Data from FACs and microarray indicated that SVF and ASC had different characteristics in cell surface markers and gene expression profile. Based on the results from chapter 6 , we concluded that SVF is possibly a better cell source for regenerating cartilage tissue than cultured ASCs.

In the general discussion (chapter 8), the main conclusions of this thesis are discussed. A few unsolved questions were brought up and new research directions were also pointed out. Regarding the applications in translational medicine, the findings presented in this thesis could possibly contribute to the development of new therapeutic strategies for osteoarthritis and other joint diseases.

Taken together, the core value of this thesis is the identification of mesenchymal stem cells as trophic mediators in cartilage regeneration. Based on our data, we concluded that mesenchymal stem cells either isolated from bone marrow or adipose tissue or synovium can help in regenerating cartilage by increasing chondrocyte proliferation and matrix formation. Increased proliferation is mediated by MSCssecreted factors, one of which is FGF-1. Freshly isolated MSCs isolated out of the stromal vascular fraction of adipose tissue are more trophic than in vitro expanded MSCs. This thesis provides us with a new angle of looking at the functions of MSCs in 
tissue repair prossesses, and potentially leads to the development of a new paradigm on tissue repair especially for new therapeutics of cartilage defects. 


\section{Samenvatting}

Gedeeltelijke vervanging van de chondrocyte populatie met stam cellen in autologe chondrocyte implantatie (ACI) wordt beschouwd als een effectieve strategie voor het vermijden of verkorten van de in vitro expansie fase. Normaliter is een eerste operatie nodig om een zo klein mogelijk biopsie te verkrijgen waaruit chondrocyten worden geïsoleerd. Deze cellen worden dan geëxpandeerd en gedurende een tweede operatie terug ingebracht om het aangedane gewricht te repareren. De gedeeltelijke vervanging van chondrocyten door stamcellen heeft dus het potentieel om het aantal benodigde operaties te reduceren. Voorlopige studies hebben angetoond dat een cokweek van primaire chondrocyten met verschillende typen stamcellen de relatieve hoeveelheid aangemaakte kraakbeen matrix vergroot ten opzichte van een monokweek met daarin alleen primaire chondrocyten. Het hiervoor verantwoordelijke moleculaire mechanisme is tot op heden nog niet bekend. Het doel van deze thesis is het ontrafelen van dit mechanisme.

In hoofdstuk 3 hebben we humane mesenchymale stamcellen (hMSCs) gekweekt in de aanwezigheid van humane primaire chondrocyten (hPCs) of bovine primaire chondrocyten (bPCs). Vervolgens hebben we het lot en de hoeveelheid matrix formatie van elke individuele cel populatie bepaald. Verhoogde mate van kraakbeen formatie was bevestigd met histologie en een kwantitatieve glycosaminoglycaan detectie test. Species specifieke kwantitatieve polymerase kettingreactie (qPCR) toonde aan dat de verhoging van expressie van kraakbeen matrix genen vooral plaats vond in de chondrocyten. Korte tandem herhaling (STR) analyse in combinatie met species specifieke qPCR analyse van genomisch DNA toonde een verhoogde ratio van chondrocyten/hMSCs aan in vergelijking met de initiële verhouding in het begin van de kweek. We konden dit verklaren doordat de chondrocyten, maar niet de hMSCs, meer celdelingen ondergingen in de co-kweken. Dit effect kon gedeeltelijk worden nagebootst door het blootstellen van chondrocyten aan kweekmedium waar alvorens hMSCs in waren gekweekt. Gebaseerd op de data van hoofdstuk 3 konden we concluderen dat over tijd de hMSCs verdwenen uit de originele co-kweek van hMSCs en PCs en dat de verhoogde kraakbeen formatie in deze co-kweken voornamelijk 
veroorzaakt worden door de 'trofische' rol van de hMSCs. Samengevat, de hMSCs stimuleerde celdeling en kraakbeen matrix depositie van chondrocyten zonder in grote mate zelf te differentiëren in kraakbeen makende cellen.

Aangezien de experimenten in hoofdstuk 3 werden uitgevoerd in kweekmedium dat niet chondrogene differentiatie stimuleert hebben we in hoofdstuk 4 getest of de trofische rol van de MSCs afhankelijk was van de chondrogene stimulatie, of het gebrek daarvan, vanuit het kweekmedium. Om dit te testen hebben we hMSCs afkomstig uit het beenmerg en bPCs een co-kweek laten ondergaan in chondrogeen kweekmedium. Verhoogde mate van kraakbeen matrix aanmaak was bevestigd met een kwantitatieve glycosaminoglycaan detectie test. Species specifieke qPCR toonde aan dat de kraakbeen matrix voornamelijk afkomstig was van een bovine afkomst. Dit wekte de suggestie dat erg weinig MSCs chondrogene differentiatie ondergingen. Daarnaast konden we aantonen dat de co-kweken over tijd werden overwoekerd door bovine cellen. Om te testen wat de invloed van de afkomst van de MSCs was op dit trofische effect hebben we ook MSCs werden geïsoleerd vanuit vetweefsel en het synoviale membraan. Deze cellen werden in co-kweek gebracht met hPCs en getest op hun effect op chondrogene differentiatie ten opzichte van het effect van MSCs afkomstig uit het beenmerg. Kwantitatieve glycosaminoglycaan detectie bevestigde verhoogde kraakbeen formatie in alle co-kweken onafhankelijk van de afkomst van de MSCs. Celdeling studies onthulde verhoogde chondrocyte celdeling in elke conditie. STR analyse van het genomische DNA toonde aan dat het relatieve aantal MSCs sterk was verlaagd in alle co-kweken. Samengevat, dit hoofdstuk bewijst dat de trofische effecten van de MSCs op verhoogde mate van kraakbeen formatie in co-kweken met chondrocyten onafhankelijk is van de kweekconditie en de afkomst van de MSCs.

In hoofdstuk 3 toonde we aan dat MSCs, in co-kweken met PCs, factoren uitscheiden die de celdeling van chondrocyte verhoogde. In hoofdstuk 5 hebben we experimenten ontworpen en uitgevoerd om deze factoren te identificeren. hMSCs and PCs werden zowel apart als samen gekweekt en de expressie van alle genen van alle conditie werd getest met behulp van een microarray analyse. Enkele genen werden geselecteerd als kandidaat genen voor de gezochte uitgescheiden factoren en gevalideerd gebruikende qPCR. Van alle kandidaten werd Fibroblast groei factor 1 
(FGF-1) beschouwd als een van de meest belovende kandidaten die verantwoordelijk kon zijn voor de chondrocyte proliferatie in de co-kweken. Immunofluorescentie kleuringen en ELISA testen werden uitgevoerd om expressie en secretie van FGF-1 te valideren op het eiwit niveau. Deze experimenten bevestigden de expressie of FGF-1 in de co-kweken en dat het voornamelijk afkomstig was van de MSCs. Kleine chemische remmers van de FGF-signalerings route of specifieke remmers van de FGF receptoren verlaagde de chondrocyte celdeling in co-kweken. ELISA testen toonde detecteerbare hoeveelheden van FGF-1 in kweekmedium waar MSCs in gekweekt waren en veel hoge concentraties van FGF -1 in kweekmedium waar MSCs en chondrocyten co-kweken waren uitgevoerd. Wanneer in de co-kweken FGF-1 werd geneutraliseerd door middel van anti-FGF-1 antilichamen werd de versnelde celdeling van de chondrocyte volledig geblokkeerd. In het kort, in hoofdstuk 5 laten we zien dat het co-kweken van MSCs met chondrocytes de FGF-1 productie van MSCs verhoogd wat leidt tot versnelde celdeling van chondrocyten.

In het licht van het vinden van cel soorten die gemakkelijker te verkrijgen zijn, dan MSCs uit het beenmerg, voor het repareren van kraakbeen hebben we de trofische effecten van de stromale vasculaire fractie cellen (SVF) van het vetweefsel onderzocht in hoofdstuk 6. SVF of geëxpandeerde vetweefsel stamcellen (ASCs) werden respectievelijk in co-kweek gebracht met chondrocyten om hun trofische effecten om de aanmaak van kraakbeen te evalueren. Oppervlakte marker profielen werden bepaald met behulp van FACS en gene expressie profielen werden bepaald met behulp van microarray experimenten. Beide cel soorten werden gemengd met chondrocyten in een 4:1 verhouding en ingebracht in een alginaat hydrogel. Deze constructen werden vervolgens onderhuids geïmplanteerd in naakte muizen. Glycosaminoglycaan kwantificatie suggereerde dat co-kweken van SVF met chondrocyten meer kraakbeen matrix produceerde dan co-kweken van ASCs met chondrocyten. De relatieve hoeveelheid chondrocyten nam ook sneller toe in co-kweken met SVF dan ASCs. FACS en microarray analyse gaven aan dat SVF en ASCs verschillende oppervlakte marker profielen en gene expressie profielen bezaten. Gebaseerd op de resultaten van hoofdstuk 6 concluderen we dat SVF wellicht een betere cel soort is voor het repareren van kraakbeen weefsel dan gekweekte ASCs. 
In de hoofdstuk 8 wordt een algemene discussie gegeven waarin de belangrijkste conclusies van dit proefschrift worden besproken en onbeantwoorde onderzoeksvragen en onderzoeksmogelijkheden verder worden toegelicht. Aangaande de toepassingen in translationele geneeskunde, de bevindingen van dit proefschrift kunnen bijdragen aan de ontwikkeling van nieuwe therapeutische strategieën voor het behandelen van artrose en andere gewrichtsaandoeningen.

Samengevat, de belangrijkste vooruitgang die dit proefschrift biedt is de identificatie van mesenchymale stamcellen als trofische mediatoren voor kraak regeneratie. Gebaseerd op onze data, concluderen we dat mesenchymale stamcellen geïsoleerd uit beenmerg, vetweefsel of synovium kunnen bijdragen aan het regenereren van gewrichtskraakbeen door de celdeling en kraakbeen matrix aanmaak van chondrocyten te bevorderen. Deze versnelde celdeling wordt bewerkstelligd door factoren die worden uitgescheiden door de stamcellen, waarvan FGF-1 in ieder geval een belangrijk rol speelt. MSCs die vers geïsoleerd zijn uit de stromale vasculaire fractie van het vetweefsel vervullen deze trofische rol in hogere mate dan MSCs die in vitro zijn opgekweekt. Dit proefschrift voorziet ons met een nieuw licht met betrekking to de functies van MSCs in weefsel reparatie en heeft de potentie om te leiden tot een nieuw paradigma van weefsel reparatie, waarvan de ontwikkeling van deze mogelijkheid voor de reparatie van kraakbeen defecten in dit proefschrift centraal stond. 


\section{Acknowledgements}

After four years hard work, I finally get this beautiful Thesis and get the opportunity to thank all the people who helped me during the past years. My first thank goes to dear Professor Marcel Karperien. Thank you for giving me the opportunity to work in your lab. I understand it's a big risk to take a student from a Chinese University where you probably don't know anybody, not to mention he has no master's degree. I hope I didn't let you down with my four years productive research. Thank you for your courage and inspiration on my project. More discussion I did with you, more confident and hopeful I feel. After all these trainings not only on experimental skills but also on paper writing and scientific thinking, I grow up and become a professional scientist. Also thank you for your recommendation to Professor Kandel and Dr. Christopher Chen. Even though I choose UCLA because of its fame and LA's nice weather, I really appreciate your efforts. Let's have more collaboration in cartilage engineering between Enschede and Chengdu, as well as between Enschede and Los Angeles! I would like to thank Professor Clemens van Blitterswijk. Thank you for creating such a wonderful atmosphere of scientific research in the TR department. I'm also grateful to Dr. Jan de Boer for the discussions on my research project and guidance on career development. I would thank Janine for correcting all the errors and typos of my papers and other suggestions about paper writing. You are such a kind person, always ready to help. Many thanks to Lorenzo, for providing me scaffolds of cartilage repair. Thank you Aart and Pamela for helpful discussion during our group meetings.

Then, my thanks go the people in TR and DBE. Jacqueline, thank you for showing me around the campus and Enschede when I first came. Thanks for your technical supports in the labs. I would say you are the first person I come cross once I had problems in the labs. Thank you Anouk, you are like the nanny in TC lab. We missed you a lot during your maternity leave. My roommate and friend, Jeroen, thank you for your jokes making our life cheerful. We met with some misunderstandings, but we finally understand each other's commitments and ambitions in scientific research as time goes on. I still remember the times when I talk about science in our office. Thank 
you, Liliana, for your help with the animal experiments. Bjorn, thank your for your help with the microscope. I admire your expertise in microscopy things. Giulia and Karolina, thank you beautiful ladies for being around, making our office colorful! Thanks to Joyce, our captain of the TR football team. I miss so much the time we play in the Twente league. Hugo, my colleague and teammate, you are the Christiano Ronaldo of our team! Nicolas, you are excellent player not only in scientific research but also in football. Also many thanks to your driving during my first relocation in Enschede. Gustavo, you are the best keeper we have. Also thank you for having me in your wonderful research project. Ellie, Emilie and Nicole, thank you girls for sharing ideas of science. Tim and Parthiban, thank you guys for discussion in the labs and group meetings. Febriyani, Mijke, Janneke, Bach, Anne, Wim, Ana, Chris, Anand, Hement, Anidita, thank you all for being around, making the department cheerful and active! Dank U well! Thank you Henk-Jan for your corporation in the SVF/ASC project. Let's wait for the last experiment!

The following thanks give to my Chinese colleagues and friends. 马斌, 感谢 四年来有你做伴! 我们相差半年入学, 一起做实验, 一起健身, 一起玩儿牌, 一 起答辩, 一起度过了四年美好的时光! 靖微, 忠华, 感谢你们在关键时候给我和 小琼提供了住宿, 也感谢你们做的好吃的! 靖微做的大盘鸡, 太厉害了 还有忠 华的俘虏土豆, 太牛了 刘珺, 李楠, 谢谢你们的招待, 包子做的实在是太好吃 了, 也怀念一起玩儿三国杀的日子; 刘珺, 感谢你在我生病的时候到医院看望, 还有关键时刻解囊相助。佳平大哥, 你是我们的生活百事通啊, 谢谢你的给的建 议! 惠品大哥, 你是我认识的第一个百万富翁, 呵呵, 向你学习! 也感谢你, 在 我和小琼离开的时候开车送我们。洪林, 感谢你给我们带来的愉快时光, 希望像 你一样的学生越来越多, TR 的中国人队伍越来越壮大! 杨亮, 谢谢你教我健身 的方法。然后是国内的老师同学! 我的好兄弟, 好同事, 林云锋, 谢谢你多年来 的帮助和支持, 总是有这样那样的问题给你添麻烦, 谢谢啦! 你的临床背景和领 导能力加上我的科研训练, 会是很好的组合, 希望有机会和你共创一番事业! 包 崇云老师, 谢谢你在我初到荷兰时给予的帮助, 也谢谢每次回国你的款待。感谢 
室友, 哥们儿, 杨浩, 李世林在国内给予的帮助和招待! 感谢华西口腔的刘否, 汤炜，龙杰，苟伟等老师、师兄在学习科研的过程中的指导帮助！

My last thanks give to my family. 感谢父母多年来的养育之恩！仅有高中和 初中学历的你们, 培养出了获得博士学位的儿子, 我的表现没有让你们失望。我 的记忆出奇的好, 三十年来的成长过程, 历历在目。爸爸教会了我做事要能吃 苦, 妈妈教会了我凡事要动脑筋。离家十载, 求学在外, 正是牢记父母的教诲, 让我一次又一次度过难关, 勇敢前进! 我最后的致谢留给心爱的妻子, 小琼。从 相识到相恋, 我们用了三年时间; 从恋爱到结婚, 我们携手走过了五年时光; 从 结婚到我们有了自己的宝贝，我们又一起度过了五年的岁月。生活因你有了色 彩，事业因你有了方向。人生就像海上行船，有起有落，每当我走入低谷的时 候, 总是有你在身边陪伴, 让我收拾心情, 勇敢再战!

So many people helped me during the past few years, making this thesis possibly been published. Sincerely, thank you all!! 


\section{Curriculum Vitea}

Ling Wu was born on $16^{\text {th }}$ April 1982, in Sichuan China. He graduated from Sichuan University with a Bachelor of Science in Biology. In 2005, he started his career in scientific research in West China College of Stomatology of Sichuan Univeristy by pursuing a Master of Science in Oral Surgery. After two years study as master student, he was transferred to a PhD program in the same university. Then in 2008, he aborted his study in China, and moved to department of tissue engineering, University of Twente in the Netherlands to continue his research on cartilage tissue engineering under the supervision of Prof. Marcel Karperien and Prof. Clemens van Blitterswijk. He is currently working as postdoctoral researcher at the department of Orthopaedic Surgery, University of California, Los Angeles, USA. 


\section{List of publications}

\section{Publications related to this thesis:}

Wu L, Prins HJ, Helder M, van Blitterswijk C, Karperien M. 2012 Trophic effects of mesenchymal stem cells in chondrocyte co-cultures are independent of culture conditions and cell sources. Tissue Eng Part A. [Epub ahead of print]

Wu L., Leijten J.C., Georgi N., Post J.N., van Blitterswijk C. and Karperien M. 2011 Trophic effects of mesenchymal stem cells increase chondrocyte proliferation and matrix formation Tissue Eng Part A 17 1425-36

Wu L., Cai X., Lin Y., Karperien M. Regeneration of articular cartilage by adipose tissue derived mesenchymal stem cells: perspectives from stem cell biology and molecular medicine. Manuscript submitted.

Wu L., Leijten J.C., van Blitterswijk C. and Karperien M. Fibroblast Growth Factor -1 is a mesenchymal stem cell secreted factor stimulating chondrocyte proliferation in coculture. Manuscript in preparation.

Wu L, Prins HJ, Helder M, van Blitterswijk C, Karperien M. Better cartilage formation in chondrocyte co-cultures with the stromal vascular fraction of adipose tissue than with adipose stem cells. Manuscript in preparation.

Wu L., Zhou D., Karperien M., Dijkstra P., Feijen J. In Situ Gelating Enzymatically Cross-linked gelatin Hydrogels for Cartilage Tissue Regeneration. Manuscript in preparation.

Wu L., Karperien M. Trophic effects of Mesenchymal stem cells in cartilage repair and its potential application in translational medicine. Manuscript in preparation.

Leijten J, Georgi N, Wu L, van Blitterswijk C, Karperien M. Cell sources for articular cartilage repair strategies: shifting from mono-cultures to co-cultures. Tissue Eng Part B Rev. 2012 Jul 30. [Epub ahead of print]

\section{Selected publications on peer reviewed journals:}

Wu L, Cai X, Ge Y, Wang J, Lin Y. 2012 Secreted factors from fat tissue increase adipogenic differentiation of mesenchymal stem cells. Cell Prol. 45 311-9

Wu L., Cai X., Dong H., Jing W., Huang Y., Yang X., Wu Y. and Lin Y. 2010 Serum regulates adipogenesis of mesenchymal stem cells via MEK/ERK-dependent PPARgamma expression and phosphorylation J Cell Mol Med 14 922-32 
Li X., Yao J., Wu L., Jing W., Tang W., Lin Y., Tian W. and Liu L. 2010 Osteogenic induction of adipose-derived stromal cells: not a requirement for bone formation in vivo Artif Organs 34 46-54

Sun L.Y., Wu L., Bao C.Y., Fu C.H., Wang X.L., Yao J.F., Zhang X.D. and van Blitterswijk C.A. 2009 Gene expressions of Collagen type I, ALP and BMP-4 in osteoinductive BCP implants show similar pattern to that of natural healing bones Materials Science \& Engineering C-Materials for Biological Applications 29 1829-1834

$\underline{\text { Wu L}}$., et al. 2008 Dentin sialophosphoprotein-promoted mineralization and expression of odontogenic genes in adipose-derived stromal cells. Cells Tissues Organs 187 10312

Lin Y.F., Jing W., Wu L., Li X.Y., Wu Y., Liu L., Tang W., Long J., Tian W.D. and Mo X.M. 2008 Identification of osteo-adipo progenitor cells in fat tissue Cell Prolif 41 803-12

Lin Y., Tang W., Wu L., Jing W., Li X., Wu Y., Liu L., Long J. and Tian W. 2008 Bone regeneration by BMP-2 enhanced adipose stem cells loading on alginate gel Histochem Cell Biol 129 203-10

Jing W., Wu L., Lin Y., Liu L., Tang W. and Tian W. 2008 Odontogenic differentiation of adipose-derived stem cells for tooth regeneration: necessity, possibility, and strategy Med Hypotheses 70 540-2

Wu Y, Xiao J, Wu L, Tian W, Liu L. 2008 Expression of glutamyl aminopeptidase by osteogenic induction in rat bone marrow stromal cells. Cell Biol Int. 32 748-53.

Wu L., Wu Y., Lin Y., Jing W., Nie X., Qiao J., Liu L., Tang W. and Tian W. 2007 Osteogenic differentiation of adipose derived stem cells promoted by overexpression of osterix Mol Cell Biochem 301 83-92

Lin Y., Wang T., Wu L., Jing W., Chen X., Li Z., Liu L., Tang W., Zheng X. and Tian W. 2007 Ectopic and in situ bone formation of adipose tissue-derived stromal cells in biphasic calcium phosphate nanocomposite J Biomed Mater Res A 81 900-10

Jing W., Lin Y., Wu L., Li X., Nie X., Liu L., Tang W., Zheng X. and Tian W. 2007 Ectopic adipogenesis of preconditioned adipose-derived stromal cells in an alginate system Cell Tissue Res 330 567-72 